\title{
On metrics and financing for the Sustainable Development Goals
}

Guido Schmidt-Traub

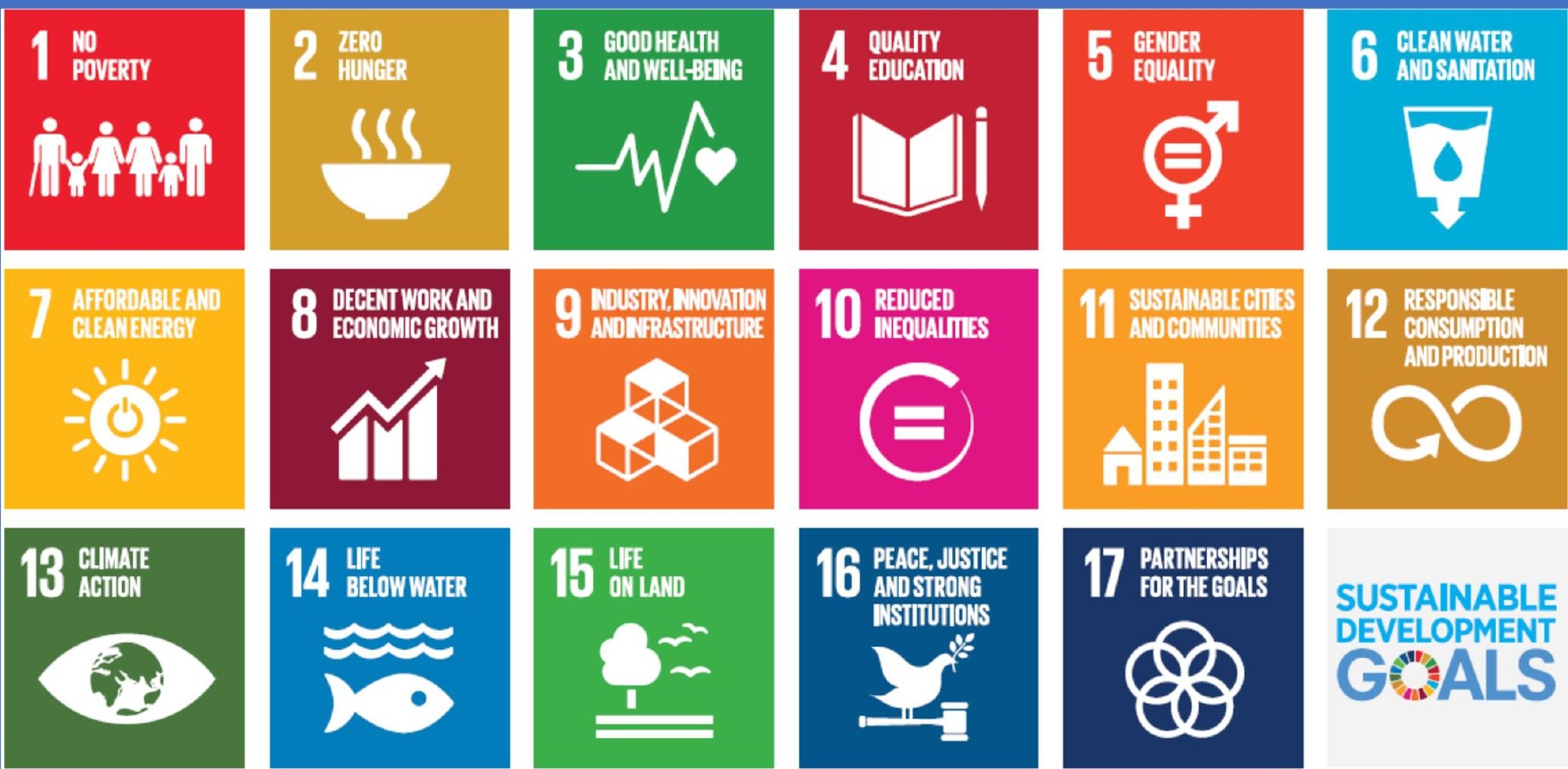





\section{On metrics and financing for the Sustainable Development Goals}

Guido Schmidt-Traub 


\section{Thesis committee}

\section{Promotors}

Prof. Dr E.C. van lerland

Professor of Environmental Economics and Natural Resources

Wageningen University \& Research

Prof. Dr P. Kabat

Director/CEO at the International Institute of Applied Systems Analysis

Laxenburg, Austria

Professor of Earth System Science

Wageningen University \& Research

\section{Co-promotors}

Prof. Dr J.D. Sachs

University Professor

Columbia University

New York, United States of America

Dr J.G. Klomp

Associate Professor, Environmental Economics and Natural Resources Group Wageningen University \& Research

\section{Other members}

Prof. Dr A. Haines, London School of Hygiene and Tropical Medicine, United Kingdom

Prof. Dr W. Lutz, International Institute of Applied Systems Analysis and Vienna University, Austria

Prof. Dr M.W. Hofkes, VU Amsterdam

Prof. Dr R. Leemans, Wageningen University \& Research

This research was conducted under the auspices of the Research School for Socio-Economic and Natural Sciences of the Environment (SENSE). 


\section{On metrics and financing for the Sustainable Development Goals}

\section{Guido Schmidt-Traub}

Thesis

submitted in fulfilment of the requirements for the degree of doctor at Wageningen University

by the authority of the Rector Magnificus,

Prof. Dr A.P.J. Mol,

in the presence of the Thesis Committee appointed by the Academic Board

to be defended in public

on Wednesday, 17 January 2018

at $4 \mathrm{pm}$ in the Aula. 
Guido Schmidt-Traub

On metrics and financing for the Sustainable Development Goals, 238 pages.

PhD thesis, Wageningen University, Wageningen, the Netherlands (2018) With references, with summary in English

ISBN: 978-94-6343-724-0

DOI: $10.18174 / 426260$ 
To LAGS 



\section{Table of contents}

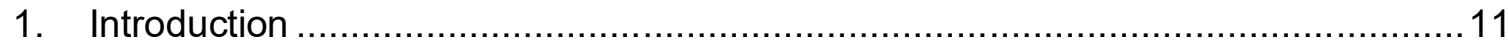

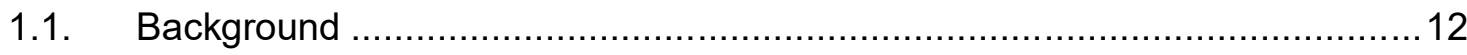

1.2. Problem definition and research questions ............................................... 14

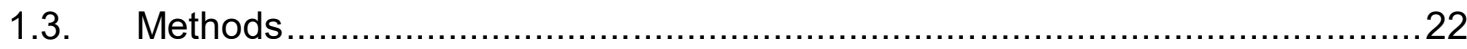

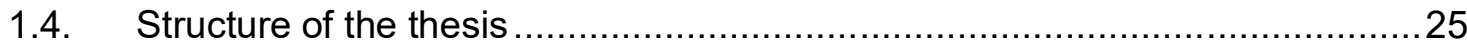

2. National baselines for the Sustainable Development Goals assessed in the SDG

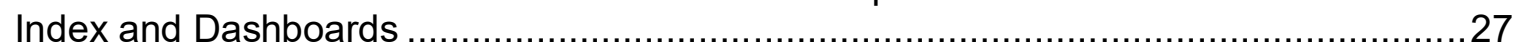

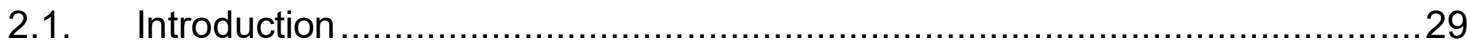

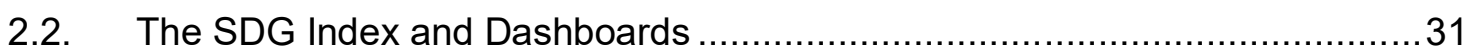

2.3. Interpreting and applying the SDG Index and Dashboards............................. 34

2.4. Major data gaps for the SDGs............................................................. 40

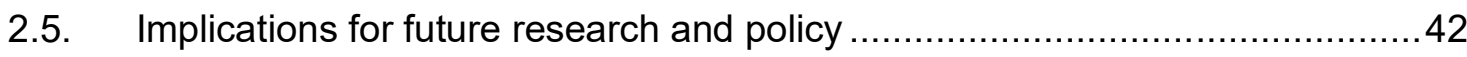

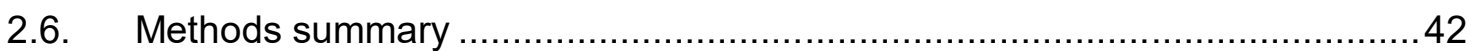

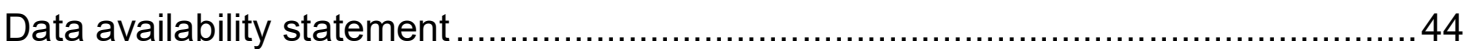

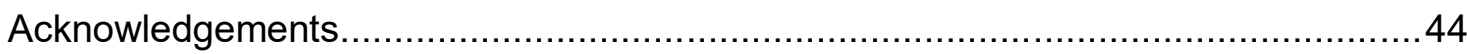

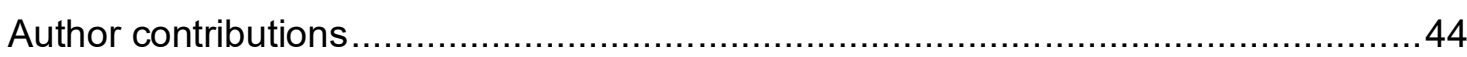

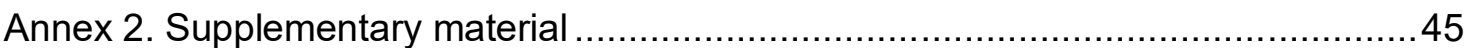

3. From adaptation to climate-resilient development: The costs of climate-proofing the Millennium Development Goals in Africa ................................................................ 77

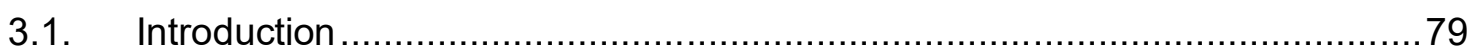

3.2. Why adaptation and development must be integrated ............................... 80

3.3. An analytical approach to integrating adaptation and development ................82

3.4. Estimating the cost of climate-resilient development in Africa ..........................85

3.5. Conclusions and outlook for further work ..................................................... 89

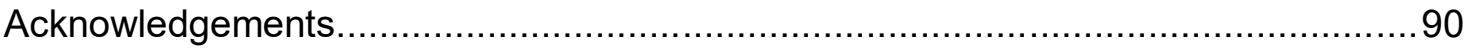

Annex 3. Overview of principal MDG and adaptation investments ........................... 91

4. Investment needs to achieve the Sustainable Development Goals in low- and lower-

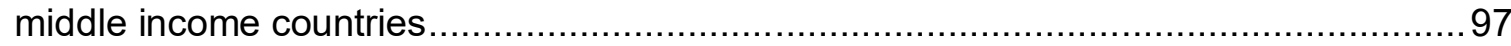

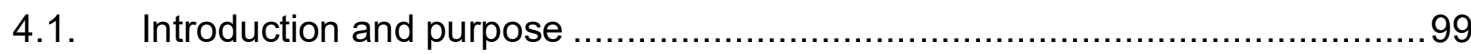

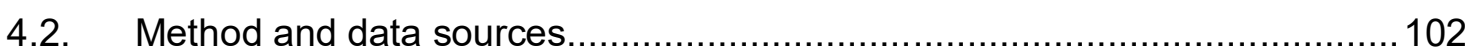

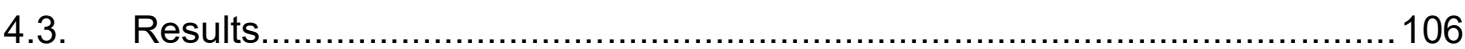

4.4. Conclusions and discussion ................................................................ 118

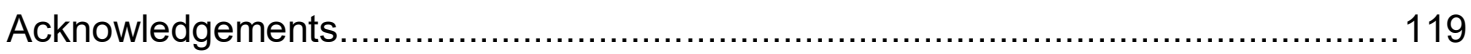

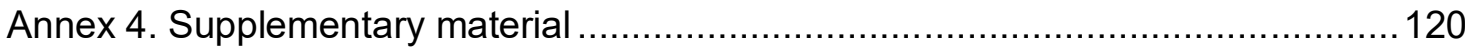

5. Lessons from the Global Fund to Fight HIVIAIDS, Tuberculosis and Malaria for the

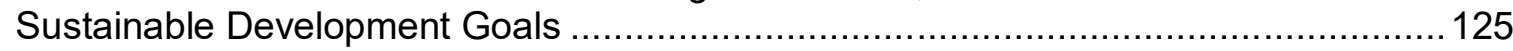

5.1. Transparency and independence underpin successes.......................... 127

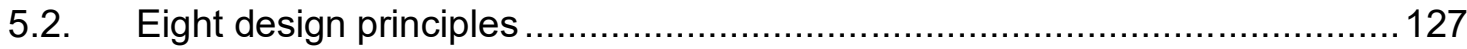

5.3. Initial doubts, lessons learnt ............................................................. 128 
On metrics and financing for the Sustainable Development Goals

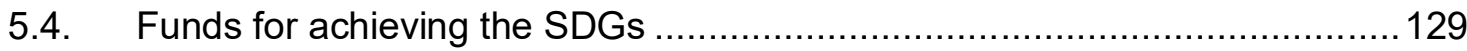

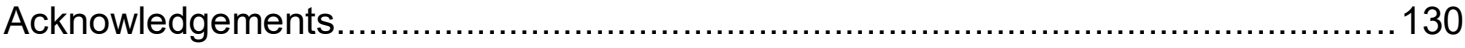

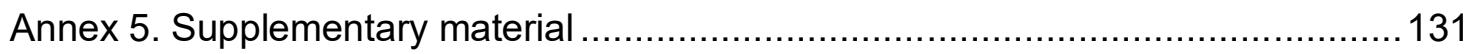

6. The role of the Technical Review Panel of the Global Fund: An analysis of grant

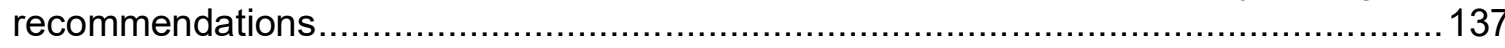

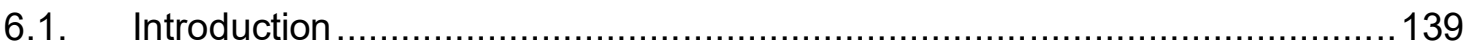

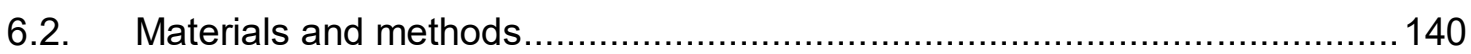

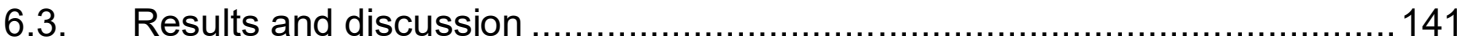

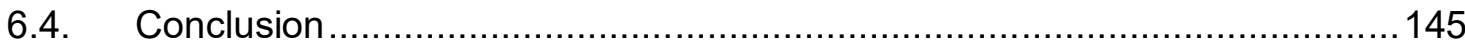

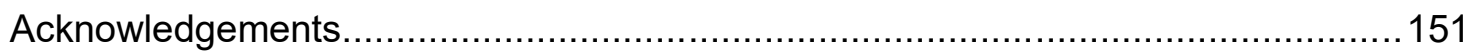

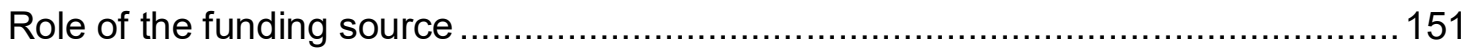

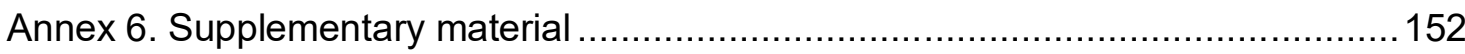

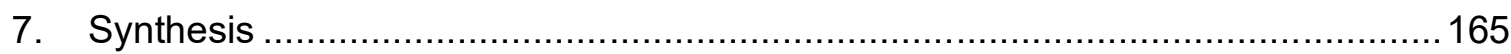

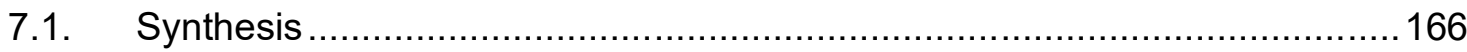

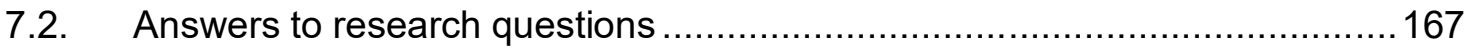

7.3. Evaluation of methodologies and data ............................................ 175

7.4. Suggestions for further research ......................................................... 180

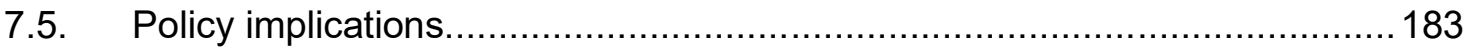

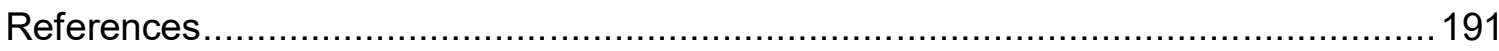

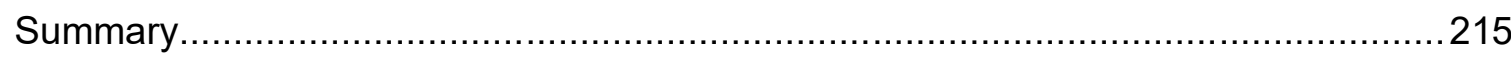

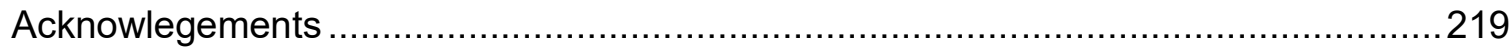

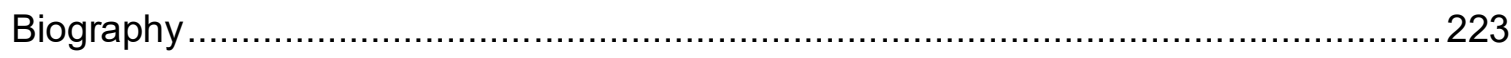

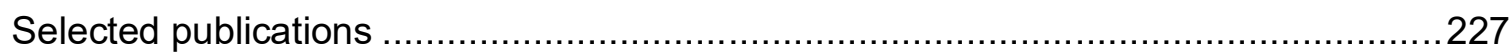

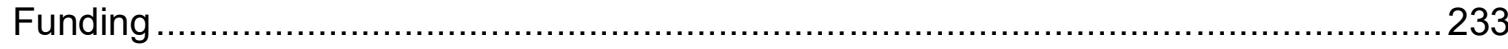

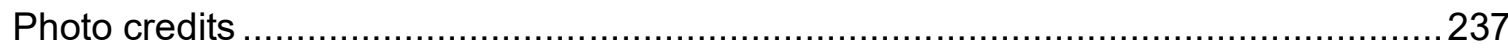




\section{List of tables}

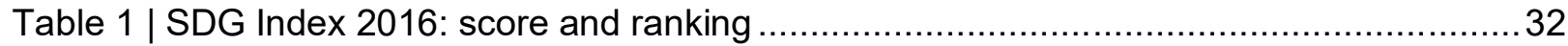

Table 2 | Relative performance on SDG-Index by region and income group.........................37

Table 3 | Deviation from average country SDG Index score by SDG ..................................38

Table 4 | Partial correlation coefficients with Cantril Ladder ................................................ 39

Table 5 | Principal SDG data issues and gaps................................................................ 41

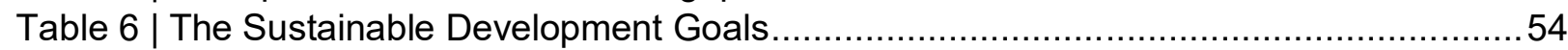

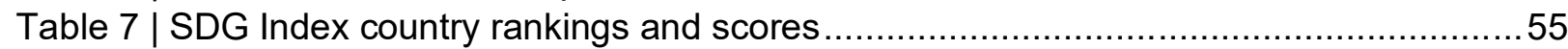

Table 8 | Indicators used in the SDG Index and Dashboards ...........................................5

Table 9 | Summary statistics for indicators included in SDG Index and Dashboards ..............59

Table 10 | Shapiro-Wilk test for normality on SDG Index indicators ...................................61

Table 11 | Indicator thresholds used in the SDG Index and Dashboards .............................63

Table 12 | Countries not included in the SDG Index and Dashboards .................................65

Table 13 | SDG Index rankings obtained using alternative scaling methods .........................66

Table 14 | SDG Indices obtained by arithmetic and geometric average ..............................69

Table 15 | Country scores by SDG. ......................................................................... 71

Table 16 | Sources and summary statistics of variables in partial correlation matrix ...............75

Table 17 | Significant partial correlates of subjective well-being ...................................... 75

Table 18 | Overview of cost estimates (\$bn p.a. over the period 2010-2020)........................86

Table 19 | Sources and assumptions on adaptation cost estimates ..................................... 87

Table 20 | MDG and adaptation interventions for education ............................................. 91

Table 21 | MDG and adaptation interventions for health .............................................. 92

Table 22 | MDG and adaptation interventions for agriculture, food security, nutrition...............93

Table 23 | MDG and adaptation interventions for infrastructure, trade facilitation ...................94

Table 24 | MDG and adaptation interventions for other development needs .........................95

Table 25 | Summary of sector needs assessments and adjustments made ........................ 105

Table 26 | Incremental annual investment needs in LICs and LMICs ................................114

Table 27 | Principal sources for needs assessments and adjustments made .....................120

Table 28 | Largest channels for Development Assistance for Health 2003-2015 .................133

Table 29 | Predictors of requested funding volumes per round, by disease ........................ 147

Table 30 | Predictors for TRP proposal ratings, by disease ............................................ 148

Table 31 | Predictors for total per capita Global Fund funding, by disease ......................... 149

Table 32 | Predictors for Phase 1 performance rating, by disease ..................................... 150

Table 33 | Codebook for regression variables .............................................................. 155

Table 34 | Summary statistics for Regression 1 and Regression 2, by disease ................... 156

Table 35 | Summary statistics for variables in Regression 3, by disease ............................. 157

Table 36 | Summary statistics for variables in Regression 4, by disease ........................... 158

Table 37 | Robustness of predictors of In(funding request per capita), by disease ................159

Table 38 | Robustness of predictors for TRP rating, by disease ................................... 160

Table 39 | Robustness of predictors for In(total Global Fund funding pc), by disease ...........161

Table 40 | Robustness of predictors for average Phase 1 performance, by disease .............162

\section{List of figures}

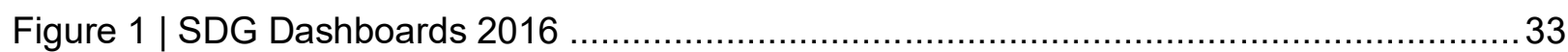

Figure 2 | Correlation of SDG Index with other common development indices...................... 35

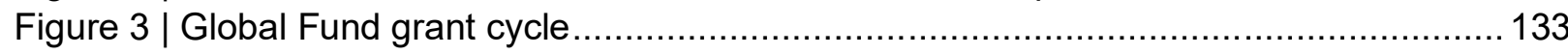


On metrics and financing for the Sustainable Development Goals 


\section{Chapter 1}

\section{Introduction}

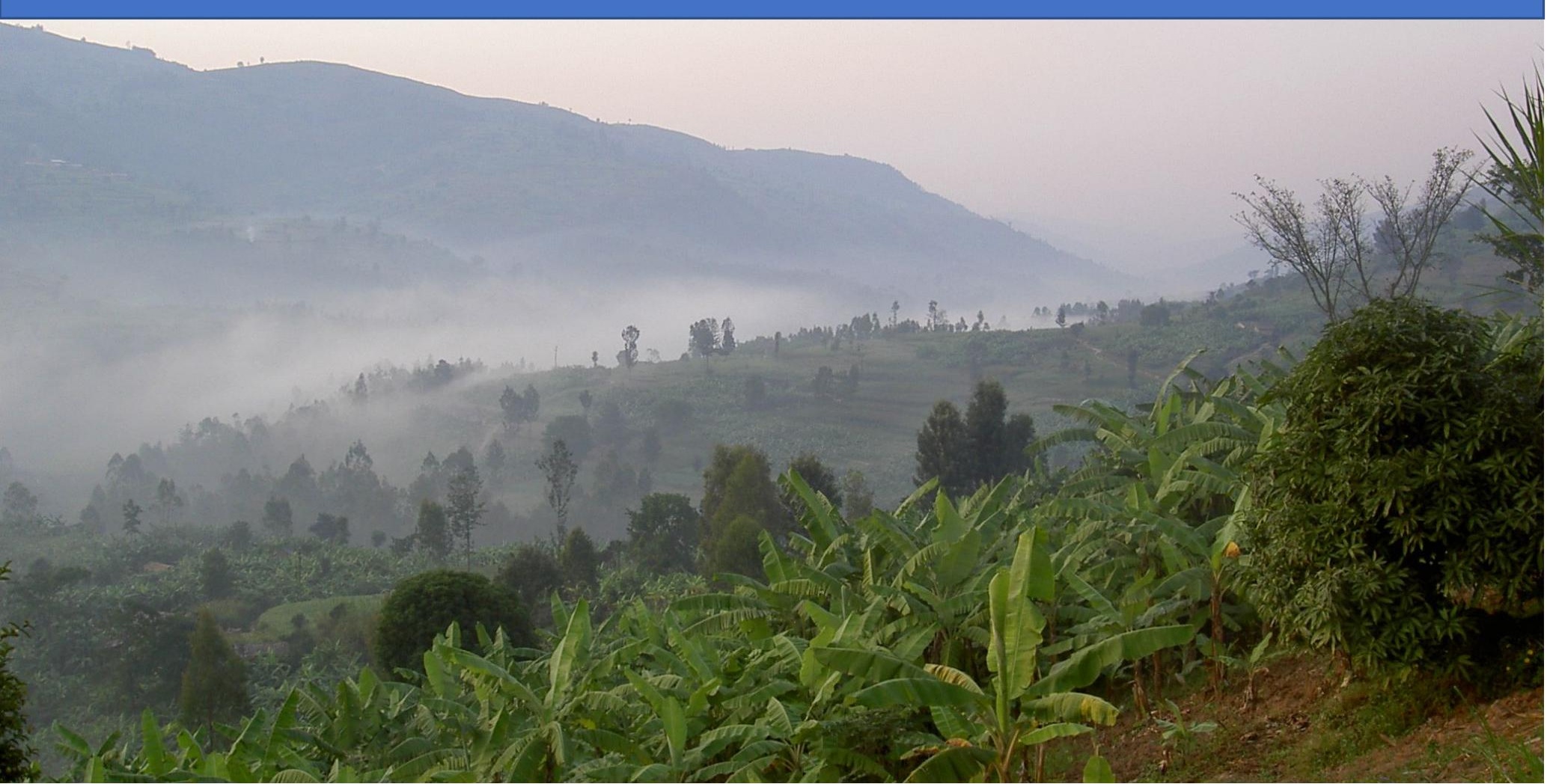




\subsection{Background}

The world has experienced unprecedented growth in average per capita incomes over the last 50 years, but many countries continue to face deep economic, social, and/or environmental challenges. These include persistent extreme poverty, poor outcomes in human health and education, widespread malnutrition, high inequality measured by income or other characteristics, poor access to infrastructure, growing water stress, the degradation of terrestrial and marine ecosystems, pollution, and climate change. Under business-as-usual trajectories, the environmental challenges in particular are expected to worsen significantly (Sachs, 2015; van Vuuren et al., 2015). Increased international cooperation is required to address these challenges since many operate at planetary scales (Rockström et al., 2009; Steffen et al., 2015) while others require technological and financial support from rich to poor countries to complement sound domestic policies (CMH, 2003; Sachs et al., 2004a; UN Millennium Project, 2005). Similarly, social exclusion is widening across most countries (UNDP, 2015; Helliwell et al., 2016b).

Since these challenges are interlinked they require integrated analyses and policy recommendations. To this end, the concept of 'sustainable development' was introduced by the Brundtland Commission as development that "meets the needs of the present without compromising the ability of future generations to meet their own needs" (Brundtland et al., 1987). One strand of applied welfare analysis operationalizes the Brundtland definition by estimating adjusted or genuine savings rates (Pearce and Atkinson, 1993; Hamilton and Clemens, 1999; World Bank, 2011). The 'genuine saving rate' adjusts the definition of the saving rates in national accounts by including human capital, natural capital, and the depletion of natural resources, such as oil, gas, or timber. In this way researchers can ask whether national saving is high enough to ensure rising living standards, comprehensively measured to include dimensions beyond income. Under this interpretation, high genuine savings rates suggest sustainable development.

Arrow et al. (2012) introduce the closely related concept of inclusive wealth (IW), which describes the value of future wellbeing $(\mathrm{V})$, defined as the integral of discounted future true consumption. Inclusive wealth is a function of various forms of capital (human capital, natural capital, health capital, and reproducible business capital) weighted by their respective shadow prices. Genuine saving may then be equated with the rise in IW. Countries with a decline in IW are dis-saving, typically because some of what is currently counted as national income is in fact the depletion of natural capital.

The Brundtland definition of sustainable development requires that $\mathrm{dV}>0$, that is a rise in the discounted future value of true consumption. In turn, $d V>0$ requires positive 'genuine saving' in order to raise inclusive wealth. IW can be calculated, at least in part, to see whether a society is achieving sustainable development in the Brundtland sense, though the data challenges to this exercise are substantial, particularly relating to the definition of a discount rate and the aggregation across different types of capital (Victor, 1991; Arrow et al., 2012, 2013; UNU-IHDP and UNEP, 2016).

Instead of asking whether $\mathrm{dV}>0$ (the Brundtland test), policymakers increasingly ask how the composition and scale of investment profiles need to change for an economy to achieve time-bound targets relating to the economy, social inclusion, and the environment (United Nations, 1994, 2012a; SDSN, 2013). This is a more stringent test than $\mathrm{dV}>0$, particularly for low-income countries where inclusive wealth will need to rise quickly to provide income, social services, infrastructure, and environmental services. In some sectors, such as health and education, the composition of current investments will need to change little, but the scale will need to increase substantially, so the focus 
is on increasing the volume of investments. In other areas, such as the decarbonization of the energy system, the composition of investments must also change significantly alongside an increase in investment flows.

Following the 1992 Rio Earth Summit, the most common tools for international cooperation on sustainable development have been legally binding environmental conventions, such as the United Nations Framework Convention on Climate Change (UNFCCC) and the Convention on Biological Diversity (CBD). Yet during the first 20 years following their adoption, the conventions did not lead to any lasting change on the underlying environmental challenges, which continued to worsen steadily (Tollefson and Gilbert, 2012). An important exception is the Montreal Protocol on Ozone-depleting Substances, which has been credited with reversing the loss of stratospheric ozone (Chipperfield et al., 2015), but it is unclear whether this success can be replicated for other more complex challenges, such as climate change.

In 2001, building on the Millennium Declaration (United Nations, 2000), the United Nations introduced the Millennium Development Goals (MDGs) that expired in 2015. They constituted the first set of global development goals covering a broad range of sustainable development priorities (McArthur, 2013), although climate change and other key challenges were notably omitted. In contrast to environmental conventions, the MDGs were not legally binding, and did not have any formal government review and followup processes. Yet, they have been credited with mobilizing attention on the challenges of extreme poverty, hunger, illiteracy, and disease. The MDGs have spurred advances, particularly in health (Kassebaum et al., 2014; You et al., 2015; McArthur and Rasmussen, 2017). Access to improved water supply has also experienced faster progress, but in other areas the MDG period has not seen a significant acceleration of progress (McArthur and Rasmussen, 2017).

Although the MDGs were not formally adopted by UN Member States, they were officially recognized as the world's goals for addressing extreme poverty in all its forms at the 2005 World Summit in New York (McArthur, 2013, 2014). Starting with the United Nations and some bilateral donors, most development organizations, including the IMF (2008), adopted the MDGs as operational development goals. Driven in large parts by the perceived (partial) success of the MDGs, member states insisted that they negotiate the Sustainable Development Goals (SDGs) following the Rio +20 Summit (United Nations, 2012a). The resulting 17 SDGs (United Nations, 2015a) represent a political compromise by the 193 member states of the United Nations that has been critically reviewed (ICSU and ISSC, 2015) and raises major challenges of measurement, financing, and implementation. The SDGs are much broader than the MDGs in that they are very ambitious, and unlikely to be met under a business-as-usual pathway (Joshi et al., 2015; van Vuuren et al., 2015; Häyhä et al., 2016). Yet, contrary to the early years of the MDG period (McArthur, 2013), governments around the world are already adopting the SDGs as operational goals for sustainable development (United Nations, 2017).

The MDGs and the ensuing SDGs have accelerated a trend in the aid literature to place a greater focus on understanding the impact of aid on social outcomes (Temple, 2010). Originally, much of the literature concentrated on how aid affected economic growth and how donors could improve the targeting to remove binding constraints. (Burnside and Dollar, 2000; Collier and Dollar, 2004; Hausmann et al., 2008). With the advent of the MDGs researchers started to look into how aid could help improve health, education, and other outcomes (Boone, 1996; Sachs et al., 2004a; Sachs, 2005; Mishra and Newhouse, 2007; Banerjee, 2008; Dreher et al., 2008). 


\subsection{Problem definition and research questions}

This thesis contributes to understanding how progress towards the SDGs can be monitored, how investment needs for climate-resilient development and the SDGs can be estimated, and what lessons can be drawn for international financing mechanisms in support of the SDGs from the experience of the health sector under the MDGs. These issues cover important contemporary questions in the scientific and policy literature, as evidenced by the rapidly growing scientific literature on the SDGs to which this thesis contributes. By addressing five research questions, the thesis will test the hypotheses that the SDGs can be tracked with available data to establish baselines and identify sustainable development challenges at the country level; that the world has ample savings to mobilize the public and private financing for achieving the SDGs; and that the institutional innovations undertaken by the Global Fund to Fight HIVIAIDS, Tuberculosis and Malaria offer lessons for the design of international financing mechanisms for the SDGs.

\subsubsection{Baselines for the SDGs}

To apply the SDGs as operational targets for sustainable development, countries need to establish quantitative baselines, measure the distance they need to cover to achieve each goal, and track progress using a combination of input and outcome metrics (Lu et al., 2015; SDSN, 2015a; Nilsson et al., 2016; Sachs et al., 2016c). The UN Statistical Commission has recommended a first set of 230 indicators to measure achievement of the SDGs, but many suggested indicators lack comprehensive, cross-country data and some even lack agreed statistical definitions (IAEG-SDGs, 2016a). Moreover, some of the proposed indicators have been criticized for lack of scientific rigor and appropriateness (Nilsson et al., 2016).

To track progress towards national development objectives, governments traditionally rely on official statistics collected and validated through national statistical offices and other government institutions. Yet, official data do not adequately track many of the SDGs, particularly in the areas of sustainable development and social inclusion (Espey et al., 2015; SDSN, 2015a). Current investments in expanding statistical systems are inadequate (United Nations, 2016a).

In the meantime, scientifically robust data tools are needed to help operationalize the SDGs at the global, regional, national, and sub-national levels and to begin a process of data-driven and evidence-based implementation and follow-up. The MDG experience in the health sector suggests that such data tools need to be nationally relevant to support policy design and be comparable across countries to promote international benchmarking, accelerate learning on the measurement of the SDGs, and help establish the SDGs as widely used development goals (Murray and Chambers, 2015; Schmidt-Traub and Sachs, 2015; McArthur and Rasmussen, 2017).

Reporting against a large number of indicators alone, whilst providing much detail about specific domains, eventually leaves open the question of how to measure the aggregate performance of a country. Composite indices have well-known weaknesses (OECD and JRC, 2016), but they can synthesize complex information into a single number and may be more effective in stimulating public debates than a large number of individual scores which could result in 'cherry picking' (Dasgupta and Mäler, 2000; United Nations, 2016a). To inform policies for the achievement of complex integrated goals, a combination of composite measures and dashboards are therefore needed for the SDGs. Prominent examples of such composite indices in recent years include the Global Burden of Disease index for the health SDGs (GBD, 2016), the Environmental Performance Index (Hsu et al., 2016), the Ocean Health Index (Halpern et al., 
2012), the Human Development Index (UNDP, 2016a), the PISA education assessment (OECD, 2012), the Global Competitiveness Index (Schwab and Sala-i-Martín, 2016), the Global Peace Index (IEP, 2016), the Index of Economic Freedom (Miller, 2015), and many others. Some of these composite indices have had significant impact in drawing political attention (Waldow, 2009; Kroll, 2011), guiding policies, fostering learning across countries, and promoting more research (Dervis and Klugman, 2011; Wiseman, 2013). Yet, only the health-related SDG Index by the Global Burden of Disease collaboration, was specifically designed to track a part of the SDG agenda.

The absence of SDG-focused indices generates the first research question considered in this thesis:

Research Question 1: How to identify SDG implementation priorities for each country based on a measurement of countries' distance from achieving the goals?

Several sub-questions need to be considered to address Research Question 1. They include (i) Which official and unofficial data are available today to track the SDGs and how can it be harmonized to quantify gaps in SDG achievement? (ii) How can data be aggregated within and across SDGs to support scientific inquiries into the SDGs and to guide policymakers at national, regional, and global levels? (iii) How can countries' progress towards implementing the 17 SDGs be assessed, compared, and tracked over time? (iv) Which data gaps need to be filled to support better monitoring and facilitate peer-learning between countries regarding policies that help achieve the SDGs?

\subsubsection{Investment needs for climate-resilient development}

Sector needs assessments estimate the scale and composition of public and private investment needs for a country or a set of countries to achieve specific development outcomes, such as the MDGs or SDGs. They are anchored in the research literature that investigates how public investments complemented by aid promote social and other targeted outcomes (Temple, 2010). Here, the term 'investments' includes capital and operating expenditure needed to achieve the outcomes laid out in the goals. Methodologies for needs assessments, sometimes referred to as costings, were pioneered in the early 2000s for the health sector $(\mathrm{CMH}, 2003)$, where they have since improved significantly (Waage et al., 2010; Jamison et al., 2013). Over time, they have been developed for most investment areas covered by the MDGs (Commission for Africa, 2005; UN Millennium Project, 2005; Bourguignon et al., 2008; Ki-Moon et al., 2008).

Needs assessments can support analyses and policies to achieve international development goals in four ways. First, they offer a methodology for understanding the investments required to achieve the goals and for identifying knowledge gaps in the understanding of implementation strategies or 'production functions' for each goal. Second, results from needs assessment provide a basis for determining how the goals can be financed through a combination of private investments, domestic public finance, concessional and non-concessional international public finance. Third, the scale and nature of projected investment needs obtained through needs assessments can help design medium-term expenditure frameworks and macroeconomic management strategies to accommodate large changes in the scale and composition of public and private spending, as illustrated by the IMF for the MDGs in Africa (IMF, 2008; Mongardini and Samake, 2009). Fourth, needs assessments can support resource mobilization and provide an accountability framework for implementation, as demonstrated by recent successful replenishment rounds of the International Development Association (IDA, 2013), the Global Fund to Fight AIDS, Tuberculosis and Malaria (GFATM, 
2015a), the Global Alliance for Vaccines and Immunization (Gavi, 2014), and the Global Financing Facility (GFF, 2015). Each of these financing mechanisms conducted needs assessments to determine the volume of required resources, identify results that would be achieved with greater financing, and propose an accountability framework to track the effective use of additional resources.

Needs assessments for global goals are imperfect analytical tools and therefore controversial. Some researchers challenge the focus on investment needs, arguing that sound policies and good governance are more important (Devarajan et al., 2002; ODI et al., 2015). Yet, the MDG experience shows that needs assessments can address policy issues for achieving global goals, such as removing user fees for health services (WHO, 2010; Jamison et al., 2013; Chatham House, 2014), subsidies for cooking fuels (Pachauri et al., 2013), or providing feed-in tariffs for renewable energy (NCEC, 2014). A second critique is that needs assessments do not adequately consider absorptive capacity constraints (Clemens et al., 2007), defined as the ability to scale up public investments efficiently at the sector and macroeconomic level. At the sector level, needs assessments help identify capacity constraints (e.g. human resources, management and monitoring systems, infrastructure) and outline ways in which they can be addressed. At the macroeconomic level, foreign currency inflows may lead to real exchange rate appreciations and shift domestic investments away from the tradable sector. As shown by the IMF, detailed needs assessments are a prerequisite to understanding how countries need to adjust their macroeconomic frameworks and policies to mitigate adverse consequences from foreign currency inflows (Prati et al., 2003; IMF, 2008; Mongardini and Samake, 2009). A third critique is that needs assessments tend to be partial-equilibrium analyses and it is impossible to predict unit costs over extended periods of time (Bourguignon et al. 2008). These are fundamental challenges that require needs assessments to be considered in conjunction with dynamic economy-wide tools.

Economic development and adaptation to climate change are closely linked, nowhere more so than in Africa. Africa faces the biggest development challenges of any continent (Sachs et al., 2004a; Commission for Africa, 2005). It is also the region that is most vulnerable to climate change (Collier et al., 2008; Barr et al., 2010; World Bank, 2010a; IPCC, 2014). Some (McGray et al., 2007; Klein and Persson, 2008) argue that vulnerability to climate change can be reduced through measures that range from pure development to pure adaptation. Fankhauser and Burton (Fankhauser and Burton, 2011) put development-style projects at the core of spending adaptation finance wisely. Others discuss how different growth paths both affect and are affected by climate vulnerability (Benson and Clay, 1998; Dell et al., 2009; Noy, 2009; Vivid Economics, 2010). Adaptive capacity - the ability to deal with climate events - is known to be driven pre-dominantly by indicators of development such as health, literacy, income and institutional quality (Tol and Yohe, 2007; Barr et al., 2010).

Yet, in much of the policy discourse adaptation is still treated as a stand-alone issue with few or no links to other development challenges. Analyses of adaptation needs tend to see adaptation as an incremental activity bolted onto a 'business as usual' development path (UNFCCC, 2007; Agrawala and Fankhauser, 2008; Parry et al., 2009; World Bank, 2010a), and there is a lack of clarity on how finance for development and adaptation relate to one another and can be accounted for (Atteridge et al., 2009; Buchner et al., 2014; OECD and CPI, 2015; Donner et al., 2016). This motivates the second research question considered in this thesis: 
Research Question 2: How can interventions and associated financing needs for development and climate change adaptation be integrated to estimate the combined financing needs in Africa?

To answer this question, we will use Africa as an example and use the MDGs, as considered in the academic and policy literature, to define the scope of 'development' in the absence of climate change adaptation measures. Notwithstanding the wellknown analytical shortcomings in the estimation of resource needs (Ki-Moon et al., 2008; Parry et al., 2009; Fankhauser, 2010), addressing this question will help arrive at an integrated framework for development and climate financing needs. Gradual refinements of the numbers and the filling-in of remaining analytical gaps will over time lead to a better assessment of the resources Africa requires to pursue climate-resilient development (Stern, 2009a), including financing needs for climate change mitigation (Bataille et al., 2016; Sachs et al., 2016c). By specifying financing needs for adaptation and development in one integrated framework, the analysis will also help define the financial 'additionality' of climate adaptation financing, a central question in the policy and research literature (Stern, 2007; World Bank, 2010a; Buchner et al., 2014; OECD and $\mathrm{CPI}, 2015)$.

\subsubsection{Public and private investment needs and financing for the SDGs}

The four motivations for needs assessments identified in the previous section (understanding the investments required to achieve the goals and knowledge gaps relating to the 'production functions' for each goal; developing a financing strategy comprising domestic and international financing from public and private sources; designing medium-term expenditure frameworks and macroeconomic management strategies to accommodate large changes in the scale and composition of public and private spending; and supporting resource mobilization and providing an accountability framework for implementation) also apply to the SDGs. Indeed, international policy frameworks like the Addis Ababa Action Agenda on Financing for Development (United Nations, $2015 b$ ) cannot be implemented without quantifying SDG resource needs and developing adequate financing strategies (SDSN, 2015b). Owing to the novelty of the SDGs, investigations into the resources required for achieving all goals have yet to be conducted.

Three aspects of the SDGs make needs assessments more complex to conduct than for the MDGs. First, the SDGs represent a broader, more complex, and more ambitious agenda than the MDGs with multiple interactions across goals and targets (Lu et al., 2015; Nilsson et al., 2016). Assessing investment needs for the SDGs therefore requires that the 17 goals be translated into discrete investment areas and that interactions as well as overlaps across investment areas be addressed. This also makes it harder to separate the effect of aid on economic growth from the achievement of targeted investment objectives in social sectors, environmental sustainability, and other SDG priorities.

Second, the SDGs apply to all countries (United Nations, 2015a) while the MDGs were primarily an agenda for the poorest countries (UN Millennium Project, 2005). Therefore, needs assessments for the SDGs must distinguish between differences in the type and magnitude of incremental investment needs for the SDGs and countries' ability to finance the goals using domestic and international resources from public and private sources. Poor developing countries will need to significantly increase public and private investments in the economic, social, and environmental dimensions of sustainable development (UNCTAD, 2014; UNTT, 2013). In turn, high-income countries 
will primarily need to redirect public and private expenditure coupled with modest incremental expenditure, as documented, for example, for the transition towards lowcarbon energy systems (GEA, 2012; NCEC, 2014; Bataille et al., 2016). Upper-middleincome countries present an intermediate set of financing challenges.

Finally, a greater share of investments for the SDGs can be privately financed (UNCTAD, 2014). While MDG needs assessments (e.g. CMH (2001), UN Millennium Project $(2004,2005)$, Bourguignon et al. (2008), and Ki-moon et al. (2008)) focused almost exclusively on public financing of the MDGs, needs assessments for the SDGs require the explicit consideration of private financing.

Available needs assessments reviewed by UNTT (2013) demonstrate that not all SDG sectors have developed a clear understanding of the types and scale of investment needs. For example, some sectors do not model the change in composition of investments needed to achieve the goals. Moreover, there is no agreed methodology for aggregating investment needs across the SDGs, which leads to large ranges in projected investment needs (UNTT, 2013).

To address some of these gaps and to define a research agenda for more robust assessments of SDG investment needs, we pursue a third research question in this thesis.

Research Question 3: What are the private and public financing needs from domestic and international sources to achieve the SDGs in low- and lower-middle-income countries?

Addressing this research question will require a synthesis of needs assessment studies to address three additional sub-questions: First, what are the principal investment areas for the SDGs that cover the full range of the goals and can guide SDG needs assessments at global, regional, national, and sub-national levels? Second, how can available needs assessment studies be made comparable and be aggregated into an overall assessment? Third, how can the combined SDG investment needs be financed using domestic and international resources from public and private sources?

Our analysis in response to this question will show that substantial gaps exist in available needs assessments for the SDGs. These cannot be fully addressed within the scope of this thesis, so we do not aim for definitive answers to Research Question 3. Instead we will focus on synthesizing the available literature and on developing a framework for conducting SDG needs assessments at distinct levels of spatial aggregation. We will seek to identify a research agenda to support more robust assessments of public and private investment needs for the SDGs.

To further define the scope of the analysis, we will focus on incremental investment needs for the SDGs. We will therefore limit ourselves to assessing SDG investment needs in low- and lower-middle-income countries, as defined by the World Bank (2016a). Changes in the composition of expenditure, as primarily needed in wealthier countries, will require different methodologies and data sources. The focus on low- and lower-middle-income countries is further motivated by the fact that these countries will not be able to finance the SDGs from domestic resources alone (UNCTAD, 2014). They will require greater public and/or private finance from external sources, and increasing such international finance is a central aim of Agenda 2030 and the Addis Ababa Action Agenda (United Nations, 2015a, 2015b). To this end, policymakers require rigorous SDG needs assessments. 


\subsubsection{Lessons from the Global Fund for the SDGs}

Building on the analysis of domestic and international financing needs for sustainable development in response to research questions 2 and 3 , we will turn to the question of how concessional international development financing can be delivered across a spectrum of country settings, defined by per capita income, ecological fragility, governance, human capital, and other country characteristics. We are interested in how aid can achieve sector investment targets, as opposed to economic growth (see Temple (2010) for a discussion of these strands of the literature).

The health sector has been among the first to pioneer long-term global goals $(\mathrm{CMH}$, 2001; UN Millennium Project, 2005) and to devise national and global investment strategies to achieve them (Schmidt-Traub and Sachs, 2015). It has also experienced the greatest acceleration in progress under the MDGs (McArthur and Rasmussen, 2017). For these reasons, it is instructive to consider the experience of the health sector and more specifically the fight against the major infectious diseases (HIVIAIDS, TB, and malaria), which experienced the greatest acceleration in progress under the MDGs. Clearly, care must be exercised in applying lessons from health to other SDG investment areas, since the latter may present different and highly complex implementation and investment challenges.

By 2000 prevalence and incidence rates of the three infectious diseases had reached unprecedented levels, particularly in sub-Saharan Africa. This health crisis threatened the future of many developing countries (Adams et al., 2001; CMH, 2003) even though medical and public health interventions, such as anti-retroviral therapy, were available to control and treat each disease in high-income countries. Scientific assessments of treatment options for HIVIAIDS made no reference to anti-retroviral therapy (Binswanger, 2000). Similarly, there was no concerted effort to scale up proven lifesaving interventions against malaria and TB.

In response to public pressure (Adams et al., 2001; Schwartländer et al., 2006; Behrman, 2008) and following the call for action by UN Secretary-General Kofi Annan (United Nations, 2001), leaders at the $2001 \mathrm{G} 7$ Summit in Genoa endorsed the idea of a global fund for the three diseases. After an extensive technical design process, the Global Fund to Fight HIVIAIDS, Tuberculosis, and Malaria ('Global Fund') was launched in late 2001 and became fully operational in early 2002. One year earlier, the smaller Global Alliance for Vaccines and Immunization (Gavi) had been created to finance the development and delivery of vaccines in developing countries.

Within a few years the Global Fund became the dominant provider of international finance for malaria and tuberculosis and the second largest aid provider for HIVIAIDS after the US President's Emergency Program for AIDS in Africa (PEPFAR). Together with Gavi these two programs accounted for the bulk of the large increase in development assistance for health during the MDG period (IHME, 2016). No other MDG sector experienced a similarly sharp increase in international development assistance under the MDGs (Schmidt-Traub and Sachs, 2015). Programs supported by the Global Fund are credited with saving millions of lives (GFATM, 2015b). As we will consider in this thesis, this success was far from guaranteed since at the time many observers were skeptical of the chances of success for this new institution.

The Global Fund has unique institutional design features (GFATM, 2001; Triponel, 2010) and a business model that sets it apart from other major multilateral funders in the health sector (Shakow, 2006). It has undergone many external evaluations, (e.g. Macro International (2009), French Ministry of Foreign Affairs (2013), Norwegian Min- 
istry of Foreign Affairs (2013), DFID (2016)). A substantial scientific literature, predominantly published in The Lancet, has covered different aspects of its business model, such as performance-based funding (Katz et al., 2010; Fan et al., 2013, 2014; Glassman et al., 2013), its impact on health systems (Samb et al., 2009; Bowser et al., 2014), involvement of civil society (Harmer et al., 2012; Bridge et al., 2016), drug pricing (Stover et al., 2011; Zelman et al., 2014), its role in fragile countries (Bornemisza et al., 2010), and the promotion of implementation research and management learning (van Kerkhoff and Szlezák, 2006, 2010).

However, the literature tends to focus on individual components of the Global Fund without linking them to the institution's key design principles, and without considering how they might interact to explain the apparent success of the Fund. Such analyses need to be considered in the context of the development economics literature on optimal aid allocation (World Bank, 1998; Collier and Dollar, 2002; Van de Walle, 2005; Wood, 2008) and conditionality (Collier, 2008; Temple, 2010). The traditional aid allocation literature focuses on how aid volumes can be optimally allocated among countries without investigating how effective demand can be generated from recipient countries. Yet, this was the challenge faced by the Global Fund in 2001: In the face of a public health emergency in many developing countries there was significant political pressure to increase investments. Yet recipient countries did not have strong investment programs in place for scaling up investments in HIVIAIDS, TB, and malaria, so effective demand had to be generated quickly across a broad spectrum of low-income, middle-income countries, and countries emerging from conflict. Another critical question not considered in the literature on the Global Fund, is the impact of aid fragmentation and demands from multiple donors on recipient countries (Acharya et al., 2006; Easterly, 2007). Moreover, analyses do not consider lessons from the Global Fund for other SDG investment priorities and associated multilateral financing mechanisms. This leads to our fourth research question:

Research Question 4: To what extent do the key design principles of the Global Fund explain the institution's success in delivering $\$ 35$ billion in incremental health financing for novel and complex programs including in poorly governed countries and countries emerging from conflict?

Addressing this question will allow us to explore lessons from the Global Fund for the financing of other SDG priorities in the health sector and beyond. This information will help researchers and practitioners consider options for improving the effectiveness and scale of other multilateral financing mechanism, such as the Green Climate Fund, the Global Environment Facility, the Global Partnership for Education, and the World Bank, as well as bilateral mechanisms, such as PEPFAR and the U.S. President's Malaria Initiative (PMI) launched by President George W. Bush.

\subsubsection{Reconciling country ownership with independent technical ap- praisal}

The Global Fund was established in 2001 against the backdrop of a global health emergency across developing countries and high political expectations of rapid scaling-up of proven health interventions. The health crisis generated pressure to disburse large volumes of resources quickly (HLIRP, 2011). Key medical and public health interventions to control the diseases were well understood and had been deployed in small projects in poor countries (Adams et al., 2001; Farmer et al., 2001), but there was little knowledge of how to deploy interventions at scale in developing countries (Binswanger, 2000; HLIRP, 2011). Some interventions, such as anti-retroviral therapy and treatments for multi-drug-resistant TB were expensive and complex, making their 
large-scale deployment in poor countries particularly difficult (Schwartländer et al., 2006; Stover et al., 2011). Other interventions, such as artemisinin-based combination therapy (ACT), long-lasting insecticide-treated nets (LINs), or rapid diagnostic tests for malaria (RDTs), had yet to be developed or be deployed in developing countries, sometimes owing to their higher cost relative to less effective interventions (Mutabingwa, 2005; Noor et al., 2009; Zhao et al., 2012). The operational and managerial knowledge for how to design, implement, and monitor complex programs in different country operating environments was largely unavailable (van Kerkhoff and Szlezák, 2010).

The need to respond quickly to the global health crisis and to meet high expectations from world leaders, combined with the lack of clarity of how to go about such a scaling up, forced the experts designing the Global Fund to reconsider two tensions and tradeoff that have been well documented in the technical and policy literature on aid (Kharas, 2007; OECD, 2008; Temple, 2010; Chandy and Kharas, 2011), and especially on performance-based aid allocation (Adam et al., 2004; Barder and Birdsall, 2006; Wood, 2008; Fan et al., 2013; Glassman et al., 2013). The first concerns the tension between country ownership and the need to ensure effective use of scarce resources, consistent with medical best practice in the case of health. This is sometimes referred to as the tension between conditionality and autonomy (Temple, 2010): On the one hand, addressing complex diseases, such as HIVIAIDS, TB, and malaria requires strong political leadership at the country level to raise awareness, tackle taboos, and ensure the effective design, implementation, and monitoring of complex disease management programs. This could not be achieved without strong local leadership and the involvement of different groups of stakeholders (Atun and Kazatchkine, 2010; HLIRP, 2011). On the other hand, most health systems required profound changes in the way they tackled the disease to comply with medical best practice and ensure effective resource use, as illustrated by the prevailing practice of HIVIAIDS and malaria control in China (Wang et al., 2014; Minghui et al., 2015), TB control in Russia (Perelman, 2000), or HIVIAIDS in South Africa (Fassin and Schneider, 2003).

Second, the Global Fund framers grappled with the tension between combining bottom-up demand-led programming of resources with the imperative to channel resources to the countries most in need. This tension was particularly important for the Global Fund since some of these countries had low capacity to formulate, implement, and monitor complex disease control programs. Demand-led programming was critical for the aforementioned reasons of national ownership, but also because it was seen as the only avenue towards the rapid scaling-up of proven interventions expected by policymakers in 2001 and towards promoting the innovation in program design and execution required to address major implementation research gaps (HLIRP, 2011). Yet, it was not clear whether demand-led programming could ensure that resources went to the poorest countries, which commonly had weak implementation capacities. This tension and the resulting implementation challenges have been reviewed widely in the literature on optimal aid allocation (Alesina and Dollar, 2000; Bourguignon et al., 2009; Temple, 2010).

To address these two tensions, the Global Fund introduced the independent Technical Review Panel (TRP) comprising scientists drawn from a broad range of medical specialties. Countries were invited to submit program proposals in key disease categories, which the TRP then reviewed and recommended for funding or rejection to the Board of the Global Fund. The Board could only approve or reject the TRP's recommendation in toto to avoid political interference with financing decisions regarding individual countries. Since the creation of the Global Fund, the Board has always approved the TRP recommendations in full, and every board-approved program has been fully funded. As a result, the TRP has effectively determined Global Fund funding allocations that 
had to balance country ownership, compliance with medical best practice, and the need to direct resources to countries that needed them most.

Moreover, the TRP played an important role in promoting learning on how to design and implement national-scale health programs. It identified weaknesses that recommended approvals needed to address and encouraged some rejected proposals to be resubmitted after addressing weaknesses identified during the review process. The TRP also documented lessons learnt from each funding round and discussed them with technical cooperation organizations that assisted countries in the development of proposals to the Global Fund. In this way lessons learnt during the review of funding proposals were identified, made public, and disseminated.

Despite its centrality to the working of the Global Fund and its uniqueness among multilateral development financing mechanisms, the TRP has not been subjected to rigorous scientific assessments. Moreover, the development economics literature lacks detailed institutional assessments of how conditionality and autonomy can be reconciled, including by investigating intermediate channels and mechanisms (Temple, 2010). This leads us to the fifth research question considered in this thesis:

Research Question 5: Did the Global Fund's Technical Review Panel (TRP) make funding recommendations that were in line with the objective of allocating funding to countries most in need?

We will consider several sub-questions in relation to Research Question 5: (i) Did the TRP make funding decisions consistent with the principle of independent technical review or is there evidence of systematic bias in its decision making, including financial suppression of larger proposals, as is common in the allocation of aid in other sectors (Temple, 2010; Bourguignon et al., 2009)? (ii) Has the quality of proposals submitted to the TRP improved over time or is there other evidence of learning? (iii) Did fragile countries succeed in accessing Global Fund resources? (iv) What lessons can be learnt for other multilateral funding mechanisms?

Following the suspension of Round 11 in 2011, the Global Fund shifted away from a purely demand-led funding model where countries could apply for as much funding as they deemed necessary (HLIRP, 2011). Under the New Funding Model (now referred to simply as the 'Global Fund Funding Model'), countries could apply for funding allocations determined ex ante based on disease burden and income per capita (GFATM, 2013). A small window of incentive funding maintained elements of the demand-led model of the initial rounds-based mechanism, but following the end of the roundsbased mechanism in 2010, the TRP's role shifted fundamentally from recommending funding allocations to countries towards identifying the highest priority interventions within a set funding envelope for each country. For this reason, we limit our analysis to the rounds-based mechanism from 2002 to 2010.

\subsection{Methods}

This thesis applies several empirical methods drawing on a broad spectrum of the scientific and policy literature to address the five research questions. Chapter 2 constructs a new composite SDG Index and Dashboards drawing on methodologies described in Booysen (2002), GBD (2016), and OECD and JRC (2016). It considers the data availability, statistical adequacy, and policy relevance of the more than 230 official SDG indicators (IAEG-SDGs, 2016b) endorsed by the UN Statistical Commission and fills indicator gaps using other published metrics. Data for each indicator are normalized to quantify SDG baselines, track progress towards the goals, and permit data 
aggregation within and across SDGs. Drawing on the constant elasticity of substitution (CES) function, the chapter considers the theoretical and empirical implications of different aggregation techniques on the results of an overall SDG Index. Separate SDG Dashboards are developed to aggregate data on SDG performance by goal. Owing to limited data availability, the preliminary SDG Index and Dashboards comprise 149 out of 193 member states of the United Nations. Sufficient data are unavailable for many small-island countries with populations smaller than 1 million and countries in conflict or recovering from conflict.

To test the usefulness of the SDG Index, we consider its correlation with widely-used development indicators, including per capita gross domestic project (GDP); the Human Development Index (HDI) that measures health, education, and income outcomes (UNDP, 2016a); the Global Competitiveness Index, as a common measure of countries' economic competitiveness (Schwab and Sala-i-Martín, 2016); the Index of Economic Freedom, which estimates countries' governance (Miller, 2015); the Environmental Performance Index, which comprises a broad set of environmental indicators (Hsu et al., 2016); and the Global Peace Index, as a broad measure of peace and conflict (IEP, 2016). We also illustrate the analytical usefulness of the SDG Index by conducing partial correlation analyses of subjective well-being, a key aggregate objective of public policy (Stiglitz et al., 2009; OECD, 2013), with the SDG Index and other widely used synthetic cross-country indices. The partial correlations control for the two main macroeconomic correlates of subjective well-being identified in the literature: per capita income and unemployment (Clark and Oswald, 1994; Delhey and Kroll, 2013). Finally, we consider issues involved in tracking SDG progress over time and identify data gaps emanating from this first version of the SDG Index and Dashboards.

In Chapter 3 we structure the analysis around the expenditure tables prepared by the MDG Africa Steering Group, which are derived from sector-level analyses and draw on the combined research and operational expertise of the African Development Bank, European Union, IMF, OECD, United Nations organizations, and the World Bank (KiMoon et al., 2008). Results presented by the authors provide a good basis for the analysis in this thesis since they are scalable to national and regional levels; macroeconomically sound, as shown by the IMF (2008); sector-based to support implementation and permit the gradual improvement of analysis; and benchmarked through the organizations' on-the-ground experiences. However, the MDG Africa Steering Group's findings do not address climate change adaptation and implicitly assume stable climatic conditions in Africa, which is highly unlikely to be the case (IPCC, 2014).

Based on a literature review we then identify and quantify key 'baseline' development interventions that have been omitted from the analysis of the MDG Africa Steering Group. These include the cost of humanitarian assistance, disaster reduction, and some capacity development. We then expand the analysis of the MDG Africa Steering Group to introduce climate change adaptation. This is done in two steps. First, we identify the nature of additional investment needs, including regional and global investments that need to be considered. We consider changes in investment needs for known development interventions (e.g. providing more malaria bed nets to respond to the geographic expansion of malaria-affected areas as a result of climate change), new dedicated interventions for climate change adaptation, as well as regional and global goods. Available studies on the resource needs for climate change adaptation (e.g. World Bank (2010a), UNFCCC (2007), Parry et al. (2009), and Fankhauser (2010)) and our own analysis are used to obtain initial estimates for the incremental resources required to finance these interventions. Where important gaps exist in cost estimates, these are highlighted together with suggestions for how they can be closed. Finally, we consider the results to offer a definition for the 'additionality' of finance for climate change adaptation relative to development finance. 
In Chapter 4, we assess the interventions needed to achieve the SDG outcomes. We draw on UN Millennium Project (2004, 2005), UNTT (UNTT, 2013), UNCTAD (2014), and a large number of sector studies to identify the primary SDG investment areas and consider how cross-cutting investment needs can be addressed. Available needs assessments differ in methodologies, coverage, assumptions, and robustness, which makes them difficult to compare (UN Millennium Project, 2004; UNTT, 2013). We consider the relative merits of different methodologies and introduce a preliminary 'suitability score' to assess and compare the quality and suitability of the underlying analysis to inform the programming of public and private expenditure for the SDGs in low- and lower-middle-income countries. Based on a review of available needs assessments, we propose a unified framework for presenting operating and capital expenditure, additional investment needs owing to adaptation to climate change (drawing on the analysis in Chapter 3) and opportunities for private financing.

We then proceed to harmonize and aggregate investment needs across the SDG investment areas. Where possible, adjustments are made to fill gaps in available needs assessments, such as operating expenditure, missing interventions (e.g. non-communicable diseases in health needs assessments), or additional investment needs for climate change adaptation and mitigation, which no needs assessment integrates at present. Where two needs assessments from different SDG investment areas cover the same interventions, these overlaps are removed. We also consider to what extent investment needs (as opposed to outcomes) in one area are affected by the level of investments made in other areas. To the extent possible, such synergies and tradeoffs are quantified and considered in the aggregation of SDG investment needs.

The review in Chapter 4 demonstrates that only the health sector has developed a detailed, peer-reviewed literature on investment needs. In other SDG investment areas, available analyses are sparse and tend to be available only in the non-peer-reviewed 'grey literature'. Many have methodological and data shortcomings that are likely to adversely affect the nature and robustness of results obtained. The analysis conducted in the context of this thesis is therefore synthetic in nature and serves to highlight weaknesses in available assessments without resolving them directly.

To develop the analytical framework for the Global Fund in Chapter 5, we first identify the institution's key design principles that set it apart from other multilateral financing mechanisms (except Gavi), as well as major bilateral mechanisms, such as PEPFAR of the President's Malaria Initiative (PMI). Based on a systematic review of the scientific and policy literature on the Global Fund including public pronouncements by policymakers, we determine the five main criticisms leveled against the concept and design of the Global Fund at its inception in 2001. Using operational data from the Global Fund and drawing on independent assessments (e.g. Macro International (2009), HLIRP (2011), and DFID (2016)), we then assess empirically how the Global Fund's performance fared against each criticism and to what extent better-than-expected performance can be explained by the key design principles. Drawing on assessments of sector investment needs for sustainable development in Chapters 3 and 4, we consider lessons from the experience of the Global Fund for other SDG investment priorities.

Finally, in Chapter 6 we generate a new dataset combining information on funding proposals submitted to the Technical Review Panel (TRP) provided by the Global Fund secretariat; publicly available TRP recommendations and subsequent Board decisions; grants and their performance; and country characteristics consistent with predictors used in previous studies (Lu et al., 2006; Katz et al., 2010; Radelet and Siddiqi, 2007; Fan et al., 2013; Bowser et al., 2014). All proposals recommended for funding by the TRP and approved by the Global Fund Board were manually matched with signed grants from the Global Fund's grants dataset using proposal characteristics 
reported in both datasets (e.g. proposal name, disease component, country, funding volumes, and the year of submission).

We applied four sets of regression models to this dataset using ordinary-least squares (OLS) and ordered logistic models to determine the predictors for the following dependent variables: (i) natural log of total per capita funding requested by disease for each Global Fund round (OLS), (ii) TRP ratings of each proposal (ordered logistic), (iii) natural log of total per capita funding received by disease during the rounds-based funding mechanism (OLS), and (iv) average Phase 1 grant performance rating (ordered logistic). Consistent with the literature (Radelet and Siddiqi, 2007; Fan et al., 2013), each regression model was run separately for three disease categories (HIVIAIDS, including combined TB-HIV proposals, TB, and malaria) yielding a total of 12 panel data regressions. Each regression underwent standard statistical tests for outliers in the data, multicollinearity, normality of residuals, homoscedasticity, linearity, and redundancy of predictors, as recommended by Chatterjee and Hadi (2015).

\subsection{Structure of the thesis}

As indicated to some extent above, the thesis is therefore structured as follows: Chapter 2 introduces the SDG Index and Dashboards as analytical tools for assessing countries' SDG baselines and discusses findings. It illustrates the analytical value of the index by examining its relationship with other widely-used development indices and by showing how it accounts for cross-national differences in subjective well-being. The chapter also identifies data gaps that must be closed for SDG monitoring. Chapter 3 describes how development interventions and climate change adaptation can be integrated in the case of the MDGs in Africa and estimates associated resource needs. It is followed in Chapter 4 by an assessment of domestic and international financing needs for the SDGs by public and private sources. Chapter 5 assesses the overall performance of the Global Fund considering the institution's design principles and explores implications for the financing of other SDG priorities. In Chapter 6 we investigate the funding recommendations made by the Global Fund's TRP and consider implications for other SDG financing mechanisms. Chapter 7 summarizes the answers to the research questions, evaluates the methodologies and data, outlines future research priorities, and presents policy implications. 


\section{On metrics and financing for the Sustainable Development Goals}

This chapter is based on the paper: Schmidt-Traub G., C. Kroll, K. Teksoz, D. DurandDelacre, J.D. Sachs. 2017, "National baselines for the Sustainable Development Goals assessed in the SDG Index and Dashboards," Nature Geoscience 10: 547-55. 


\section{Chapter 2}

\section{National baselines for the}

Sustainable Development Goals assessed in the SDG Index and

\section{Dashboards}


On metrics and financing for the Sustainable Development Goals

This chapter introduces the first Sustainable Development Goal (SDG) Index and Dashboards as tools for assessing countries' baselines for the internationally agreed goals. The SDG Index and Dashboards synthesize available country-level data for all SDGs, providing quantitative measures for each goal to estimate the distance that must be closed to achieve the SDGs. They will be revised and updated annually. The 2016 SDG Index shows substantial variation across 149 countries for which data are available. All countries face major challenges in achieving the SDGs, and many countries' development strategies are imbalanced across the economic, social, and environmental SDG priorities. We illustrate the analytical value of the index for future research by examining its relationship with other widely used development indices and by showing how it accounts for cross-national differences in subjective well-being beyond conventional economic and social indicators. Given significant data gaps, scope and coverage of the SDG Index and Dashboards are limited, but we suggest that these analyses represent a starting point for a comprehensive assessment of national SDG baselines and can help policymakers determine priorities for early action and monitor progress. The tools also identify data gaps that must be closed for SDG monitoring. The chapter highlights major gaps in available data to track the SDGs and outlines how these can be filled. We close by outlining some implications for research and policy stemming from the SDG Index and Dashboards. 


\subsection{Introduction}

The Sustainable Development Goals (SDGs), agreed in 2015 by all 193 member states of the United Nations and complemented by commitments made in the Paris Climate Agreement, map out a broad spectrum of economic, social, and environmental objectives to be achieved by 2030 . Reaching these goals will require deep transformations in every country, as well as major efforts in monitoring and measuring progress. Here we introduce an SDG Index and Dashboards as analytical tools for assessing countries' baselines for the SDGs that can be applied by researchers in the cross-disciplinary analyses required for SDG implementation. The Index and Dashboards synthesize available country-level data for all $17 \mathrm{goals}$, and for each country estimate the size of the gap towards achieving the SDGs. They will be updated annually. All 149 countries for which sufficient data are available face significant challenges in achieving the goals, and many countries' development strategies are imbalanced across the economic, social, and environmental SDG priorities. We illustrate the analytical value of the index by examining its relationship with other widely-used development indices and by showing how it accounts for cross-national differences in subjective well-being. Given significant data gaps, scope and coverage of the SDG Index and Dashboards are limited, but we suggest that these analyses represent a starting point for a comprehensive assessment of national SDG baselines and can help policymakers determine priorities for early action and monitor progress. The tools also identify data gaps that must be closed for SDG monitoring.

To achieve the SDGs (United Nations, 2015a) (Table 6) and implement the Paris Climate Agreement, developed and developing countries alike will need to transform their energy systems, ecosystem management, agriculture and land use, urban management, material use, gender outcomes, health, education, governance, and other areas (Allen et al., 2016; van Vuuren et al., 2015). In addition to requiring greater financial resources and political commitments, these transformations will also place major demands on science to devise data and monitoring frameworks (Lu et al., 2015), to relate planetary boundaries to national sustainability objectives (Steffen et al., 2015; Häyhä et al., 2016), to develop innovative solutions, and to chart out integrated pathways for achieving the goals (Allen et al., 2016; Sachs et al., 2016c) taking account of the tradeoffs and synergies across goals and targets (Nilsson et al., 2016; ICSU and ISSC, 2015).

The predecessors to the SDGs, the Millennium Development Goals (MDGs) that expired in 2015, mobilized attention on addressing the challenges of extreme poverty, hunger, illiteracy and disease (McArthur, 2013). The MDGs helped spur advances on many fronts. In health, the MDGs have been associated with a significant acceleration of progress in some of the poorest countries (You et al., 2015; Kassebaum et al., 2014; McArthur and Rasmussen, 2017), which stands in contrast to the lack of progress on environmental sustainability observed under the three Rio conventions (Tollefson and Gilbert, 2012) and other MDG priorities, such as access to water supply (McArthur and Rasmussen, 2017).

The MDG experience suggests that global goals can serve as a management tool and report card that focus attention on complex sustainable development outcomes (McArthur, 2013) and that they can accelerate progress towards these outcomes. Yet success is far from guaranteed. Inter alia, it will require educating decision makers and the public in sustainable development; mobilizing science for diagnosing challenges, identifying solutions, developing long-term pathways, and tracking progress; mobilizing governments, business, and civil society for action around shared goals; and cooperation across countries to address planetary boundaries (Steffen et al., 2015) and 
other areas requiring international collaboration, such as implementing the Paris Climate Agreement or aid-financed investments in developing countries.

Compared with the eight MDGs, which were extracted from the Millennium Declaration by a team of officials working under UN Secretary-General Kofi Annan (McArthur, 2013), the SDGs represent a political compromise negotiated by the 193 member states of the United Nations that has been critically reviewed (ICSU and ISSC, 2015). The goals combine policy ends, such as ending extreme poverty or ending preventable child deaths, with means, such as development finance and maintaining a global partnership for development. Many SDGs focus on flows instead of focusing on stocks, as recommended by many scientists (Dasgupta and Mäler, 2000; Arrow et al., 2012; Stiglitz et al., 2009) since the report of the Brundtland Commission (Brundtland et al., 1987). Finally, the goals do not propose a hierarchy among the 17 goals and associated targets. In this chapter, we focus on how baselines for the SDGs can be established without aiming to resolve the criticisms of their design.

Good data and clear metrics are critical for each country to take stock of where it stands, devise pathways for achieving the goals, and track progress. The UN Statistical Commission has recommended a first set of 230 indicators to measure achievement of the SDGs, but many suggested indicators lack comprehensive, cross-country data and some even lack agreed statistical definitions (IAEG-SDGs, 2016a). More and better data are needed, but it will take years to build the necessary statistical systems, even if adequate resources were mobilized, which is currently not the case (United Nations, 2016a). Some governments have begun voluntary national reviews of progress on the SDGs, but they use indicators that are not harmonized internationally and lack comparability (United Nations, 2016b).

In the meantime, scientifically robust tools are needed to help operationalize the SDGs at the global, regional, national, and sub-national levels to begin a process of datadriven and evidence-based implementation and follow-up. Specifically, such tools should address the following questions: (i) What are the 2015 baselines for key SDG indicators at global, regional, national, and sub-national levels? (ii) How far is a country from achieving a SDG, and which are the country's most important SDG challenges? (iii) How can countries' overall progress towards implementing Agenda 2030 and the 17 SDGs be assessed, compared, and tracked over time? (iv) Which data gaps need to be filled most urgently to support better monitoring and facilitate peer-learning between countries regarding policies that help achieve the SDGs?

Reporting a large number of indicators alone, whilst providing much detail about specific domains, eventually leaves open the question of how to measure the aggregate performance of a country. Composite indices have well-known weaknesses (OECD and JRC, 2016), but they can synthesize complex information into a single number and may be more effective in stimulating public debates than a large number of individual scores which could result in 'cherry picking' (Dasgupta and Mäler, 2000; United Nations, 2016a). To inform policies for the achievement of complex integrated goals, a combination of composite measures and dashboards are therefore needed for the SDGs. Prominent examples of such composite indices in recent years include the Global Burden of Disease index for the health SDGs (GBD, 2016), the Environmental Performance Index (Hsu et al., 2016), the Ocean Health Index (Halpern et al., 2012), the Human Development Index (UNDP, 2015), the PISA education assessment (OECD, 2012), the Global Competitiveness Index (Schwab and Sala-i-Martín, 2016), the Global Peace Index (IEP, 2016), the Index of Economic Freedom (Miller, 2015), and many others. Some of these composite indices have had significant impact in drawing political attention (Waldow, 2009; Kroll, 2011), guiding policies, fostering learning across countries, and promoting more research (Dervis and Klugman, 2011; 
Wiseman, 2013). Yet, only the health-related SDG Index by the Global Burden of Disease collaboration, was specifically designed to track a part of the SDG agenda.

In order to assist countries in measuring their SDG baselines and to measure future progress, the Bertelsmann Stiftung and the Sustainable Development Solutions Network (SDSN) jointly released the first SDG Index and Dashboards in July 2016, with data covering 149 of 193 UN member states (Sachs et al., 2016b). The SDG Index and Dashboards build on an SDG Index published in 2015 by the Bertelsmann Stiftung with the support of SDSN, covering the 34 developed countries that were members of the Organization for Economic Cooperation and Development (OECD) (Kroll, 2015). The index and dashboards will be updated and revised annually. In this chapter, we present the SDG Index and Dashboards, explore differences in countries' performance, consider the empirical relationship to subjective well-being (SWB), and discuss how remaining gaps in data and analysis can be filled.

\subsection{The SDG Index and Dashboards}

The annual SDG Index provides a standardized, quantitative, transparent, and scalable composite measure of SDG baselines for 149 countries with sufficient data across the goals. It synthesizes 63 global indicators plus 14 additional indicators for OECD countries into an overall assessment of SDG baselines and ranks countries according to their starting points on the 17 SDGs. We included official SDG indicators (IAEGSDGs, 2016a) with data available for at least $80 \%$ of countries with a population greater than 1 million. Indicator gaps were filled using published data from other sources (Table 8). The authors consulted widely with expert communities on suitable indicators, including through a public consultation hosted by the SDSN. The methodology draws on Booysen (2002), GBD (2016), and OECD and JRC (2016). The details of the index are summarized in the methods summary and detailed in the Annex 2, which references all data sources and provides sensitivity analyses showing that the rankings are robust regarding alternative specifications.

Table 1 shows the SDG Index ranking for a selection of countries. Sweden is ranked first, having covered $84.5 \%$ of the distance towards the optimum outcome across SDG metrics for which data were included in the index. The annual SDG Dashboards (Figure 1) present baseline information by SDG to identify implementation priorities for each country 
On metrics and financing for the Sustainable Development Goals

Table 1 | SDG Index 2016: score and ranking

SDG Index ranking and scores (0 to 100) for the top ten countries, bottom five countries, members of the $G 20$, and other countries with population greater than 100 million. Rankings are out of 149 countries included in the 2016 SDG Index. Data for all countries are provided in Table 7.

\begin{tabular}{|r|l|r|}
\hline Rank & Country & Score \\
\hline 1 & Sweden & 84.5 \\
\hline 2 & Denmark & 83.9 \\
\hline 3 & Norway & 82.3 \\
\hline 4 & Finland & 81.0 \\
\hline 5 & Switzerland & 80.9 \\
\hline 6 & Germany & 80.5 \\
\hline 7 & Austria & 79.1 \\
\hline 8 & Netherlands & 78.9 \\
\hline 9 & Iceland & 78.4 \\
\hline 10 & United Kingdom & 78.1 \\
\hline 11 & France & 77.9 \\
\hline 13 & Canada & 76.8 \\
\hline 20 & Australia & 74.5 \\
\hline 25 & United States & 72.7 \\
\hline 27 & South Korea & 72.7 \\
\hline 35 & Italy & 70.9 \\
\hline 43 & Argentina & 66.8 \\
\hline 47 & Russia & 66.4 \\
\hline & & \\
\hline
\end{tabular}

\begin{tabular}{|r|l|r|}
\hline Rank & Country & Score \\
\hline 48 & Turkey & 66.1 \\
\hline 52 & Brazil & 64.4 \\
\hline 56 & Mexico & 63.4 \\
\hline 76 & China & 59.1 \\
\hline 85 & Saudi Arabia & 58.0 \\
\hline 95 & Philippines & 55.5 \\
\hline 98 & Indonesia & 54.4 \\
\hline 99 & South Africa & 53.8 \\
\hline 110 & India & 48.4 \\
\hline 115 & Pakistan & 45.7 \\
\hline 118 & Bangladesh & 44.4 \\
\hline 141 & Nigeria & 36.1 \\
\hline 145 & Chad & 31.8 \\
\hline 146 & Niger & 31.4 \\
\hline 147 & Congo, Dem. Rep. & 31.3 \\
\hline 148 & Liberia & 30.5 \\
\hline 149 & Central African & 26.1 \\
\hline & Republic & \\
\hline & & \\
\hline
\end{tabular}


Figure 1| SDG Dashboards 2016

SDG Dashboard for members of the G20 and other countries with population greater than 100 million. Dashboards for OECD countries calculated using an augmented set of 77 indicators, compared to $63 \mathrm{global}$ indicators for non-OECD countries. 'Green' signifies that the country has achieved the goal, 'yellow' points to significant challenges that remain, and 'red' warns that major challenges must be overcome to meet the goal. 'Grey' indicates an SDG for which there is no data.

\section{Non-OECD Countries}

\begin{tabular}{|c|c|c|c|c|c|c|c|c|c|c|c|c|c|c|c|c|c|c|}
\hline 10 & 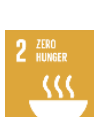 & 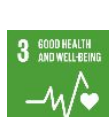 & 4 i guming & $\stackrel{5}{\ominus}$ & 6 & 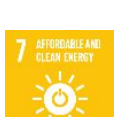 & 8 & 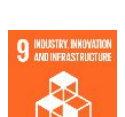 & 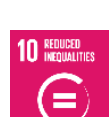 & 11 : & 12 sesesent & $133^{\mathrm{c}}$ & & 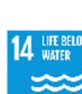 & & 5 & 16 & 17 (1) \\
\hline
\end{tabular}

\section{Argentina}

Bangladesh

Brazil

China

India

Indonesia

Nigeria

Pakistan

Philippines

Russia

Saudi Arabia
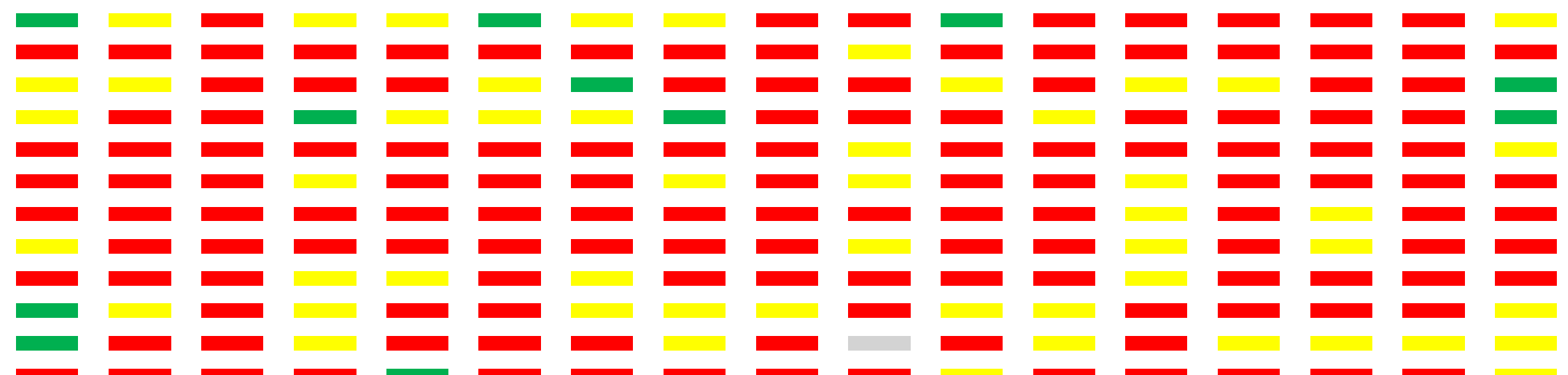

South Africa

s (Augmented SDG Dashboard)

OECD Coun

Canada

France

Germany

Italy

Japan

Mexico

South Korea

Turkey

UK

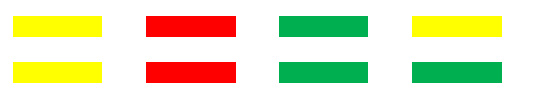

$(2$

$+1$

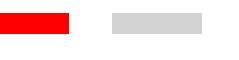

(n)

एक

USA

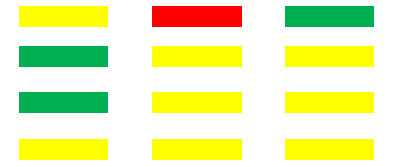

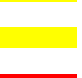

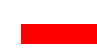

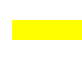

$\square$

(

-

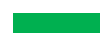

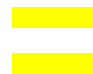

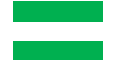

$\left[\begin{array}{ll}2 \\ \square\end{array}\right.$

$+2$

$\square \square$

$\square$

$\square$

$+$

ras

r

$\square$

\section{$\square$}

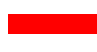

$\square$

$\square$

ए

-

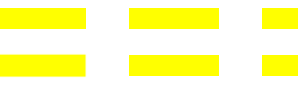

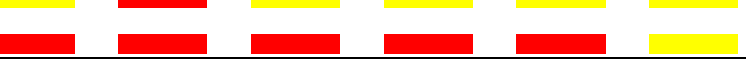

\section{$\square$}

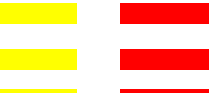




\subsection{Interpreting and applying the SDG Index and Dashboards}

The SDG Dashboards show that even the wealthiest countries face major challenges in meeting several of the SDGs, confirming the universal relevance of the goals and the need to consider the full SDG agenda to avoid 'cherry picking'. To meet the SDGs poor countries must make substantial progress in ending extreme poverty, ensuring access to essential infrastructure, curbing environmental degradation, and promoting social inclusion. Richer countries face fewer but nonetheless major challenges in addressing climate change, lowering inequalities, halting the loss of biodiversity, and contributing their fair share towards the global partnership to achieve the SDGs.

The SDG Index is correlated with the most widely used indicator of economic progress, per capita gross domestic product (GDP); the Human Development Index (HDI) that measures health, education, and income outcomes; the Global Competitiveness Index a common measure of countries' economic competitiveness; the Index of Economic Freedom, which estimates countries' governance; the Environmental Performance Index, which comprises a broad set of environmental indicators; and the Global Peace Index, as a broad measure of peace and conflict (Figure 2). The correlation is strongest with the HDI and GDP and weakest with the Index of Economic Freedom and the Global Peace Index.

However, substantial differences exist in performance within regions and across regions and income groups (Table 2). For example, average SDG Index scores for East and South Asia are lower than estimated from the global bi-variate relationships except for the Environmental Performance Index, suggesting that these countries have prioritized economic and social development over the environment. The reverse is true in countries in Eastern Europe and Central Asia, and Latin America and the Caribbean that perform better on the Environmental Performance Index than other regions. SubSaharan Africa performs worse on the SDG Index than on all other indices, except the $\mathrm{HDI}$. This finding is consistent with significant investments in health and basic education under the MDGs that have yet to be matched by similar investments in other SDG priorities. This illustrates the usefulness of a broader SDG Index in identifying imbalances in countries' development needs.

OECD members and high-income countries score better on the SDG Index than on the other indices suggesting that their development model is more balanced on average. However, as illustrated in the SDG Dashboards, OECD and high-income countries perform poorly on some goals, such as climate change, showing the limitations of only considering the aggregate SDG Index scores. 
Figure 2 | Correlation of SDG Index with other common development indices

Pairwise, population-weighted correlation of SDG Index scores by geographic region and income group with a, natural logarithm of GDP per capita (PPP) (IMF, 2016). b, Human Development Index (UNDP, 2015). c, Global Competitiveness Index (Schwab and Sala-i-Martín, 2016). d, Index of Economic Freedom (Miller, 2015). e, Environmental Performance Index (Hsu et al., 2016). f, Global Peace Index (IEP, 2016). Countries above trend line perform better on the SDG Index than suggested by correlations. Geographic regions: East and South Asia, Eastern Europe and Central Asia, Middle-East and North Africa, OECD countries, Latin America and Caribbean, Sub-Saharan Africa. Income groups: • high-income countries, - upper-middle-income countries, - lower-middle-income countries, and $\mathbf{\Delta}$ low-income countries.

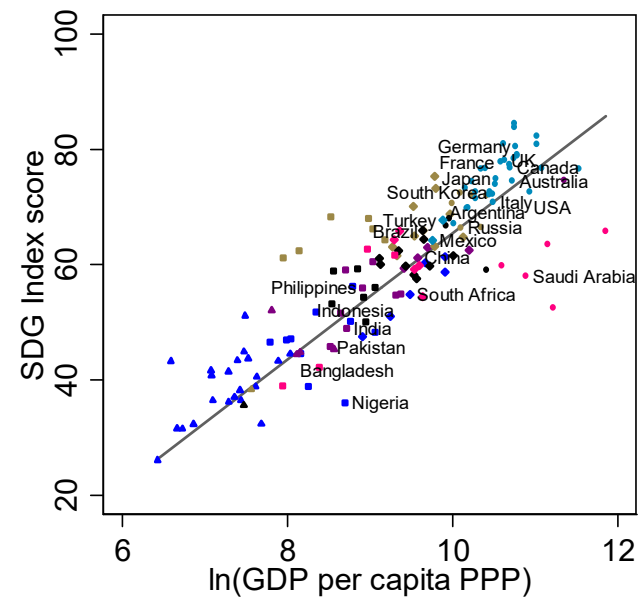

(a)

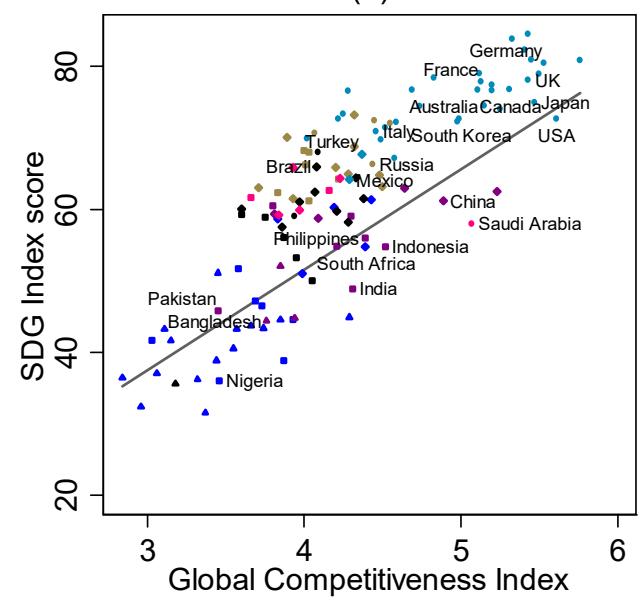

(c)

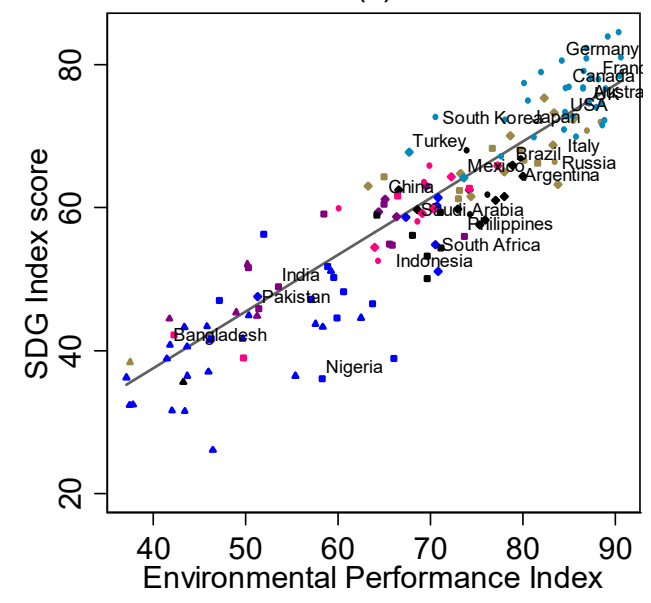

(e)

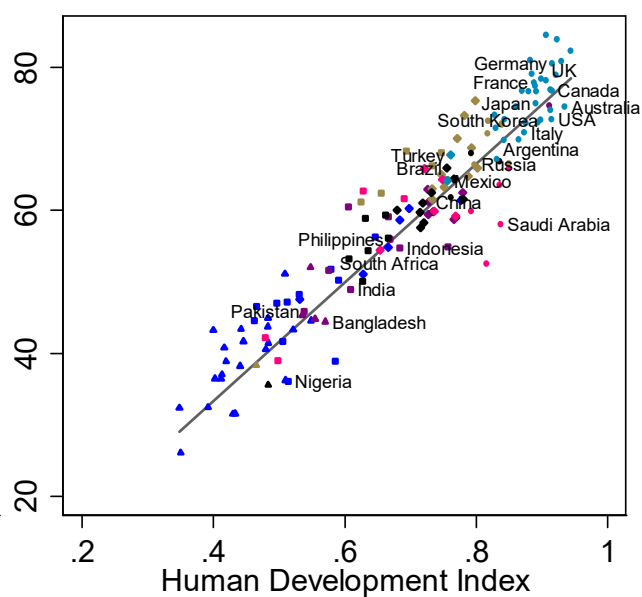

(b)

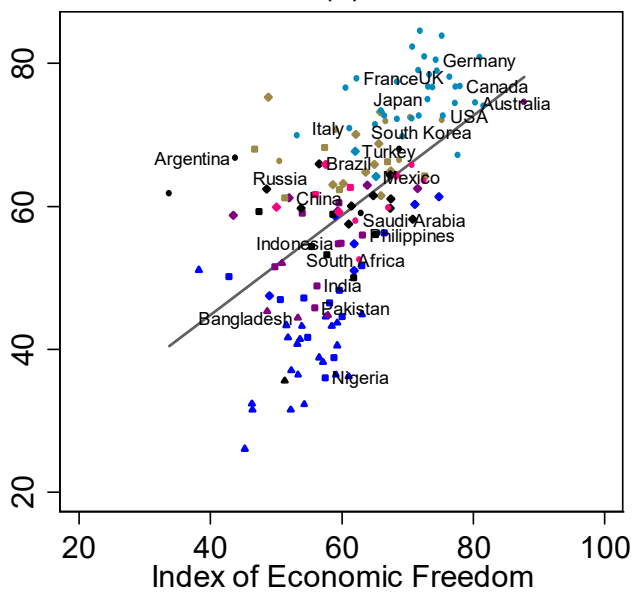

(d)

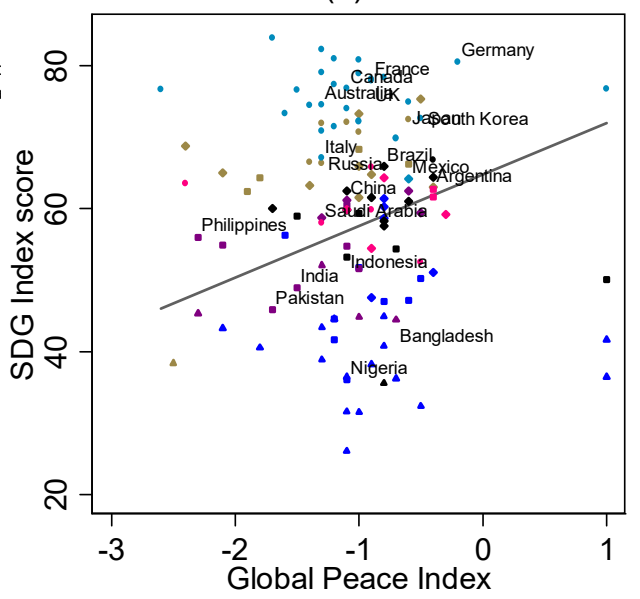

(f) 
Table 3 therefore disaggregates SDG Index scores by individual SDGs to identify which dimensions of the index drive each country's performance relative to others. Results show that different countries pursue different development models. For example, the United States ranks 9th in per capita GDP (IMF, 2016), but 25th in the SDG Index. Relative to its overall SDG score, the country experiences major deficits in inequality and peace and justice (SDGs 10 and 16), environmental objectives (SDGs 1215), and partnership for the Goals (SDG 17). Findings are similar for China, Russia, and the UK. Meanwhile, continental European countries tend to be more equal, but face major challenges on the environment goals and in some instances on economic performance. Some countries, notably from the Middle-East and North Africa, perform well on meeting basic needs, as measured by the HDI, but fall short on the SDG Index. For example, Saudi Arabia ranks 35th in the HDI but 85th (both out of 149) in the SDG Index owing to deficiencies in gender and income inequality and the environment. This shows how a combination of the SDG Index and the SDG Dashboards provide a richer understanding of a country's development challenges.

The Annex (Table 15) reports the SDG Index and its components for each country. These data can help countries identify when their development is by benchmarking performance across individual goals with average country performance as well as performance of countries at a similar stage of development.

The SDG Index is also partially correlated with subjective well-being in the presence of per capita GDP and unemployment, common correlates considered in the literature (Box 1). This illustrates the usefulness of the SDG Index in understanding determinants of and cross-country differences in SWB and other policy objectives. 
Table 2 | Relative performance on SDG-Index by region and income group

Table lists average distance of countries in each region from the estimated population-weighted relationship between the SDG Index and natural log of GDP per capita in Purchasing Power Parity, the Human Development Index, the Global Competitiveness Index, the Index of Economic Freedom, the Environmental Performance Index, and the Global Peace Index (Figure 2). All averages are weighted by countries' populations. Negative values suggest that countries in the region are on average below the trend line, i.e. their SDG Index score is lower than would be expected from the respective bivariate relationship.

\begin{tabular}{|c|c|c|c|c|c|c|}
\hline & $\begin{array}{l}\text { In(GDP per } \\
\text { capita } \\
\text { PPP) }\end{array}$ & $\begin{array}{c}\text { Human } \\
\text { Development Index }\end{array}$ & $\begin{array}{l}\text { Global Competitiveness } \\
\text { Index }\end{array}$ & $\begin{array}{l}\text { Index of } \\
\text { Economic } \\
\text { Freedom }\end{array}$ & $\begin{array}{l}\text { Environmental } \\
\text { Performance Index }\end{array}$ & $\begin{array}{l}\text { Global } \\
\text { Peace } \\
\text { Index }\end{array}$ \\
\hline \multicolumn{7}{|l|}{ By region: } \\
\hline East and South Asia & -1.10 & -0.75 & -4.11 & -0.79 & 2.19 & -6.71 \\
\hline $\begin{array}{l}\text { Eastern Europe and } \\
\text { Central Asia }\end{array}$ & 4.10 & 2.13 & 10.83 & 12.38 & -1.87 & 6.54 \\
\hline $\begin{array}{l}\text { Latin America and the } \\
\text { Caribbean }\end{array}$ & 2.18 & 0.63 & 10.11 & 5.85 & -2.71 & 2.64 \\
\hline $\begin{array}{l}\text { Middle-East and North } \\
\text { Africa }\end{array}$ & -2.31 & 0.51 & 7.80 & 3.67 & 1.48 & 1.66 \\
\hline Sub-Saharan Africa & -0.91 & 0.45 & -6.08 & -14.00 & -5.72 & -20.06 \\
\hline OECD members & 2.66 & 0.98 & 8.42 & 6.71 & 4.17 & 14.95 \\
\hline \multicolumn{7}{|l|}{ By income group: } \\
\hline Low-income countries & 2.66 & 1.15 & -5.74 & -11.79 & -0.33 & -18.33 \\
\hline $\begin{array}{l}\text { Lower-middle-income } \\
\text { countries }\end{array}$ & -1.55 & -0.44 & -3.79 & -6.04 & -0.52 & -10.82 \\
\hline $\begin{array}{l}\text { Upper-middle-income } \\
\text { countries }\end{array}$ & -0.28 & -0.08 & 0.71 & 5.66 & 1.93 & 0.77 \\
\hline High-income countries & 1.81 & 0.28 & 8.69 & 8.55 & 2.93 & 13.87 \\
\hline
\end{tabular}


Table 3 | Deviation from average country SDG Index score by SDG

Difference between overall SDG Index score (Table 1) and scores for each SDG for G20 members and countries with a population greater than 100 million.

\begin{tabular}{|c|c|c|c|c|c|c|c|c|c|c|c|c|c|c|c|c|c|}
\hline Country & $\begin{array}{c}\text { SDG } \\
1\end{array}$ & $\begin{array}{c}\text { SDG } \\
2\end{array}$ & $\begin{array}{c}\text { SDG } \\
3\end{array}$ & $\begin{array}{c}\text { SDG } \\
4\end{array}$ & $\begin{array}{l}\text { SDG } \\
5\end{array}$ & $\begin{array}{c}\text { SDG } \\
6\end{array}$ & $\begin{array}{c}\text { SDG } \\
7\end{array}$ & $\begin{array}{c}\text { SDG } \\
8\end{array}$ & $\begin{array}{c}\text { SDG } \\
9\end{array}$ & $\begin{array}{c}\text { SDG } \\
10\end{array}$ & $\begin{array}{c}\text { SDG } \\
11\end{array}$ & $\begin{array}{c}\text { SDG } \\
12\end{array}$ & $\begin{array}{c}\text { SDG } \\
13\end{array}$ & $\begin{array}{c}\text { SDG } \\
14\end{array}$ & $\begin{array}{c}\text { SDG } \\
15\end{array}$ & $\begin{array}{c}\text { SDG } \\
16\end{array}$ & $\begin{array}{c}\text { SDG } \\
17\end{array}$ \\
\hline Argentina & 33.2 & 9.3 & 9.3 & 24.5 & 8.0 & 30.8 & 19.7 & -3.8 & -35.1 & -17.5 & 22.9 & -21.1 & 15.7 & -27.8 & -21.5 & -24.1 & -22.6 \\
\hline Australia & 25.5 & -5.1 & 11.7 & 21.3 & 3.1 & 25.2 & 9.8 & 4.7 & -0.2 & 6.3 & 13.2 & 1.9 & -33.8 & -24.5 & -23.9 & -2.2 & -33.1 \\
\hline Bangladesh & -7.9 & -6.1 & 6.3 & 9.9 & 6.9 & 31.5 & -1.2 & 10.5 & -38.6 & 37.7 & -30.5 & 2.8 & 20.5 & -1.6 & 0.2 & 1.3 & -64.0 \\
\hline Brazil & 27.0 & 7.9 & 2.7 & 8.7 & 1.6 & 26.2 & 24.8 & -1.7 & -28.6 & -43.6 & 15.8 & -18.9 & 20.9 & -11.8 & -8.2 & -31.4 & -15.4 \\
\hline Canada & 23.2 & 1.8 & 7.5 & 8.0 & 3.3 & 22.8 & 14.1 & 8.4 & -13.1 & 4.1 & 10.6 & -7.3 & -14.6 & -20.9 & -19.2 & 2.3 & -31.0 \\
\hline China & 22.6 & 6.7 & 12.1 & 19.8 & 9.9 & 25.1 & 14.8 & 8.8 & -15.7 & -5.4 & -18.0 & -10.4 & -22.0 & -29.2 & -12.5 & -4.5 & -35.8 \\
\hline France & 22.1 & -3.2 & 6.9 & 6.5 & 0.9 & 20.2 & 15.4 & -10.3 & -8.4 & 8.2 & 7.6 & -2.8 & 5.7 & -22.7 & -17.5 & -6.6 & -22.0 \\
\hline Germany & 19.5 & 6.3 & 6.8 & 4.6 & -1.3 & 17.3 & 6.9 & -3.8 & -7.6 & 11.7 & 3.6 & -1.5 & -5.8 & -28.7 & -7.9 & -2.4 & -17.7 \\
\hline India & 20.2 & -18.5 & 3.1 & 12.5 & -18.5 & 21.2 & 9.1 & 10.2 & -27.1 & 28.5 & -21.8 & 4.4 & 14.2 & -9.7 & -13.1 & 9.7 & -32.8 \\
\hline Indonesia & 22.1 & -9.9 & -1.0 & 19.1 & 7.3 & 21.3 & 7.0 & 9.0 & -33.2 & 11.4 & -6.0 & -8.3 & 29.2 & -12.0 & -21.1 & 4.8 & -45.8 \\
\hline Italy & 29.1 & -3.2 & 13.4 & 16.5 & -3.9 & 26.4 & 16.9 & -10.4 & -19.7 & 0.9 & 10.1 & 5.1 & 7.8 & -28.5 & -6.7 & -15.9 & -37.8 \\
\hline Japan & 25.0 & 0.4 & 10.3 & 9.0 & -15.3 & 23.4 & 12.7 & 1.6 & 12.6 & 7.2 & 7.8 & -4.1 & -6.4 & -32.3 & -21.4 & 3.1 & -33.6 \\
\hline Mexico & 31.9 & -0.6 & 9.1 & 14.0 & 9.0 & 26.0 & 17.1 & -1.4 & -35.3 & -21.9 & 21.3 & -5.8 & 21.3 & -9.8 & -27.6 & -24.6 & -35.7 \\
\hline Nigeria & -13.9 & 7.5 & -16.9 & -16.3 & -11.9 & 15.3 & 2.7 & 6.0 & -19.6 & 1.8 & -16.6 & 10.5 & 52.1 & -2.7 & 36.7 & 1.2 & -36.1 \\
\hline Pakistan & 27.0 & 7.9 & 2.7 & 8.7 & 1.6 & 26.2 & 24.8 & -1.7 & -28.6 & -43.6 & 15.8 & -18.9 & 20.9 & -11.8 & -8.2 & -31.4 & -43.0 \\
\hline Philippines & 24.9 & -9.8 & 1.8 & 10.3 & 6.9 & 27.0 & 11.4 & -0.5 & -38.8 & -2.6 & 13.3 & -9.1 & 27.4 & -2.4 & -21.0 & -3.4 & -42.7 \\
\hline $\begin{array}{l}\text { Russian } \\
\text { Federation }\end{array}$ & 33.6 & -11.3 & 9.0 & 16.8 & 1.3 & 20.9 & 18.9 & 13.2 & -21.9 & -5.4 & 15.7 & -13.1 & -0.6 & -12.1 & -6.7 & -29.2 & -29.2 \\
\hline Saudi Arabia & 42.0 & -3.6 & 14.4 & 30.8 & -18.7 & 6.6 & 24.5 & 14.6 & -7.4 & -58.0 & -58.0 & -4.7 & -3.9 & -6.7 & 4.4 & 5.6 & -39.9 \\
\hline South Africa & 21.1 & 5.9 & -12.9 & 17.9 & 26.6 & 25.7 & 19.7 & -25.8 & -12.3 & -54.8 & 26.0 & -8.4 & 11.2 & -10.8 & -20.1 & -13.0 & -12.3 \\
\hline South Korea & 27.3 & 6.0 & 8.4 & 14.2 & -10.0 & 22.0 & 16.1 & 17.5 & 10.3 & 13.2 & -3.2 & 8.8 & -6.3 & -42.2 & -27.2 & -15.2 & -39.7 \\
\hline Turkey & 31.9 & -2.9 & 6.5 & 13.4 & -25.1 & 28.7 & 20.0 & -3.9 & -24.9 & -6.6 & 14.5 & -8.6 & 16.5 & -21.2 & -15.9 & -11.6 & -36.6 \\
\hline $\begin{array}{l}\text { United } \\
\text { Kingdom }\end{array}$ & 21.9 & -0.1 & 6.6 & 9.3 & 3.2 & 20.9 & 8.9 & -2.0 & -5.6 & -6.2 & 10.7 & 4.7 & -1.7 & -28.4 & -31.6 & 0.5 & -11.1 \\
\hline United States & 27.3 & 15.7 & 7.5 & 2.4 & 1.7 & 25.5 & 14.9 & 9.8 & 9.1 & -13.6 & 15.7 & -11.2 & -18.7 & -27.5 & -28.4 & -11.8 & -18.4 \\
\hline
\end{tabular}




\section{Box 1 | Applying the SDG Index as a predictor of subjective well-being}

SWB is increasingly considered a key aggregate objective of public policy (Stiglitz et al., 2009; OECD, 2013). It is commonly measured using the Cantril Ladder, which asks survey respondents to rate their well-being on a scale from zero to ten with ten denoting maximum well-being (Helliwell et al., 2016b). To assess whether progress in achieving the 17 SDGs, as measured by the SDG Index, is likely to be associated with improvements in SWB, we investigated whether the SDG Index is partially correlated with SWB controlling for the two main macroeconomic correlates of SWB identified in the SWB literature: per capita income and unemployment (Clark and Oswald, 1994; Dolan et al., 2008; Delhey and Kroll, 2013).

As shown in Table 4 (column 1), the SDG Index is indeed partially correlated with SWB when controlling for GDP per capita and unemployment $(p=0.014)$. We also tested the partial correlation of SWB with three other widely used synthetic cross-country indexes: the $\mathrm{HDI}$, the Global Competitiveness Index, and the Index of Economic Freedom as an extension of a previous analysis that compared three prevalent theories of societal well-being (libertarianism, consumerism and holism) (Helliwell et al., 2016b). None of the three alternative indices is partially correlated with SWB at the 0.05 level when controlling for GDP per capita and unemployment (partial correlations 1-5). These results suggest that progress towards the SDGs may well portend a rise in SWB.

To investigate which indicators included in the SDG Index account for the partial correlation, we generated partial correlations for each indicator included in the index with SWB. Table 17 lists the indicators that exhibited a significant $(p<0.05)$ partial correlation with SWB in the presence of GDP per capita and unemployment. These preliminary results are consistent with the literature where health status and perceptions of corruption have been shown to play a role in determining SWB (Helliwell et al., 2016b; Dolan et al., 2008). They also identify potential regressors for SWB that have not been studied widely in the literature. We emphasize, however, that we have not yet demonstrated causation running from SDG progress to SWB. We intend to pursue these issues in our future research.

\section{Table 4 | Partial correlation coefficients with Cantril Ladder}

Partial correlations between SWB measured by the Cantril Ladder and the following explanatory variables: Sustainable Development Goals Index, Global Competitiveness Index, Human Development Index, Index of Economic Freedom, GDP per capita, and unemployment. PPP - Purchasing Power Parity. Significance of partial correlation coefficients is reported in parenthesis using the following convention: ${ }^{* * *} p<0.001,{ }^{* *} p<0.01,{ }^{*} p<0.05$; all $p$-values are one-sided. Summary statistics and data sources for all variables are provided in Table 16.

\begin{tabular}{|c|c|c|c|c|c|}
\hline & \multicolumn{5}{|c|}{$\begin{array}{c}\text { Partial Correlation Coefficients with SWB measured } \\
\text { by Cantril Ladder }\end{array}$} \\
\hline & (1) & (2) & (3) & (4) & (5) \\
\hline \multirow{2}{*}{$\begin{array}{l}\text { Sustainable Development } \\
\text { Goals Index }\end{array}$} & $0.227^{* *}$ & & & & $0.185^{*}$ \\
\hline & $(0.014)$ & & & & $(0.049)$ \\
\hline \multirow{2}{*}{$\begin{array}{l}\text { Global Competitiveness } \\
\text { Index }\end{array}$} & & 0.167 & & & 0.075 \\
\hline & & $(0.072)$ & & & $(0.431)$ \\
\hline \multirow[t]{2}{*}{ Human Development Index } & & & 0.123 & & -0.078 \\
\hline & & & $(0.188)$ & & $(0.407)$ \\
\hline \multirow[t]{2}{*}{ Index for Economic Freedom } & & & & 0.130 & 0.066 \\
\hline & & & & $(0.164)$ & $(0.483)$ \\
\hline \multirow[t]{2}{*}{ Log GDP per capita (PPP) } & $0.477^{\star \star \star}$ & $0.556^{\star * \star}$ & $0.323^{* * *}$ & $0.738^{\star \star *}$ & $0.328^{* * *}$ \\
\hline & $(0.000)$ & $(0.000)$ & $(0.000)$ & $(0.000)$ & $(0.000)$ \\
\hline \multirow[t]{2}{*}{ Unemployment } & $-0.405^{* * *}$ & $-0.317^{* * *}$ & $-0.405^{*}$ & $-0.391^{* * *}$ & $-0.335^{* * *}$ \\
\hline & $(0.000)$ & $(0.001)$ & $(0.000)$ & $(0.000)$ & $(0.000)$ \\
\hline Observations & 119 & 119 & 119 & 119 & 119 \\
\hline
\end{tabular}




\subsection{Major data gaps for the SDGs}

Three types of data challenges need to be addressed to improve the measurement of baselines for the SDGs and to ensure effective monitoring, as summarized in

Table 5. First, some SDG priorities lack scientifically robust indicator definitions that can be applied in a broad range of countries. Developing or improving such definitions will require a collaboration between the respective academic communities with statistical offices and policymakers. International organizations could convene such dialogues and support the production of handbooks on new measurement areas, such as the OECD Guidelines of Measuring Subjective Well-Being (OECD, 2013).

Second, some indicators require better and more frequent data collection and dissemination, particularly in poor countries and small-island economies where data are either unavailable or estimated too infrequently to allow for the reliable estimation of trends over time. In many instances this will require substantial additional investments in statistical systems and data collection mechanisms (Espey et al., 2015). Countries with small populations need to consider alternative methods for approximating some data since standard survey techniques may become inoperable.

Third, in other areas data are collected by scientists or available through big data but is not adequately used to inform official SDG monitoring efforts at national, regional, and global levels. This applies particularly to perception-based indicators (e.g. on corruption or subjective well-being) but also other metrics, which are well accepted scientifically, but not used by many national statistical offices and not included among the proposed official SDG indicators (IAEG-SDGs, 2016a). In some areas, such as food loss and waste, greater efforts are needed to bring SDG-relevant commercial data into the public domain and to improve the availability of such data.

Because of these data gaps some SDGs remain poorly measured. For example, we are unable to include indicators on gender-based violence. SDG 12 on sustainable consumption and production presents some of the greatest data challenges. This is true, for example, in the case of the Material Footprint Index (MFI) (Wiedmann et al., 2015) which we did not include in the SDG Index for two reasons. First, except for fossil fuels, which are covered under SDG 13, it is not clear how per capita consumption of specific materials (biomass, construction minerals, and metal ores) relates to local and global environmental impact. Second, the MFI aggregates consumption across a broad range of different materials on a per-kg basis even though one $\mathrm{kg}$ of biomass might have a different environmental impact than one $\mathrm{kg}$ of iron ore or building stone.

Another important shortcoming in the current SDG Index and available SDG data are the inadequate measurement of spill-over effects of one country's actions on the ability of other countries to achieve the SDGs, a phenomenon sometimes referred to as leakage. Spill-overs include greenhouse gas emissions, land degradation, endangerment of species, fisheries depletion, forced or child labor, groundwater depletion, financial secrecy, and other detrimental effects through global supply chains and international trade. Better addressing spill-over effects as well as sustainable consumption and production patterns in the SDG Index may significantly change the ranking of some highincome countries that for example consume large volumes of environmental resources or promote policies with negative impacts on other countries' SDG baselines. See Annex 2 for more details. 
Table 5 | Principal SDG data issues and gaps

Table summarizes SDG priorities requiring more or better data and outlines principal data issues and associated data collection methods.

\begin{tabular}{|c|c|c|c|}
\hline SDG & $\begin{array}{l}\text { Areas requiring } \\
\text { more or better } \\
\text { data }\end{array}$ & Principal data issues & $\begin{array}{l}\text { Principal data col- } \\
\text { lection methods }\end{array}$ \\
\hline 1 & Extreme poverty & $\begin{array}{l}\text { Data availability (frequency, timeliness, and international compara- } \\
\text { bility) }\end{array}$ & Household surveys \\
\hline 2 & $\begin{array}{l}\text { Agriculture and } \\
\text { food security }\end{array}$ & $\begin{array}{l}\text { Indicator concepts (land tenure, food loss and waste, comparable } \\
\text { yield gaps by agro-ecological zone, environmental sustainability of } \\
\text { key agricultural commodities and farming practices, livestock sys- } \\
\text { tems, and aquaculture); data availability (diets and major micronutri- } \\
\text { ent deficiencies, use efficiency of agricultural inputs); and use of } \\
\text { available data (e.g. published data on nitrogen use efficiency, com- } \\
\text { mercial data on fertilizer use, food loss and waste) }\end{array}$ & $\begin{array}{l}\text { Agricultural and } \\
\text { household surveys, } \\
\text { business data, re- } \\
\text { mote sensing }\end{array}$ \\
\hline 3 & Health & $\begin{array}{l}\text { Indicator concepts (affordability of healthcare and financial risks } \\
\text { from poor health, mental health metrics) }\end{array}$ & $\begin{array}{l}\text { Administrative data, } \\
\text { household surveys }\end{array}$ \\
\hline 4 & Education & $\begin{array}{l}\text { Indicator concepts (access and learning outcomes for early child- } \\
\text { hood development, primary and secondary school) }\end{array}$ & $\begin{array}{l}\text { Administrative data, } \\
\text { household surveys }\end{array}$ \\
\hline 5 & Gender & $\begin{array}{l}\text { Indicator concepts (economic empowerment of women), data availa- } \\
\text { bility (violence against women and its underreporting, gender dis- } \\
\text { aggregation of major surveys) and stratification across survey instru- } \\
\text { ments }\end{array}$ & $\begin{array}{l}\text { Surveys and ad- } \\
\text { ministrative data }\end{array}$ \\
\hline 6 & $\begin{array}{l}\text { Drinking water } \\
\text { quality and water } \\
\text { pollution }\end{array}$ & $\begin{array}{l}\text { Indicator concepts (effective access to water supply and sanitation), } \\
\text { data availability (quality of drinking water, surface water, and } \\
\text { groundwater) }\end{array}$ & $\begin{array}{l}\text { Household surveys, } \\
\text { administrative data, } \\
\text { physical sampling }\end{array}$ \\
\hline 7 & Clean energy & $\begin{array}{l}\text { Indicator concepts (leading indicators for energy transformation), } \\
\text { use of business data }\end{array}$ & $\begin{array}{l}\text { Administrative data, } \\
\text { business data }\end{array}$ \\
\hline 8 & Decent work & $\begin{array}{l}\text { Indicator concepts (internationally comparable decent work indica- } \\
\text { tors) }\end{array}$ & $\begin{array}{l}\text { Business surveys, } \\
\text { household surveys }\end{array}$ \\
\hline 9 & Infrastructure & $\begin{array}{l}\text { Indicator concepts (adequacy of transport, water, energy, and other } \\
\text { infrastructure) }\end{array}$ & Administrative data \\
\hline 10 & Inequality & $\begin{array}{l}\text { Indicator concepts (vertical mobility and equality of opportunity), } \\
\text { data availability (Gini) }\end{array}$ & $\begin{array}{l}\text { Household surveys, } \\
\text { administrative data }\end{array}$ \\
\hline 11 & $\begin{array}{l}\text { Transport and } \\
\text { waste manage- } \\
\text { ment }\end{array}$ & $\begin{array}{l}\text { Indicator concepts (access to and affordability of public and private } \\
\text { transport, categories of waste and their re-use), better use of scien- } \\
\text { tific data from trade statistics and input-output tables }\end{array}$ & $\begin{array}{l}\text { Administrative data, } \\
\text { surveys }\end{array}$ \\
\hline 12 & $\begin{array}{l}\text { Sustainable con- } \\
\text { sumption and } \\
\text { production pat- } \\
\text { terns }\end{array}$ & $\begin{array}{l}\text { Indicator concepts (to better track environmental impact of material } \\
\text { use and relationship to biophysical constraints, recycling and re- } \\
\text { use), data availability }\end{array}$ & $\begin{array}{l}\text { Administrative data, } \\
\text { trade statistics }\end{array}$ \\
\hline 13 & $\begin{array}{l}\text { Climate change } \\
\text { mitigation and } \\
\text { adaptation }\end{array}$ & $\begin{array}{l}\text { Indicator concepts (harmonized standards for emissions from for- } \\
\text { estry, carbon prices, trade impact on emissions, adaptation } \\
\text { measures), better use of business data (e.g. from insurance indus- } \\
\text { try) }\end{array}$ & $\begin{array}{l}\text { Administrative data, } \\
\text { remote sensing }\end{array}$ \\
\hline 14 & $\begin{array}{l}\text { Ocean ecosys- } \\
\text { tems }\end{array}$ & $\begin{array}{l}\text { Indicator concepts (sustainable fisheries, marine litter, marine pro- } \\
\text { tected areas, threatened species, attribution of changes in high seas } \\
\text { to countries), better integration of scientific data }\end{array}$ & $\begin{array}{l}\text { Surveys, direct } \\
\text { measurement and } \\
\text { remote sensing }\end{array}$ \\
\hline 15 & $\begin{array}{l}\text { Terrestrial eco- } \\
\text { systems }\end{array}$ & $\begin{array}{l}\text { Indicator concepts (protected areas, trade in endangered species, } \\
\text { biomes of global significance, leading indicators of ecosystem } \\
\text { health), data availability, better integration of scientific data }\end{array}$ & $\begin{array}{l}\text { Surveys, direct } \\
\text { measurement and } \\
\text { remote sensing }\end{array}$ \\
\hline 16 & $\begin{array}{l}\text { Modern slavery } \\
\text { and access to } \\
\text { justice }\end{array}$ & $\begin{array}{l}\text { Indicator concepts (human trafficking, modern slavery, access to } \\
\text { justice, financial secrecy), data availability }\end{array}$ & $\begin{array}{l}\text { Administrative data, } \\
\text { household surveys }\end{array}$ \\
\hline 17 & $\begin{array}{l}\text { International fi- } \\
\text { nance and trade } \\
\text { for the SDGs }\end{array}$ & $\begin{array}{l}\text { Indicator concepts (private and public non-concessional SDG fi- } \\
\text { nance, tax heavens) }\end{array}$ & Administrative data \\
\hline
\end{tabular}




\subsection{Implications for future research and policy}

The SDG Index provides a first comprehensive assessment of countries' starting points on the internationally-agreed SDGs. In contrast to GDP per capita and more narrowly defined indices, it addresses the full spectrum of economic, social, and environmental challenges that countries face in achieving the SDGs providing additional insights into countries' sustainable development needs. The SDG Dashboards show significant variation in starting points across countries, and they underscore that every country falls short on a number of SDG priorities. Both tools show that countries pursue different development strategies, and that many countries need to better balance economic, social, and environmental objectives. These questions require further scientific analyses in all major disciplines relating to the 17 SDGs.

The scope and coverage of the SDG Index is currently limited by the availability of data, and significant gaps exist, such as sustainable consumption or gender-based violence. Official statistics do not adequately cover the goals, so they will need to be complemented by robust, scientific data. Future research into improving the SDG Index will focus on adding more SDG metrics as data become available and relating national performance thresholds to be achieved by 2030 to planetary boundaries (Steffen et al., 2015; Häyhä et al., 2016).

To better reflect regional priorities, the SDG Index and Dashboards can in the future be augmented with variables that are of relevance in each region, such as malaria metrics in sub-Saharan Africa. Likewise, countries can apply SDG Indices at the subnational level to compare starting points across states and provinces. A first prototype SDG Index for Cities has been launched for US cities (Prakash et al., 2017a), and similar indices can be designed for cities around the world.

Finally, more countries need to be included in the Index. For example, compact versions of the SDG Index can be developed for small-island developing states that tend to lack data for key socioeconomic and environmental variables owing to their small population size and limited data collection capacities.

In view of current data limitations, the SDG Index and Dashboards do not provide a comprehensive measure of sustainable development. The likely addition of indicators in future editions of the SDG Index, combined with the fact that data for many variables are only updated infrequently and with different periodicities, limits the scope for calculating year-on-year changes in the SDG Index and Dashboards and for using them as monitoring tools (see also the Annex 2). When publishing updates to the SDG Index we will consider how progress towards the goals can be estimated using the SDG Index and Dashboards to help inform official SDG monitoring processes.

\subsection{Methods summary}

As described in the Annex 2, the SDG Index and Dashboards include the more than 230 official SDG indicators(IAEG-SDGs, 2016b) proposed by the Inter-Agency and Expert Group on SDG Indicators (IAEG-SDGs) that met tests of data availability and usability in a global index. Where official SDG indicators did not meet the criteria for data selection or where indicator gaps remained, we considered official and other metrics published in the peer-reviewed literature, as well as major databases and reports on development and environmental indicators. We also consulted with a broad range of experts and conducted a public consultation on an earlier draft of the analysis that generated 56 submissions. Since one purpose of the SDG Index and Dashboards is to highlight missing data we did not impute missing data except for four variables that 
lacked data for high-income or low-income countries (Annex 2). Each of the 17 SDGs has at least 1 (SDGs 1 and 11) and a maximum of 11 indicators. The raw data for the SDG Index are available for download with this thesis.

Data for each indicator was normalized on a linear scale of 0 to 100 . A score of 0 was defined by performance at the 2.5 percentile to ensure extreme values did not skew the distribution (OECD and JRC, 2016). A score of 100 denotes target achievement and 'leaving no one behind' (United Nations, 2015a) (e.g. zero extreme poverty, 100\% school completion). Some SDG target thresholds are below levels achieved today in high-performing countries. For example, the official 2030 SDG Target for child mortality is 25 per 1000 live births, while the top-performing countries have rates of 2.3-3.9 per 1000 . For such SDG indicators, the maximum score of 100 was set as an aspirational target surpassing the SDG Target (e.g. 0 child mortality per 1000 live births). In cases where no quantitative SDG target or an aspirational target could be identified we used the average of the top five performing countries as the benchmark for top performance. In this way, the SDG Index defined an optimum outcome across the 17 goals that countries, including advanced economies, should aim for by 2030.

We aggregated indicators arithmetically within each goal and then averaged across goals, applying the same weight to every goal according to Equation 1:

$$
I_{i}\left(N_{i}, N_{i j}, I_{i j k}\right)=\sum_{j=1}^{N_{i}} \frac{1}{N_{i}} \sum_{k=1}^{N_{i j}} \frac{1}{N_{i j}} I_{i j k}
$$

Where $\mathrm{I}_{\mathrm{i}}$ is the index score for country $\mathrm{i}, \mathrm{N}_{\mathrm{i}}$ the number of SDGs for which the country has data, $\mathrm{N}_{\mathrm{ij}}$ the number of indicators for SDG $\mathrm{j}$ for which data are available for country $\mathrm{i}$, and $\mathrm{I}_{\mathrm{ijk}}$ denotes the score of indicator $\mathrm{k}$ under SDG $\mathrm{j}$ for country $\mathrm{i}$. This weighting for the SDG Index is subjective, as is the case with all composite indices (OECD and JRC, 2016; Booysen, 2002). Our approach is consistent with the intention of UN member states who framed the SDGs as an "integrated and indivisible" agenda (United Nations, 2015a), whereby the goals have equal priority. Annex 2 discusses the motivation for and implications of alternative aggregation methodologies.

Our methodology differs in some aspects from the approach of the health-related SDG Index (GBD, 2016) produced by the Global Burden of Disease consortium. Its authors relied on extensive modelling and interpolation to generate data for 188 countries (Wang et al., 2016). Meanwhile, we refrained from modelling data due to the heterogeneity of the data sources used for the SDG Index, the absence of robust time series for some survey-based and other metrics, and the limited geographical coverage of many indicators for important SDG priorities. Moreover, the SDG Index seeks to draw attention to data gaps, so we limited the imputation of missing data to measures of extreme deprivation, which were not collected in rich countries and which we could confidently set at zero in the high-income countries (Annex 2 ).

For the health goals and several other SDGs, both the health-related SDG Index and the SDG Index define the upper bounds according to the average performance of the top countries. But this approach of relying on 'best performers' could not be used for greenhouse gas emission reductions, sustainable energy use, or other areas where no country currently meets sustainability thresholds. For such variables, absolute target values had to be defined (Annex 2). The OECD pilot assessment used a similar methodology to ours and applied absolute thresholds (OECD, 2016a), while Kroll (2015) scored OECD countries based on their relative performance. 
To construct the SDG Dashboards, each indicator was assigned absolute performance thresholds (Table 11). For each goal, a country's SDG Dashboard rating was determined by the rating of the worst-performing indicator. In this way, the SDG Dashboard highlights major implementation challenges in a goal even if the country performs well on several indicators. For example, a country that scores well on nutrition and food security indicators may be rated 'red' on SDG 2 if it experiences high rates of obesity or low nitrogen use efficiency.

\section{Data availability statement}

All data used for this study and results are available for download in Figshare at https://figshare.com/s/2a93a3dd3371157af033.

\section{Acknowledgements}

During several rounds of consultations hundreds of members of the SDSN, scientists; representatives of national statistical offices; United Nations, World Bank, and OECD; companies represented in the World Business Council for Sustainable Development; and civil society organizations have provided comments and advice on the SDG Index and Dashboards. Two anonymous reviewers provided valuable comments. Financial support was provided by the Bertelsmann Stiftung and the Sustainable Development Solutions Network.

\section{Author contributions}

All authors contributed extensively to the design of the SDG Index and Dashboards, and the methodology in the chapter. J.D.S. supervised the work. G.S-T. led the writing and revisions of the manuscript, with input from all other authors. K.T. and D.D-D. identified indicators, collected, checked and prepared data, wrote and ran code, and performed robustness tests, with input from all other authors at all stages. The initial concept for the SDG Index was developed by C.K. 


\section{Annex 2. Supplementary material}

This annex is based on Sachs et al. (2016b), drawing on OECD and JRC (2016).

\section{A2.1. Data selection}

\section{A2.1.1. Criteria for selecting indicators}

We identified technically-sound quantitative indicators for each SDG that met five statistical criteria for data selection (OECD and JRC, 2016; Booysen, 2002) to determine suitable metrics for inclusion in the SDG Index and Dashboards:

1. Global relevance and applicability to a broad range of country settings: The indicators were relevant for monitoring achievement of the SDGs and applicable to all or nearly all countries. They had to be internationally comparable and allow for direct comparison of performance across countries. They should allow for the definition of quantitative performance thresholds that signify SDG achievement.

2. Statistical adequacy: Data were collected and processed in a statistically reliable way without large or frequent revisions.

3. Timeliness: Data series had to be published on a reasonably prompt schedule and be available for most recent years.

4. Data quality: Data series had to be internationally comparable, represent the best available measure for a specific issue, and derive from official national or international sources (e.g. national statistical offices or United Nations organizations) or other reputable international sources.

5. Coverage: Data had to be available for at least $80 \%$ of the $149 \mathrm{UN}$ member states with a national population greater than 1 million.

\section{A2.1.2. Indicator selection}

The SDG Index was built on a set of indicators for each of the 17 SDGs using the most recent published data. All of the more than 230 official SDG indicators (IAEG-SDGs, 2016b) proposed by the Inter-Agency and Expert Group on SDG Indicators (IAEGSDGs) that met the five criteria were included in the SDG Index. Some official SDG indicators have adequate data coverage, but could not be included in the SDG Index and Dashboards as they did not permit a ranking of countries or the definition of a quantitative thresholds signifying achievement of the goals applicable to all countries (criterion 1 above). For example, different countries specialize in different sectors of the economy, so there is no 'right' threshold of manufacturing as a share of GDP that all countries should aim for. While individual countries may find the share of manufacturing value added highly useful for developing long-term strategies for (re-)industrialization, it is not possible to define a common global threshold for the SDGs. Other official SDG indicators are similarly useful at the country level, but cannot serve as a yardstick for comparing countries' performance internationally.

Where official SDG indicators did not meet the criteria for data selection or where indicator gaps remained, we considered official and other metrics published in the peerreviewed literature, as well as major databases and reports on development and environmental indicators (Kroll, 2015; SDSN, 2015a; UNDP, 2015; OECD, 2016b; World 
Bank, 2016b). We also consulted with a broad range of experts and conducted a public consultation on an earlier draft of the analysis that generated 56 submissions (Sachs et al., 2016a).

We were able to include 63 global indicators from a broad range of data sources (Table 8). The global SDG Index comprises 149 of the 193 UN member states (Table 12). OECD countries have more accurate and better data available across a wide range of indicators, so we included 14 additional variables for these countries in the global SDG Index to create an augmented SDG Index for OECD countries (Table 8).

\section{A2.1.3. Missing data}

The purpose of the SDG Index and Dashboards is to guide countries' discussions of their SDG priorities today based on available and robust data. For this reason, and since many SDG priorities lack widely accepted statistical models for imputing countrylevel data, we generally did not impute or model any missing data. We made exceptions for the following four variables that would otherwise not have been included because of missing data:

- Poverty headcount ratio at $\$ 1.90$ a day (2011 PPP) (\% of population): The World Bank (Ferreira et al., 2015) assumes zero extreme income poverty in high-income countries when constructing its global estimate of the number of people living below $\$ 1.90$ a day. We therefore assumed a value of $0 \%$ for all high-income countries where data were missing.

- Prevalence of undernourishment (\% of population): FAO et al. (2015a) report 14.7 million undernourished people in developed regions, which corresponds to an average prevalence of $1.2 \%$ in the developed regions. We therefore assumed a $1.2 \%$ prevalence rate for each high-income country (World Bank, 2016a) with missing data.

- Research and development expenditure (\% of GDP): We assumed zero R\&D expenditure for low-income countries that did not report any data for this variable.

- Percentage of children 5-14 years involved in child labor: The best performing upper-middle-income countries have a child labor rate of $1 \%$ (UNICEF, 2015). We assumed $0 \%$ child labor for high-income countries for which no data were reported.

For several indicators included in the SDG Index data are missing for some countries (see online data). Raw data included in the construction of the SDG Index and Dashboards are available for download with this thesis. Since the SDG Index compares countries it is important to avoid excessive bias through missing variables. The SDG Index therefore only includes countries that have data for at least $80 \%$ of the variables included in the global SDG Index or the Augmented SDG Index for OECD countries. All OECD countries had sufficient data for inclusion, and 149 countries met this test globally, including several countries with a national population less than 1 million. Table 12 lists the countries that were not included in this version of the SDG Index.

Among the countries excluded from the SDG Index and Dashboards were 31 small countries with populations of less than 1 million people. Countries with insufficient data availability fell into the following income categories, as defined by the World Bank (World Bank, 2016a): 12 high-income countries, 14 upper-middle- income countries, 8 
lower-middle-income countries, and 6 low-income countries. Many of these countries face major challenges in achieving the SDGs, so investing in their capacity to generate high-quality data is a priority for establishing better SDG baselines to eventually inform policy priorities and resource

\section{A2.2. Method for constructing the SDG Index}

The procedure for calculating the SDG Index comprised four steps: (i) perform statistical tests for normality and truncate extreme values from the distribution of each indicator; (ii) rescale the data to ensure comparability across indicators; (iii) aggregate the indicators within and across SDGs; and (iv) conduct sensitivity and other statistical tests on the SDG Index. We describe each of these steps below.

\section{A2.2.1. Statistical tests and truncation of extreme values}

Using a broad array of indicators presented in Table 9, we conducted several statistical tests to determine whether the variables considered in the SDG Index were normally distributed. These tests include skewness and kurtosis tests for normality as well as Shapiro-Wilk (Shapiro and Wilk, 1965) and Shapiro-Francia tests. For most indicators, we could reject the normality hypothesis at the $5 \%$ significance level. Often the deviation from normality was substantial, rendering some common statistical techniques invalid. Results for Shapiro-Wilk tests are presented in Table 10.

Since the underlying data were not normally distributed, we did not use Z-scores to construct the composite indices even though this is the most commonly used method (OECD, 2016b). A related reason for not using Z-scores was that the objective of the SDGs is for all countries to achieve common goal thresholds, including universal coverage of basic infrastructure and social services. If the SDGs are achieved then the data distribution will therefore diverge from the normal distribution underlying Z-scores. Moreover, while Z-scores track relative performance of countries by specifying the relative location of each measurement within a certain interval, a key purpose of the SDG Index is to show how far a country is from reaching quantitative thresholds associated with achieving the SDGs.

\section{A2.2.2. Rescaling and addressing extreme values}

To make the data comparable across indicators, each variable was rescaled from 0 to 100 with 0 denoting worst performance and 100 describing the optimum. Rescaling is usually very sensitive to the choice of limits and extreme values (outliers) at both tails of the distribution. The latter may become unintended thresholds and introduce spurious variability in the data. Consequently, the choice of upper and lower bounds can affect the relative ranking of countries in the index. This applies in particular to the lower bounds that affect the value and the units of the variable, which may in turn affect rankings, while the upper bound only affects the units (Booysen, 2002; OECD and JRC, 2016).

Where possible we used absolute goal thresholds to denote the upper bound for each distribution. These were derived from technically feasible maxima or goal thresholds for the indicator in question. For example, the upper bound for access to basic infrastructure was set at $100 \%$, and gender variables were bounded at perfect equality between men and women. For some variables, no absolute upper bounds could be identified in this way as it might be technically impossible to achieve certain absolute limits (e.g. zero deaths from road accidents, or zero Gini index). In such cases we 
considered the average of the five best performers among countries as the upper bound. Each indicator distribution was then truncated at the upper bound.

In some cases, the upper bound exceeded the thresholds to be met by 2030 to achieve the SDGs. For example, the SDGs call for reducing child mortality to no more than 25 per 1000 live births, but many countries have already exceeded this threshold (i.e. have mortality rates under 25 per 1000). By defining the upper bound as the 'best' outcome (e.g. 0 mortality per 1000) - not the SDG achievement threshold - the SDG Index rewards improvements across the full distribution. This is particularly important for countries that have already achieved some SDG thresholds, but still lag other countries on this metric. See Table 11 for a full description of the bounds used for each variable.

To remove the effect of extreme values, which can skew the results of a composite index, the OECD and JRC (2016) recommend truncating the data by removing the bottom 2.5 percentiles from the distribution. We applied this approach to the lower bound and truncated data at this level.

After establishing the upper and lower bounds, variables were transformed linearly to a scale between 0 and 100 using the following rescaling formula for the range [0;100]:

$$
\mathrm{x}^{\prime}=\frac{\mathrm{x}-\min (\mathrm{x})}{\max (\mathrm{x})-\min (\mathrm{x})}
$$

where $\mathrm{x}$ is the raw data value; $\max / \mathrm{min}$ denote the bounds for best and worst performance, respectively; and $x^{\prime}$ is the normalized value after rescaling.

The rescaling formula (Equation 2) ensured that all rescaled variables were expressed as ascending variables (i.e. higher values denoted better performance). In this way, the rescaled data became easy to interpret and compare across all indicators: a country that scores 50 on a variable is half-way towards achieving the optimum value; a country with a score of 75 has covered three quarters of the distance from worst to best.

\section{A2.2.3. Weighting and aggregation}

To arrive at a composite SDG Index, the constituent components needed to be weighted and aggregated. Different weightings of individual SDGs can have important implications on countries' performance and relative rankings in an SDG Index (Booysen, 2002). This issue is further compounded by the fact that the SDGs combine policy means (e.g. official development assistance) and policy ends (e.g. healthy life expectancy).

The results of several rounds of expert consultations on earlier drafts of the SDG Index made clear that there was no consensus across different epistemic communities on assigning higher weights to some SDGs over others. This confirms experiences with other composite indices that there is no universally agreed answer to this 'weighting problem' (Booysen, 2002). Some composite indices, such as the OECD Better Life Index (OECD, 2015a), therefore allow users to select the weights they attach to the components of an index. Such a flexible weighting methodology can be appropriate for measures of well-being because each user has an immediate and subjective experience of what a 'better life' means for her or him. In contrast, the SDGs describe a broad spectrum of scientific and policy challenges that few individuals or institutions master in their full breadth. Moreover, flexible weightings might encourage countries to "cherry- 
pick' the SDGs that are easier to achieve and leave aside equally important ones that require deeper transformations. For these reasons, we considered subjective and flexible weightings less suitable for the SDG Index.

As a normative assumption, we therefore opted for fixed weights and decided to give equal weight to every SDG to reflect policymakers' commitment to treat all SDGs equally and as an "integrated and indivisible" set of goals (United Nations, 2015a). This implies that to improve their SDG Index score countries need to place attention on all goals with a focus on goals where they are furthest from achieving the SDGs and where incremental progress might therefore be expected to be fastest.

Consequently, the aggregation for the SDG Index proceeded in two steps. First, the rescaled variables were combined for each SDG before being aggregated across goals. This approach also allows for the later addition of new variables for any SDG without affecting the relative weight of each SDG in the overall score.

Just like the weighting, the method for aggregating different variables into a single index can have profound implications on the overall ranking (OECD and JRC, 2016; Rickels et al., 2014). To allow for maximum flexibility in aggregating data, one can use the standard constant-elasticity-of-substitution (CES) function (Arrow et al., 1961; Blackorby and Donaldson, 1982) (Equation 3) to generate the SDG Index score $\mathrm{I}_{\mathrm{ij}}$ for SDG j and country i.

$$
I_{i j}\left(N_{i j}, I_{i j k}, \rho\right)=\left[\sum_{k=1}^{N_{i j}} \frac{1}{N_{i j}} I_{i j k}^{-\rho}\right]^{-\frac{1}{\rho}}
$$

Where $\mathrm{I}_{\mathrm{ijk}}$ is the score of indicator $\mathrm{k}$ under SDG $\mathrm{j}$ for country $\mathrm{i} ; \mathrm{N}_{\mathrm{ij}}$ denotes the number of indicators for SDG $\mathrm{j}$; and $\rho$ describes the substitutability across components of the indicator with a permissible range of $-1 \leq \rho \leq \infty$ (Arrow et al., 1961). An equivalent CES equation is used to aggregate the SDG Index scores $I_{i j}$ for country $i$ into the overall country score $\mathrm{I}_{\mathrm{j}}$.

The elasticity of substitution $\sigma$ across components of the SDG Index is defined as:

$$
\sigma=\frac{1}{1+\rho}
$$

With $0 \leq \sigma \leq \infty$ and

$$
\rho=\frac{1-\sigma}{\sigma}
$$

Three special cases of this CES function are frequently considered. First, if the components of the aggregate index are perfect substitutes $(\sigma=\infty, \rho=-1)$ then regress on one indicator (e.g. Gini index) can be offset by progress on another indicator (e.g. child mortality rate). This case is often referred to as 'weak sustainability'. The CES function with equal weights across components then assumes the form of the arithmetic mean:

$$
I_{i j}\left(N_{i j}, I_{i j k}\right)=\sum_{k=1}^{N_{i j}} \frac{1}{N_{i j}} I_{i j k}
$$


Second, strong sustainability occurs when the components of the SDG Index are not substitutable $(\sigma=0, \rho=\infty)$. In this case the CES function turns into a Leontief production function with orthogonal isoquants where the score $I_{i j}$ of a country $i$ and SDG $j$ is determined by the country's lowest score $\mathrm{I}_{\mathrm{ijk}}$ across all SDG indicators $\mathrm{k}$ :

$$
\mathrm{I}_{\mathrm{ij}}\left(\mathrm{I}_{\mathrm{ijk}}\right)=\operatorname{Min}\left\{\mathrm{I}_{\mathrm{ijk}}\right\}
$$

Finally, an intermediate case of linear substitutability is given by the Cobb-Douglas production function with $\sigma=1$ and $\rho=1$. In this case the SDG Index $\mathrm{I}_{\mathrm{ij}}$ becomes the geometric mean of the indices $\mathrm{I}_{\mathrm{ijk}}$ :

$$
I_{i j}\left(N_{i j}, I_{i j k}\right)=\prod_{k=1}^{N_{i j}} \sqrt[N_{i j}]{I_{i j k}}
$$

The geometric mean is often used to aggregate heterogeneous variables with limited substitutability and in cases where the focus of the analysis is on percentage changes instead of absolute changes. A prominent example is the Human Development Index (HDI), which changed its method of aggregation across three dimensions from arithmetic to geometric mean in 2010 (UNDP, 2015).

To aggregate indicator scores within each SDG we used the arithmetic mean ('weak sustainability') because each SDG describes a set of broadly complementary policy priorities. This implies that countries are indifferent to adding a unit of progress on any of the indicators comprised under an SDG. In line with our method for weighting across goals, each indicator was weighted equally. As a result, the relative weight of each indicator in a goal was inversely proportional to the number of indicators considered under that goal.

We considered all three options (arithmetic mean, geometric average, and Leontief function) for aggregating SDG scores $\mathrm{I}_{\mathrm{ij}}$ across SDGs $\mathrm{j}$. Since the SDGs are an integrated and indivisible agenda requiring progress towards all goals, perfect substitutability across goals, as required for using the arithmetic mean, cannot be assumed outright. The geometric average has the advantage of reflecting an assumed 'penalty' on very low scores, unlike the arithmetic mean. Meanwhile, the Leontief minimum function focuses on the single SDG where a country performs worst, which is a poor indication of how the country performs across the 17 goals. We therefore considered both the arithmetic and geometric averages as two plausible approaches. Both yielded similar results with a correlation coefficient of 0.977 and very similar rankings (Table 14).

Compared with the geometric mean the arithmetic average has the advantage of simplicity of interpretation: an index score between 0 and 100 reflects the average initial placement of the country between worst and best on the average of the 17 goals. Based on the similarity of results confirmed by additional sensitivity tests (see below) and the greater ease of interpretation of the arithmetic mean we opted for the latter to aggregate goal indices $\mathrm{I}_{\mathrm{ij}}$ across SDGs $\mathrm{j}$.

A country's overall SDG Index score was therefore estimated by combining Equation 6 for aggregation within and across SDGs to yield Equation 9:

$$
I_{i}\left(N_{i}, N_{i j}, I_{i j k}\right)=\sum_{j=1}^{N_{i}} \frac{1}{N_{i}} \sum_{k=1}^{N_{i j}} \frac{1}{N_{i j}} I_{i j k}
$$


Where $I_{i}$ is the index score for country $i, N_{i}$ the number of SDGs for which the country has data, $\mathrm{N}_{\mathrm{ij}}$ the number of indicators for SDG $\mathrm{j}$ for which country $\mathrm{i}$ has data, and $\mathrm{I}_{\mathrm{ijk}}$ denotes the score of indicator $k$ under SDG $\mathrm{j}$ for country $\mathrm{i}$. The final index scores are reported in Table 7 and Table 15.

\section{A2.2.4. Sensitivity and other statistical tests on the SDG Index}

As a further robustness test, we calculated the median rank between the arithmetic and geometric ranks as shown in Table 14. The volatility between ranks is limited. Only several countries have more than 10 positions difference between the arithmetic and the median rank. These differences were due to the property of geometric mean, unlike the arithmetic mean, to penalize very low scores on specific goals. As a result, countries at the bottom of the SDG Index distribution obtain extremely low overall scores with the geometric mean. This finding further corroborated our decision to opt for the simpler and easier-to-interpret arithmetic mean.

To test the robustness of the upper and lower bounds used for the normalization of variables (Equation 2), we considered alternative approaches to setting 'worst' (=0) performance. As an alternative to truncating the datasets at the 2.5 percentile to establish the lower bound, we considered truncation at the 5 th percentile, as well as using the average of the bottom 5 performers as the lower bound. Upper bounds were left unchanged since they reflect the goal benchmarks to be achieved by 2030 . The resulting rankings (Table 13) show that only a limited number of country rankings are affected by the method for setting upper and lower bounds. We conclude that our findings are robust to the specification of the lower bounds.

\section{A2.3. Method for constructing the SDG Dashboards}

The SDG Dashboards use the same data as the SDG Index after truncation and rescaling. We introduced additional quantitative thresholds for each indicator to group countries in a 'traffic-light' table. Aggregating across all indicators for a goal yielded an overall score for each SDG and each country.

\section{A2.3.1. Thresholds}

To assess a country's progress on a particular indicator, we considered three bands (i) the green band is bounded by the maximum that can be achieved for each variable (i.e. the upper bound) and the threshold for achieving the SDG; (ii) an intermediate yellow band is bounded by SDG achievement at the top and a threshold denoting significant challenges in achieving the SDGs; and (iii) the red band is bounded at the bottom by the lower bound denoting worst performance in the sample truncated at 2.5 percentile. Upper and lower bounds are the same as for the SDG Index. Additional thresholds were established in consultation with experts, and the country assessments were subject to a public consultation(Sachs et al., 2016a) and direct consultations with members of the Sustainable Development Solutions Network. All thresholds were specified in absolute terms and applied to all countries (Table 11).

\section{A2.3.2. Weighting and aggregation}

The purpose of the SDG Dashboards is to highlight those SDGs that require particular attention in each country and therefore should be prioritized for early action. For the design of the SDG Dashboards, the same issues related to weighting and aggregation of indicators apply, as discussed above for the SDG Index. 
Averaging across all indicators for an SDG might hide areas of policy concern if a country performs well on most indicators but faces serious shortfalls on one or two metrics within the same SDG. This applies particularly to high-income and upper-middle-income countries that have achieved significant progress on many SDG dimensions but may face serious shortfalls on individual variables.

The SDG Dashboards therefore used the Leontief Minimum function (Equation 7) to aggregate indicator scores for each SDG. The score for each goal was determined by the variable on which the country performs worst. This approach highlights the gaps in SDG achievement rather than average progress as under the SDG Index.

SDG Dashboards highlight major challenges across most SDGs for poorer countries, particularly in sub-Saharan Africa. We therefore considered using different aggregation methodologies for OECD and non-OECD countries, such as the minimum function and the arithmetic mean. However, such different approaches yielded stark differences in results between countries that were at the intersection of both groups. In particular, non-OECD high-income or upper-middle-income countries ended up with significantly fewer 'red' SDGs than their peers inside the OECD. To avoid such arbitrary distinctions, we resolved to use the same methodology for all countries.

As with the SDG Index, every UN member country with data for at least $80 \%$ of the variables was included in the SDG Dashboards (Table 12). Since ocean data for SDG 14 were only available for countries with a seashore, we applied the $80 \%$ threshold to the 116 non-landlocked countries with a population greater than 1 million.

OECD countries have access to more data and possess the resources to make rapid progress towards achieving each SDGs, so we included additional variables in separate Dashboards for OECD countries. Since the OECD produces better and more easily comparable data on unemployment than is available internationally, the corresponding indicator for the Dashboards for OECD countries replaced the variable used in the global SDG Dashboards. Similarly, the indicator on municipal solid waste was replaced by an indicator factoring in recycling rates (Table 8 ).

\section{A2.4. Methodological and data limitations}

There are several limitations of this first global effort to establish internationally comparable country baselines for the SDGs.

An equal weighting of all SDGs, as proposed in the SDG Index, will lead higher-income countries to perform better on average: These countries tend to perform better on most economic and social SDG priorities. They also perform better on some local environmental priorities, including access to wastewater treatment, deforestation rates, and rates of biodiversity loss. Rich countries perform worse on greenhouse gas emissions and some metrics for sustainable consumption and production, but these represent a small share of SDG indicators considered in this SDG Index. Consequently, the results of the SDG Index may be biased towards richer countries.

A few SDGs and their targets focus on cross-country effects or global public goods. For example, SDG 10 calls for reducing inequality within and between countries. The SDG Index and Dashboards consider each country separately and therefore cannot track progress towards reducing inequality between countries or promoting global public goods. Such SDG priorities will require other analytical tools not included here. 
A closely related point is that action inside some countries can have a significant impact on other countries' ability to achieve the SDGs. Some of these effects are captured in the SDG Index and Dashboards (e.g. international development finance, or per capita greenhouse gas emissions), but many are not. Such cross-border effects might include demand for environmental resources in rich countries that accelerate environmental degradation in developing countries or the effect of rules and standards for international trade.

Many proposed official SDG indicators lack data for the majority of countries and could therefore not be included in this SDG Index and Dashboards. Other countries lack data for some SDGs, which introduces a bias in the computation of the SDG Index and Dashboards.

Owing to limited data availability and lack of metrics for key SDG priorities, this first SDG Index presents an incomplete picture of SDG baselines. As data availability improves and new estimation techniques become available, subsequent editions of the SDG Index and Dashboard may include additional variables. Not all data for SDG Indicators are updated annually and at the same time for all countries. Household and other surveys are conducted infrequently and at different times across countries. Moreover, the data might become available with lags of several years, as is the case with estimates of headcount poverty rates. As a result, updated editions of the SDG Index may not be fully comparable with earlier versions.

We used the most recent available data for each indicator and did not consider historical data since the availability of such time series data was too limited for most variables with metrics for economic development and health being notable exceptions. Estimating year-on-year changes would require modelling of all variables, as done for health by the Global Burden of Disease Collaboration (GBD, 2016). Yet, data of comparable quality and models of sufficient robustness were not available for a sufficient number of SDG priority areas to estimate trend data for the overall SDG Index. As a result, we could not infer how fast countries had been progressing towards achieving the SDGs.

We opted against including momentum data available for a subset of SDG metrics in the first version of the SDG Index since doing so would have skewed the analysis towards increasing the weight of variables for which such trend data were available. Modelling across time and countries for all SDG priorities would require major efforts that could not be accomplished for this edition of the SDG Index and Dashboards. Moreover, it would risk diverting attention away from increasing investments to fill current data gaps.

In addition, the SDG Dashboards do not yet capture important regional challenges that are less relevant at the global level, such as neglected tropical diseases, malaria, or inequality in education outcomes. Similarly, most Small-Island-Developing States lack adequate data for inclusion as described in Table 12 in the global SDG Index and Dashboards, so tools could be developed that better address the specific needs of these countries. 


\section{A2.5. Annex 2 tables}

Table 6 | The Sustainable Development Goals

\begin{tabular}{|c|c|}
\hline 1 than & Goal 1. End poverty in all its forms everywhere \\
\hline 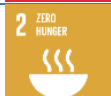 & $\begin{array}{l}\text { Goal } 2 \text {. End hunger, achieve food security and improved nutrition and pro- } \\
\text { mote sustainable agriculture }\end{array}$ \\
\hline 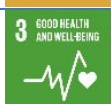 & Goal 3. Ensure healthy lives and promote well-being for all at all ages \\
\hline & $\begin{array}{l}\text { Goal } 4 \text {. Ensure inclusive and equitable quality education and promote lifelong } \\
\text { learning opportunities for all }\end{array}$ \\
\hline 5 sacun & Goal 5. Achieve gender equality and empower all women and girls \\
\hline 6 & $\begin{array}{l}\text { Goal } 6 \text {. Ensure availability and sustainable management of water and sani- } \\
\text { tation for all }\end{array}$ \\
\hline & $\begin{array}{l}\text { Goal } 7 \text {. Ensure access to affordable, reliable, sustainable and modern energy } \\
\text { for all }\end{array}$ \\
\hline & $\begin{array}{l}\text { Goal 8. Promote sustained, inclusive and sustainable economic growth, full } \\
\text { and productive employment and decent work for all }\end{array}$ \\
\hline & $\begin{array}{l}\text { Goal 9. Build resilient infrastructure, promote inclusive and sustainable indus- } \\
\text { trialization and foster innovation }\end{array}$ \\
\hline E & Goal 10. Reduce inequality within and among countries \\
\hline & $\begin{array}{l}\text { Goal 11. Make cities and human settlements inclusive, safe, resilient and } \\
\text { sustainable }\end{array}$ \\
\hline Q & Goal 12. Ensure sustainable consumption and production patterns \\
\hline 13 & Goal 13. Take urgent action to combat climate change and its impacts* \\
\hline & $\begin{array}{l}\text { Goal 14. Conserve and sustainably use the oceans, seas and marine re- } \\
\text { sources for sustainable development }\end{array}$ \\
\hline$S_{15}^{15}$ & $\begin{array}{l}\text { Goal } 15 \text {. Protect, restore and promote sustainable use of terrestrial ecosys- } \\
\text { tems, sustainably manage forests, combat desertification, and halt and re- } \\
\text { verse land degradation and halt biodiversity loss }\end{array}$ \\
\hline 16 & $\begin{array}{l}\text { Goal 16. Promote peaceful and inclusive societies for sustainable develop- } \\
\text { ment, provide access to justice for all and build effective, accountable and } \\
\text { inclusive institutions at all levels }\end{array}$ \\
\hline 8 & $\begin{array}{l}\text { Goal 17. Strengthen the means of implementation and revitalize the Global } \\
\text { Partnership for Sustainable Development }\end{array}$ \\
\hline
\end{tabular}


Table 7 | SDG Index country rankings and scores

SDG Index ranking and scores (0 to 100) for 149 countries included in the 2016 SDG Index.

\begin{tabular}{|c|c|c|}
\hline Rank & Country & Score \\
\hline 1 & Sweden & 84.5 \\
\hline 2 & Denmark & 83.9 \\
\hline 3 & Norway & 82.3 \\
\hline 4 & Finland & 81.0 \\
\hline 5 & Switzerland & 80.9 \\
\hline 6 & Germany & 80.5 \\
\hline 7 & Austria & 79.1 \\
\hline 8 & Netherlands & 78.9 \\
\hline 9 & Iceland & 78.4 \\
\hline 10 & United Kingdom & 78.1 \\
\hline 11 & France & 77.9 \\
\hline 12 & Belgium & 77.4 \\
\hline 13 & Canada & 76.8 \\
\hline 14 & Ireland & 76.7 \\
\hline 15 & Czech Republic & 76.7 \\
\hline 16 & Luxembourg & 76.7 \\
\hline 17 & Slovenia & 76.6 \\
\hline 18 & Japan & 75.0 \\
\hline 19 & Singapore & 74.6 \\
\hline 20 & Australia & 74.5 \\
\hline 21 & Estonia & 74.5 \\
\hline 22 & New Zealand & 74.0 \\
\hline 23 & Belarus & 73.5 \\
\hline 24 & Hungary & 73.4 \\
\hline 25 & United States & 72.7 \\
\hline 26 & Slovak Republic & 72.7 \\
\hline 27 & South Korea & 72.7 \\
\hline 28 & Latvia & 72.5 \\
\hline 29 & Israel & 72.3 \\
\hline 30 & Spain & 72.2 \\
\hline 31 & Lithuania & 72.1 \\
\hline 32 & Malta & 72.0 \\
\hline 33 & Bulgaria & 71.8 \\
\hline 34 & Portugal & 71.5 \\
\hline 35 & Italy & 70.9 \\
\hline 36 & Croatia & 70.7 \\
\hline 37 & Greece & 69.9 \\
\hline 38 & Poland & 69.8 \\
\hline 39 & Serbia & 68.3 \\
\hline 40 & Uruguay & 68.0 \\
\hline 41 & Romania & 67.5 \\
\hline 42 & Chile & 67.2 \\
\hline 43 & Argentina & 66.8 \\
\hline 44 & Moldova & 66.6 \\
\hline 45 & Cyprus & 66.5 \\
\hline 46 & Ukraine & 66.4 \\
\hline 47 & Russian Federation & 66.4 \\
\hline 48 & Turkey & 66.1 \\
\hline 49 & Qatar & 65.8 \\
\hline 50 & Armenia & 65.4 \\
\hline 51 & Tunisia & 65.1 \\
\hline 52 & Brazil & 64.4 \\
\hline 53 & Costa Rica & 64.2 \\
\hline 54 & Kazakhstan & 63.9 \\
\hline
\end{tabular}

\begin{tabular}{|c|c|c|}
\hline Rank & Country & Score \\
\hline 55 & United Arab Emirates & 63.6 \\
\hline 56 & Mexico & 63.4 \\
\hline 57 & Georgia & 63.3 \\
\hline 58 & Macedonia, FYR & 62.8 \\
\hline 59 & Jordan & 62.7 \\
\hline 60 & Montenegro & 62.5 \\
\hline 61 & Thailand & 62.2 \\
\hline 62 & Venezuela, RB & 61.8 \\
\hline 63 & Malaysia & 61.7 \\
\hline 64 & Morocco & 61.6 \\
\hline 65 & Azerbaijan & 61.3 \\
\hline 66 & Egypt, Arab Rep. & 60.9 \\
\hline 67 & Kyrgyz Republic & 60.9 \\
\hline 68 & Albania & 60.8 \\
\hline 69 & Mauritius & 60.7 \\
\hline 70 & Panama & 60.7 \\
\hline 71 & Ecuador & 60.7 \\
\hline 72 & Tajikistan & 60.2 \\
\hline 73 & $\begin{array}{l}\text { Bosnia and } \\
\text { Herzegovina }\end{array}$ & 59.9 \\
\hline 74 & Oman & 59.9 \\
\hline 75 & Paraguay & 59.3 \\
\hline 76 & China & 59.1 \\
\hline 77 & Jamaica & 59.1 \\
\hline 78 & Trinidad and Tobago & 59.1 \\
\hline 79 & Iran, Islamic Rep. & 58.5 \\
\hline 80 & Botswana & 58.4 \\
\hline 81 & Peru & 58.4 \\
\hline 82 & Bhutan & 58.2 \\
\hline 83 & Algeria & 58.1 \\
\hline 84 & Mongolia & 58.1 \\
\hline 85 & Saudi Arabia & 58.0 \\
\hline 86 & Lebanon & 58.0 \\
\hline 87 & Suriname & 58.0 \\
\hline 88 & Vietnam & 57.6 \\
\hline 89 & Bolivia & 57.5 \\
\hline 90 & Nicaragua & 57.4 \\
\hline 91 & Colombia & 57.2 \\
\hline 92 & Dominican Republic & 57.1 \\
\hline 93 & Gabon & 56.2 \\
\hline 94 & El Salvador & 55.6 \\
\hline 95 & Philippines & 55.5 \\
\hline 96 & Cabo Verde & 55.5 \\
\hline 97 & Sri Lanka & 54.8 \\
\hline 98 & Indonesia & 54.4 \\
\hline 99 & South Africa & 53.8 \\
\hline 100 & Kuwait & 52.5 \\
\hline 101 & Guyana & 52.4 \\
\hline 102 & Honduras & 51.8 \\
\hline 103 & Nepal & 51.5 \\
\hline 104 & Ghana & 51.4 \\
\hline 105 & Iraq & 50.9 \\
\hline 106 & Guatemala & 50.0 \\
\hline 107 & Lao PDR & 49.9 \\
\hline
\end{tabular}


On metrics and financing for the Sustainable Development Goals

\begin{tabular}{|r|l|r|}
\hline Rank & Country & Score \\
\hline 108 & Namibia & 49.9 \\
\hline 109 & Zimbabwe & 48.6 \\
\hline 110 & India & 48.4 \\
\hline 111 & Congo, Rep. & 47.2 \\
\hline 112 & Cameroon & 46.3 \\
\hline 113 & Lesotho & 45.9 \\
\hline 114 & Senegal & 45.8 \\
\hline 115 & Pakistan & 45.7 \\
\hline 116 & Swaziland & 45.1 \\
\hline 117 & Myanmar & 44.5 \\
\hline 118 & Bangladesh & 44.4 \\
\hline 119 & Cambodia & 44.4 \\
\hline 120 & Kenya & 44.0 \\
\hline 121 & Angola & 44.0 \\
\hline 122 & Rwanda & 44.0 \\
\hline 123 & Uganda & 43.6 \\
\hline 124 & Cote d'Ivoire & 43.5 \\
\hline 125 & Ethiopia & 43.1 \\
\hline 126 & Tanzania & 43.0 \\
\hline 127 & Sudan & 42.2 \\
\hline 128 & Burundi & 42.0 \\
\hline 129 & Togo & 40.9 \\
\hline
\end{tabular}

\begin{tabular}{|r|l|r|}
\hline Rank & Country & Score \\
\hline 130 & Benin & 40.0 \\
\hline 131 & Malawi & 39.8 \\
\hline 132 & Mauritania & 39.6 \\
\hline 133 & Mozambique & 39.5 \\
\hline 134 & Zambia & 38.4 \\
\hline 135 & Mali & 38.2 \\
\hline 136 & Gambia, The & 37.8 \\
\hline 137 & Yemen, Rep. & 37.3 \\
\hline 138 & Sierra Leone & 36.9 \\
\hline 139 & Afghanistan & 36.5 \\
\hline 140 & Madagascar & 36.2 \\
\hline 141 & Nigeria & 36.1 \\
\hline 142 & Guinea & 35.9 \\
\hline 143 & Burkina Faso & 35.6 \\
\hline 144 & Haiti & 34.4 \\
\hline 145 & Chad & 31.8 \\
\hline 146 & Niger & 31.4 \\
\hline 147 & Congo, Dem. Rep. & 31.3 \\
\hline 148 & Liberia & 30.5 \\
\hline 149 & Central African & 26.1 \\
& Republic & \\
\hline
\end{tabular}


Table 8 | Indicators used in the SDG Index and Dashboards

Description of indicators used in the global SDG Index and Dashboards. Indicators used only in the Augmented SDG Index and Dashboards for OECD countries are marked (a) or (b), respectively, denoting an addition or the replacement of a corresponding indicator from the global indicator set. Indicators that are identical or similar to indicators in the official UNSTATS database are noted as • and o respectively.

\begin{tabular}{|c|c|c|c|c|c|}
\hline SDG & Indicator & $\begin{array}{l}\text { OECD-only } \\
\text { indicators }\end{array}$ & $\begin{array}{l}\text { Official } \\
\text { indicators }\end{array}$ & $\begin{array}{l}\text { Latest } \\
\text { available } \\
\text { year }\end{array}$ & Source \\
\hline \multirow[t]{2}{*}{1} & $\begin{array}{l}\text { Poverty headcount ratio at } \$ 1.90 \text { a day (2011 } \\
\text { PPP) ( } \% \text { of population) }\end{array}$ & & - & $2009-2013$ & World Bank (2016c) \\
\hline & $\begin{array}{l}\text { Poverty rate after taxes and transfers, poverty } \\
\text { line } 50 \% \text { (\% of population) }\end{array}$ & (a) & - & $2011-2014$ & OECD (2016b) \\
\hline \multirow[t]{6}{*}{2} & $\begin{array}{l}\text { Prevalence of undernourishment (\% of popula- } \\
\text { tion) }\end{array}$ & & $\bullet$ & 2013 & FAO (2015) \\
\hline & Cereal yield (t/ha) & & - & 2013 & FAO (2017a) \\
\hline & $\begin{array}{l}\text { Prevalence of stunting (low height-for-age) in } \\
\text { children under } 5 \text { years of age }(\%)\end{array}$ & & $\bullet$ & $2000-2015$ & UNICEF et al. (2017a) \\
\hline & $\begin{array}{l}\text { Prevalence of wasting in children under } 5 \text { years } \\
\text { of age }(\%)\end{array}$ & & $\bullet$ & $2000-2015$ & UNICEF et al. (2017a) \\
\hline & Sustainable Nitrogen Management Index (0-1) & & - & $2006 / 2011$ & $\begin{array}{l}\text { Zhang and Davidson (2016), } \\
\text { Zhang et al. (2015) }\end{array}$ \\
\hline & $\begin{array}{l}\text { Prevalence of obesity, BMI } \geq 30 \text { ( } \% \text { of adult pop- } \\
\text { ulation) }\end{array}$ & (a) & - & 2014 & WHO (2017a) \\
\hline \multirow[t]{11}{*}{3} & Mortality rate, under-5 (per 1,000 live births) & & $\bullet$ & 2013 & UNICEF et al. (2017b) \\
\hline & Maternal mortality rate (per 100,000 live births) & & - & 2015 & WHO (2017b) \\
\hline & Neonatal mortality rate (per 1000 live births) & & • & 2015 & UNICEF et al. (2017c) \\
\hline & Physician density (per 1000 people) & & $\bullet$ & $2004-2013$ & WHO (2016a) \\
\hline & Incidence of tuberculosis (per 100,000 people) & & - & 2014 & WHO (2017c) \\
\hline & Traffic deaths rate (per 100,000 people) & & $\bullet$ & 2013 & WHO (2016b) \\
\hline & $\begin{array}{l}\text { Adolescent fertility rate (births per 1,000 women } \\
\text { ages 15-19) }\end{array}$ & & - & $2005-2015$ & UNDP (2017) \\
\hline & $\begin{array}{l}\text { Subjective wellbeing (average ladder score, } 0 \text { - } \\
\text { 10) }\end{array}$ & & - & 2014 & Helliwell et al. (2016b) \\
\hline & Healthy life expectancy at birth (years) & & - & 2015 & WHO (2017d) \\
\hline & $\begin{array}{l}\text { Percentage of surviving infants who received } 2 \\
\text { WHO-recommended vaccines (\%) }\end{array}$ & & - & 2014 & WHO and UNICEF (2016a) \\
\hline & Daily smokers ( $\%$ of population aged $15+)$ & (a) & • & $2006-2013$ & OECD (2016b) \\
\hline \multirow[t]{6}{*}{4} & Expected years of schooling (years) & & - & 2013 & UNDP (2016b) \\
\hline & Literacy rate of $15-24$ year olds, both sexes (\%) & & $\circ$ & $2001-2013$ & UNESCO (2017) \\
\hline & Net primary school enrolment rate (\%) & & ○ & $1997-2014$ & UNESCO (2017) \\
\hline & $\begin{array}{l}\text { Population aged } 25-64 \text { with tertiary education } \\
(\%)\end{array}$ & (a) & - & 2011 & OECD (2016b) \\
\hline & PISA score $(0-600)$ & (a) & - & 2012 & OECD (2016b) \\
\hline & $\begin{array}{l}\text { Population aged } 25-64 \text { with upper secondary } \\
\text { and post-secondary non-tertiary educational at- } \\
\text { tainment }(\%)\end{array}$ & (a) & - & $2011-2013$ & OECD (2016b) \\
\hline \multirow[t]{5}{*}{5} & $\begin{array}{l}\text { Proportion of seats held by women in national } \\
\text { parliaments (\%) }\end{array}$ & & $\bullet$ & $2012-2014$ & IPU (2016) \\
\hline & $\begin{array}{l}\text { Female years of schooling of population aged } 25 \\
\text { and above (\% male) }\end{array}$ & & - & 2014 & UN Women (2015) \\
\hline & Female labor force participation rate (\% male) & & - & $2010-2014$ & ILO (2016a) \\
\hline & $\begin{array}{l}\text { Estimated unmet demand for contraception (\% } \\
\text { of women married or in union, ages 15-49) }\end{array}$ & & $\bullet$ & 2015 & UNDESA (2017) \\
\hline & Gender wage gap (\% of male median wage) & (a) & - & 2012 & OECD (2016b) \\
\hline \multirow[t]{3}{*}{6} & $\begin{array}{l}\text { Access to improved water source (\% of popula- } \\
\text { tion) }\end{array}$ & & - & $2011-2015$ & WHO and UNICEF (2016b) \\
\hline & $\begin{array}{l}\text { Access to improved sanitation facilities (\% of } \\
\text { population) }\end{array}$ & & - & $2011-2015$ & WHO and UNICEF (2016b) \\
\hline & $\begin{array}{l}\text { Freshwater withdrawal (\% of total renewable wa- } \\
\text { ter resources) }\end{array}$ & & $\bullet$ & $1999-2012$ & FAO (2017b) \\
\hline \multirow[t]{4}{*}{7} & Access to electricity (\% of population) & & $\bullet$ & 2012 & SE4All (2017a) \\
\hline & Access to non-solid fuels ( $\%$ of population) & & o & 2010 & SE4All (2017b) \\
\hline & $\begin{array}{l}\mathrm{CO}_{2} \text { emissions from fuel combustion and elec- } \\
\text { tricity output }\left(\mathrm{MtCO}_{2} / \mathrm{TWh}\right)\end{array}$ & & - & 2013 & IEA (2016) \\
\hline & $\begin{array}{l}\text { Share of renewable energy in total final energy } \\
\text { consumption }(\%)\end{array}$ & (a) & $\bullet$ & 2010 & OECD et al. (2017) \\
\hline
\end{tabular}




\begin{tabular}{|c|c|c|c|c|c|}
\hline SDG & Indicator & $\begin{array}{l}\text { OECD-only } \\
\text { indicators }\end{array}$ & $\begin{array}{l}\text { Official } \\
\text { indicators }\end{array}$ & $\begin{array}{l}\text { Latest } \\
\text { available } \\
\text { year }\end{array}$ & Source \\
\hline \multirow[t]{6}{*}{8} & Unemployment rate ( $\%$ of total labor force) & (b) & - & 2015 & ILO (2016b) \\
\hline & $\begin{array}{l}\text { Automated teller machines (ATMs per 100,000 } \\
\text { adults) }\end{array}$ & & - & $2009-2014$ & IMF (2015a) \\
\hline & Adjusted growth rate (\%) & & $\circ$ & 2012 & OECD (2016b) \\
\hline & $\begin{array}{l}\text { Youth not in employment, education or training } \\
(\text { NEET) }(\%)\end{array}$ & (a) & $\bullet$ & 2013-2014 & OECD (2016b) \\
\hline & $\begin{array}{l}\text { Percentage of children 5-14 years old involved in } \\
\text { child labor }(\%)\end{array}$ & & $\bullet$ & $2000-2014$ & UNICEF (2015) \\
\hline & Employment-to-Population ratio (\%) & (a) & $\bullet$ & 2014 & OECD (2016b) \\
\hline \multirow[t]{7}{*}{9} & $\begin{array}{l}\text { Research and development expenditure (\% of } \\
\text { GDP) }\end{array}$ & & $\bullet$ & $2005-2012$ & UNESCO (2017) \\
\hline & $\begin{array}{l}\text { Research and development researchers (per } \\
1000 \text { employed) }\end{array}$ & (a) & ○ & $2010-2014$ & OECD (2016b) \\
\hline & $\begin{array}{l}\text { Logistics Performance Index: Quality of trade } \\
\text { and transport-related infrastructure }(1-5)\end{array}$ & & - & 2014 & World Bank. (2016d) \\
\hline & Quality of overall infrastructure (1-7) & & - & $2014 / 2015$ & WEF (2016) \\
\hline & $\begin{array}{l}\text { Mobile broadband subscriptions (per } 100 \text { inhabit- } \\
\text { ants) }\end{array}$ & & o & $2012-2015$ & ITU (2016) \\
\hline & $\begin{array}{l}\text { Proportion of the population using the internet } \\
(\%)\end{array}$ & & $\bullet$ & 2014 & ITU (2016) \\
\hline & $\begin{array}{l}\text { Patent applications filed under PCT in inventor's } \\
\text { country of residence (per million population) }\end{array}$ & (a) & - & 2012 & OECD (2016b) \\
\hline \multirow[t]{3}{*}{10} & Gini index $(0-100)$ & & - & $2003-2012$ & $\begin{array}{l}\text { World Bank (2017a), OECD } \\
(2016 \mathrm{~b})\end{array}$ \\
\hline & Palma ratio & (a) & - & $2009-2012$ & OECD (2016b) \\
\hline & PISA Social Justice Index (0-10) & (a) & - & 2012 & OECD (2017a) \\
\hline \multirow[t]{3}{*}{11} & $\begin{array}{l}\text { Annual mean concentration of particulate matter } \\
(\mathrm{PM} 2.5)\left(\mu \mathrm{g} / \mathrm{m}^{3}\right) \text { in urban areas }\end{array}$ & & $\bullet$ & 2013 & Forouzanfar et al. (2016) \\
\hline & Rooms per person & (a) & - & $2001-2013$ & OECD (2016b) \\
\hline & $\begin{array}{l}\text { Improved water source, piped (\% of urban popu- } \\
\text { lation with access) }\end{array}$ & & - & 2015 & WHO and UNICEF (2016b) \\
\hline \multirow[t]{3}{*}{12} & $\begin{array}{l}\text { Percentage of anthropogenic wastewater that re- } \\
\text { ceives treatment (\%) }\end{array}$ & & $\bullet$ & 2012 & OECD (2016b) \\
\hline & Municipal solid waste (kg/year/capita) & (b) & - & 2012 & World Bank (2012) \\
\hline & $\begin{array}{l}\text { Non-recycled municipal solid waste (kg/per- } \\
\text { son/year) }\end{array}$ & (a) & o & $2009-2013$ & OECD (2016b) \\
\hline \multirow[t]{2}{*}{13} & $\begin{array}{l}\text { Energy-related } \mathrm{CO}_{2} \text { emissions per capita } \\
\left(\mathrm{tCO}_{2} / \text { capita }\right)\end{array}$ & & - & 2011 & World Bank (2017b) \\
\hline & Climate Change Vulnerability Monitor $(0-1)$ & & - & 2014 & HCSS (2015) \\
\hline \multirow[t]{5}{*}{14} & Ocean Health Index Goal - Clean Waters $(0-100)$ & & o & 2015 & Ocean Health Index (2016) \\
\hline & Ocean Health Index Goal - Biodiversity (0-100) & & $\circ$ & 2015 & Ocean Health Index (2016) \\
\hline & Ocean Health Index Goal - Fisheries (0-100) & & 0 & 2015 & Ocean Health Index (2016) \\
\hline & $\begin{array}{l}\text { Marine sites of biodiversity importance that are } \\
\text { completely protected }(\%)\end{array}$ & & $\bullet$ & 2013 & $\begin{array}{l}\text { BirdLife International et al. } \\
(2017)\end{array}$ \\
\hline & $\begin{array}{l}\text { Percentage of fish stocks overexploited or col- } \\
\text { lapsed by EEZ }(\%)\end{array}$ & & $\bullet$ & 2010 & $\begin{array}{l}\text { Hsu et al. (2016), Sea Around } \\
\text { Us (2016) }\end{array}$ \\
\hline \multirow[t]{3}{*}{15} & Red List Index of species survival $(0-1)$ & & $\circ$ & 2016 & $\begin{array}{l}\text { IUCN and BirdLife Interna- } \\
\text { tional }(2017)\end{array}$ \\
\hline & Annual change in forest area (\%) & & o & 2012 & Hsu et al. (2016) \\
\hline & $\begin{array}{l}\text { Terrestrial sites of biodiversity importance that } \\
\text { are completely protected (\%) }\end{array}$ & & $\bullet$ & 2013 & $\begin{array}{l}\text { BirdLife International et al. } \\
(2017)\end{array}$ \\
\hline \multirow[t]{7}{*}{16} & Homicides (per 100,000 people) & & $\bullet$ & $2008-2012$ & UNODC (2016) \\
\hline & Prison population (per 100,000 people) & & - & $2002-2013$ & ICPR (2016) \\
\hline & $\begin{array}{l}\text { Proportion of population who feel safe walking } \\
\text { alone at night where they live (\%) }\end{array}$ & & $\bullet$ & $2006-2015$ & Gallup (2016) \\
\hline & Corruption Perception Index (0-100) & & - & 2014 & $\begin{array}{l}\text { Transparency International } \\
(2015)\end{array}$ \\
\hline & $\begin{array}{l}\text { Proportion of children under } 5 \text { whose births have } \\
\text { been registered with a civil authority, by age (\%) }\end{array}$ & & $\bullet$ & 2014 & UNICEF (2016) \\
\hline & Government efficiency (1-7) & & - & 2015/2016 & WEF (2016) \\
\hline & Property rights (1-7) & & - & $2014 / 2015$ & WEF (2016) \\
\hline \multirow[t]{3}{*}{17} & $\begin{array}{l}\text { For high-income and all OECD DAC countries: } \\
\text { International concessional public finance, includ- } \\
\text { ing ODA ( } \% \text { of GNI) }\end{array}$ & & $\bullet$ & 2013 & OECD (2015b) \\
\hline & For all other countries: Tax revenue ( $\%$ of GDP) & & $\bullet$ & 2013 & World Bank (2017b) \\
\hline & $\begin{array}{l}\text { Health, education and R\&D spending (\% of } \\
\text { GDP) }\end{array}$ & & - & $2005-2014$ & UNDP (2015) \\
\hline
\end{tabular}


Table 9 | Summary statistics for indicators included in SDG Index and Dashboards

Number of observations (N), statistical mean (Mean), standard deviation (SD), the minimum (Min) and maximum (Max) values across all countries with data availability. ${ }^{*}$ Indicator only included in augmented SDG Index for OECD countries.

\begin{tabular}{|c|c|c|c|c|c|c|}
\hline SDG & Indicator & $\mathbf{N}$ & Mean & SD & Min & $\operatorname{Max}$ \\
\hline \multirow[t]{2}{*}{1} & $\begin{array}{l}\text { Poverty headcount ratio at } \$ 1.90 \text { a day (2011 PPP) (\% of } \\
\text { population) }\end{array}$ & 166 & 14.62 & 21.97 & 0 & 81.76 \\
\hline & $\begin{array}{l}\text { Poverty rate after taxes and transfers, poverty line } 50 \% \text { (\% of } \\
\text { population) }\end{array}$ & 34 & 11.26 & 4.25 & 21 & 6 \\
\hline \multirow[t]{6}{*}{2} & Prevalence of undernourishment (\% of population) & 163 & 10.09 & 10.99 & 1.17 & 53.4 \\
\hline & Cereal yield (t/ha) & 172 & 3.25 & 2.14 & 0.04 & 11.54 \\
\hline & $\begin{array}{l}\text { Prevalence of stunting (low height-for-age) in children under } 5 \\
\text { years of age }(\%)\end{array}$ & 143 & 22.08 & 13.83 & 0 & 57.7 \\
\hline & Prevalence of wasting in children under 5 years of age (\%) & 143 & 5.97 & 4.89 & 0 & 22.7 \\
\hline & Sustainable Nitrogen Management Index (0-1) & 136 & 0.77 & 0.2 & 0.28 & 1.28 \\
\hline & Prevalence of obesity, BMI $\geq 30$ ( $\%$ of adult population) & 189 & 19.06 & 10.45 & 2.2 & 47.6 \\
\hline \multirow[t]{11}{*}{3} & Mortality rate, under-5 (per 1,000 live births) & 191 & 31.99 & 32.81 & 1.9 & 156.9 \\
\hline & Maternal mortality rate (per 100,000 live births) & 191 & 161.32 & 230.14 & 0 & 1360 \\
\hline & Neonatal mortality rate (per 1000 live births) & 191 & 13.62 & 11.35 & 0 & 48.7 \\
\hline & Physician density (per 1000 people) & 174 & 1.56 & 1.55 & 0.01 & 7.74 \\
\hline & Incidence of tuberculosis (per 100,000 people) & 191 & 120.11 & 158.66 & 0 & 852 \\
\hline & Traffic deaths rate (per 100,000 people) & 177 & 16.77 & 9,96 & 0 & 73.4 \\
\hline & Adolescent fertility rate (births per 1,000 women ages $15-19$ ) & 183 & 55,49 & 48.17 & 0.7 & 229 \\
\hline & Subjective wellbeing (average ladder score, $0-10$ ) & 152 & 5.37 & 1.16 & 2.84 & 7.59 \\
\hline & Healthy life expectancy at birth (years) & 191 & 61.54 & 8.02 & 39 & 76 \\
\hline & $\begin{array}{l}\text { Percentage of surviving infants who received } 2 \mathrm{WHO}- \\
\text { recommended vaccines }(\%)\end{array}$ & 191 & 86.13 & 14.61 & 22 & 99 \\
\hline & Daily smokers (\% of population aged $15+)^{*}$ & 34 & 19.83 & 5.72 & 10.7 & 38.9 \\
\hline \multirow[t]{6}{*}{4} & Expected years of schooling (years) & 186 & 12.87 & 2.88 & 4.1 & 20.22 \\
\hline & Literacy rate of $15-24$ year olds, both sexes (\%) & 148 & 88.34 & 16.83 & 23.52 & 100 \\
\hline & Net primary school enrolment rate $(\%)$ & 137 & 91.44 & 8.84 & 37.69 & 100 \\
\hline & Population aged $25-64$ with tertiary education $(\%)^{*}$ & 34 & 31.5 & 9.83 & 14.03 & 51.32 \\
\hline & PISA score $(0-600)^{*}$ & 60 & 468.99 & 47 & 375 & 542.67 \\
\hline & $\begin{array}{l}\text { Population aged } 25-64 \text { with upper secondary and post- } \\
\text { secondary non-tertiary educational attainment }(\%)^{\star}\end{array}$ & 34 & 17.22 & 13.27 & 0 & 56.53 \\
\hline \multirow[t]{5}{*}{5} & Proportion of seats held by women in national parliaments (\%) & 191 & 20.61 & 12.15 & 0 & 63.8 \\
\hline & $\begin{array}{l}\text { Female mean years of schooling of population aged } 25 \text { and } \\
\text { above (\% of male) }\end{array}$ & 167 & 86.18 & 20.28 & 22.61 & 134.2 \\
\hline & Female labor force participation rate (\% of male) & 121 & 72.14 & 18.48 & 14.9 & 109.76 \\
\hline & $\begin{array}{l}\text { Estimated demand for contraception that is unmet (\% of women } \\
\text { married or in union, ages 15-49) }\end{array}$ & 182 & 39.01 & 20.89 & 5.41 & 93.01 \\
\hline & 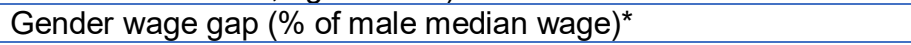 & 26 & 14.35 & 6.77 & 6.17 & 36.3 \\
\hline \multirow[t]{3}{*}{6} & Access to improved water source (\% of population) & 189 & 88.23 & 15.2 & 31.7 & 100 \\
\hline & Access to improved sanitation facilities (\% of population) & 188 & 72.35 & 29.18 & 6.7 & 100 \\
\hline & Freshwater withdrawal (\% of total renewable water resources) & 171 & 51.79 & 229.48 & 0.01 & 2075 \\
\hline \multirow[t]{4}{*}{7} & Access to electricity (\% of population) & 192 & 77.17 & 30.76 & 5.06 & 100 \\
\hline & Access to non-solid fuels (\% of population) & 191 & 64.37 & 35.14 & 0 & 99.9 \\
\hline & $\begin{array}{l}\mathrm{CO}_{2} \text { emissions from fuel combustion and electricity output } \\
\left.\text { ( } \mathrm{MtCO}_{2} / \mathrm{TWh}\right)\end{array}$ & 134 & 1.43 & 0.89 & 0.08 & 6.11 \\
\hline & $\begin{array}{l}\text { Share of renewable energy in total final energy consumption } \\
(\%)^{*}\end{array}$ & 34 & 6.11 & 16.51 & 0.7 & 84.7 \\
\hline \multirow[t]{6}{*}{8} & Unemployment rate (\% of total labor force) & 177 & 9.27 & 7.46 & 0.24 & 53.93 \\
\hline & Automated teller machines (ATMs per 100,000 adults) & 179 & 46.64 & 46.43 & 0.4 & 290.66 \\
\hline & Adjusted growth rate (\%) & 184 & -2.07 & 2.95 & 5.41 & -16.55 \\
\hline & Youth not in employment, education or training (NEET) $(\%)^{*}$ & 34 & 15.29 & 6.09 & 6.58 & 31.56 \\
\hline & Percentage of children 5-14 years old involved in child labor (\%) & 162 & 10.77 & 12.03 & 0 & 49 \\
\hline & Employment-to-Population ratio $(\%)^{*}$ & 34 & 60.14 & 10.41 & 28.73 & 78.51 \\
\hline
\end{tabular}




\begin{tabular}{|c|c|c|c|c|c|c|}
\hline SDG & Indicator & $\mathbf{N}$ & Mean & SD & Min & Max \\
\hline \multirow[t]{7}{*}{9} & Research and development expenditure ( $\%$ of GDP) & 161 & 0.65 & 0.92 & 0 & 4.04 \\
\hline & Research and development researchers (per 1000 employed) ${ }^{*}$ & 34 & 8.67 & 3.61 & 0.83 & 17.38 \\
\hline & $\begin{array}{l}\text { Logistics Performance Index: Quality of trade and transport- } \\
\text { related infrastructure }(1-5)\end{array}$ & 163 & 2.75 & 0.65 & 1.5 & 4.32 \\
\hline & Quality of overall infrastructure (1-7) & 138 & 4.11 & 1.06 & 2.1 & 6.47 \\
\hline & Mobile broadband subscriptions (per 100 inhabitants) & 142 & 34.57 & 32.81 & 0 & 149.3 \\
\hline & Proportion of the population using the internet (\%) & 187 & 43.64 & 29.48 & 0 & 98.16 \\
\hline & $\begin{array}{l}\text { Patent applications filed under the PCT in the inventor's country } \\
\text { of residence (per million population)* }\end{array}$ & 34 & 116.2 & 104.09 & 1.83 & 343.1 \\
\hline \multirow[t]{3}{*}{10} & Gini index $(0-100)$ & 146 & 39.77 & 9.32 & 24.9 & 65.77 \\
\hline & Palma ratio* & 34 & 1.26 & 0.53 & 0.82 & 3.26 \\
\hline & PISA Social Justice Index $(0-10)^{*}$ & 28 & 5.6 & 1.09 & 3.57 & 7.48 \\
\hline \multirow[t]{3}{*}{11} & $\begin{array}{l}\text { Annual mean concentration of particulate matter of less than } 2.5 \\
\text { microns of diameter }(\mathrm{PM} 2.5)\left(\mathrm{\mu g} / \mathrm{m}^{3}\right) \text { in urban areas }\end{array}$ & 186 & 18.24 & 11.24 & 4.36 & 70.13 \\
\hline & Rooms per person* & 34 & 1.69 & 0.42 & 1 & 2.5 \\
\hline & $\begin{array}{l}\text { Improved water source, piped (\% of urban population with } \\
\text { access) }\end{array}$ & 173 & 74.59 & 29.57 & 3.48 & 100 \\
\hline \multirow[t]{3}{*}{12} & $\begin{array}{l}\text { Percentage of anthropogenic wastewater that receives } \\
\text { treatment }(\%)\end{array}$ & 172 & 25.75 & 32.4 & 0 & 100 \\
\hline & Municipal solid waste (kg/year/capita) & 159 & 1.52 & 1.54 & 0.09 & 14.4 \\
\hline & Non-recycled municipal solid waste (kg/person/year) ${ }^{*}$ & 32 & 1.43 & 0.44 & 0.52 & 2.36 \\
\hline \multirow[t]{2}{*}{13} & Energy-related $\mathrm{CO}_{2}$ emissions per capita ( $\mathrm{tCO}_{2} /$ capita) & 188 & 4.63 & 6.25 & 0.02 & 44.02 \\
\hline & Climate Change Vulnerability Monitor (0-1) & 158 & 0.11 & 0.09 & 0.01 & 0.43 \\
\hline \multirow[t]{5}{*}{14} & Ocean Health Index Goal - Clean Waters (0-100) & 148 & 65.49 & 11.08 & 34.74 & 93.92 \\
\hline & Ocean Health Index Goal - Biodiversity $(0-100)$ & 148 & 83.63 & 7.4 & 64.67 & 98.26 \\
\hline & Ocean Health Index Goal - Fisheries (0-100) & 146 & 57.53 & 24.52 & 1 & 98 \\
\hline & $\begin{array}{l}\text { Marine sites of biodiversity importance that are completely } \\
\text { protected (\%) }\end{array}$ & 134 & 18.92 & 25.24 & 0 & 100 \\
\hline & Percentage of fish stocks overexploited or collapsed by EEZ (\%) & 112 & 32.12 & 25.35 & 0.02 & 95.01 \\
\hline \multirow[t]{3}{*}{15} & Red List Index of species survival $(0-1)$ & 192 & 0.86 & 0.1 & 0.4 & 0.99 \\
\hline & Annual change in forest area (\%) & 179 & 6.93 & 12.32 & 0 & 100.73 \\
\hline & $\begin{array}{l}\text { Terrestrial sites of biodiversity importance that are completely } \\
\text { protected }(\%)\end{array}$ & 188 & 18.93 & 20.72 & 0 & 100 \\
\hline \multirow[t]{7}{*}{16} & Homicides (per 100,000 people) & 192 & 8.55 & 11.25 & 0 & 90.4 \\
\hline & Prison population (per 100,000 people) & 188 & 165.77 & 131.94 & 6 & 716 \\
\hline & $\begin{array}{l}\text { Proportion of the population who feel safe walking alone at night } \\
\text { in the city or area where they live. (\%) }\end{array}$ & 156 & 61.08 & 15.35 & 13.82 & 92.31 \\
\hline & Corruption Perception Index (0-100) & 162 & 42.3 & 20.24 & 8 & 91 \\
\hline & $\begin{array}{l}\text { Proportion of children under } 5 \text { years of age whose births have } \\
\text { been registered with a civil authority, by age (\%) }\end{array}$ & 160 & 81.09 & 26.16 & 2.3 & 100 \\
\hline & Government efficiency (1-7) & 138 & 3.63 & 0.77 & 1.41 & 5.77 \\
\hline & Property rights (1-7) & 138 & 4.33 & 0.96 & 1.59 & 6.42 \\
\hline \multirow[t]{3}{*}{17} & $\begin{array}{l}\text { For high-income and all OECD DAC countries: International } \\
\text { concessional public finance, including official development } \\
\text { assistance }(\% \text { of } \mathrm{GNI})^{*}\end{array}$ & 28 & 0.41 & 0.33 & 0.10 & 1.41 \\
\hline & For all other countries: Tax revenue ( $\%$ of GDP) & 128 & 28.59 & 15.35 & 8.36 & 107.49 \\
\hline & Health, education and R\&D spending ( $\%$ of GDP) & 120 & 12.72 & 4.59 & 2.56 & 25.12 \\
\hline
\end{tabular}


Table 10 | Shapiro-Wilk test for normality on SDG Index indicators

Table describes number of observations (Obs.), Shapiro-Wilk test statistics (W), z-statistic (z), and probability of $z$ under null hypothesis of normality (Prob>z). Small values of Prob $>z$ suggest that null hypothesis of normality can be rejected.

\begin{tabular}{|c|c|c|c|c|c|}
\hline SDG & Indicator & Obs. & $\mathbf{W}$ & $\mathbf{z}$ & Prob $>z$ \\
\hline \multirow[t]{2}{*}{1} & Poverty headcount ratio at $\$ 1.90$ a day (2011 PPP) (\% of population) & 166 & 0.78450 & 7.5410 & 0.0000 \\
\hline & Poverty rate after taxes and transfers, poverty line $50 \%$ ( $\%$ of population) & 34 & 0.91427 & 2.2850 & 0.0112 \\
\hline \multirow[t]{6}{*}{2} & Prevalence of undernourishment (\% of population) & 163 & 0.83159 & 6.9370 & 0.0000 \\
\hline & Cereal yield (t/ha) & 172 & 0.93341 & 4.9440 & 0.0000 \\
\hline & $\begin{array}{l}\text { Prevalence of stunting (low height-for-age) in children under } 5 \text { years of age } \\
(\%)\end{array}$ & 143 & 0.96209 & 3.2640 & 0.0006 \\
\hline & Prevalence of wasting in children under 5 years of age (\%) & 143 & 0.88114 & 5.8480 & 0.0000 \\
\hline & Sustainable Nitrogen Management Index (0-1) & 136 & 0.98261 & 1.4010 & 0.0806 \\
\hline & Prevalence of obesity, BMI $\geq 30$ (\% of adult population) & 189 & 0.96027 & 3.9700 & 0.0000 \\
\hline \multirow[t]{11}{*}{3} & Mortality rate, under- 5 (per 1,000 live births) & 152 & 0.97661 & 2.2970 & 0.0108 \\
\hline & Maternal mortality rate (per 100,000 live births) & 191 & 0.81995 & 7.4620 & 0.0000 \\
\hline & Neonatal mortality rate (per 1000 live births) & 191 & 0.95816 & 4.1120 & 0.0000 \\
\hline & Physician density (per 1000 people) & 191 & 0.77254 & 7.9980 & 0.0000 \\
\hline & Incidence of tuberculosis (per 100,000 people) & 191 & 0.71609 & 8.5070 & 0.0000 \\
\hline & Traffic deaths rate (per 100,000 people) & 191 & 0.89680 & 6.1840 & 0.0000 \\
\hline & Adolescent fertility rate (births per 1,000 women ages $15-19$ ) & 174 & 0.86394 & 6.6020 & 0.0000 \\
\hline & Subjective wellbeing (average ladder score, $0-10$ ) & 191 & 0.73088 & 8.3840 & 0.0000 \\
\hline & Healthy life expectancy at birth (years) & 177 & 0.91889 & 5.4590 & 0.0000 \\
\hline & $\begin{array}{l}\text { Percentage of surviving infants who received } 2 \text { WHO-recommended } \\
\text { vaccines }(\%)\end{array}$ & 183 & 0.88749 & 6.2830 & 0.0000 \\
\hline & Daily smokers (\% of population aged $15+$ ) & 34 & 0.93821 & 1.6020 & 0.0545 \\
\hline \multirow[t]{6}{*}{4} & Expected years of schooling (years) & 186 & 0.99430 & -0.5170 & 0.6976 \\
\hline & Literacy rate of $15-24$ year olds, both sexes (\%) & 148 & 0.72154 & 7.8540 & 0.0000 \\
\hline & Net primary school enrolment rate (\%) & 137 & 0.76186 & 7.3200 & 0.0000 \\
\hline & Population aged 25-64 with tertiary education (\%) & 34 & 0.96069 & 0.6600 & 0.2546 \\
\hline & PISA score $(0-600)$ & 60 & 0.92680 & 2.9770 & 0.0015 \\
\hline & $\begin{array}{l}\text { Population aged } 25-64 \text { with upper secondary and post-secondary non- } \\
\text { tertiary educational attainment (\%) }\end{array}$ & 34 & 0.80958 & 3.9480 & 0.0000 \\
\hline \multirow[t]{5}{*}{$\overline{5}$} & Proportion of seats held by women in national parliaments (\%) & 191 & 0.97073 & 3.2920 & 0.0005 \\
\hline & $\begin{array}{l}\text { Female mean years of schooling of population aged } 25 \text { and above (\% of } \\
\text { male) }\end{array}$ & 167 & 0.91455 & 5.4470 & 0.0000 \\
\hline & Female labor force participation rate (\% of male) & 121 & 0.91134 & 4.8210 & 0.0000 \\
\hline & $\begin{array}{l}\text { Estimated demand for contraception that is unmet (\% of women married or } \\
\text { in union, ages 15-49) }\end{array}$ & 182 & 0.93720 & 4.9360 & 0.0000 \\
\hline & Gender wage gap (\% of male median wage) & 26 & 0.88195 & 2.4930 & 0.0063 \\
\hline \multirow[t]{3}{*}{6} & Access to improved water source (\% of population) & 189 & 0.78815 & 7.8100 & 0.0000 \\
\hline & Access to improved sanitation facilities (\% of population) & 188 & 0.85443 & 6.9370 & 0.0000 \\
\hline & Freshwater withdrawal (\% of total renewable water resources) & 171 & 0.20530 & 10.5900 & 0.0000 \\
\hline \multirow[t]{4}{*}{7} & Access to electricity (\% of population) & 192 & 0.86324 & 6.8430 & 0.0000 \\
\hline & Access to non-solid fuels ( $\%$ of population) & 191 & 0.87178 & 6.6830 & 0.0000 \\
\hline & $\mathrm{CO}_{2}$ emissions from fuel combustion and electricity output ( $\left.\mathrm{MtCO}_{2} / \mathrm{TWh}\right)$ & 134 & 0.78461 & 7.0430 & 0.0000 \\
\hline & Share of renewable energy in total final energy consumption (\%) & 34 & 0.73235 & 4.6570 & 0.0000 \\
\hline \multirow[t]{6}{*}{8} & Unemployment rate (\% of total labor force) & 179 & 0.82282 & 7.2720 & 0.0000 \\
\hline & Automated teller machines (ATMs per 100,000 adults) & 184 & 0.89432 & 6.1530 & 0.0000 \\
\hline & Adjusted growth rate $(\%)$ & 34 & 0.90952 & 2.3970 & 0.0083 \\
\hline & Youth not in employment, education or training (NEET) (\%) & 162 & 0.89794 & 5.7830 & 0.0000 \\
\hline & Percentage of children 5-14 years old involved in child labor (\%) & 177 & 0.79538 & 7.5740 & 0.0000 \\
\hline & Employment-to-Population ratio (\%) & 34 & 0.96194 & 0.5930 & 0.2767 \\
\hline \multirow[t]{7}{*}{9} & Research and development expenditure (\% of GDP) & 137 & 0.78399 & 7.1000 & 0.0000 \\
\hline & Research and development researchers (per 1000 employed) & 34 & 0.96114 & 0.6360 & 0.2624 \\
\hline & $\begin{array}{l}\text { Logistics Performance Index: Quality of trade and transport-related } \\
\text { infrastructure (1-5) }\end{array}$ & 163 & 0.93199 & 4.8720 & 0.0000 \\
\hline & Quality of overall infrastructure (1-7) & 138 & 0.97797 & 1.9640 & 0.0248 \\
\hline & Mobile broadband subscriptions (per 100 inhabitants) & 142 & 0.89209 & 5.6140 & 0.0000 \\
\hline & Proportion of the population using the internet (\%) & 187 & 0.93701 & 5.0030 & 0.0000 \\
\hline & $\begin{array}{l}\text { Patent applications filed under the PCT in the inventor's country of } \\
\text { residence (per million population) }\end{array}$ & 34 & 0.88846 & 2.8330 & 0.0023 \\
\hline \multirow[t]{3}{*}{10} & Gini index $(0-100)$ & 146 & 0.95441 & 3.7250 & 0.0001 \\
\hline & Palma ratio & 34 & 0.67817 & 5.0410 & 0.0000 \\
\hline & PISA Social Justice Index (0-10) & 28 & 0.96277 & 0.2410 & 0.4047 \\
\hline \multirow[t]{3}{*}{11} & $\begin{array}{l}\text { Annual mean concentration of particulate matter of less than } 2.5 \text { microns } \\
\text { of diameter (PM2.5) }\left(\mu \mathrm{g} / \mathrm{m}^{3}\right) \text { in urban areas }\end{array}$ & 186 & 0.84836 & 7.0050 & 0.0000 \\
\hline & Rooms per person & 173 & 0.84810 & 6.8400 & 0.0000 \\
\hline & Improved water source, piped (\% of urban population with access) & 34 & 0.96590 & 0.3640 & 0.3580 \\
\hline
\end{tabular}




\begin{tabular}{|c|c|c|c|c|c|}
\hline SDG & Indicator & Obs. & $\mathbf{W}$ & $\mathbf{z}$ & Prob>z \\
\hline \multirow[t]{3}{*}{12} & Percentage of anthropogenic wastewater that receives treatment (\%) & 172 & 0.79920 & 7.4640 & 0.0000 \\
\hline & Municipal solid waste (kg/year/capita) & 159 & 0.64828 & 8.5540 & 0.0000 \\
\hline & Non-recycled municipal solid waste (kg/person/year) & 32 & 0.97445 & -0.3310 & 0.6298 \\
\hline \multirow[t]{2}{*}{13} & Energy-related $\mathrm{CO}_{2}$ emissions per capita $\left(\mathrm{tCO}_{2} /\right.$ capita) & 188 & 0.68632 & 8.6980 & 0.0000 \\
\hline & Climate Change Vulnerability Monitor (0-1) & 158 & 0.83363 & 6.8370 & 0.0000 \\
\hline \multirow[t]{5}{*}{14} & Ocean Health Index Goal - Clean Waters $(0-100)$ & 148 & 0.98973 & 0.3780 & 0.3527 \\
\hline & Ocean Health Index Goal - Biodiversity $(0-100)$ & 148 & 0.94792 & 4.0550 & 0.0000 \\
\hline & Ocean Health Index Goal - Fisheries (0-100) & 146 & 0.94123 & 4.3000 & 0.0000 \\
\hline & Marine sites of biodiversity importance that are completely protected (\%) & 134 & 0.86514 & 5.9870 & 0.0000 \\
\hline & Percentage of fish stocks overexploited or collapsed by EEZ (\%) & 112 & 0.92314 & 4.3370 & 0.0000 \\
\hline \multirow[t]{3}{*}{15} & Red List Index of species survival $(0-1)$ & 192 & 0.93871 & 5.0000 & 0.0000 \\
\hline & Annual change in forest area (\%) & 179 & 0.42110 & 9.9800 & 0.0000 \\
\hline & $\begin{array}{l}\text { Terrestrial sites of biodiversity importance that are completely protected } \\
(\%)\end{array}$ & 188 & 0.88761 & 6.3430 & 0.0000 \\
\hline \multirow[t]{7}{*}{16} & Homicides (per 100,000 people) & 192 & 0.66789 & 8.8800 & 0.0000 \\
\hline & Prison population (per 100,000 people) & 188 & 0.83573 & 7.2140 & 0.0000 \\
\hline & $\begin{array}{l}\text { Proportion of the population who feel safe walking alone at night in the city } \\
\text { or area where they live. }(\%)\end{array}$ & 156 & 0.98126 & 1.8480 & 0.0323 \\
\hline & Corruption Perception Index $(0-100)$ & 162 & 0.93084 & 4.8970 & 0.0000 \\
\hline & $\begin{array}{l}\text { Proportion of children under } 5 \text { years of age whose births have been } \\
\text { registered with a civil authority, by age (\%) }\end{array}$ & 160 & 0.79250 & 7.3690 & 0.0000 \\
\hline & Government efficiency (1-7) & 138 & 0.97163 & 2.5350 & 0.0056 \\
\hline & Property rights (1-7) & 138 & 0.97133 & 2.5590 & 0.0053 \\
\hline \multirow[t]{3}{*}{17} & $\begin{array}{l}\text { For high-income and all OECD DAC countries: International concessional } \\
\text { public finance, including official development assistance (\% of GNI) }\end{array}$ & 28 & 0.83507 & 3.3060 & 0.0005 \\
\hline & For all other countries: Tax revenue ( $\%$ of GDP) & 128 & 0.77048 & 7.0810 & 0.0000 \\
\hline & Health, education and R\&D spending (\% of GDP) & 120 & 0.99043 & -0.1840 & 0.5729 \\
\hline
\end{tabular}


Table 11 | Indicator thresholds used in the SDG Index and Dashboards Upper bounds (Best $=100)$ and lower bounds $($ Worst $=0)$ used in scaling indicators from 0 to 100, and thresholds for SDG achievement (green), significant (yellow), and major (red) challenges used in constructing the SDG Dashboards.

\begin{tabular}{|c|c|c|c|c|c|c|}
\hline SDG & Indicator & $\begin{array}{c}\text { Best } \\
(=100)\end{array}$ & Green & Yellow & Red & $\begin{array}{l}\text { Worst } \\
(=0)\end{array}$ \\
\hline \multirow[t]{2}{*}{1} & $\begin{array}{l}\text { Poverty headcount ratio at } \$ 1.90 \text { a day (2011 PPP) (\% of } \\
\text { population) }\end{array}$ & $0 \%$ & $<2 \%$ & $2 \%<=$ value $<=12.7 \%$ & $>12.7 \%$ & $68.70 \%$ \\
\hline & $\begin{array}{l}\text { Poverty rate after taxes and transfers, Poverty line } 50 \% \text { (\% of } \\
\text { population) }\end{array}$ & $0 \%$ & $<10 \%$ & $10 \%<=$ value $<=15 \%$ & $>15 \%$ & $21 \%$ \\
\hline \multirow[t]{6}{*}{2} & Prevalence of undernourishment (\% of population) & $0 \%$ & $<7.5 \%$ & $7.5 \%<=$ value $<=15 \%$ & $>15 \%$ & $41.60 \%$ \\
\hline & Cereal yield (t/ha) & 9.3 & $>2.5$ & $1.5<=$ value $<=2.5$ & $<1.5$ & 0.4 \\
\hline & $\begin{array}{l}\text { Prevalence of stunting (low height-for-age) in children under } 5 \\
\text { years of age }(\%)\end{array}$ & $0 \%$ & $<7.5 \%$ & $7.5 \%<=$ value $<=15 \%$ & $>15 \%$ & $49.50 \%$ \\
\hline & Prevalence of wasting in children under 5 years of age $(\%)$ & $0 \%$ & $<5 \%$ & $5 \%<=$ value $<=10 \%$ & $>10 \%$ & $18.90 \%$ \\
\hline & Sustainable Nitrogen Management Index $(0-1)$ & 0 & $<0.3$ & $0.3<=$ value $<=0.7$ & $>0.7$ & 1.1 \\
\hline & Prevalence of obesity, BMI $\geq 30$ ( $\%$ of adult population) & $0 \%$ & $<10 \%$ & $10 \%<=$ value $<=25 \%$ & $>25 \%$ & $42.30 \%$ \\
\hline \multirow[t]{11}{*}{3} & Mortality rate, under-5 (per 1,000 live births) & 0 & $<25$ & $25<=$ value $<=50$ & $>50$ & 120.4 \\
\hline & Maternal mortality rate (per 100,000 live births) & 0 & $<70$ & $70<=$ value $<=140$ & $>140$ & 789 \\
\hline & Neonatal mortality rate (per 1000 live births) & 0 & $<12$ & $12<=$ value $<=18$ & $>18$ & 39.7 \\
\hline & Physician density (per 1000 people) & 6.3 & $>3$ & $1<=$ value $<=3$ & $<1$ & 0 \\
\hline & Incidence of tuberculosis (per 100,000 people) & 0 & $<10$ & $10<=$ value $<=75$ & $>75$ & 561 \\
\hline & Traffic deaths rate (per 100,000 people) & 2.1 & $<8.4$ & $8.4<=$ value $<=16.8$ & $>16.8$ & 33.2 \\
\hline & Adolescent fertility rate (births per 1,000 women ages $15-19)$ & 0 & $<25$ & $25<=$ value $<=50$ & $>50$ & 176 \\
\hline & Subjective Wellbeing (average ladder score, $0-10$ ) & 10 & $>6$ & $5<=$ value $<=6$ & $<5$ & 3.3 \\
\hline & Healthy Life Expectancy at birth (years) & 74.2 & $>65$ & $60<=$ value $<=65$ & $<60$ & 44 \\
\hline & $\begin{array}{l}\text { Percentage of surviving infants who received } 2 \mathrm{WHO}- \\
\text { recommended vaccines }\end{array}$ & $100 \%$ & $>90 \%$ & $80 \%<=$ value $<=90 \%$ & $<80 \%$ & $46 \%$ \\
\hline & Daily smokers (\% of population aged $15+$ ) & $12.10 \%$ & $<20 \%$ & $20 \%<=$ value $<=25 \%$ & $>25 \%$ & $38.90 \%$ \\
\hline \multirow[t]{6}{*}{4} & Expected years of schooling (years) & 19.1 & $>12$ & $10<=$ value $<=12$ & $<10$ & 7.2 \\
\hline & Literacy rate of $15-24$ year olds, both sexes (\%) & $100 \%$ & $>95 \%$ & $85 \%<=$ value $<=95 \%$ & $<85 \%$ & $39.30 \%$ \\
\hline & Net primary enrolment rate $(\%)$ & $100 \%$ & $>98 \%$ & $90 \%<=$ value $<=98 \%$ & $<90 \%$ & $68.70 \%$ \\
\hline & Population aged $25-64$ with tertiary education (\%) & $45.40 \%$ & $>25 \%$ & $15 \%<=$ value $<=25 \%$ & $<15 \%$ & $14 \%$ \\
\hline & PISA score $(0-600)$ & 600 & $>493$ & $400<=$ value $<=493$ & $<400$ & 382.7 \\
\hline & $\begin{array}{l}\text { Population aged } 25-64 \text { with upper secondary and post-secondary } \\
\text { non-tertiary educational attainment (\%) }\end{array}$ & $100 \%$ & $>85 \%$ & $70 \%<=$ value $<=85 \%$ & $<70 \%$ & $0 \%$ \\
\hline \multirow[t]{5}{*}{5} & Proportion of seats held by women in national parliaments (\%) & $50 \%$ & $>40 \%$ & $20 \%<=$ value $<=40 \%$ & $<20 \%$ & $0 \%$ \\
\hline & $\begin{array}{l}\text { Female years of schooling of population aged } 25 \text { and above (\% } \\
\text { male) }\end{array}$ & $100 \%$ & $>95 \%$ & $75 \%<=$ value $<=95 \%$ & $<75 \%$ & 40.5 \\
\hline & Female labor force participation rate (\% male) & $100 \%$ & $>70 \%$ & $50 \%<=$ value $<=70 \%$ & $<50 \%$ & $22.50 \%$ \\
\hline & $\begin{array}{l}\text { Estimated demand for contraception that is unmet }(\% \text { of women } \\
\text { married or in union, ages } 15-49)\end{array}$ & $0 \%$ & $<20 \%$ & $20 \%<=$ value $<=50 \%$ & $>50 \%$ & $82.90 \%$ \\
\hline & Gender wage gap (Total, $\%$ of male median wage) & $0 \%$ & $<7.5 \%$ & $7.5 \%<=$ value $<=15 \%$ & $>15 \%$ & $36.30 \%$ \\
\hline \multirow[t]{3}{*}{6} & Access to improved water source (\% of population) & $100 \%$ & $>98 \%$ & $80 \%<=$ value $<=98 \%$ & $<80 \%$ & $50.80 \%$ \\
\hline & Access to improved sanitation facilities (\% of population) & $100 \%$ & $>95 \%$ & $75 \%<=$ value $<=95 \%$ & $<75 \%$ & $12.10 \%$ \\
\hline & Freshwater withdrawal as $\%$ of total renewable water resources & $0 \%$ & $<20 \%$ & $20 \%<=$ value $<=40 \%$ & $>40 \%$ & $374.10 \%$ \\
\hline \multirow[t]{4}{*}{7} & Access to electricity (\% of population) & $100 \%$ & $>98 \%$ & $80 \%<=$ value $<=98 \%$ & $<80 \%$ & $9.80 \%$ \\
\hline & Access to non-solid fuels (\% of population) & $100 \%$ & $>85 \%$ & $50 \%<=$ value $<=85 \%$ & $<50 \%$ & $5 \%$ \\
\hline & $\begin{array}{l}\text { CO2 emissions from fuel combustion / electricity output } \\
\text { (MtCO2/TWh) }\end{array}$ & 0 & $<1$ & $1<=$ value $<=1.5$ & $>1.5$ & 3.7 \\
\hline & Share of renewable energy in total final energy consumption (\%) & $47 \%$ & $>20 \%$ & $10 \%<=$ value $<=20 \%$ & $<10 \%$ & $0.70 \%$ \\
\hline \multirow[t]{6}{*}{8} & Unemployment rate ( $\%$ of total labor force) & $0.80 \%$ & $<5 \%$ & $5 \%<=$ value $<=10 \%$ & $>10 \%$ & $30.10 \%$ \\
\hline & Automated teller machines (ATMs per 100,000 adults) & 217.8 & $>20$ & $10<=$ value $<=20$ & $<10$ & 1 \\
\hline & Adjusted Growth (\%) & $3.70 \%$ & $>0 \%$ & $-2 \%<=$ value $<=0 \%$ & $<-2 \%$ & $-7.30 \%$ \\
\hline & Youth not in employment, education or training (NEET) & $8.30 \%$ & $<10 \%$ & $10 \%<=$ value $<=15 \%$ & $>15 \%$ & $31.60 \%$ \\
\hline & Percentage of children 5-14 years old involved in child labor & $0 \%$ & $<2 \%$ & $2 \%<=$ value $<=10 \%$ & $>10 \%$ & $39.20 \%$ \\
\hline & Employment-to-Population ratio (\%) & $73.60 \%$ & $>60 \%$ & $50 \%<=$ value $<=60 \%$ & $<50 \%$ & $28.70 \%$ \\
\hline \multirow[t]{7}{*}{9} & Research and development expenditure ( $\%$ of GDP) & $3.70 \%$ & $>1.5 \%$ & $1 \%<=$ value $<=1.5 \%$ & $<1 \%$ & $0 \%$ \\
\hline & Research and development researchers (per 1000 employed) & 15 & $>8$ & $7<=$ value $<=8$ & $<7$ & 0.8 \\
\hline & $\begin{array}{l}\text { Logistics Performance Index: Quality of trade and transport-related } \\
\text { infrastructure }(1-5)\end{array}$ & 5 & $>3$ & $2<=$ value $<=3$ & $<2$ & 1.8 \\
\hline & Quality of overall infrastructure (1-7) & 7 & $>4.5$ & $3<=$ value $<=4.5$ & $<3$ & 2.4 \\
\hline & Mobile broadband subscriptions (per 100 inhabitants) & $100 \%$ & $>75 \%$ & $50 \%<=$ value $<=75 \%$ & $<50 \%$ & $0 \%$ \\
\hline & Proportion of the population using the internet (\%) & $100 \%$ & $>80 \%$ & $50 \%<=$ value $<=80 \%$ & $<50 \%$ & $1.60 \%$ \\
\hline & $\begin{array}{l}\text { Patent applications filed under the PCT in the inventor's country of } \\
\text { residence (per million population) }\end{array}$ & 305.3 & $>50$ & $100<=$ value $<=50$ & $<100$ & 1.8 \\
\hline \multirow[t]{3}{*}{10} & Gini index $(0-100)$ & 25.4 & $<30$ & $30<=$ value $<=40$ & $>40$ & 63.1 \\
\hline & Palma ratio & 0.85 & $<1$ & $1<=$ value $<=1.2$ & $>1.2$ & 3.3 \\
\hline & PISA Social Justice Index (0-10) & 10 & $>5.6$ & $4<=$ value $<=5.6$ & $<4$ & 3.6 \\
\hline \multirow[t]{3}{*}{11} & $\begin{array}{l}\text { Annual mean concentration of particulate matter of less than } 2.5 \\
\text { microns of diameter (PM2.5) }(\mu \mathrm{g} / \mathrm{m} 3) \text { in urban areas }\end{array}$ & 0 & $<10$ & $10<=$ value $<=20$ & $>20$ & 48.4 \\
\hline & Rooms per person & 2.4 & $>1.5$ & $1.1<=$ value $<=1.5$ & $<1.1$ & 1 \\
\hline & Improved water source, piped (\% of urban population with access) & $100 \%$ & $>98 \%$ & $75 \%<=$ value $<=98 \%$ & $<75 \%$ & $6.10 \%$ \\
\hline \multirow[t]{3}{*}{12} & $\begin{array}{l}\text { Percentage of anthropogenic wastewater that receives treatment } \\
(\%)\end{array}$ & $100 \%$ & $>50 \%$ & $15 \%<=$ value $<=50 \%$ & $<15 \%$ & $0 \%$ \\
\hline & Municipal Solid Waste (kg/year/capita) & 0.1 & $<1$ & $1<=$ value $<=2$ & $>2$ & 5.4 \\
\hline & $\begin{array}{l}\text { Non-Recycled Municipal Solid Waste (MSW in kg/person/year } \\
\text { times recycling rate) }\end{array}$ & 0.7 & $<1$ & $1<=$ value $<=1.5$ & $>1.5$ & 2.4 \\
\hline
\end{tabular}


On metrics and financing for the Sustainable Development Goals

\begin{tabular}{|c|c|c|c|c|c|c|}
\hline SDG & Indicator & $\begin{array}{c}\text { Best } \\
(=100)\end{array}$ & Green & Yellow & Red & $\begin{array}{c}\text { Worst } \\
(=0)\end{array}$ \\
\hline \multirow[t]{2}{*}{13} & Energy-related $\mathrm{CO} 2$ emissions per capita (tCO2/capita) & 0 & $<2$ & $2<=$ value $<=4$ & $>4$ & 20.9 \\
\hline & Climate Change Vulnerability Monitor $(0-1)$ & 0 & $<0.1$ & $0.1<=$ value $<=0.2$ & $>0.2$ & 0.4 \\
\hline \multirow[t]{5}{*}{14} & Ocean Health Index Goal - Clean Waters (0-100) & 100 & $>70$ & $60<=$ value $<=70$ & $<60$ & 44.1 \\
\hline & Ocean Health Index Goal - Biodiversity $(0-100)$ & 100 & $>90$ & $80<=$ value $<=90$ & $<80$ & 66.4 \\
\hline & Ocean Health Index Goal - Fisheries $(0-100)$ & 100 & $>70$ & $60<=$ value $<=70$ & $<60$ & 2 \\
\hline & $\begin{array}{l}\text { Marine sites of biodiversity importance that are completely } \\
\text { protected (\%) }\end{array}$ & $100 \%$ & $>50 \%$ & $10 \%<=$ value $<=50 \%$ & $<10 \%$ & $0 \%$ \\
\hline & Percentage of Fish Stocks overexploited or collapsed by EEZ (\%) & 0 & $<25$ & $25<=$ value $<=50$ & $>50$ & 91.7 \\
\hline \multirow[t]{3}{*}{15} & Red List Index of species survival (0-1) & 1 & $>0.9$ & $0.8<=$ value $<=0.9$ & $<0.8$ & 0.7 \\
\hline & Annual change in forest area (\%) & 0.1 & $<0$ & $0<=$ value $<=-2$ & $>-2$ & 31 \\
\hline & $\begin{array}{l}\text { Terrestrial sites of biodiversity importance that are completely } \\
\text { protected }(\%)\end{array}$ & $100 \%$ & $>50 \%$ & $10 \%<=$ value $<=50 \%$ & $<10 \%$ & $0 \%$ \\
\hline \multirow[t]{7}{*}{16} & Homicides (per 100,000 people) & 0 & $<1.5$ & $1.5<=$ value $<=3$ & $>3$ & 39.9 \\
\hline & Prison population (per 100,000 people) & 18 & $<100$ & $100<=$ value $<=200$ & $>200$ & 510 \\
\hline & $\begin{array}{l}\text { Proportion of the population who feel safe walking alone at night in } \\
\text { the city or area where they live. (\%) }\end{array}$ & $100 \%$ & $>80 \%$ & $50 \%<=$ value $<=80 \%$ & $<50 \%$ & $34.80 \%$ \\
\hline & Corruption Perception Index $(0-100)$ & 100 & $>60$ & $40<=$ value $<=60$ & $<40$ & 15 \\
\hline & $\begin{array}{l}\text { Proportion of children under } 5 \text { years of age whose births have } \\
\text { been registered with a civil authority, by age (\%) }\end{array}$ & $100 \%$ & $>98 \%$ & $75 \%<=$ value $<=98 \%$ & $<75 \%$ & $10.30 \%$ \\
\hline & Government Efficiency (1-7) & 7 & $>4.5$ & $3<=$ value $<=4.5$ & $<3$ & 2.5 \\
\hline & Property Rights (1-7) & 7 & $>4.5$ & $3<=$ value $<=4.5$ & $<3$ & 2.6 \\
\hline \multirow[t]{3}{*}{17} & $\begin{array}{l}\text { For high-income and all OECD DAC countries: International } \\
\text { concessional public finance, including official development } \\
\text { assistance (\% of GNI) }\end{array}$ & $1 \%$ & $>0.7 \%$ & $0.35 \%<=$ value $<=0.7 \%$ & $<0.35 \%$ & $0.10 \%$ \\
\hline & For all other countries: Tax revenue ( $\%$ of GDP) & $84.60 \%$ & $>25 \%$ & $15 \%<=$ value $<=25 \%$ & $<15 \%$ & $11 \%$ \\
\hline & Health, Education and R\&D spending (\% of GDP) & $23 \%$ & $>16 \%$ & $8 \%<=$ value $<=16 \%$ & $<8 \%$ & $5.10 \%$ \\
\hline
\end{tabular}




\section{2 - National baselines for the SDGs}

Table 12 | Countries not included in the SDG Index and Dashboards

Table lists countries not included due to insufficient data availability. Missing values denotes the share of the 63 indicators used in the SDG Index and Dashboards for which the country lacks data.

\begin{tabular}{|l|c|}
\multicolumn{1}{|c|}{ Country } & $\begin{array}{c}\text { Missing } \\
\text { Values (\%) }\end{array}$ \\
\hline Andorra & 56 \\
\hline $\begin{array}{l}\text { Antigua and } \\
\text { Barbuda }\end{array}$ & 44 \\
\hline Bahamas, The & 37 \\
\hline Bahrain & 21 \\
\hline Barbados & 31 \\
\hline Belize & 26 \\
\hline $\begin{array}{l}\text { Brunei } \\
\text { Darussalam }\end{array}$ & 40 \\
\hline Comoros & 27 \\
\hline Cuba & 24 \\
\hline Djibouti & 27 \\
\hline Dominica & 48 \\
\hline $\begin{array}{l}\text { Equatorial } \\
\text { Guinea }\end{array}$ & 27 \\
\hline Eritrea & Fiji \\
\hline Grenada & 27 \\
\hline
\end{tabular}

\begin{tabular}{|l|c|}
\hline \multicolumn{1}{|c|}{ Country } & $\begin{array}{c}\text { Missing } \\
\text { Values (\%) }\end{array}$ \\
\hline Guinea-Bissau & 23 \\
\hline Kiribati & 40 \\
\hline $\begin{array}{l}\text { Korea, Dem. } \\
\text { Rep. }\end{array}$ & 40 \\
\hline Libya & 27 \\
\hline Liechtenstein & 63 \\
\hline Maldives & 24 \\
\hline $\begin{array}{l}\text { Marshall } \\
\text { Islands }\end{array}$ & 47 \\
\hline $\begin{array}{l}\text { Micronesia, } \\
\text { Fed. Sts. }\end{array}$ & 45 \\
\hline Monaco & 55 \\
\hline Nauru & 47 \\
\hline Palau & 29 \\
\hline $\begin{array}{l}\text { Papua New } \\
\text { Guinea }\end{array}$ & 40 \\
\hline Samoa & \\
\hline San Marino & $\begin{array}{l}\text { Sao Tome and } \\
\text { Principe }\end{array}$ \\
\hline
\end{tabular}

\begin{tabular}{|l|c|}
\multicolumn{1}{|c|}{ Country } & $\begin{array}{c}\text { Missing } \\
\text { Values }(\%)\end{array}$ \\
\hline Seychelles & 24 \\
\hline $\begin{array}{l}\text { Solomon } \\
\text { Islands }\end{array}$ & 32 \\
\hline Somalia & 37 \\
\hline South Sudan & 37 \\
\hline $\begin{array}{l}\text { St. Kitts and } \\
\text { Nevis }\end{array}$ & 50 \\
\hline St. Lucia & 37 \\
\hline $\begin{array}{l}\text { St. Vincent and } \\
\text { the Grenadines }\end{array}$ & 47 \\
\hline $\begin{array}{l}\text { Syrian Arab } \\
\text { Republic }\end{array}$ & 21 \\
\hline Timor-Leste & 23 \\
\hline Tonga & 37 \\
\hline Turkmenistan & 29 \\
\hline Tuvalu & 56 \\
\hline Uzbekistan & 23 \\
\hline Vanuatu & 31 \\
\hline
\end{tabular}


On metrics and financing for the Sustainable Development Goals

Table 13 | SDG Index rankings obtained using alternative scaling methods

Comparison of index ranks obtained using different methods to set the lower $(=0)$ bound.

\begin{tabular}{|c|c|c|c|c|c|c|}
\hline \multirow[t]{2}{*}{ Country } & \multicolumn{2}{|c|}{$\begin{array}{l}\text { SDG Index: Worst }(=0) \\
\text { - data truncated at } 2.5 \text { th } \\
\text { percentile } \\
\end{array}$} & \multicolumn{2}{|c|}{$\begin{array}{c}\text { Worst }(=0) \\
\text { - data truncated at } 5 \text { th } \\
\text { percentile }\end{array}$} & \multicolumn{2}{|c|}{$\begin{array}{c}\text { Worst }(=0) \\
\text { - average of } 5 \text { worst per- } \\
\text { formers }\end{array}$} \\
\hline & & & & & & \\
\hline Sweden & 1 & 84.5 & 0 & -2.3 & 0 & 0.6 \\
\hline Denmark & 2 & 83.9 & 0 & -2.7 & 0 & 0.8 \\
\hline Norway & 3 & 82.3 & 0 & -2.4 & 0 & 0.5 \\
\hline Finland & 4 & 81.0 & -1 & -2.8 & 0 & 0.8 \\
\hline Switzerland & 5 & 80.9 & 1 & -2.3 & 0 & 0.9 \\
\hline Germany & 6 & 80.5 & 0 & -2.5 & 0 & 1.0 \\
\hline Austria & 7 & 79.1 & -1 & -2.3 & 0 & 1.4 \\
\hline Netherlands & 8 & 78.9 & -1 & -2.8 & 0 & 1.1 \\
\hline Iceland & 9 & 78.4 & 2 & -0.8 & -1 & 1.3 \\
\hline United Kingdom & 10 & 78.1 & -1 & -2.5 & -1 & 1.1 \\
\hline France & 11 & 77.9 & -1 & -2.4 & -1 & 0.7 \\
\hline Belgium & 12 & 77.4 & -2 & -3.0 & -1 & 1.1 \\
\hline Canada & 13 & 76.8 & -3 & -3.0 & -3 & 1.0 \\
\hline Ireland & 14 & 76.7 & -3 & -3.0 & 0 & 1.3 \\
\hline Czech Republic & 15 & 76.7 & 0 & -2.5 & 0 & 1.3 \\
\hline Luxembourg & 16 & 76.7 & 6 & -0.7 & 7 & 3.1 \\
\hline Slovenia & 17 & 76.6 & 4 & -2.1 & 0 & 0.8 \\
\hline Japan & 18 & 75.0 & 0 & -2.3 & 0 & 1.5 \\
\hline Singapore & 19 & 74.6 & 0 & -1.9 & -2 & 1.4 \\
\hline Australia & 20 & 74.5 & -1 & -3.3 & 0 & 1.5 \\
\hline Estonia & 21 & 74.5 & 1 & -3.1 & 2 & 1.6 \\
\hline New Zealand & 22 & 74.0 & 0 & -3.0 & 0 & 1.2 \\
\hline Belarus & 23 & 73.5 & -1 & -3.1 & -5 & 0.8 \\
\hline Hungary & 24 & 73.4 & 1 & -2.6 & 1 & 1.6 \\
\hline United States & 25 & 72.7 & -8 & -3.8 & -1 & 1.6 \\
\hline Slovak Republic & 26 & 72.7 & 1 & -2.3 & -3 & 1.4 \\
\hline South Korea & 27 & 72.7 & 1 & -2.7 & 3 & 1.9 \\
\hline Latvia & 28 & 72.5 & 1 & -2.9 & -2 & 1.3 \\
\hline Israel & 29 & 72.3 & -7 & -4.0 & 2 & 2.0 \\
\hline Spain & 30 & 72.2 & 0 & -3.1 & -1 & 1.4 \\
\hline Lithuania & 31 & 72.1 & 2 & -3.0 & -1 & 1.5 \\
\hline Malta & 32 & 72.0 & 0 & -2.9 & 7 & 2.6 \\
\hline Bulgaria & 33 & 71.8 & 5 & -2.5 & 0 & 1.6 \\
\hline Portugal & 34 & 71.5 & -1 & -3.0 & 0 & 1.8 \\
\hline Italy & 35 & 70.9 & 1 & -2.4 & 0 & 1.9 \\
\hline Croatia & 36 & 70.7 & 5 & -1.7 & 0 & 1.8 \\
\hline Greece & 37 & 69.9 & 0 & -2.4 & 0 & 2.1 \\
\hline Poland & 38 & 69.8 & 0 & -2.4 & 0 & 1.7 \\
\hline Serbia & 39 & 68.3 & 0 & -1.1 & 0 & 2.7 \\
\hline Uruguay & 40 & 68.0 & -2 & -3.3 & -1 & 1.3 \\
\hline Romania & 41 & 67.5 & 1 & -2.2 & 1 & 1.8 \\
\hline Chile & 42 & 67.2 & -4 & -3.1 & -3 & 1.8 \\
\hline Argentina & 43 & 66.8 & -5 & -3.0 & -3 & 1.8 \\
\hline Moldova & 44 & 66.6 & 0 & -2.4 & -6 & 1.3 \\
\hline Cyprus & 45 & 66.5 & 4 & -1.4 & 3 & 2.7 \\
\hline Ukraine & 46 & 66.4 & 1 & -2.3 & -3 & 1.6 \\
\hline Russian Federation & 47 & 66.4 & -2 & -3.1 & 0 & 1.9 \\
\hline Turkey & 48 & 66.1 & 5 & -1.6 & 4 & 2.9 \\
\hline Qatar & 49 & 65.8 & 2 & -1.9 & 6 & 3.3 \\
\hline Armenia & 50 & 65.4 & 0 & -2.3 & -1 & 2.1 \\
\hline Tunisia & 51 & 65.1 & 0 & -2.1 & 3 & 3.0 \\
\hline Brazil & 52 & 64.4 & -4 & -3.7 & 0 & 1.6 \\
\hline Costa Rica & 53 & 64.2 & 1 & -2.9 & -4 & 1.4 \\
\hline Kazakhstan & 54 & 63.9 & -5 & -4.0 & -4 & 1.4 \\
\hline United Arab Emirates & 55 & 63.6 & -3 & -3.3 & 1 & 2.2 \\
\hline Mexico & 56 & 63.4 & -1 & -3.0 & 0 & 2.3 \\
\hline
\end{tabular}




\begin{tabular}{|c|c|c|c|c|c|c|}
\hline \multirow[t]{2}{*}{ Country } & \multicolumn{2}{|c|}{$\begin{array}{l}\text { SDG Index: Worst }(=0) \\
\text { - data truncated at } 2.5 \text { th } \\
\text { percentile }\end{array}$} & \multicolumn{2}{|c|}{$\begin{array}{c}\text { Worst }(=0) \\
\text { - data truncated at } 5 \text { th } \\
\text { percentile }\end{array}$} & \multicolumn{2}{|c|}{$\begin{array}{l}\text { Worst }(=0) \\
\text { - average of } 5 \text { worst per- } \\
\text { formers }\end{array}$} \\
\hline & Rank & Score & Rank diff. & Score diff. & Rank diff. & Score diff. \\
\hline Georgia & 57 & 63.3 & 3 & -2.3 & -3 & 1.8 \\
\hline Macedonia, FYR & 58 & 62.8 & 3 & -1.8 & 3 & 2.9 \\
\hline Jordan & 59 & 62.7 & -3 & -3.6 & 6 & 3.2 \\
\hline Montenegro & 60 & 62.5 & 7 & -1.3 & -3 & 2.0 \\
\hline Thailand & 61 & 62.2 & -4 & -3.5 & -3 & 1.8 \\
\hline Venezuela, RB & 62 & 61.8 & -4 & -3.2 & -3 & 1.9 \\
\hline Malaysia & 63 & 61.7 & -5 & -3.1 & 1 & 2.9 \\
\hline Morocco & 64 & 61.6 & 4 & -1.8 & 5 & 3.5 \\
\hline Azerbaijan & 65 & 61.3 & -2 & -2.7 & -3 & 2.1 \\
\hline Egypt, Arab Rep. & 66 & 60.9 & -9 & -4.3 & 5 & 3.7 \\
\hline Kyrgyz Republic & 67 & 60.9 & -2 & -2.4 & -5 & 1.9 \\
\hline Albania & 68 & 60.8 & 5 & -1.8 & 2 & 2.8 \\
\hline Mauritius & 69 & 60.7 & 5 & -2.0 & 0 & 2.4 \\
\hline Panama & 70 & 60.7 & -1 & -3.3 & -4 & 1.9 \\
\hline Ecuador & 71 & 60.7 & 1 & -3.0 & 0 & 2.0 \\
\hline Tajikistan & 72 & 60.2 & -1 & -3.4 & -4 & 1.8 \\
\hline Bosnia and Herzegovina & 73 & 59.9 & 12 & -0.4 & 3 & 3.1 \\
\hline Oman & 74 & 59.9 & 2 & -2.8 & 7 & 3.6 \\
\hline Paraguay & 75 & 59.3 & -4 & -3.8 & -2 & 2.1 \\
\hline China & 76 & 59.1 & 0 & -2.7 & -7 & 1.3 \\
\hline Jamaica & 77 & 59.1 & -3 & -3.5 & -2 & 1.7 \\
\hline Trinidad and Tobago & 78 & 59.1 & 4 & -2.4 & 0 & 1.8 \\
\hline Iran, Islamic Rep. & 79 & 58.5 & -8 & -4.0 & -2 & 1.9 \\
\hline Botswana & 80 & 58.4 & -2 & -3.2 & -5 & 1.8 \\
\hline Peru & 81 & 58.4 & 0 & -2.9 & -1 & 2.1 \\
\hline Bhutan & 82 & 58.2 & 5 & -2.0 & 2 & 2.4 \\
\hline Algeria & 83 & 58.1 & -1 & -3.4 & 10 & 4.5 \\
\hline Mongolia & 84 & 58.1 & -2 & -3.5 & -2 & 2.1 \\
\hline Saudi Arabia & 85 & 58.0 & 7 & -2.1 & 10 & 4.2 \\
\hline Lebanon & 86 & 58.0 & 1 & -3.3 & -2 & 1.7 \\
\hline Suriname & 87 & 58.0 & 4 & -2.9 & 0 & 1.8 \\
\hline Vietnam & 88 & 57.6 & -1 & -3.6 & -1 & 1.9 \\
\hline Bolivia & 89 & 57.5 & 1 & -3.1 & 5 & 2.8 \\
\hline Nicaragua & 90 & 57.4 & -1 & -3.7 & -1 & 1.7 \\
\hline Colombia & 91 & 57.2 & -1 & -3.7 & -1 & 1.7 \\
\hline Dominican Republic & 92 & 57.1 & -1 & -4.2 & 2 & 2.2 \\
\hline Gabon & 93 & 56.2 & 3 & -2.4 & 0 & 2.4 \\
\hline El Salvador & 94 & 55.6 & 0 & -2.9 & 0 & 2.5 \\
\hline Philippines & 95 & 55.5 & 0 & -2.8 & 0 & 2.5 \\
\hline Cabo Verde & 96 & 55.5 & -1 & -3.5 & -2 & 1.5 \\
\hline Sri Lanka & 97 & 54.8 & 1 & -2.3 & 1 & 2.4 \\
\hline Indonesia & 98 & 54.4 & -1 & -4.3 & -1 & 1.7 \\
\hline South Africa & 99 & 53.8 & -2 & -4.4 & 2 & 3.4 \\
\hline Kuwait & 100 & 52.5 & 2 & -1.5 & 0 & 2.9 \\
\hline Guyana & 101 & 52.4 & -1 & -3.5 & -2 & 2.0 \\
\hline Honduras & 102 & 51.8 & -2 & -3.5 & -2 & 2.5 \\
\hline Nepal & 103 & 51.5 & 3 & -1.9 & 2 & 3.2 \\
\hline Ghana & 104 & 51.4 & 1 & -2.8 & 2 & 3.1 \\
\hline Iraq & 105 & 50.9 & 0 & -3.4 & 0 & 3.0 \\
\hline Guatemala & 106 & 50.0 & -1 & -3.4 & 0 & 3.5 \\
\hline Lao PDR & 107 & 49.9 & 1 & -3.2 & -2 & 2.7 \\
\hline Namibia & 108 & 49.9 & -1 & -3.6 & 0 & 2.8 \\
\hline Zimbabwe & 109 & 48.6 & -2 & -4.3 & -1 & 3.0 \\
\hline India & 110 & 48.4 & 2 & -1.9 & 3 & 4.4 \\
\hline Congo, Rep. & 111 & 47.2 & 1 & -2.4 & -1 & 3.6 \\
\hline Cameroon & 112 & 46.3 & 0 & -2.6 & -2 & 3.2 \\
\hline Lesotho & 113 & 45.9 & -3 & -4.3 & -2 & 2.5 \\
\hline Senegal & 114 & 45.8 & 1 & -2.2 & 1 & 4.7 \\
\hline Pakistan & 115 & 45.7 & 1 & -3.3 & 4 & 5.4 \\
\hline Swaziland & 116 & 45.1 & -11 & -6.2 & -3 & 2.4 \\
\hline
\end{tabular}


On metrics and financing for the Sustainable Development Goals

\begin{tabular}{|c|c|c|c|c|c|c|}
\hline \multirow[t]{2}{*}{ Country } & \multicolumn{2}{|c|}{$\begin{array}{l}\text { SDG Index: Worst }(=0) \\
\text { - data truncated at } 2.5 \text { th } \\
\text { percentile }\end{array}$} & \multicolumn{2}{|c|}{$\begin{array}{c}\text { Worst }(=0) \\
\text { - data truncated at } 5 \text { th } \\
\text { percentile }\end{array}$} & \multicolumn{2}{|c|}{$\begin{array}{l}\text { Worst }(=0) \\
\text { - average of } 5 \text { worst per- } \\
\text { formers }\end{array}$} \\
\hline & Rank & Score & Rank diff. & Score diff. & Rank diff. & Score diff. \\
\hline Myanmar & 117 & 44.5 & -6 & -4.7 & -10 & 1.3 \\
\hline Bangladesh & 118 & 44.4 & 3 & -2.5 & 1 & 3.5 \\
\hline Cambodia & 119 & 44.4 & 2 & -2.9 & 3 & 3.6 \\
\hline Kenya & 120 & 44.0 & -5 & -4.8 & -3 & 2.6 \\
\hline Angola & 121 & 44.0 & 2 & -3.7 & -3 & 2.6 \\
\hline Rwanda & 122 & 44.0 & 1 & -3.8 & 0 & 3.0 \\
\hline Uganda & 123 & 43.6 & 3 & -3.3 & 2 & 3.5 \\
\hline Cote d'Ivoire & 124 & 43.5 & 6 & -3.1 & 6 & 4.2 \\
\hline Ethiopia & 125 & 43.1 & -3 & -4.2 & 5 & 4.2 \\
\hline Tanzania & 126 & 43.0 & 2 & -3.7 & 1 & 3.5 \\
\hline Sudan & 127 & 42.2 & 1 & -3.3 & 1 & 4.0 \\
\hline Burundi & 128 & 42.0 & 6 & -2.1 & -1 & 2.8 \\
\hline Togo & 129 & 40.9 & -1 & -4.0 & 1 & 4.4 \\
\hline Benin & 130 & 40.0 & -4 & -4.0 & 0 & 4.9 \\
\hline Malawi & 131 & 39.8 & -1 & -3.2 & -4 & 3.0 \\
\hline Mauritania & 132 & 39.6 & 3 & -1.4 & 1 & 4.7 \\
\hline Mozambique & 133 & 39.5 & 2 & -2.9 & 1 & 4.5 \\
\hline Zambia & 134 & 38.4 & -3 & -4.1 & -3 & 3.2 \\
\hline Mali & 135 & 38.2 & 2 & -1.8 & 2 & 5.3 \\
\hline Gambia, The & 136 & 37.8 & 1 & -2.7 & 2 & 5.3 \\
\hline Yemen, Rep. & 137 & 37.3 & -4 & -5.2 & 1 & 4.6 \\
\hline Sierra Leone & 138 & 36.9 & 2 & -2.5 & 0 & 4.5 \\
\hline Afghanistan & 139 & 36.5 & -3 & -4.5 & -4 & 2.3 \\
\hline Madagascar & 140 & 36.2 & -3 & -4.3 & -2 & 2.9 \\
\hline Nigeria & 141 & 36.1 & 2 & -3.0 & 1 & 4.2 \\
\hline Guinea & 142 & 35.9 & 2 & -3.0 & 3 & 5.2 \\
\hline Burkina Faso & 143 & 35.6 & 5 & -2.0 & 2 & 4.3 \\
\hline Haiti & 144 & 34.4 & 0 & -3.8 & 0 & 4.1 \\
\hline Chad & 145 & 31.8 & -2 & -3.5 & 0 & 5.7 \\
\hline Niger & 146 & 31.4 & 0 & -2.7 & 0 & 4.6 \\
\hline Congo, Dem. Rep. & 147 & 31.3 & 2 & -2.3 & 0 & 4.5 \\
\hline Liberia & 148 & 30.5 & 0 & -3.6 & 0 & 4.6 \\
\hline Central African Republic & 149 & 26.1 & 0 & -1.5 & 0 & 4.5 \\
\hline
\end{tabular}


Table 14 | SDG Indices obtained by arithmetic and geometric average

Comparison of SDG Index rank and score for each country using the arithmetic mean and the geometric mean across SDG scores. The median rank difference records the difference between the rank based on the arithmetic mean and the median rank.

\begin{tabular}{|c|c|c|c|c|c|}
\hline \multirow[t]{2}{*}{ Country } & \multicolumn{2}{|c|}{$\begin{array}{l}\text { Arithmetic } \\
\text { mean }\end{array}$} & \multicolumn{2}{|c|}{$\begin{array}{c}\text { Geometric } \\
\text { mean }\end{array}$} & \multirow{2}{*}{$\begin{array}{c}\text { Median } \\
\text { rank } \\
\text { difference }\end{array}$} \\
\hline & Rank & Score & Rank & Score & \\
\hline Sweden & 1 & 84.5 & 1 & 83.7 & 0 \\
\hline Denmark & 2 & 83.9 & 2 & 83.1 & 0 \\
\hline Norway & 3 & 82.3 & 3 & 81.1 & 0 \\
\hline Finland & 4 & 81.0 & 5 & 79.8 & -1 \\
\hline Switzerland & 5 & 80.9 & 4 & 80.2 & 1 \\
\hline Germany & 6 & 80.5 & 6 & 79.6 & 0 \\
\hline Austria & 7 & 79.1 & 7 & 77.9 & 0 \\
\hline Netherlands & 8 & 78.9 & 8 & 77.5 & 0 \\
\hline Iceland & 9 & 78.4 & 15 & 75.2 & -3 \\
\hline $\begin{array}{l}\text { United } \\
\text { Kingdom }\end{array}$ & 10 & 78.1 & 10 & 76.7 & 0 \\
\hline France & 11 & 77.9 & 9 & 76.7 & 1 \\
\hline Belgium & 12 & 77.4 & 11 & 76.1 & 1 \\
\hline Canada & 13 & 76.8 & 14 & 75.3 & -1 \\
\hline Ireland & 14 & 76.7 & 12 & 75.4 & 1 \\
\hline $\begin{array}{l}\text { Czech } \\
\text { Republic }\end{array}$ & 15 & 76.7 & 17 & 73.6 & -1 \\
\hline Luxembourg & 16 & 76.7 & 13 & 75.4 & 2 \\
\hline Slovenia & 17 & 76.6 & 16 & 74.8 & 1 \\
\hline Japan & 18 & 75.0 & 19 & 72.8 & -1 \\
\hline Singapore & 19 & 74.6 & 28 & 70.3 & -5 \\
\hline Australia & 20 & 74.5 & 20 & 71.9 & 0 \\
\hline Estonia & 21 & 74.5 & 18 & 73.1 & 2 \\
\hline New Zealand & 22 & 74.0 & 22 & 71.2 & 0 \\
\hline Belarus & 23 & 73.5 & 24 & 70.5 & -1 \\
\hline Hungary & 24 & 73.4 & 21 & 71.5 & 2 \\
\hline United States & 25 & 72.7 & 23 & 70.5 & 1 \\
\hline $\begin{array}{l}\text { Slovak } \\
\text { Republic }\end{array}$ & 26 & 72.7 & 31 & 69.0 & -3 \\
\hline South Korea & 27 & 72.7 & 30 & 69.1 & -2 \\
\hline Latvia & 28 & 72.5 & 26 & 70.4 & 1 \\
\hline Israel & 29 & 72.3 & 25 & 70.5 & 2 \\
\hline Spain & 30 & 72.2 & 33 & 68.9 & -2 \\
\hline Lithuania & 31 & 72.1 & 29 & 70.2 & 1 \\
\hline Malta & 32 & 72.0 & 27 & 70.4 & 3 \\
\hline Bulgaria & 33 & 71.8 & 32 & 69.0 & 1 \\
\hline Portugal & 34 & 71.5 & 36 & 68.2 & -1 \\
\hline Italy & 35 & 70.9 & 35 & 68.3 & 0 \\
\hline Croatia & 36 & 70.7 & 34 & 68.5 & 1 \\
\hline Greece & 37 & 69.9 & 37 & 66.4 & 0 \\
\hline Poland & 38 & 69.8 & 38 & 65.9 & 0 \\
\hline Serbia & 39 & 68.3 & 39 & 65.1 & 0 \\
\hline Uruguay & 40 & 68.0 & 40 & 64.2 & 0 \\
\hline Romania & 41 & 67.5 & 42 & 63.7 & -1 \\
\hline Chile & 42 & 67.2 & 44 & 63.5 & -1 \\
\hline Argentina & 43 & 66.8 & 47 & 62.9 & -2 \\
\hline Moldova & 44 & 66.6 & 43 & 63.5 & 1 \\
\hline Cyprus & 45 & 66.5 & 48 & 62.6 & -2 \\
\hline Ukraine & 46 & 66.4 & 51 & 60.3 & -3 \\
\hline $\begin{array}{l}\text { Russian } \\
\text { Federation }\end{array}$ & 47 & 66.4 & 41 & 63.8 & 3 \\
\hline Turkey & 48 & 66.1 & 46 & 63.0 & 1 \\
\hline Qatar & 49 & 65.8 & 45 & 63.1 & 2 \\
\hline Armenia & 50 & 65.4 & 53 & 59.9 & -2 \\
\hline Tunisia & 51 & 65.1 & 49 & 62.0 & 1 \\
\hline Brazil & 52 & 64.4 & 50 & 60.4 & 1 \\
\hline Costa Rica & 53 & 64.2 & 52 & 60.0 & 1 \\
\hline Kazakhstan & 54 & 63.9 & 59 & 58.6 & -3 \\
\hline $\begin{array}{l}\text { United Arab } \\
\text { Emirates }\end{array}$ & 55 & 63.6 & 58 & 59.2 & -2 \\
\hline Mexico & 56 & 63.4 & 57 & 59.2 & -1 \\
\hline Georgia & 57 & 63.3 & 54 & 59.6 & 2 \\
\hline $\begin{array}{l}\text { Macedonia, } \\
\text { FYR }\end{array}$ & 58 & 62.8 & 60 & 58.5 & -1 \\
\hline Jordan & 59 & 62.7 & 61 & 57.2 & -1 \\
\hline Montenegro & 60 & 62.5 & 80 & 53.5 & -10 \\
\hline
\end{tabular}

\begin{tabular}{|c|c|c|c|c|c|}
\hline \multirow[t]{2}{*}{ Country } & \multicolumn{2}{|c|}{$\begin{array}{c}\text { Arithmetic } \\
\text { mean }\end{array}$} & \multicolumn{2}{|c|}{$\begin{array}{c}\text { Geometric } \\
\text { mean }\end{array}$} & \multirow{2}{*}{$\begin{array}{c}\text { Median } \\
\text { rank } \\
\text { difference }\end{array}$} \\
\hline & Rank & Score & Rank & Score & \\
\hline Thailand & 61 & 62.2 & 55 & 59.4 & 3 \\
\hline $\begin{array}{l}\text { Venezuela, } \\
\text { RB }\end{array}$ & 62 & 61.8 & 65 & 56.6 & -2 \\
\hline Malaysia & 63 & 61.7 & 63 & 56.7 & 0 \\
\hline Morocco & 64 & 61.6 & 56 & 59.3 & 4 \\
\hline Azerbaijan & 65 & 61.3 & 68 & 55.9 & -2 \\
\hline $\begin{array}{l}\text { Egypt, Arab } \\
\text { Rep. }\end{array}$ & 66 & 60.9 & 66 & 56.3 & 0 \\
\hline $\begin{array}{l}\text { Kyrgyz } \\
\text { Republic }\end{array}$ & 67 & 60.9 & 88 & 50.6 & -11 \\
\hline Albania & 68 & 60.8 & 62 & 56.7 & 3 \\
\hline Mauritius & 69 & 60.7 & 75 & 54.2 & -3 \\
\hline Panama & 70 & 60.7 & 67 & 55.9 & 2 \\
\hline Ecuador & 71 & 60.7 & 64 & 56.6 & 4 \\
\hline Tajikistan & 72 & 60.2 & 71 & 54.7 & 1 \\
\hline $\begin{array}{l}\text { Bosnia and } \\
\text { Herzegovina }\end{array}$ & 73 & 59.9 & 92 & 49.7 & -10 \\
\hline Oman & 74 & 59.9 & 76 & 54.0 & -1 \\
\hline Paraguay & 75 & 59.3 & 78 & 53.6 & -2 \\
\hline China & 76 & 59.1 & 69 & 55.8 & 4 \\
\hline Jamaica & 77 & 59.1 & 72 & 54.7 & 3 \\
\hline $\begin{array}{l}\text { Trinidad and } \\
\text { Tobago }\end{array}$ & 78 & 59.1 & 97 & 48.5 & -10 \\
\hline $\begin{array}{l}\text { Iran, Islamic } \\
\text { Rep. }\end{array}$ & 79 & 58.5 & 77 & 53.9 & 1 \\
\hline Botswana & 80 & 58.4 & 70 & 55.2 & 5 \\
\hline Peru & 81 & 58.4 & 81 & 53.3 & 0 \\
\hline Bhutan & 82 & 58.2 & 74 & 54.2 & 4 \\
\hline Algeria & 83 & 58.1 & 79 & 53.6 & 2 \\
\hline Mongolia & 84 & 58.1 & 73 & 54.2 & 6 \\
\hline Saudi Arabia & 85 & 58.0 & 113 & 39.0 & -14 \\
\hline Lebanon & 86 & 58.0 & 84 & 52.9 & 1 \\
\hline Suriname & 87 & 58.0 & 82 & 53.2 & 3 \\
\hline Vietnam & 88 & 57.6 & 83 & 52.9 & 3 \\
\hline Bolivia & 89 & 57.5 & 85 & 52.5 & 2 \\
\hline Nicaragua & 90 & 57.4 & 87 & 51.3 & 2 \\
\hline Colombia & 91 & 57.2 & 89 & 50.6 & 1 \\
\hline $\begin{array}{l}\text { Dominican } \\
\text { Republic }\end{array}$ & 92 & 57.1 & 93 & 49.3 & -1 \\
\hline Gabon & 93 & 56.2 & 90 & 50.4 & 2 \\
\hline El Salvador & 94 & 55.6 & 95 & 49.1 & -1 \\
\hline Philippines & 95 & 55.5 & 91 & 50.4 & 2 \\
\hline Cabo Verde & 96 & 55.5 & 86 & 51.6 & 5 \\
\hline Sri Lanka & 97 & 54.8 & 116 & 38.2 & -10 \\
\hline Indonesia & 98 & 54.4 & 96 & 48.8 & 1 \\
\hline South Africa & 99 & 53.8 & 118 & 37.4 & -10 \\
\hline Kuwait & 100 & 52.5 & 129 & 32.9 & -15 \\
\hline Guyana & 101 & 52.4 & 112 & 39.2 & -6 \\
\hline Honduras & 102 & 51.8 & 100 & 45.2 & 1 \\
\hline Nepal & 103 & 51.5 & 99 & 45.9 & 2 \\
\hline Ghana & 104 & 51.4 & 94 & 49.2 & 5 \\
\hline Iraq & 105 & 50.9 & 106 & 42.7 & -1 \\
\hline Guatemala & 106 & 50.0 & 103 & 43.7 & 2 \\
\hline Lao PDR & 107 & 49.9 & 98 & 46.0 & 5 \\
\hline Namibia & 108 & 49.9 & 125 & 34.6 & -9 \\
\hline Zimbabwe & 109 & 48.6 & 101 & 44.4 & 4 \\
\hline India & 110 & 48.4 & 102 & 44.3 & 4 \\
\hline Congo, Rep. & 111 & 47.2 & 127 & 33.4 & -8 \\
\hline Cameroon & 112 & 46.3 & 109 & 39.7 & 2 \\
\hline Lesotho & 113 & 45.9 & 110 & 39.6 & 2 \\
\hline Senegal & 114 & 45.8 & 104 & 42.9 & 5 \\
\hline Pakistan & 115 & 45.7 & 120 & 36.3 & -3 \\
\hline Swaziland & 116 & 45.1 & 107 & 42.2 & 5 \\
\hline Myanmar & 117 & 44.5 & 121 & 35.3 & -2 \\
\hline Bangladesh & 118 & 44.4 & 124 & 34.7 & -3 \\
\hline Cambodia & 119 & 44.4 & 132 & 28.8 & -7 \\
\hline
\end{tabular}


On metrics and financing for the Sustainable Development Goals

\begin{tabular}{|c|c|c|c|c|c|}
\hline \multirow[t]{2}{*}{ Country } & \multicolumn{2}{|c|}{$\begin{array}{l}\text { Arithmetic } \\
\text { mean }\end{array}$} & \multicolumn{2}{|c|}{$\begin{array}{c}\text { Geometric } \\
\text { mean }\end{array}$} & \multirow{2}{*}{$\begin{array}{l}\text { Median } \\
\text { rank } \\
\text { difference }\end{array}$} \\
\hline & Rank & Score & Rank & Score & \\
\hline Kenya & 120 & 44.0 & 105 & 42.7 & 8 \\
\hline Angola & 121 & 44.0 & 108 & 40.5 & 7 \\
\hline Rwanda & 122 & 44.0 & 119 & 36.6 & 2 \\
\hline Uganda & 123 & 43.6 & 117 & 37.6 & 3 \\
\hline Cote d'Ivoire & 124 & 43.5 & 114 & 38.5 & 5 \\
\hline Ethiopia & 125 & 43.1 & 115 & 38.5 & 5 \\
\hline Tanzania & 126 & 43.0 & 111 & 39.2 & 8 \\
\hline Sudan & 127 & 42.2 & 135 & 28.2 & -4 \\
\hline Burundi & 128 & 42.0 & 143 & 19.6 & -8 \\
\hline Togo & 129 & 40.9 & 123 & 34.7 & 3 \\
\hline Benin & 130 & 40.0 & 122 & 34.8 & 4 \\
\hline Malawi & 131 & 39.8 & 144 & 19.6 & -7 \\
\hline Mauritania & 132 & 39.6 & 126 & 34.4 & 3 \\
\hline Mozambique & 133 & 39.5 & 136 & 26.4 & -2 \\
\hline Zambia & 134 & 38.4 & 130 & 31.5 & 2 \\
\hline Mali & 135 & 38.2 & 131 & 30.9 & 2 \\
\hline Gambia, The & 136 & 37.8 & 128 & 33.4 & 4 \\
\hline
\end{tabular}

\begin{tabular}{|l|r|r|r|r|r|}
\multirow{2}{*}{ Country } & \multicolumn{2}{c|}{$\begin{array}{c}\text { Arithmetic } \\
\text { mean }\end{array}$} & \multicolumn{2}{c|}{$\begin{array}{c}\text { Geometric } \\
\text { mean }\end{array}$} & $\begin{array}{c}\text { Median } \\
\text { rank } \\
\text { difference }\end{array}$ \\
\cline { 2 - 5 } & Rank & Score & Rank & Score & -1 \\
\hline Yemen, Rep. & 137 & 37.3 & 138 & 24.6 & 3 \\
\hline Sierra Leone & 138 & 36.9 & 133 & 28.4 & -2 \\
\hline Afghanistan & 139 & 36.5 & 142 & 20.2 & -1 \\
\hline Madagascar & 140 & 36.2 & 141 & 20.6 & -4 \\
\hline Nigeria & 141 & 36.1 & 148 & 10.7 & 2 \\
\hline Guinea & 142 & 35.9 & 139 & 22.4 & 3 \\
\hline Burkina Faso & 143 & 35.6 & 137 & 25.5 & 5 \\
\hline Haiti & 144 & 34.4 & 134 & 28.3 & 3 \\
\hline Chad & 145 & 31.8 & 140 & 22.2 & 1 \\
\hline Niger & 146 & 31.4 & 146 & 17.0 & 1 \\
\hline Congo, Dem. & 147 & 31.3 & 145 & 17.5 & 0 \\
Rep. & 148 & 30.5 & 147 & 12.0 & \\
\hline Liberia & 149 & 26.1 & 149 & 3.4 & \\
\hline Central & & & & & \\
African & & & & & \\
Republic & & & & & \\
\hline
\end{tabular}


Table 15 | Country scores by SDG.

Overall SDG Index score and average country scores by each SDG. n.d. - no data.

\begin{tabular}{|c|c|c|c|c|c|c|c|c|c|c|c|c|c|c|c|c|c|c|}
\hline Country & $\begin{array}{l}\text { SDG } \\
\text { Index }\end{array}$ & $\begin{array}{c}\text { SDG } \\
1\end{array}$ & $\begin{array}{l}\text { SDG } \\
2\end{array}$ & $\begin{array}{l}\text { SDG } \\
3\end{array}$ & $\begin{array}{l}\text { SDG } \\
4\end{array}$ & $\begin{array}{c}\text { SDG } \\
5\end{array}$ & $\begin{array}{l}\text { SDG } \\
6\end{array}$ & $\begin{array}{c}\text { SDG } \\
7\end{array}$ & $\begin{array}{l}\text { SDG } \\
8\end{array}$ & $\begin{array}{l}\text { SDG } \\
9\end{array}$ & $\begin{array}{c}\text { SDG } \\
10\end{array}$ & $\begin{array}{c}\text { SDG } \\
11\end{array}$ & $\begin{array}{c}\text { SDG } \\
12\end{array}$ & $\begin{array}{c}\text { SDG } \\
13\end{array}$ & $\begin{array}{c}\text { SDG } \\
14\end{array}$ & $\begin{array}{c}\text { SDG } \\
15\end{array}$ & $\begin{array}{c}\text { SDG } \\
16\end{array}$ & $\begin{array}{c}\text { SDG } \\
17\end{array}$ \\
\hline Afghanistan & 36.5 & n.d. & 30.1 & 34.3 & 14.9 & 28.7 & 41.1 & 23.6 & 44.3 & 1.6 & 93.5 & 41.0 & - & 83.2 & n.d. & 52.7 & 40.4 & 18.1 \\
\hline Albania & 60.8 & 98.5 & 43.1 & 72.1 & 69.5 & 49.9 & 93.7 & 81.9 & 49.5 & 28.9 & 75.8 & 76.2 & 45.7 & 63.3 & 53.3 & 60.4 & 51.1 & 20.3 \\
\hline Algeria & 58.1 & n.d. & 56.9 & 63.4 & 78.1 & 41.3 & 78.2 & 81.3 & 51.0 & 12.5 & n.d. & 70.7 & 57.2 & 85.5 & 50.4 & 59.9 & 52.1 & 33.5 \\
\hline Angola & 44.0 & 56.2 & 37.5 & 16.9 & 45.2 & 42.7 & 48.3 & 29.9 & 42.3 & 13.6 & 54.2 & 49.1 & 46.8 & 86.2 & 32.1 & 66.7 & 40.6 & 40.0 \\
\hline Argentina & 66.8 & 100.0 & 76.1 & 76.2 & 91.4 & 74.8 & 97.6 & 86.5 & 63.0 & 31.7 & 49.4 & 89.7 & 45.7 & 82.5 & 39.0 & 45.4 & 42.7 & 44.3 \\
\hline Armenia & 65.4 & 96.5 & 56.2 & 69.8 & 63.9 & 54.9 & 92.6 & 87.5 & 57.0 & 28.5 & 84.3 & 81.5 & 56.2 & 89.4 & n.d. & 55.0 & 59.4 & 13.9 \\
\hline Australia & 74.5 & 100.0 & 69.4 & 86.3 & 95.8 & 77.6 & 99.7 & 84.3 & 79.3 & 74.3 & 80.8 & 87.7 & 76.5 & 40.7 & 50.0 & 50.7 & 72.3 & 41.4 \\
\hline Austria & 79.1 & 100.0 & 81.2 & 86.4 & 82.9 & 72.9 & 99.6 & 89.3 & 73.9 & 71.8 & 90.0 & 84.6 & 68.2 & 78.8 & n.d. & 55.1 & 77.1 & 53.3 \\
\hline Azerbaijan & 61.3 & 100.0 & 58.7 & 69.2 & 68.2 & 46.5 & 84.1 & 86.4 & 59.7 & 38.1 & 77.9 & 72.9 & 13.1 & 70.2 & n.d. & 59.3 & 49.1 & 27.9 \\
\hline Bangladesh & 44.4 & 36.5 & 38.3 & 50.8 & 54.3 & 51.4 & 75.9 & 43.2 & 55.0 & 5.8 & 82.1 & 14.0 & 47.3 & 64.9 & 42.9 & 44.7 & 45.8 & 2.4 \\
\hline Belarus & 73.5 & 100.0 & 60.2 & 78.4 & 85.3 & 72.1 & 97.4 & 81.8 & 62.7 & 33.4 & 97.0 & 84.7 & 87.2 & 81.4 & n.d. & 58.9 & 55.6 & 39.9 \\
\hline Belgium & 77.4 & 100.0 & 98.6 & 86.5 & 85.5 & 82.7 & 96.8 & 88.6 & 67.0 & 65.8 & 79.9 & 80.8 & 68.8 & 70.9 & 50.1 & 63.3 & 71.5 & 59.5 \\
\hline Benin & 40.0 & 22.7 & 45.8 & 29.1 & 40.3 & 31.4 & 54.5 & 17.9 & 48.3 & 5.5 & 64.9 & 37.4 & 46.2 & 72.5 & 34.7 & 60.9 & 47.9 & 19.7 \\
\hline Bhutan & 58.2 & 96.8 & 40.3 & 59.7 & 55.0 & 46.8 & 81.2 & 65.6 & 67.6 & 23.7 & 66.4 & 60.2 & 37.6 & 90.3 & n.d. & 50.3 & 65.1 & 24.3 \\
\hline Bolivia & 57.5 & 88.8 & 57.2 & 52.2 & 63.1 & 70.8 & 74.3 & 67.3 & 48.7 & 17.4 & 18.1 & 86.7 & 53.8 & 81.7 & n.d. & 58.0 & 41.3 & 40.2 \\
\hline $\begin{array}{l}\text { Bosnia and } \\
\text { Herzegovina }\end{array}$ & 59.9 & 99.9 & 59.9 & 72.8 & 82.6 & 40.0 & 97.9 & 73.2 & 37.7 & 24.6 & 71.3 & 82.2 & 3.2 & 79.9 & 30.6 & 61.3 & 54.6 & 46.6 \\
\hline Botswana & 58.4 & 73.5 & 35.1 & 47.7 & 68.9 & 63.9 & 83.4 & 36.3 & 49.2 & 29.8 & n.d. & 88.1 & 42.1 & 87.9 & n.d. & 75.0 & 48.2 & 47.1 \\
\hline Brazil & 64.4 & 92.9 & 73.9 & 68.6 & 74.6 & 67.6 & 92.1 & 90.8 & 64.3 & 37.3 & 22.4 & 81.7 & 47.0 & 86.9 & 54.1 & 57.7 & 34.5 & 49.0 \\
\hline Bulgaria & 71.8 & 97.0 & 64.0 & 74.9 & 80.2 & 66.7 & 91.7 & 89.4 & 57.5 & 38.7 & 92.5 & 83.1 & 53.7 & 81.3 & 74.1 & 88.6 & 51.7 & 35.4 \\
\hline Burkina Faso & 35.6 & 19.6 & 32.0 & 34.4 & 2.2 & 21.5 & 57.0 & 3.5 & 30.7 & 9.7 & 61.8 & 30.7 & 46.5 & 82.6 & n.d. & 53.0 & 62.5 & 22.5 \\
\hline Burundi & 42.0 & - & 25.9 & 33.5 & 63.0 & 51.4 & 63.8 & - & 32.7 & 5.9 & 79.1 & 55.4 & 46.1 & 69.7 & n.d. & 69.8 & 39.1 & 37.4 \\
\hline Cabo Verde & 55.5 & 74.4 & 38.7 & 62.4 & 81.0 & 60.4 & 83.2 & 67.0 & 50.3 & 27.6 & 33.4 & 35.7 & 56.3 & 95.9 & 48.3 & 54.1 & 53.0 & 21.1 \\
\hline Cambodia & 44.4 & 91.1 & 44.7 & 52.5 & 68.2 & 51.1 & 61.5 & 17.4 & 55.4 & 12.4 & 71.8 & 66.4 & - & 49.3 & 20.2 & 33.3 & 39.9 & 18.9 \\
\hline Cameroon & 46.3 & 57.4 & 44.1 & 29.2 & 59.4 & 57.4 & 62.9 & 48.7 & 31.7 & 6.3 & 64.1 & 39.4 & 44.1 & 91.5 & 40.7 & 57.5 & 43.8 & 9.4 \\
\hline Canada & 76.8 & 100.0 & 78.7 & 84.4 & 84.9 & 80.1 & 99.7 & 90.9 & 85.2 & 63.8 & 81.0 & 87.5 & 69.6 & 62.2 & 55.9 & 57.7 & 79.2 & 45.8 \\
\hline $\begin{array}{l}\text { Central African } \\
\text { Republic }\end{array}$ & 26.1 & 3.6 & 23.1 & 4.3 & - & 16.7 & 49.0 & 0.6 & 26.0 & 7.8 & 18.1 & 30.0 & 46.6 & 55.1 & n.d. & 81.7 & 55.1 & 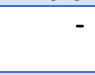 \\
\hline Chad & 31.8 & 44.1 & 15.4 & 10.7 & 24.1 & 9.9 & 33.2 & 3.6 & 37.1 & 3.6 & 61.8 & 28.2 & 46.6 & 78.8 & n.d. & 70.9 & 32.1 & 8.5 \\
\hline Chile & 67.2 & 100.0 & 75.2 & 75.0 & 80.0 & 65.0 & 98.6 & 87.6 & 64.1 & 41.7 & 29.3 & 80.8 & 75.6 & 83.7 & 43.3 & 39.3 & 60.4 & 42.4 \\
\hline China & 59.1 & 83.7 & 67.8 & 73.2 & 81.0 & 71.0 & 86.3 & 76.0 & 70.0 & 45.5 & 55.8 & 43.2 & 50.8 & 39.2 & 32.0 & 48.7 & 56.7 & 23.3 \\
\hline Colombia & 57.2 & 91.1 & 57.2 & 68.4 & 70.3 & 70.5 & 87.0 & 84.5 & 56.8 & 23.6 & 19.1 & 83.8 & 44.7 & 88.4 & 17.9 & 41.1 & 37.5 & 30.7 \\
\hline Congo, Dem. Rep. & 31.3 & & 23.4 & 19.5 & 32.4 & 15.6 & 40.7 & 33.8 & 32.2 & 1.6 & 49.5 & 37.1 & 46.6 & 90.7 & 15.4 & 56.7 & 33.2 & 3.5 \\
\hline Congo, Rep. & 47.2 & 58.2 & 34.0 & 32.5 & 50.6 & 38.1 & 51.8 & 40.0 & 42.8 & 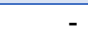 & 41.9 & 52.0 & 46.4 & 92.1 & 35.2 & 79.2 & 58.5 & 48.8 \\
\hline Costa Rica & 64.2 & 97.6 & 62.0 & 74.0 & 74.1 & 76.9 & 94.6 & 91.7 & 63.8 & 34.8 & 32.9 & 90.1 & 38.9 & 88.0 & 40.1 & 46.5 & 50.4 & 35.6 \\
\hline Cote d'Ivoire & 43.5 & 57.8 & 43.1 & 21.9 & 18.4 & 23.4 & 58.2 & 46.0 & 37.9 & 19.2 & 57.3 & 60.8 & 47.1 & 91.4 & 45.7 & 48.7 & 50.6 & 11.9 \\
\hline Croatia & 70.7 & 100.0 & 68.4 & 84.7 & 76.4 & 58.4 & 98.5 & 86.6 & 61.9 & 47.2 & 78.1 & 85.4 & 55.2 & 84.6 & 62.0 & 64.7 & 50.1 & 39.9 \\
\hline Cyprus & 66.5 & 100.0 & 33.0 & 82.5 & 83.2 & 66.4 & 98.4 & 86.6 & 50.9 & 39.0 & n.d. & 83.0 & 36.5 & 77.3 & 36.3 & 70.1 & 65.6 & 55.7 \\
\hline Czech Republic & 76.7 & 100.0 & 78.6 & 89.0 & 86.3 & 70.1 & 98.4 & 87.8 & 66.3 & 56.5 & 99.4 & 82.9 & 78.8 & 72.4 & n.d. & 73.3 & 62.9 & 25.1 \\
\hline Denmark & 83.9 & 100.0 & 77.3 & 86.8 & 95.3 & 84.3 & 98.9 & 88.3 & 65.1 & 83.0 & 100.0 & 88.2 & 76.0 & 72.1 & 59.7 & 80.6 & 80.4 & 89.9 \\
\hline
\end{tabular}




\begin{tabular}{|c|c|c|c|c|c|c|c|c|c|c|c|c|c|c|c|c|c|c|}
\hline Country & $\begin{array}{c}\text { SDG } \\
\text { Index }\end{array}$ & $\begin{array}{c}\text { SDG } \\
1\end{array}$ & $\begin{array}{c}\text { SDG } \\
2\end{array}$ & $\begin{array}{c}\text { SDG } \\
3\end{array}$ & $\begin{array}{c}\text { SDG } \\
4\end{array}$ & $\begin{array}{c}\text { SDG } \\
5\end{array}$ & $\begin{array}{c}\text { SDG } \\
6\end{array}$ & $\begin{array}{c}\text { SDG } \\
7\end{array}$ & $\begin{array}{c}\text { SDG } \\
8\end{array}$ & $\begin{array}{c}\text { SDG } \\
9\end{array}$ & $\begin{array}{c}\text { SDG } \\
10\end{array}$ & $\begin{array}{c}\text { SDG } \\
11\end{array}$ & $\begin{array}{c}\text { SDG } \\
12\end{array}$ & $\begin{array}{c}\text { SDG } \\
13\end{array}$ & $\begin{array}{c}\text { SDG } \\
14\end{array}$ & $\begin{array}{c}\text { SDG } \\
15\end{array}$ & $\begin{array}{c}\text { SDG } \\
16\end{array}$ & $\begin{array}{c}\text { SDG } \\
17\end{array}$ \\
\hline $\begin{array}{l}\text { Dominican } \\
\text { Republic }\end{array}$ & 57.1 & 96.6 & 57.4 & 54.6 & 67.3 & 69.7 & 80.9 & 86.9 & 51.3 & 30.5 & 42.2 & 74.2 & 43.1 & 88.2 & 41.2 & 46.2 & 35.7 & 4.8 \\
\hline Ecuador & 60.7 & 93.6 & 51.0 & 65.2 & 82.2 & 75.1 & 85.1 & 82.1 & 63.6 & 25.1 & 36.7 & 81.8 & 43.9 & 85.2 & 43.2 & 37.2 & 45.2 & 35.7 \\
\hline Egypt, Arab Rep. & 60.9 & n.d. & 62.4 & 67.9 & 73.2 & 35.6 & 86.3 & 88.5 & 44.6 & 24.5 & 85.7 & 62.4 & 63.2 & 81.9 & 48.6 & 69.0 & 60.9 & 19.7 \\
\hline El Salvador & 55.6 & 95.3 & 52.5 & 66.6 & 69.3 & 67.1 & 85.6 & 81.0 & 47.4 & 19.3 & 39.2 & 80.2 & 41.0 & 89.5 & 20.8 & 44.6 & 26.7 & 19.8 \\
\hline Estonia & 74.5 & 100.0 & 57.1 & 81.0 & 87.0 & 73.2 & 97.5 & 83.4 & 74.3 & 65.2 & 71.8 & 90.6 & 75.1 & 61.1 & 67.3 & 70.8 & 66.1 & 44.4 \\
\hline Ethiopia & 43.1 & 51.2 & 29.8 & 38.6 & 29.9 & 52.6 & 43.4 & 30.8 & 48.4 & 8.1 & 78.2 & 58.5 & 48.5 & 64.3 & n.d. & 52.3 & 37.5 & 17.0 \\
\hline Finland & 81.0 & 100.0 & 60.4 & 87.1 & 89.8 & 88.4 & 98.6 & 92.1 & 58.4 & 85.1 & 96.0 & 92.7 & 73.4 & 71.7 & 68.2 & 62.2 & 86.3 & 66.5 \\
\hline France & 77.9 & 100.0 & 74.7 & 84.8 & 84.4 & 78.8 & 98.1 & 93.3 & 67.6 & 69.5 & 86.1 & 85.5 & 75.1 & 83.6 & 55.2 & 60.4 & 71.3 & 55.9 \\
\hline Gabon & 56.2 & 88.4 & 51.4 & 32.5 & 66.7 & 52.6 & 73.4 & 76.7 & 41.2 & 9.4 & 57.4 & 73.7 & 47.1 & 87.4 & 47.4 & 79.4 & 44.0 & 27.1 \\
\hline Gambia, The & 37.8 & 34.1 & 40.6 & 37.0 & 20.8 & 35.2 & 77.7 & 15.8 & 17.4 & 11.4 & 42.0 & 38.7 & 46.5 & 71.8 & 32.4 & 51.3 & 50.2 & 19.2 \\
\hline Georgia & 63.3 & 83.3 & 53.9 & 72.8 & 81.2 & 57.2 & 94.6 & 78.1 & 56.1 & 25.6 & 55.7 & 81.9 & 35.4 & 87.2 & 69.3 & 53.7 & 60.5 & 29.3 \\
\hline Germany & 80.5 & 100.0 & 86.8 & 87.3 & 85.1 & 79.3 & 97.8 & 87.5 & 76.7 & 72.9 & 92.2 & 84.1 & 79.0 & 74.7 & 51.8 & 72.6 & 78.2 & 62.8 \\
\hline Ghana & 51.4 & 63.4 & 52.9 & 43.2 & 58.9 & 47.1 & 59.9 & 48.0 & 47.6 & 21.6 & 53.9 & 36.5 & 55.1 & 85.9 & 48.3 & 65.4 & 57.8 & 28.2 \\
\hline Greece & 69.9 & 100.0 & 64.2 & 85.2 & 95.2 & 61.2 & 98.4 & 87.5 & 40.4 & 40.3 & 76.4 & 84.1 & 76.1 & 75.3 & 56.4 & 61.9 & 56.3 & 29.2 \\
\hline Guatemala & 50.0 & 83.2 & 37.8 & 56.9 & 57.4 & 53.0 & 81.2 & 61.2 & 45.7 & 17.2 & 19.2 & 85.8 & 35.2 & 86.4 & 46.9 & 31.6 & 39.0 & 12.4 \\
\hline Guinea & 35.9 & 48.7 & 36.9 & 19.7 & 10.9 & 30.0 & 54.0 & 9.1 & 31.4 & 1.7 & 63.0 & 38.5 & 0.8 & 90.2 & 55.8 & 69.7 & 38.8 & 11.7 \\
\hline Guyana & 52.4 & n.d. & 64.7 & 54.3 & 41.1 & 71.3 & 92.6 & 84.9 & 47.2 & 19.9 & n.d. & 78.1 & 1.1 & 44.4 & 37.9 & 88.5 & 39.4 & 19.9 \\
\hline Haiti & 34.4 & 21.6 & 34.0 & 38.5 & 31.2 & 46.4 & 43.0 & 27.3 & 46.9 & 3.0 & 10.4 & 40.0 & 41.9 & 87.6 & 25.4 & 36.4 & 37.7 & 13.3 \\
\hline Honduras & 51.8 & 72.5 & 47.4 & 59.0 & 63.4 & 65.2 & 87.3 & 66.2 & 48.9 & 17.8 & 16.4 & 89.7 & 45.4 & 84.2 & 24.0 & 39.4 & 37.3 & 16.2 \\
\hline Hungary & 73.4 & 100.0 & 75.4 & 79.1 & 79.4 & 63.6 & 98.8 & 86.6 & 66.9 & 46.2 & 84.6 & 82.5 & 62.5 & 85.7 & n.d. & 60.9 & 51.7 & 49.9 \\
\hline Iceland & 78.4 & 100.0 & 77.5 & 87.5 & 96.3 & 90.7 & 99.5 & 97.3 & 72.5 & 73.9 & 99.1 & 92.6 & 62.6 & 79.3 & 44.9 & 34.0 & 78.3 & 46.9 \\
\hline India & 48.4 & 69.1 & 30.4 & 52.0 & 61.4 & 30.4 & 70.1 & 58.0 & 59.1 & 21.8 & 77.4 & 27.1 & 53.3 & 63.1 & 39.2 & 35.9 & 58.6 & 15.5 \\
\hline Indonesia & 54.4 & 76.9 & 44.8 & 53.8 & 73.8 & 62.1 & 76.1 & 61.8 & 63.7 & 21.5 & 66.2 & 48.8 & 46.4 & 84.0 & 42.8 & 33.7 & 59.5 & 8.6 \\
\hline Iran, Islamic Rep. & 58.5 & 99.9 & 60.0 & 63.7 & 86.0 & 38.9 & 87.6 & 80.9 & 47.7 & 22.4 & 65.8 & 64.0 & 51.2 & 59.0 & 42.0 & 61.0 & 46.5 & 18.8 \\
\hline Iraq & 50.9 & n.d. & 39.9 & 51.4 & 47.3 & 47.0 & 78.9 & 81.5 & 48.9 & 7.3 & 85.5 & 57.2 & 8.3 & 80.7 & 27.7 & 49.2 & 60.2 & 42.9 \\
\hline Ireland & 76.7 & 100.0 & 82.5 & 85.0 & 90.2 & 69.9 & 94.8 & 86.4 & 72.4 & 62.5 & 76.4 & 90.7 & 61.3 & 75.9 & 57.1 & 74.4 & 78.1 & 47.2 \\
\hline Israel & 72.3 & 100.0 & 54.9 & 87.2 & 87.5 & 73.6 & 92.9 & 88.1 & 75.8 & 61.1 & 63.4 & 73.3 & 75.5 & 72.0 & 59.0 & 36.3 & 62.9 & 65.3 \\
\hline Italy & 70.9 & 100.0 & 67.7 & 84.3 & 87.4 & 67.0 & 97.3 & 87.8 & 60.5 & 51.2 & 71.8 & 81.0 & 76.0 & 78.7 & 42.4 & 64.2 & 55.0 & 33.1 \\
\hline Jamaica & 59.1 & 97.5 & 53.0 & 67.9 & 70.3 & 69.5 & 88.2 & 77.6 & 48.1 & 34.2 & 46.7 & 83.3 & 54.6 & 80.0 & 29.6 & 37.3 & 44.1 & 22.0 \\
\hline Japan & 75.0 & 100.0 & 75.4 & 85.3 & 83.9 & 59.6 & 98.3 & 87.6 & 76.5 & 87.6 & 82.2 & 82.7 & 70.8 & 68.6 & 42.6 & 53.6 & 78.1 & 41.4 \\
\hline Jordan & 62.7 & 99.8 & 56.0 & 68.8 & 80.7 & 38.5 & 89.1 & 86.3 & 52.1 & 27.9 & 73.4 & 69.9 & 62.7 & 84.8 & 27.0 & 63.1 & 68.7 & 17.8 \\
\hline Kazakhstan & 63.9 & 99.9 & 53.4 & 69.8 & 73.1 & 72.4 & 92.6 & 74.0 & 70.5 & 34.6 & 90.3 & 80.2 & 30.5 & 58.2 & n.d. & 55.3 & 50.4 & 16.2 \\
\hline Kenya & 44.0 & 51.1 & 42.5 & 36.3 & 50.0 & 58.6 & 47.6 & 31.6 & 36.9 & 25.7 & 40.9 & 58.8 & 48.7 & 63.7 & 40.3 & 44.0 & 46.5 & 25.3 \\
\hline South Korea & 72.7 & 100.0 & 78.7 & 81.1 & 86.8 & 62.7 & 94.6 & 88.8 & 90.1 & 82.9 & 85.9 & 69.5 & 81.5 & 66.4 & 30.4 & 45.5 & 57.5 & 33.0 \\
\hline Kuwait & 52.5 & 100.0 & 72.7 & 76.4 & 78.6 & 54.7 & 66.0 & 85.1 & 71.4 & 33.2 & n.d. & & 21.5 & 38.2 & 34.5 & 40.1 & 59.2 & 9.0 \\
\hline Kyrgyz Republic & 60.9 & 95.8 & 62.6 & 63.9 & 72.0 & 62.3 & 87.8 & 82.5 & 39.9 & 15.5 & 78.8 & 74.5 & 4.2 & 84.0 & n.d. & 65.7 & 44.7 & 39.7 \\
\hline Lao PDR & 49.9 & 56.4 & 44.7 & 46.2 & 64.3 & 49.0 & 72.4 & 33.4 & 62.5 & 15.1 & 69.9 & 52.4 & 44.7 & 74.5 & n.d. & 42.4 & 53.1 & 17.5 \\
\hline Latvia & 72.5 & 100.0 & 58.2 & 77.3 & 85.3 & 70.2 & 94.8 & 88.2 & 71.2 & 49.3 & 75.0 & 86.0 & 66.4 & 77.8 & 73.5 & 69.2 & 55.4 & 34.5 \\
\hline Lebanon & 58.0 & n.d. & 55.4 & 70.0 & 77.2 & 45.9 & 89.8 & 88.2 & 60.3 & 34.5 & n.d. & 63.5 & 47.7 & 80.9 & 37.2 & 55.0 & 52.3 & 11.9 \\
\hline Lesotho & 45.9 & 13.2 & 48.7 & 28.0 & 46.7 & 72.8 & 61.1 & 23.9 & 25.2 & 12.7 & 28.2 & 73.0 & 46.7 & 75.0 & n.d. & 63.2 & 33.3 & 83.3 \\
\hline Liberia & 30.5 & 0.1 & 34.0 & 20.3 & 11.7 & 35.0 & 52.0 & - & 38.5 & 10.4 & 66.1 & 26.5 & - & 62.6 & 36.7 & 47.6 & 43.6 & 33.2 \\
\hline Lithuania & 72.1 & 100.0 & 65.7 & 80.1 & 87.2 & 71.5 & 94.0 & 74.9 & 70.4 & 48.5 & 67.7 & 85.4 & 63.9 & 85.2 & 61.7 & 77.7 & 51.7 & 40.2 \\
\hline Luxembourg & 76.7 & 100.0 & 64.7 & 85.9 & 65.7 & 73.5 & 99.0 & 64.9 & 74.9 & 69.9 & 85.7 & 84.7 & 77.1 & 48.7 & n.d. & 60.8 & 78.7 & 92.3 \\
\hline Macedonia, FYR & 62.8 & 98.1 & 63.1 & 77.5 & 68.7 & 43.5 & 94.7 & 76.4 & 39.8 & 33.2 & 51.8 & 81.7 & 43.2 & 85.2 & n.d. & 63.5 & 60.7 & 23.0 \\
\hline
\end{tabular}




\begin{tabular}{|c|c|c|c|c|c|c|c|c|c|c|c|c|c|c|c|c|c|c|}
\hline Country & $\begin{array}{l}\text { SDG } \\
\text { Index }\end{array}$ & $\begin{array}{c}\text { SDG } \\
1\end{array}$ & $\begin{array}{c}\text { SDG } \\
2\end{array}$ & $\begin{array}{c}\text { SDG } \\
3\end{array}$ & $\begin{array}{c}\text { SDG } \\
4\end{array}$ & $\begin{array}{c}\text { SDG } \\
5\end{array}$ & $\begin{array}{c}\text { SDG } \\
6\end{array}$ & $\begin{array}{c}\text { SDG } \\
7\end{array}$ & $\begin{array}{c}\text { SDG } \\
8\end{array}$ & $\begin{array}{c}\text { SDG } \\
9\end{array}$ & $\begin{array}{c}\text { SDG } \\
10\end{array}$ & $\begin{array}{c}\text { SDG } \\
11\end{array}$ & $\begin{array}{c}\text { SDG } \\
12\end{array}$ & $\begin{array}{c}\text { SDG } \\
13\end{array}$ & $\begin{array}{c}\text { SDG } \\
14\end{array}$ & $\begin{array}{c}\text { SDG } \\
15\end{array}$ & $\begin{array}{c}\text { SDG } \\
16\end{array}$ & $\begin{array}{c}\text { SDG } \\
17\end{array}$ \\
\hline Madagascar & 36.2 & - & 36.1 & 33.1 & 31.7 & 70.5 & 33.4 & 3.1 & 35.4 & 4.7 & 50.4 & 48.8 & 44.0 & 83.9 & 47.4 & 46.6 & 41.2 & 5.4 \\
\hline Malawi & 39.8 & - & 39.1 & 30.0 & 57.9 & 60.3 & 70.3 & - & 23.5 & 10.9 & 50.9 & 55.0 & 46.6 & 49.8 & n.d. & 61.0 & 37.5 & 43.7 \\
\hline Malaysia & 61.7 & 99.6 & 64.2 & 69.7 & 78.0 & 53.7 & 97.1 & 84.9 & 63.9 & 46.7 & 44.8 & 85.1 & 41.3 & 71.9 & 45.0 & 18.5 & 57.9 & 25.9 \\
\hline Mali & 38.2 & 28.4 & 34.0 & 23.6 & 7.6 & 26.8 & 55.5 & 8.8 & 34.1 & 11.0 & 79.7 & 29.1 & 45.2 & 78.8 & n.d. & 66.1 & 55.1 & 27.7 \\
\hline Malta & 72.0 & 100.0 & 60.0 & 84.8 & 80.6 & 56.6 & 94.0 & 89.3 & 71.2 & 47.2 & n.d. & 85.4 & 53.1 & 71.1 & 65.3 & 57.1 & 66.3 & 69.3 \\
\hline Mauritania & 39.6 & 84.1 & 47.3 & 35.3 & 17.5 & 29.8 & 47.7 & 26.1 & 27.0 & 8.3 & 60.0 & 18.2 & 46.6 & 65.6 & 62.3 & 35.3 & 39.1 & 22.8 \\
\hline Mauritius & 60.7 & 99.2 & 41.8 & 72.0 & 87.0 & 52.2 & 95.0 & 86.6 & 55.0 & 29.6 & n.d. & 90.0 & 32.4 & 85.0 & 42.4 & 28.8 & 57.0 & 17.5 \\
\hline Mexico & 63.4 & 96.1 & 63.6 & 73.2 & 78.2 & 73.2 & 90.2 & 81.2 & 62.8 & 28.9 & 42.3 & 85.5 & 58.4 & 85.5 & 54.4 & 36.6 & 39.6 & 27.7 \\
\hline Moldova & 66.6 & 99.9 & 62.9 & 70.4 & 66.7 & 68.6 & 82.4 & 82.9 & 53.8 & 30.2 & 79.7 & 74.8 & 34.2 & 91.4 & n.d. & 63.1 & 44.1 & 60.6 \\
\hline Mongolia & 58.1 & 99.4 & 49.5 & 65.8 & 80.9 & 68.0 & 60.5 & 37.5 & 66.6 & 17.0 & 70.5 & 55.7 & 55.0 & 67.7 & n.d. & 57.5 & 45.0 & 32.3 \\
\hline Montenegro & 62.5 & 97.5 & 51.6 & 75.6 & 86.8 & 38.8 & 97.4 & 84.9 & 48.8 & 30.3 & 91.5 & 81.5 & 5.1 & 79.6 & 40.6 & 47.8 & 62.8 & 41.4 \\
\hline Morocco & 61.6 & 95.5 & 56.8 & 60.9 & 66.8 & 38.3 & 78.1 & 82.2 & 53.5 & 35.0 & 58.9 & 77.3 & 57.3 & 84.0 & 54.9 & 56.7 & 56.5 & 34.9 \\
\hline Mozambique & 39.5 & - & 28.5 & 20.7 & 40.9 & 56.2 & 36.7 & 35.4 & 27.8 & 7.0 & 46.3 & 52.6 & 51.2 & 69.4 & 73.1 & 52.8 & 38.1 & 34.3 \\
\hline Myanmar & 44.5 & n.d. & 48.1 & 44.8 & 53.9 & 63.6 & 78.9 & 40.3 & 55.2 & 2.4 & n.d. & 26.8 & 47.2 & 73.2 & 31.7 & 49.8 & 43.5 & 8.2 \\
\hline Namibia & 49.9 & 67.1 & 24.4 & 43.6 & 58.0 & 88.0 & 69.0 & 37.9 & 35.8 & 25.0 & - & 75.0 & 53.1 & 73.4 & 54.2 & 49.4 & 49.6 & 44.6 \\
\hline Nepal & 51.5 & 78.3 & 41.4 & 51.3 & 69.7 & 54.8 & 73.4 & 49.9 & 34.7 & 10.9 & 80.3 & 25.7 & 50.0 & 86.5 & n.d. & 50.8 & 44.9 & 22.1 \\
\hline Netherlands & 78.9 & 100.0 & 75.5 & 91.6 & 90.1 & 81.2 & 98.1 & 84.4 & 65.1 & 75.0 & 85.3 & 82.6 & 80.8 & 47.0 & 49.8 & 72.6 & 81.6 & 81.2 \\
\hline New Zealand & 74.0 & 100.0 & 68.7 & 84.3 & 96.6 & 80.8 & 99.8 & 91.9 & 68.8 & 63.0 & 79.0 & 91.1 & 55.6 & 75.1 & 46.4 & 34.7 & 76.3 & 46.6 \\
\hline Nicaragua & 57.4 & 84.2 & 47.0 & 64.7 & 62.8 & 83.4 & 78.9 & 63.9 & 51.2 & 11.9 & 60.0 & 88.2 & 45.1 & 84.8 & 36.8 & 53.6 & 42.3 & 16.8 \\
\hline Niger & 31.4 & 26.8 & 21.4 & 25.9 & - & 14.0 & 38.1 & 1.7 & 35.3 & 2.7 & 75.7 & 29.4 & 46.7 & 49.8 & n.d. & 41.4 & 68.0 & 26.0 \\
\hline Nigeria & 36.1 & 22.2 & 43.5 & 19.2 & 19.8 & 24.2 & 51.3 & 38.8 & 42.1 & 16.4 & 37.9 & 19.5 & 46.6 & 88.2 & 33.4 & 72.8 & 37.3 & \\
\hline Norway & 82.3 & 100.0 & 55.3 & 89.0 & 92.7 & 88.2 & 99.2 & 95.9 & 68.3 & 72.1 & 98.9 & 93.8 & 63.5 & 72.7 & 69.6 & 71.0 & 83.9 & 85.4 \\
\hline Oman & 59.9 & 100.0 & 69.1 & 75.3 & 76.7 & 32.3 & 86.7 & 77.7 & 73.7 & 45.7 & n.d. & 60.4 & 51.4 & 46.4 & 52.4 & 36.6 & 63.6 & 10.1 \\
\hline Pakistan & 45.7 & 87.9 & 28.7 & 41.3 & 22.2 & 25.6 & 73.7 & 62.9 & 41.9 & 14.4 & 87.7 & 31.4 & 45.2 & 82.0 & 28.5 & 59.8 & 41.1 & 2.7 \\
\hline Panama & 60.7 & 95.8 & 53.4 & 68.1 & 72.4 & 64.6 & 86.9 & 81.4 & 72.8 & 32.6 & 29.7 & 91.2 & 50.0 & 87.1 & 45.2 & 42.7 & 33.3 & 24.8 \\
\hline Paraguay & 59.3 & 96.8 & 69.1 & 63.8 & 58.4 & 64.5 & 94.3 & 81.4 & 45.6 & 14.3 & 28.4 & 81.6 & 49.4 & 80.9 & n.d. & 47.4 & 41.0 & 32.4 \\
\hline Peru & 58.4 & 94.6 & 62.2 & 66.9 & 73.6 & 63.3 & 81.9 & 74.6 & 48.0 & 21.7 & 39.7 & 79.4 & 52.4 & 81.3 & 49.6 & 45.1 & 42.9 & 15.3 \\
\hline Philippines & 55.5 & 80.9 & 46.2 & 57.8 & 66.3 & 62.9 & 83.0 & 67.4 & 55.5 & 17.2 & 53.4 & 69.3 & 46.9 & 83.4 & 53.6 & 35.0 & 52.6 & 12.9 \\
\hline Poland & 69.8 & 100.0 & 60.3 & 79.3 & 86.4 & 68.2 & 96.1 & 82.4 & 69.2 & 44.5 & 80.5 & 82.0 & 73.4 & 76.1 & 38.8 & 69.9 & 59.2 & 20.6 \\
\hline Portugal & 71.5 & 100.0 & 47.9 & 83.9 & 87.2 & 80.9 & 98.8 & 90.3 & 68.7 & 52.4 & 77.7 & 89.8 & 65.7 & 84.8 & 53.4 & 35.0 & 63.8 & 35.1 \\
\hline Qatar & 65.8 & 100.0 & 60.6 & 85.4 & 76.4 & 48.1 & 65.9 & 78.8 & 69.5 & 72.1 & 58.3 & 57.2 & 72.4 & 38.2 & 61.4 & 27.3 & 81.7 & n.d. \\
\hline Romania & 67.5 & 100.0 & 60.5 & 73.1 & 70.5 & 60.2 & 91.8 & 83.5 & 67.9 & 32.1 & 94.6 & 65.2 & 48.0 & 86.0 & 75.2 & 65.9 & 48.8 & 24.4 \\
\hline $\begin{array}{l}\text { Russian } \\
\text { Federation }\end{array}$ & 66.4 & 100.0 & 55.0 & 75.4 & 83.2 & 67.7 & 87.2 & 85.2 & 79.6 & 44.5 & 61.0 & 82.0 & 53.3 & 65.8 & 54.2 & 59.7 & 37.2 & 37.1 \\
\hline Rwanda & 44.0 & 12.4 & 36.3 & 48.7 & 55.7 & 76.8 & 69.1 & 4.5 & 42.5 & 15.1 & 32.6 & 44.0 & 46.4 & 74.8 & n.d. & 53.7 & 50.6 & 40.6 \\
\hline Saudi Arabia & 58.0 & 100.0 & 54.4 & 72.4 & 88.8 & 39.3 & 64.6 & 82.6 & 72.7 & 50.7 & n.d. & & 53.3 & 54.1 & 51.4 & 62.4 & 63.7 & 18.1 \\
\hline Senegal & 45.8 & 44.7 & 47.3 & 42.6 & 21.7 & 46.8 & 65.1 & 51.4 & 39.6 & 18.0 & 60.5 & 47.0 & 47.4 & 76.2 & 35.4 & 58.2 & 54.7 & 22.6 \\
\hline Serbia & 68.3 & 99.9 & 70.0 & 75.0 & 80.8 & 56.5 & 97.9 & 78.4 & 44.1 & 35.6 & 88.7 & 81.7 & 48.3 & 81.1 & n.d. & 62.1 & 56.1 & 36.5 \\
\hline Sierra Leone & 36.9 & 23.9 & 33.6 & 18.2 & 24.9 & 22.9 & 41.8 & 2.4 & 29.8 & 5.4 & 73.6 & 28.1 & 47.1 & 74.6 & 50.9 & 73.1 & 49.1 & 28.4 \\
\hline Singapore & 74.6 & 100.0 & 67.3 & 85.2 & 89.5 & 68.7 & 100.0 & 89.6 & 74.5 & 80.6 & n.d. & 82.8 & 87.1 & 81.8 & 32.1 & 49.3 & 80.5 & 24.9 \\
\hline Slovak Republic & 72.7 & 100.0 & 73.3 & 81.8 & 74.1 & 68.4 & 99.4 & 88.1 & 64.4 & 48.4 & 98.3 & 81.3 & 67.2 & 82.1 & n.d. & 59.2 & 54.6 & 22.6 \\
\hline Slovenia & 76.6 & 100.0 & 65.6 & 87.2 & 90.4 & 80.1 & 99.1 & 90.2 & 66.3 & 58.2 & 84.7 & 85.5 & 66.9 & 79.4 & 74.1 & 70.3 & 66.6 & 37.8 \\
\hline South Africa & 53.8 & 75.9 & 60.7 & 41.9 & 72.7 & 81.4 & 80.5 & 74.5 & 29.0 & 42.5 & - & 80.8 & 46.4 & 66.0 & 44.0 & 34.7 & 41.8 & 41.5 \\
\hline Spain & 72.2 & 100.0 & 51.7 & 88.8 & 93.2 & 81.7 & 97.3 & 90.7 & 57.3 & 62.4 & 75.4 & 88.0 & 77.7 & 76.9 & 44.0 & 47.9 & 64.3 & 30.2 \\
\hline Sri Lanka & 54.8 & 97.5 & 34.7 & 69.4 & 77.6 & 50.9 & 93.0 & 59.5 & 66.4 & 21.4 & 70.8 & 67.8 & 3.3 & 79.8 & 43.9 & 34.7 & 60.1 & 0.9 \\
\hline
\end{tabular}


On metrics and financing for the Sustainable Development Goals

\begin{tabular}{|c|c|c|c|c|c|c|c|c|c|c|c|c|c|c|c|c|c|c|}
\hline Country & $\begin{array}{l}\text { SDG } \\
\text { Index }\end{array}$ & $\begin{array}{c}\text { SDG } \\
1\end{array}$ & $\begin{array}{l}\text { SDG } \\
2\end{array}$ & $\begin{array}{c}\text { SDG } \\
3\end{array}$ & $\begin{array}{c}\text { SDG } \\
4\end{array}$ & $\begin{array}{l}\text { SDG } \\
5\end{array}$ & $\begin{array}{l}\text { SDG } \\
6\end{array}$ & $\begin{array}{l}\text { SDG } \\
7\end{array}$ & $\begin{array}{c}\text { SDG } \\
8\end{array}$ & $\begin{array}{l}\text { SDG } \\
9\end{array}$ & $\begin{array}{c}\text { SDG } \\
10\end{array}$ & $\begin{array}{c}\text { SDG } \\
11\end{array}$ & $\begin{array}{c}\text { SDG } \\
12\end{array}$ & $\begin{array}{c}\text { SDG } \\
13\end{array}$ & $\begin{array}{c}\text { SDG } \\
14\end{array}$ & $\begin{array}{c}\text { SDG } \\
15\end{array}$ & $\begin{array}{c}\text { SDG } \\
16\end{array}$ & $\begin{array}{c}\text { SDG } \\
17\end{array}$ \\
\hline Sudan & 42.2 & 78.3 & 11.9 & 41.6 & 40.0 & 40.4 & 34.5 & 35.5 & 33.1 & 12.7 & 73.7 & 45.0 & 43.9 & 67.6 & 40.4 & 63.4 & 55.0 & \\
\hline Suriname & 58.0 & n.d. & 62.3 & 63.5 & 71.6 & 64.0 & 88.5 & 93.7 & 59.8 & 26.1 & 27.2 & 75.9 & 38.5 & 55.7 & 50.1 & 69.9 & 62.6 & 18.4 \\
\hline Swaziland & 45.1 & 38.9 & 44.8 & 34.7 & 58.3 & 61.7 & 64.3 & 38.7 & 36.9 & 19.4 & 30.8 & 75.8 & 74.2 & 49.2 & n.d. & 32.1 & 29.8 & 32.5 \\
\hline Sweden & 84.5 & 100.0 & 69.6 & 89.6 & 85.0 & 87.5 & 99.6 & 96.1 & 64.4 & 85.1 & 100.0 & 92.4 & 80.1 & 82.8 & 65.9 & 66.0 & 81.5 & 91.4 \\
\hline Switzerland & 80.9 & 100.0 & 69.7 & 89.0 & 75.4 & 76.7 & 99.6 & 92.9 & 73.0 & 77.4 & 78.0 & 81.8 & 75.2 & 86.3 & n.d. & 69.5 & 85.2 & 64.2 \\
\hline Tajikistan & 60.2 & 93.1 & 34.9 & 60.6 & 73.2 & 52.9 & 75.8 & 86.2 & 48.4 & 13.3 & 85.5 & 70.4 & 44.0 & 70.2 & n.d. & 67.1 & 60.1 & 26.9 \\
\hline Tanzania & 43.0 & 32.2 & 34.3 & 39.4 & 40.5 & 65.8 & 37.4 & 20.1 & 48.5 & 10.3 & 67.7 & 52.0 & 49.1 & 81.2 & 46.3 & 40.0 & 38.3 & 28.0 \\
\hline Thailand & 62.2 & 99.9 & 53.5 & 62.9 & 77.6 & 66.0 & 94.7 & 77.5 & 72.0 & 35.6 & 62.9 & 64.0 & 42.7 & 58.9 & 50.9 & 59.2 & 49.3 & 29.2 \\
\hline Togo & 40.9 & 21.2 & 42.7 & 35.6 & 54.5 & 22.4 & 41.6 & 12.3 & 33.7 & 6.2 & 63.1 & 27.3 & 46.4 & 79.5 & 48.7 & 72.3 & 58.6 & 28.4 \\
\hline $\begin{array}{l}\text { Trinidad and } \\
\text { Tobago }\end{array}$ & 59.1 & 100.0 & 50.8 & 67.8 & 75.6 & 69.7 & 92.7 & 76.6 & 62.9 & 31.8 & n.d. & 81.7 & 2.6 & 44.7 & 40.7 & 47.6 & 40.4 & n.d. \\
\hline Tunisia & 65.1 & 97.1 & 56.1 & 68.8 & 84.5 & 51.4 & 89.0 & 86.8 & 48.7 & 29.8 & 71.7 & 80.2 & 57.6 & 85.0 & 50.3 & 59.1 & 55.4 & 34.6 \\
\hline Turkey & 66.1 & 99.6 & 64.8 & 74.2 & 81.2 & 42.7 & 96.4 & 87.7 & 63.9 & 42.8 & 61.2 & 82.2 & 59.1 & 84.2 & 46.5 & 51.8 & 56.1 & 29.5 \\
\hline Uganda & 43.6 & 51.6 & 37.5 & 36.4 & 57.8 & 61.7 & 55.0 & 4.6 & 43.1 & 16.1 & 49.9 & 41.0 & 45.5 & 89.2 & n.d. & 46.5 & 37.4 & 24.8 \\
\hline Ukraine & 66.4 & 100.0 & 72.7 & 70.9 & 85.9 & 64.4 & 95.1 & 86.1 & 56.8 & 24.9 & 99.3 & 77.1 & 14.7 & 79.8 & 55.0 & 56.4 & 39.4 & 50.0 \\
\hline $\begin{array}{l}\text { United Arab } \\
\text { Emirates }\end{array}$ & 63.6 & 100.0 & 44.0 & 79.5 & 71.6 & 64.5 & 65.5 & 83.3 & 73.0 & 67.9 & n.d. & 15.3 & 66.0 & 45.8 & 60.7 & 40.8 & 75.7 & n.d. \\
\hline United Kingdom & 78.1 & 100.0 & 78.1 & 84.7 & 87.5 & 81.3 & 99.1 & 87.0 & 76.2 & 72.5 & 71.9 & 88.8 & 82.8 & 76.5 & 49.8 & 46.6 & 78.7 & 67.0 \\
\hline United States & 72.7 & 100.0 & 88.5 & 80.2 & 75.1 & 74.4 & 98.2 & 87.6 & 82.5 & 81.8 & 59.1 & 88.4 & 61.5 & 54.0 & 45.2 & 44.3 & 60.9 & 54.3 \\
\hline Uruguay & 68.0 & 100.0 & 71.9 & 76.5 & 88.8 & 70.8 & 98.2 & 92.7 & 62.9 & 33.8 & 47.2 & 93.1 & 49.0 & 86.6 & 44.4 & 36.0 & 57.1 & 47.4 \\
\hline Venezuela, RB & 61.8 & 100.0 & 58.7 & 72.4 & 76.3 & 65.6 & 93.1 & 87.0 & 50.8 & 31.4 & 48.6 & 81.0 & 44.6 & 78.1 & 24.0 & 55.6 & 21.7 & n.d. \\
\hline Vietnam & 57.6 & 95.3 & 61.0 & 64.1 & 76.0 & 72.4 & 89.2 & 70.8 & 57.4 & 32.6 & 73.0 & 53.1 & 34.4 & 63.7 & 33.8 & 33.9 & 52.7 & 16.1 \\
\hline Yemen, Rep. & 37.3 & n.d. & 18.5 & 45.6 & 47.7 & 7.7 & 36.7 & 44.2 & 23.1 & 7.6 & 67.4 & 25.2 & 0.5 & 92.5 & 51.8 & 65.5 & 45.6 & 17.5 \\
\hline Zambia & 38.4 & 6.3 & 27.3 & 35.8 & 55.3 & 57.9 & 55.2 & 39.9 & 27.0 & 13.4 & 15.0 & 53.8 & 48.2 & 73.2 & n.d. & 59.0 & 37.7 & 9.4 \\
\hline Zimbabwe & 48.6 & n.d. & 31.7 & 34.7 & 65.3 & 72.3 & 58.8 & 42.1 & 40.9 & 16.6 & n.d. & 76.6 & 51.7 & 76.0 & n.d. & 56.6 & 34.6 & 22.7 \\
\hline
\end{tabular}


Table 16 | Sources and summary statistics of variables in partial correlation matrix Partial correlation coefficients are reported in Table 3. The dependent variable is the Cantril Ladder.

\begin{tabular}{|c|c|c|c|c|c|c|}
\hline Variable & Source & $\begin{array}{l}\text { Number } \\
\text { of obs. }\end{array}$ & Mean & $\begin{array}{l}\text { Standard } \\
\text { Deviation }\end{array}$ & Min & Max \\
\hline $\begin{array}{l}\text { Cantril Ladder of Subjective Well- } \\
\text { being, 0-10 (2016) }\end{array}$ & $\begin{array}{l}\text { Helliwell et al. } \\
\text { (2016a) }\end{array}$ & 119 & 5.44 & 1.13 & 2.70 & 7.60 \\
\hline $\begin{array}{l}\text { Sustainable Development Goals } \\
\text { Index, corrected for Subjective } \\
\text { Wellbeing, } 0-100 \text { (2016) }\end{array}$ & $\begin{array}{l}\text { Sachs et al. } \\
(2016 b)\end{array}$ & 119 & 60.67 & 13.19 & 30.5 & 84.72 \\
\hline $\begin{array}{l}\text { Global Competitiveness Index, 0- } \\
100(2015-2016)\end{array}$ & WEF (2016) & 119 & 4.24 & 0.65 & 2.84 & 5.76 \\
\hline $\begin{array}{l}\text { Human Development Index, 0-100 } \\
(2016)\end{array}$ & UNDP (2016a) & 119 & 0.72 & 0.15 & 0.39 & 0.94 \\
\hline $\begin{array}{l}\text { Index for Economic Freedom, 0- } \\
100(2016)\end{array}$ & Miller et al. (2016) & 119 & 62.77 & 8.73 & 38.20 & 81.60 \\
\hline $\begin{array}{l}\text { Log GDP per capita in constant } \\
2011 \text { international dollars, PPP } \\
(2015)\end{array}$ & $\begin{array}{l}\text { World Bank } \\
(2017 \mathrm{c}), \text { Helliwell et } \\
\text { al.. (2016a) }\end{array}$ & 119 & 9.30 & 1.13 & 6.60 & 11.43 \\
\hline $\begin{array}{l}\text { Unemployment, \% total labor force } \\
\text { (2014) }\end{array}$ & $\begin{array}{l}\text { ILO (2016b), Miller } \\
\text { et al. (2016) }\end{array}$ & 119 & 8.45 & 6.16 & 0.40 & 31.00 \\
\hline
\end{tabular}

\section{Table 17| Significant partial correlates of subjective well-being}

Indicators included in the SDG Index that are partially correlated $(p<0.05)$ with SWB in the presence of GDP per capita (PPP) and unemployment as control variable. The third column identifies the indicators that were jointly significant in the presence of the control variable following stepwise backward elimination of non-significant partial correlates.

\begin{tabular}{|l|l|l|}
\multicolumn{1}{|c|}{ SDG } & \multicolumn{1}{|c|}{ Indicator } & \multicolumn{1}{c|}{$\begin{array}{c}\text { Jointly } \\
\text { significant? }\end{array}$} \\
\hline 3 & Healthy Life Expectancy at birth (years) & no \\
\hline 3 & Traffic deaths rate (per 100,000 people) & no \\
\hline 3 & Incidence of tuberculosis (per 100,000 people) & no \\
\hline 5 & Proportion of seats held by women in national parliaments (\%) & yes \\
\hline 7 & CO2 emissions from fuel combustion / electricity output (MtCO2/TWh) & yes \\
\hline 9 & Proportion of the population using the internet (\%) & yes \\
\hline 11 & Improved water source, piped (\% of urban population with access) & no \\
\hline 12 & Percentage of anthropogenic wastewater that receives treatment (\%) & no \\
\hline 16 & Corruption Perception Index (0-100) & no \\
\hline 17 & Government Health, Education and R\&D spending (\%GDP) & no \\
\hline
\end{tabular}




\section{On metrics and financing for the Sustainable Development Goals}

This chapter is based on the paper: Fankhauser S, G. Schmidt-Traub. 2011, "From adaptation to climate-resilient development: the costs of climate-proofing the Millennium Development Goals in Africa," Climate and Development 3: 94-113. 


\section{Chapter 3}

\section{From adaptation to climate-}

resilient development: The costs of climate-proofing the

\section{Millennium Development Goals}

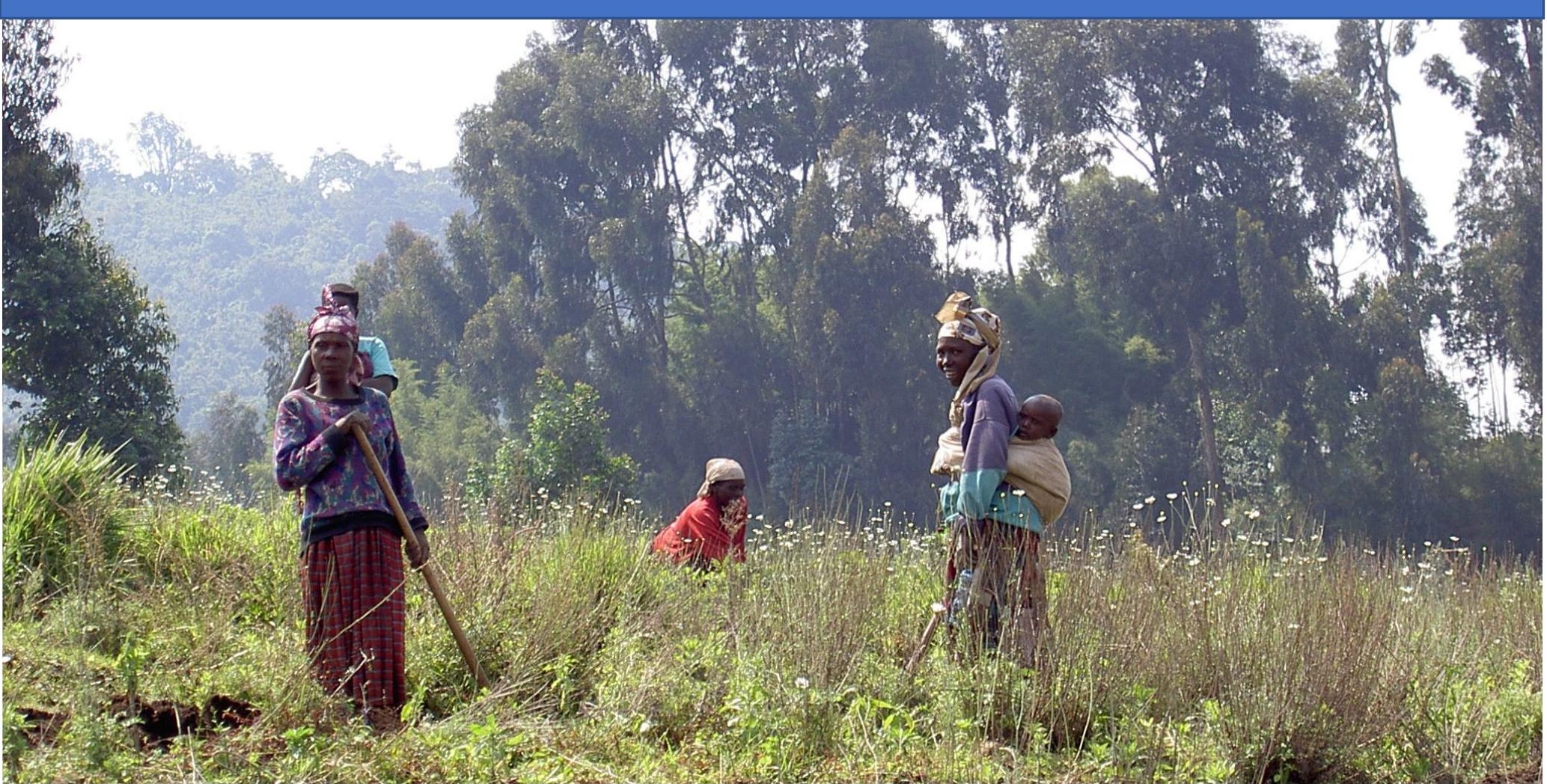


On metrics and financing for the Sustainable Development Goals

Socio-economic development and adaptation to climate change are closely intertwined. Adaptation is sometimes described as climate-resilient development or development under a hostile climate. In support of this view, this chapter estimates the combined cost of meeting and at the same time 'climate-proofing' the Millennium Development Goals (MDGs) for Africa. Treating adaptation and development in an integrated way helps to better understand financing requirements analytically and, more importantly, to implement the requisite measures more effectively as part of an integrated development program. We find that the external financing needed for 'climate-resilient' MDGs is about 40\% higher than the external financing for the MDGs alone, up to US\$100 billion a year for the next decade, compared with US\$72 billion a year for the MDGs alone. This estimate is indicative only and based on fairly cursory aggregate data. The next challenge would be to apply the integrated adaptation and development frameworks to concrete development strategies at the country level. 


\subsection{Introduction}

Economic development and adaptation to climate change are closely linked nowhere more so than in Africa. Africa faces the biggest development challenges of any continent (Sachs et al., 2004a; Commission for Africa, 2005). It is also one of the most vulnerable places to climate change anywhere in the world (Barr et al., 2010; World Bank, 2010a), even though it has contributed little to the problem. Among the most prominent impacts that may affect the continent are: an increase in the number of people at risk of water stress; an increase in exposure to malaria; a drop in agricultural yields; and rising sea levels that affect mangrove forests as well as coastal fisheries, and lead to increased flooding (Boko et al., 2007; Stern, 2007; Collier et al., 2008; Müller, 2009; UNCCD, 2009).

There is a growing awareness, both in the literature and among development practitioners, of the strong connection between adaptation and development. The World Bank (2010b) calls for more climate-resilient development. Stern (2009b) simply defines adaptation as 'development in a more hostile climate'.

McGray et al. (2007) and Klein and Persson (2008) argue that vulnerability to climate change can be reduced through measures that range from pure development to pure adaptation. Fankhauser and Burton (2011) put development-style projects at the core of spending adaptation finance wisely. Benson and Clay (1998), Dell et al. (2009), Noy (2009), and Vivid Economics (2010), among others, discuss how different growth paths both affect and are affected by climate vulnerability. Adaptive capacity - the ability to deal with climate events - is known to be driven predominantly by indicators of development such as health, literacy, income and institutional quality (Tol and Yohe, 2007; Barr et al., 2010).

Yet, in much of the policy discourse adaptation is still treated as a stand-alone issue with little or no links to other development challenges. For example, the negotiations on climate change finance have largely treated financing for climate change adaptation as distinct from development finance and falling under the purview of Ministers of the Environment (Schmidt-Traub, 2009; AGF, 2010). Analyses of adaptation needs tend to see adaptation as an incremental activity bolted onto a 'business as usual' development path (UNFCCC, 2007; Agrawala and Fankhauser, 2008; Parry et al., 2009; World Bank, 2010a), and the same applies to adaptation finance (Müller, 2008; Atteridge et al., 2009; World Bank, 2010b).

The main practical instrument to advance adaptation planning in least developed countries under the UN Framework Convention on Climate Change (UNFCCC), the National Adaptation Programs of Action (NAPAs), were developed in parallel to national development strategies, poverty reduction strategies and associated medium-term expenditure frameworks (MTEFs) that form the basis for providing and programming international development assistance (Osman-Elasha and Downing, 2007). Although initiatives like the Pilot Program for Climate Resilience aim to change this, competing interests within governments often mean that adaptation is seen as an 'environmental' issue to be kept separate from financing for 'development'.

The aim of this chapter is to improve our analytical understanding of adaptation as climate-resilient development, using Africa as an example. Development assistance has many facets but for the purpose of this illustration we focus on the costs of 'climateproofing' the millennium development goals (MDGs). In line with the MDG Africa Steering Group (Ki-Moon et al., 2008), we treat the MDGs as broad outcome goals and include key inputs that are needed to meet the MDGs. For example, we include investments in secondary education, agricultural productivity, transport and energy services 
since they are critical inputs for achieving the MDGs, even though no MDG refers to them directly.

We underscore that our Africa-wide results are indicative and require considerable refinement at the country level before they can guide policy. Particularly in the case of adaptation, estimates of resource needs are derived from a literature that has wellknown analytical shortcomings (Parry et al., 2009; Fankhauser, 2010), which we do not aim to address in this chapter.

Our contribution is to combine the analysis of financing needs for development in the absence of climate change, as approximated by the MDGs, with that for climate change adaptation, and arrive at an integrated framework. We believe that, data quality notwithstanding, this approach is methodologically sound. Gradual refinements of our numbers and the filling of remaining analytical gaps will over time lead to a better assessment of the resources Africa requires to meet the MDGs and adapt to climate change.

Some authors will argue that the integration of development and adaptation should go one step further and also include mitigation, for a comprehensive, low-carbon, climateresilient development strategy (Hulme and Neufeldt, 2010). We do not disagree, but to keep the problem tractable our focus is on adaptation and development. Moreover, adaptation and the MDGs rely more heavily on public finance (Ki-Moon et al., 2008) than mitigation, where private finance has an important role to play (AGF, 2010). Stern (Stern, 2009a) provides first pointers on how the inclusion of mitigation may change development priorities. A rapidly growing policy literature describes the potential for mitigation measures in Africa and how they can be co-financed through carbon markets (Schmidt-Traub and Wylie, 2009; UNCCD, 2009).

The chapter is structured as follows. Section 3.2 recapitulates the reasons why integrating adaptation and development is important. Section 3.3 outlines our method to estimate the combined costs of climate-resilient MDGs in Africa. Section 3.4 details investment needs for achieving the MDGs and adapting to climate change by sector and arrives at indicative financing needs. Section 3.5 concludes the chapter.

\subsection{Why adaptation and development must be integrated}

The separation between 'development' and 'adaptation' is understandable for political reasons, since governments aim to distinguish (baseline) development finance from (additional) adaptation finance. Raising adaptation and development finance separately and keeping funds in separate 'pots' makes sense if the aim is to ascertain additionality of funding. ${ }^{1}$ However, from a practical point of view this artificial distinction has important implications for governments and their ability to implement effective adaptation programs.

At the operational level, a strict distinction between adaptation and development ignores the obvious overlaps between the two notions. It is increasingly understood that economic development and growth will change the profile of climate risks and the vulnerability of developing countries. Generally, but not always, development will mean lower vulnerability to climate change (Vivid Economics, 2010).

\footnotetext{
${ }^{1}$ For a broader discussion of governance issues related to adaptation finance see Klein and Möhner (2009)
} 
McGray et al. (2007) and Klein and Persson (2008) talk about a continuum of measures between development and adaptation that can reduce vulnerability (Gupta et al., 2010). It starts with pure development measures like education, health and sanitation that reduce vulnerability to external pressure more broadly (i.e. not just climaterelated stress) and ends with dedicated measures to address anthropogenic climate change, such as sea walls and the development of new crops. In between there are hybrid measures such as the creation of 'response capacity' (e.g. better resource management and institutions) and the management of current climate risks (e.g. disaster preparedness) that are neither pure adaptation nor pure development.

Bad sequencing and insufficient coordination between the different elements in this continuum are likely to reduce the effectiveness of adaptation and will increase transaction costs. For example, it would be nonsensical to structure the distribution of additional insecticide-treated bed nets in new malaria risk areas as a stand-alone adaptation project that is distinct from existing national bed net distribution campaigns. Wherever possible, adaptation programs need to be developed and implemented as part of existing sector strategies. The synergies are substantial, although they are hard to quantify in monetary terms.

As can be seen from the NAPAs, treating adaptation as a separate issue also encourages project-based design and implementation of adaptation measures. Yet, many adaptation challenges are best addressed at a programmatic level, or as a minimum require integration into a broader development framework. The high transaction costs resulting from the small-scale implementation of project-based adaptation measures will yield inferior results, constrain scalability and limit synergies with the development agenda (e.g. on capacity building and 'soft' adaptation). An integrated assessment would allow a clearer allocation of responsibilities for core 'development' and 'adaptation' measures among line ministries. It would also permit the development of integrated macro-economic management strategies and MTEFs that are vital for ensuring the sound results-based programming of public expenditure and maintaining macroeconomic stability (Schmidt-Traub, 2009).

Similarly, unless adaptation measures are integrated into countries' expenditure and macroeconomic frameworks it will be difficult for finance ministries and central banks to manage the increased inflow of foreign currencies once adaptation finance is starting to flow (IMF, 2008). Only by integrating adaptation measures firmly into a country's development framework can sound macro-economic management strategies be developed that are necessary for ministries of finance and the IMF to support large-scale increase in external finance.

The lack of integration between adaptation and development implies that estimates of adaptation costs and funding needs are incomplete and subject to arbitrary delineations on where development ends and adaptation begins. For example, in one of the better-known estimates of resource needs for adaptation (UNDP, 2007) about half of the costs arise from social protection programs, such as cash transfer or employment schemes that mitigate the adverse social impacts of climate shocks. Such schemes can be very powerful in reducing vulnerability to climate change, but they also make good development sense (Carter et al., 2007). Both by building response capacity and dealing with current climate variability they fall into the grey zone between adaptation and development. The United Nations Development Program (UNDP) chose to include social protection interventions in its estimate, but most other studies of resource needs, (e.g. UNFCCC (2007), (2010a) chose to omit such programs.

The absence of a clear analytical distinction between adaptation and development strikes at the heart of the current debate about financing for climate change adaptation. 
A broad consensus exists that funding from rich countries for climate change adaptation in vulnerable developing countries should be 'additional' to development finance (World Bank, 2010b). But without delineating clearly between the financing needs for development and adaptation, it becomes impossible to determine how much additional funding is required for adaptation.

\subsection{An analytical approach to integrating adaptation and development}

\subsubsection{Earlier estimates of adaptation and development costs}

The starting point of our analysis is a baseline estimate of future development needs in Africa in the absence of significant changes to the climate. Such estimates can be derived from available analyses of what it will take to achieve the MDGs, the world's shared goals for addressing extreme poverty in all its forms.

When the MDGs were conceived a decade ago, insufficient attention was paid to climate change. At the time, several governments resisted the inclusion of climate change and its consequences on development into the Millennium Declaration (United Nations, 2000) from which the MDGs were extracted in 2001. Whether intended or not, the discussion on the MDGs has largely assumed that Africa and other developing regions would experience stable climatic conditions. As a result, existing estimates of the cost of achieving the MDGs do not include the additional requirement for adaptation or provisions for a more hostile climate. Similarly, sectoral analyses of the cost of achieving individual MDG objectives generally do not include the additional resource needs for adapting to a changing climate.

The first detailed cost estimates for meeting the MDGs were developed by the UN Millennium Project (Sachs et al., 2004a, 2004b; UN Millennium Project, 2005; Bahadur et al., 2006). Building on earlier back-of-the-envelope estimates (Devarajan et al., 2002) the UN Millennium Project adopted a bottom-up approach that aggregates the resource needs for individual interventions to achieve the MDGs in each country. Where possible, these authors built on available sectoral needs assessment approaches that employed an intervention-based approach (Jones et al., 2003).

An alternative approach to estimating resource needs uses general equilibrium macroeconomic models, which express sectoral investment functions in a highly aggregate form. These models can be used to understand the interaction across sectors within an economy and to factor in economy-wide constraints, for example, in the labor market. Economists at the World Bank have developed much of this latter work (Bourguignon et al., 2008; Lofgren and Diaz-Bonilla, 2008).

Both approaches have been praised and criticized for their methodological assumptions and use of data. As far as Africa is concerned, though, recent estimates of MDG resource needs show a remarkable degree of convergence (Commission for Africa, 2005; UN Millennium Project, 2005; Ki-Moon et al., 2008). While this does not necessarily signify a reduction in uncertainty, it suggests that the discussion may move from the question of how much money is required to how support should be programmed and how the extra resources relate to other spending needs.

There is no such convergence yet on adaptation costs. A 2008 survey by the Organization for Economic Co-operation and Development (OECD) found that outside coastal protection and some case study evidence, very little is known about sectorlevel adaptation costs (Agrawala and Fankhauser, 2008). Although there has been 
further analysis since (World Bank, 2010a), the range of available numbers remains wide. One emerging lesson is that top-down approaches aimed at estimating global adaptation expenditure have severe flaws and the debate around adaptation estimates is now moving towards bottom-up intervention-based approaches, just like the earlier debate on the MDGs.

In the absence of more detailed bottom-up estimates, this chapter relies on aggregate estimates. In particular, we draw heavily on sector-by-sector assessments conducted by the UNFCCC (2007) and the World Bank (2010a), as well as work by Project Catalyst (2009). These studies were reviewed (Fankhauser, 2010), although imperfect, provide reasonable sectoral and regional breakdowns. Several resource estimates focus specifically on Africa (AMCEN, 2008; Stern, 2009a). Muller (2007) includes estimates for urban water management in Africa, an issue that does not receive enough attention in some of the other studies.

\subsubsection{The method for integration}

We structure our analysis around the expenditure tables prepared by the MDG Africa Steering Group (Ki-Moon et al., 2008) ${ }^{2}$ (Table 18). The results presented by the MDG Africa Steering Group are the most recent estimates (at the time of writing) for the cost of achieving the MDGs in Africa. The estimates are derived from sector-level analyses and draw on the combined research and operational expertise of the African Development Bank, European Union, IMF, OECD, United Nations organizations and World Bank.

From an analytic perspective, the results presented by Ki-Moon et al. (2008) offer several advantages. For example, the results are:

- Scalable. The MDG Africa Steering Group estimates cover the entire Africa region, but the sector-by-sector analysis and presentation can be scaled down to national levels, as has indeed been done by the IMF in cooperation with the United Nations (IMF, 2008). These later analyses estimate what it would take to achieve the MDGs at a national level.

- Macroeconomically sound. The IMF analysis in support of the 'Gleneagles Scenarios' shows that it is possible to meet the macro-economic challenges of maintaining stable exchange rates and controlling inflation in the face of a massive increase in public investment that is largely externally financed. ${ }^{3}$

- Sector based. The results are presented by sector, which is how governments are organized. This makes it possible to determine how much funding is required for key line ministries, and how such financing could best be programmed, executed and monitored at international, national and local levels.

\footnotetext{
2 The MDG Africa Steering Group brought together the heads of the major international development organizations under the leadership of UN Secretary-General Ban Ki-Moon to consolidate a consensus view on how the MDGs can be achieved in Africa. Its report and findings are available online at www.mdgafrica.org.

${ }^{3}$ We recognize the deficiencies of MDG needs assessments that rely on adding up sectoral investment needs without integration into a general equilibrium model (Bourguignon et al., 2008). Yet, subsequent IMF analyses on the macroeconomics of implementing the 'Gleneagles Scenarios' in a number of African countries (IMF, 2008) show that the core macroeconomic issues can be addressed and that the overall results presented by the MDG Africa Steering Group are sound.
} 
Critically, a sector-by-sector approach also minimizes the risk of double-counting interventions or leaving important gaps in the analysis. At the same time, it becomes easier to update elements of the analysis in the light of improved data.

- Benchmarked. The analysis uses the MDGs as (reasonably) well-defined objectives that serve as benchmarks that can be used to track progress and the effectiveness of interventions.

Next, we identify and quantify key 'baseline' development interventions that have been omitted from the analysis of the MDG Africa Steering Group. These include, inter alia, the cost of humanitarian assistance, disaster reduction and some capacity development. We then expand the analysis of the MDG Africa Steering Group to introduce climate change and adaptation. We do this by first identifying qualitatively how countries need to revise and expand their development strategies using interventions that fall into three categories:

- 'More of the same' at the country level. In our assessment, the clear majority of spending needs for climate change adaptation covers known and proven interventions that will need to be supplied in greater number (quantity effect, e.g. more bed nets against infectious diseases, more investment in water storage) and/or higher cost (price effect, e.g. higher and properly enforced construction standards to withstand more extreme weather events).

- New interventions at country level. In some instances, countries will need to invest in new types of interventions, such as climate monitoring and forecast systems or sea walls to protect against rising sea levels. Similarly, some activities may have to be carried out differently, for example measures to boost agricultural productivity will have to consider changes in climate and hydrology.

- Regional and global goods. Finally, key investments needs must be undertaken at regional and global levels (e.g. trans-boundary ecosystem management, water management and regional agricultural research).

Using available studies on the resource needs for climate change adaptation and our own analysis we then provide first estimates for the incremental resources required to finance these interventions. Where important gaps exist in cost estimates, these are highlighted together with suggestions for how they can be closed.

The time frame for our analysis is the coming decade, that is, the period 2010-2020. This is somewhat longer than the traditional MDG time frame, but shorter than for most adaptation needs assessments. To reconcile the two time frames, we attempt an outline of how development expenditure may evolve over the medium term. Adaptation estimates were scaled back to 2010-2020 where we felt this was appropriate. Much adaptation is longer term, and needs will evolve. Yet, the overlaps with development are arguably strongest in the short and medium term.

\subsubsection{Data issues and limitations}

The estimates on resource needs cited in this chapter are the best available. Yet, they are highly aggregated and derived from a literature that has well-known analytical shortcomings. This is particularly the case for adaptation cost estimates, which have been criticized as being inaccurate and incomplete by Agrawala and Fankhauser (2008), Parry et al. (2009), and Fankhauser (2010). Analytical gaps are identified by Parry et al. (2009) in terms of: 
3 - The costs of climate proofing the Millennium Development Goals in Africa

- Scope: not all relevant impacts and countries are covered.

- Depth: for a given impact / country not all relevant adaptation options and needs are considered.

- Costing: not all relevant costs are included (e.g. maintenance costs).

- Uncertainty: estimates ignore the cost of dealing with uncertain climate change.

Parry et al. (2009) conclude that the overall effect of the omissions is to underestimate the true resource needs for adaptation, perhaps by as much as a factor of 2 or 3 in the case of the widely used UNFCCC (2007) study. Some of the methodological problems have at least in part been addressed by World Bank (2010a), but many others are inherent to top-down analysis. ${ }^{4}$

Over time, detailed bottom-up assessments should emerge at the country level to provide more clarity. In the meantime, we use available top-down evidence to demonstrate the overall approach, while acknowledging that Africa is too large and too diverse for meaningful analysis at the macro-level.

To recognize uncertainty, resource cost estimates are presented as a range, which was derived from using alternative cost studies and parameter assumptions. However, we do not claim that the results reported here represent the full range of uncertainty. Given the quality of the underlying data, a much wider range would not seem unreasonable.

\subsection{Estimating the cost of climate-resilient development in Africa}

Our cost estimates for climate-resilient MDGs are presented over three tables. The summary results both for MDG and adaptation are shown in Table 18. A sector-bysector tabulation of the main development and adaptation investments required is provided in the Annex. Table 18 contains details of the adaptation needs assessments and the assumptions made. For more information about the MDG baseline costs, readers are referred to UN Millennium Project (2005) and Ki-Moon et al. (2008).

\footnotetext{
${ }^{4}$ Note that World Bank (2010a) claims to provide an upper boundary of estimated costs, contrary to Parry et al. (2009). This may be correct for the sectors covered by the World Bank, but that coverage is incomplete.
} 
On metrics and financing for the Sustainable Development Goals

Table 18 | Overview of cost estimates (\$bn p.a. over the period 2010-2020)

* MDG costs will be met in part from national government budgets. For example, in the case of agriculture ODA is assumed to cover $\$ 8.8$ billion and African governments $\$ 3.4$ billion of a total budget of $\$ 12.2$ billion a year.

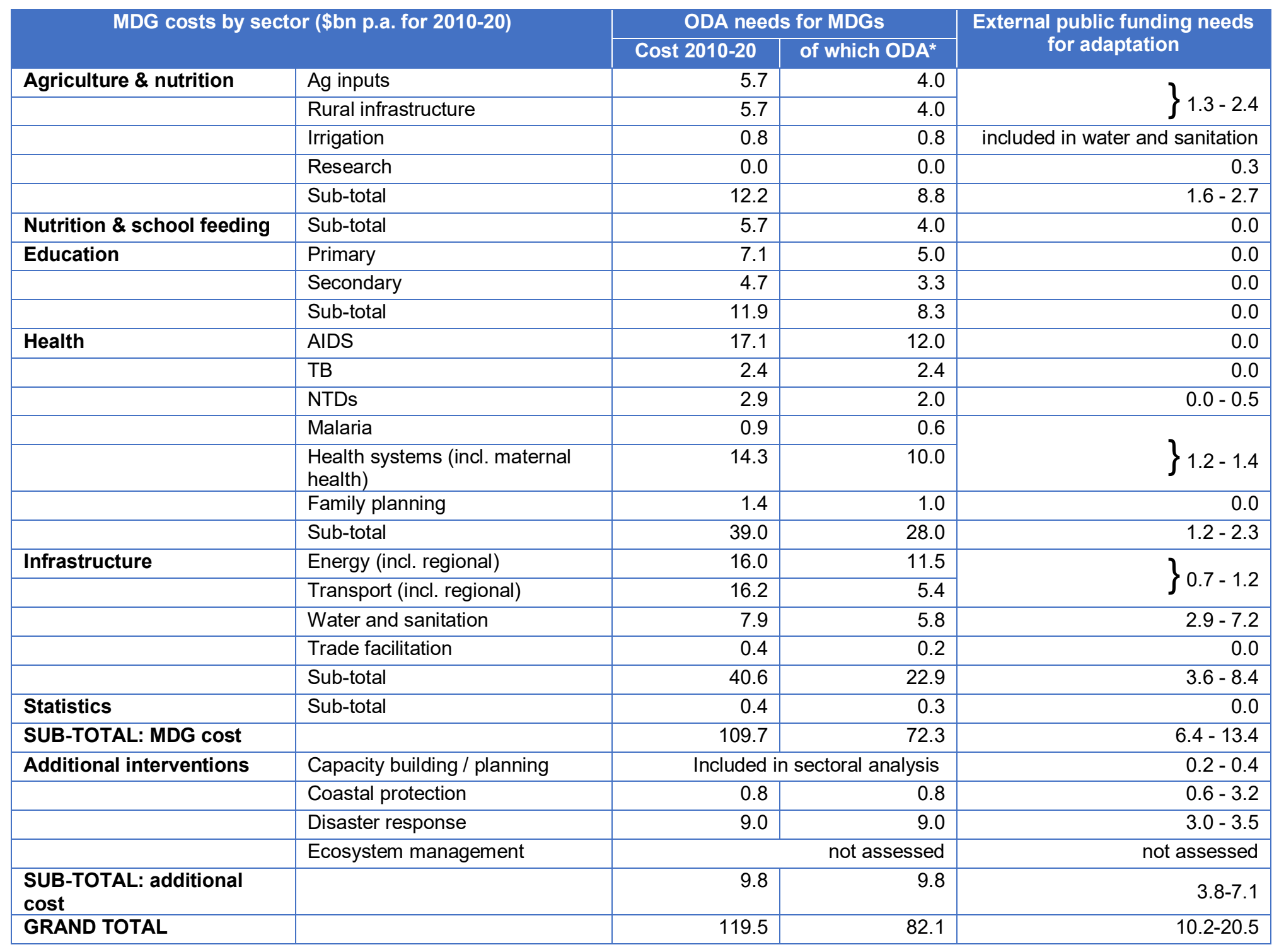


3 - The costs of climate proofing the Millennium Development Goals in Africa

Table 19 | Sources and assumptions on adaptation cost estimates

\begin{tabular}{|c|c|c|c|c|c|}
\hline \multicolumn{2}{|c|}{ Adaptation cost assumptions (\$bn pa) } & \multicolumn{2}{|r|}{ Adaptation needs $2010-20$, low } & \multicolumn{2}{|r|}{ Adaptation needs 2010-20, high } \\
\hline \multirow[t]{5}{*}{ Agriculture \& nutrition } & Ag inputs & 0.1 & $2 \%$ incremental cost (UNFCCC, 2007) & \multirow[t]{2}{*}{2.4} & \multirow[t]{2}{*}{ World Bank (2010a) } \\
\hline & Rural infrastructure & 1.1 & $\begin{array}{l}\text { 20\% mark-up for infrastructure (Stern, } \\
\text { 2007; UNFCCC, 2007) }\end{array}$ & & \\
\hline & Irrigation & 0.0 & included in water, below & 0.0 & included in water, below \\
\hline & Research & 0.3 & World Bank (2010a) & 0.3 & World Bank (2010a) \\
\hline & Sub-total & 1.6 & & 2.7 & \\
\hline $\begin{array}{l}\text { Nutrition \& school } \\
\text { feeding }\end{array}$ & Sub-total & 0.0 & $\begin{array}{l}0.33 \mathrm{~m} \text { new cases at } \$ 20 \text { each by } 2030 \text {, } \\
\text { scaled (UNFCCC, 2007) }\end{array}$ & 0.0 & $\begin{array}{l}\text { 0.33m new cases at } \$ 26 \text { each in } 2030 \text {, } \\
\text { scaled (UNFCCC, 2007) }\end{array}$ \\
\hline \multirow[t]{3}{*}{ Education } & Primary & 0.0 & \multirow[t]{3}{*}{ climate proofing buildings is minimal } & 0.0 & \multirow[t]{3}{*}{ climate proofing buildings is minimal } \\
\hline & Secondary & 0.0 & & 0.0 & \\
\hline & Sub-total & 0.0 & & 0.0 & \\
\hline \multirow[t]{7}{*}{ Health } & AIDS & 0.0 & although migration might spread AIDS & 0.0 & although migration might spread AIDS \\
\hline & TB & 0.0 & & 0.0 & \\
\hline & NTDs & 0.0 & ignored & 0.5 & use same multiplier as malaria, below \\
\hline & Malaria & 1.2 & $\begin{array}{l}17.7 m \text { new cases in } 2030 \text { at } \$ 140 \text { each; } \\
\text { scaled (UNFCCC, 2007) }\end{array}$ & 1.4 & World Bank (2010a) \\
\hline & $\begin{array}{l}\text { Health systems (incl. maternal } \\
\text { health) }\end{array}$ & & Diarrhea included in malaria above & 0.4 & $\begin{array}{l}\text { for diarrhea: } 50.3 m \text { new cases in } 2030 \text { at } \\
\$ 17 \text { each; scaled (UNFCCC, 2007) }\end{array}$ \\
\hline & Family planning & 0.0 & & 0.0 & \\
\hline & Sub-total & 1.2 & & 2.3 & \\
\hline \multirow[t]{6}{*}{ Infrastructure } & Energy (incl. regional) & 0.5 & $\begin{array}{l}20 \% \text { of ODA needs protecting at } 20 \% \\
\text { extra (Stern, 2007) }\end{array}$ & 1.2 & World Bank (2010a) \\
\hline & Transport (incl. regional) & 0.2 & $\begin{array}{l}20 \% \text { of ODA needs protecting at } 20 \% \\
\text { extra (Stern, 2007) }\end{array}$ & & included above \\
\hline & Water and sanitation & 2.9 & $\begin{array}{l}\$ 233 \text { bn over } 20 \text { years, of which } 25 \% \text { is } \\
\text { climate change (UNFCCC, 2007) }\end{array}$ & 7.2 & World Bank (2010a) \\
\hline & Africa ICT regional network & 0.0 & & 0.0 & \\
\hline & Trade facilitation & 0.0 & & 0.0 & \\
\hline & Sub-total & 3.6 & & 8.4 & \\
\hline Statistics & Sub-total & 0.0 & & 0.0 & \\
\hline \multirow[t]{5}{*}{ Additional interventions } & Capacity building / planning & 0.2 & Project Catalyst (2009), lower bound & 0.4 & Project Catalyst (2009), upper bound \\
\hline & Coastal protection & 0.6 & $\begin{array}{l}\text { Range of } \$ 528-612 m \text { for } 2030 \text { (UNFCCC, } \\
\text { 2007) }\end{array}$ & 3.2 & World Bank (2010a) \\
\hline & Disaster response & 3.0 & Webster et al. (2008) & 3.5 & Webster et al. (2008) \\
\hline & Ecosystem management & & Not assessed & & Not assessed \\
\hline & Sub-total & 3.8 & & 7.1 & \\
\hline
\end{tabular}


We use the figures prepared by the MDG Africa Steering Group as a proxy for the 'baseline' annual development expenditure during the period 2010-2020 even though the figures were prepared as point estimates for 2010. Our approach is based on two assumptions. First, we recognize that on current trajectories Africa will not meet the MDGs by 2015 . We thus interpret the MDGs as the 'maximum effort' that is practically feasible to accelerate progress in meeting basic needs in agriculture, education, health and infrastructure; and we further assume that the international commitment to make this maximum effort will be extended beyond 2015. Second, we assume that any scaling up beyond the level of maximum effort assumed in the analysis conducted by the MDG Africa Steering Group will be financed through increased domestic resources and private investment. As a result, the volume of Official Development Assistance (ODA) to Africa targeting the achievement of the MDGs in the absence of climate change is assumed constant for the period 2010-2020.

We estimate that climate-resilient development in Africa could require international financial assistance in the order of US $\$ 100$ billion per year over the period 2010-2020. This includes some US $\$ 82$ billion in 'baseline' official development assistance and US $\$ 11-21$ billion for incremental investments in adaptation. The total is about $40 \%$ higher than the original MDG estimate of US\$72 billion (Table 18).

The ODA figure reflects the fact that for the development portion substantial co-funding of about US $\$ 40$ billion would be available from national public sources. No adaptation co-funding from national sources is assumed, consistent with the provisions of the UNFCC, which offers adaptation support for least developed countries. Private investment, although central to growth and development in Africa, is not considered in this chapter since it cannot serve as a substantial substitute for the public investments required to achieve the MDGs and implement adaptation measures.

The highest MDG expenditure items are for improved health-care facilities, the fight against HIV/Aids and new energy infrastructure, which will each require annual investment in excess of US\$10 billion. The health spending would secure comprehensive primary health care (including child and maternal care), universal access to HIV treatment and the almost complete prevention of malaria deaths (see Appendix 1). Energy investment, alongside other infrastructure expenditure, would provide improved connectivity, adequate water supply and access to modern energy sources. Less costly, but equally crucial, is education and measures to combat malnutrition, such as school feeding programs. They are complemented by measures to double agricultural productivity.

The increased incidence of extreme weather events means that disaster management and social protection measures, such as access to emergency cash, move up the priority list for development spending (Annan et al., 2009). A good example of such a measure is the Productive Safety Net Program in Ethiopia, an employment-based transfer program for families affected by food insecurity (UNDP, 2007). In Table 19 these activities are recorded as 'additional development interventions', reflecting the fact that they are primarily developmental in nature even though they were not included in the assessment by the MDG Africa Steering Group.

Resource estimates for humanitarian assistance and disaster relief are difficult to obtain. In 2007, some US\$7 billion in ODA was spent on development food aid and humanitarian aid (OECD, 2009). Since the United Nations appeals for humanitarian assistance are only $51.7 \%$ funded (Webster et al., 2008), we assume that this figure represents half of actual baseline needs of some US $\$ 14$ billion globally. In recent years, some two-thirds of UN appeals for humanitarian assistance covered Africa, and so this would imply some US\$9 billion per year in baseline needs. 
As a result of climate change humanitarian costs could increase by at least $32 \%$ (Webster et al., 2008) and could rise much faster. If one accepts the lower figure as a conservative estimate for the likely humanitarian impact during the years 2010-2020, then some US $\$ 3$ billion in incremental financing will be required for climate change. 6

Among the costliest adaptation measures are water investments, which seek to preserve development achievements with respect to water access and sanitation, and investment in rural infrastructure, aimed at maintaining agricultural output. Our cost estimates for water-related infrastructure investments are derived from World Bank (2010a). Although broader in scope, they are roughly consistent with Muller (2007), who estimates adaptation costs of US\$2-5 billion a year for urban water management alone. However, our numbers probably underestimate the resources needed for climate-proofing buildings, including the upgrade of slum dwellings (Garau et al., 2005).

If investments in the MDGs and climate change adaptation are undertaken as suggested, the extra resources required for nutrition programs should be modest. Protecting education from the effects of climate change should also only require modest incremental resources, although there will be some expenditure to climate-proof school buildings. Much more noticeable will be the impact on health budgets, in particular spending on malaria protection, which will have to be extended into hitherto unaffected areas. An important area that has been omitted, both in the original MDG figures and in our extended estimate is the cost of protecting ecosystems. This is despite the fact that the preservation of ecosystem services is crucial for poverty alleviation (Chomitz, 2007; Parry et al., 2009). Future analysis will have to fill this gap.

The estimate includes a small budget for disaster preparedness (Project Catalyst, 2009) based on data from the Munich Climate Insurance Initiative. The estimate is probably at the low end, and it explicitly excludes the damage caused by extreme weather events. Adaptation estimates are concerned with the costs of reducing impact, but not the residual impacts that cannot be adapted to (Parry et al., 2009).

\subsection{Conclusions and outlook for further work}

Development and adaptation to climate change are intricately linked. In least-developed regions like parts of Africa, adaptation is to a large extent climate-resilient development or, 'development in a hostile climate' (Stern, 2009b). This chapter supports this view and advances the discussion by estimating the combined resource needs for meeting and climate-proofing the MDGs for Africa.

The starting point for our analysis is the cost estimates for achieving the MDGs prepared by the MDG Africa Steering Group. We complement them by a rough sector-bysector analysis of additional adaptation needs, using existing aggregate adaptation cost estimates from the World Bank, the UNFCCC and other sources.

We find that Africa's annual resource needs for climate-proofing the MDGs - understood as a broad outcome targets for development - is about $40 \%$ higher than the cost of meeting the MDGs alone, that is, about US $\$ 100$ billion, compared with US $\$ 72$ billion a year over the next 10 years.

Extra costs arise from having to provide more development support (e.g. extra bed nets against malaria), the same support at a higher cost (e.g. more expensive infrastructure) as well as altogether new measures (e.g. adaptive capacity building). Climate change may also require certain measures to be prioritized compared to the baseline development plan (Fankhauser and Burton, 2011). 
Treating adaptation and development in such an integrated way helps to better understand financing requirements analytically. It also provides a framework for implementing the requisite measures more effectively as part of an integrated development program. This integrated analysis might also offer a simple solution for how the 'additionality' of resources for climate change can be determined. Resources for adaptation are additional if they are provided above and beyond the promised MDG financing summarized in Table 18.

Like the original MDG estimates, our analysis is organized along sectoral lines that correspond roughly to the organizational structure of most governments, so that the numbers can be tied to explicit objectives and delivery mechanisms. We believe it to be crucial for adaptation measures to be implemented by the same ministries that are responsible for delivering development outcomes, i.e. the departments of health, education, agriculture and so on. In addition, the importance of adaptation should be recognized by the finance and economy ministries that set funding priorities, and the corresponding measures must be incorporated into a single macro-economic framework.

These estimates of resource need we provide are indicative only and imperfect in many ways. They draw heavily on existing top-down analyses of adaptation and MDG resource needs that are by necessity aggregated and subject to substantial margins of error. The reliance on existing cost data also create some inconsistencies in the time frame and other assumptions that underpin the original estimates.

Going forward, three interrelated analytical issues need to be addressed. First, remaining gaps in our analysis, such as the treatment of ecosystem management, have to be filled. Second, the integrated adaptation and development frameworks we propose need to be applied to actual development strategies at the country level, since this is where the rubber hits the road. Third, the time frame of the analysis needs to be extended, since many impacts of climate change will only materialize beyond 2020 .

\section{Acknowledgements}

This chapter has benefited greatly from comments and feedback by Philip Dobie, David Dodman, Su-Lin Garbett-Shiels, Saleemul Haq, Arnaud Henin, Dawda Jobarteh, Michael Keating, Benito Mu"ller, Achim Steiner and Nicholas Stern. Fankhauser would also like to acknowledge the financial support from the Grantham Foundation for the Protection of the Environment and the Centre for Climate Change Economics and Policy, which is funded by the UK's Economic and Social Research Council (ESRC) and by Munich Re. 


\section{Annex 3. Overview of principal MDG and adaptation investments}

\section{A3.1. Education}

Table 20 | MDG and adaptation interventions for education

\begin{tabular}{|c|c|c|c|}
\hline Development Results & Key Development Interventions & $\begin{array}{l}\text { Risks to Development } \\
\text { Results from Climate } \\
\text { Change }\end{array}$ & $\begin{array}{l}\text { Incremental Adaptation Interventions to } \\
\text { Achieve Development Results }\end{array}$ \\
\hline $\begin{array}{l}\text { - Achievement of Education for All } \\
\text { Goals by 2015: } \\
\circ \quad \text { universal primary school } \\
\text { completion; } \\
\text { comprehensive early child- } \\
\text { hood care; } \\
\text { 50\% improvement in adult } \\
\text { literacy from 2000; } \\
\text { gender equality in educa- } \\
\text { tion; } \\
\text { improved quality of educa- } \\
\text { tion; and advancing life-long } \\
\text { learning. } \\
\text { - Expanded access to secondary, } \\
\text { vocational and higher education } \\
\text { by } 2015 \text {. }\end{array}$ & $\begin{array}{l}\text { - Train, hire and retain adequate numbers of } \\
\text { teachers for primary, secondary, and voca- } \\
\text { tional schools } \\
\text { - Provide and maintain school infrastructure } \\
\text { and learning materials } \\
\text { - Remove barriers to education that depress } \\
\text { demand (e.g. school fees, lack of appropriate } \\
\text { hygiene facilities for girls, lack of transport) } \\
\text { - Provide effective schooling solutions in post- } \\
\text { conflict and humanitarian settings } \\
\text { - Design and implement locally appropriate cur- } \\
\text { ricula together with continuous monitoring of } \\
\text { learning outcomes }\end{array}$ & $\begin{array}{l}\text { - Accelerating urbaniza- } \\
\text { tion in response to fall- } \\
\text { ing agricultural yields } \\
\text { will require a faster ex- } \\
\text { pansion of urban } \\
\text { schooling opportunities } \\
\text { - Climate-induced } \\
\text { droughts and other hu- } \\
\text { manitarian disasters will } \\
\text { increase need for high- } \\
\text { cost schooling in hu- } \\
\text { manitarian settings } \\
\text { - More frequent extreme } \\
\text { weather event will in- } \\
\text { crease wear and tear on } \\
\text { school infrastructure }\end{array}$ & $\begin{array}{l}\text { - No major changes required to countries' na- } \\
\text { tional education strategies }\end{array}$ \\
\hline
\end{tabular}




\section{A3.2. Health}

\section{Table 21 | MDG and adaptation interventions for health}

\begin{tabular}{|c|c|c|c|}
\hline Development Results & Key Development Interventions & $\begin{array}{l}\text { Risks to Development } \\
\text { Results from Climate } \\
\text { Change }\end{array}$ & $\begin{array}{c}\text { Incremental Adaptation Interven- } \\
\text { tions to Achieve Development } \\
\text { Results }\end{array}$ \\
\hline $\begin{array}{l}\text { - Widespread access to comprehen- } \\
\text { sive primary health systems that } \\
\text { meet demand and supply-side con- } \\
\text { straints } \\
\text { - Universal and free access to im- } \\
\text { munization and key child survival in- } \\
\text { terventions; } \\
\text { - Universal and free access to repro- } \\
\text { ductive health services; } \\
\text { - Universal and free access to } \\
\text { HIVIAIDS prevention, mitigation } \\
\text { and treatment by } 2010 ; \\
\text { - Malaria burden halved by } 2010 \\
\text { (from } 2000 \text { levels) and malaria mor- } \\
\text { tality reduced to near zero by } 2015 \text {; } \\
\text { - Control of TB through implementa- } \\
\text { tion of Global Stop TB Plan of Ac- } \\
\text { tion; and } \\
\text { - Sharply reduced morbidity and mor- } \\
\text { tality from Neglected Tropical Dis- } \\
\text { eases (NTDs) and other diseases } \\
\text { prevalent in the country. }\end{array}$ & $\begin{array}{l}\text { - Establish and maintain effective primary health sys- } \\
\text { tems, including the provision of } \\
\circ \quad \text { Adequate human resources for the management } \\
\text { and provision of health services at all levels, in- } \\
\text { cluding community health workers; } \\
\text { Adequate access to essential drugs and commod- } \\
\text { ities; } \\
\text { Adequate supply and logistics systems; and } \\
\text { - Inter alia, the health systems should provide the follow- } \\
\text { ing key interventions: } \\
\text { Immunization, neonatal integrated package, inte- } \\
\text { grated management of childhood illnesses } \\
\text { Micronutrient and Vitamin A supplementation } \\
\text { Full range of reproductive health services, includ- } \\
\text { ing emergency obstetrical care, antenatal care, } \\
\text { skilled birth attendants and family planning } \\
\text { Universal and free access to HIVIAIDS ARV treat- } \\
\text { ment, voluntary counseling and testing, preven- } \\
\text { tion of mother-to-child transmission, other mitiga- } \\
\text { tion and prevention measures } \\
\text { Directly-Observed Short Treatment (DOTS) and } \\
\text { other interventions identified in Global Stop TB } \\
\text { Plan of Action } \\
\text { Universal access to long-lasting insecticide- } \\
\text { treated bed nets, effective anti-malarial drugs (e.g. } \\
\text { ACT), and, where necessary, residual indoor } \\
\text { spraying. } \\
\text { Treatment and prevention of NTDs }\end{array}$ & $\begin{array}{l}\text { - Rising temperatures } \\
\text { may facilitate propaga- } \\
\text { tion of pathogens and } \\
\text { promote diarrhea. } \\
\text { - Increased migration in } \\
\text { response to climate } \\
\text { change may accelerate } \\
\text { spread of TB, sexually } \\
\text { transmitted infections } \\
\text { and other infectious dis- } \\
\text { eases. } \\
\text { - Increased incidence and } \\
\text { prevalence of vector- } \\
\text { borne diseases (e.g. ma- } \\
\text { laria, NTDs) increase } \\
\text { disease burden and un- } \\
\text { dermine economic de- } \\
\text { velopment. }\end{array}$ & $\begin{array}{l}\text { - Increased investments in pre- } \\
\text { vention and treatment of sex- } \\
\text { ually-transmitted infections, TB, } \\
\text { and other infectious diseases. } \\
\text { - Provision of long-lasting insecti- } \\
\text { cide-treated bed nets to popula- } \\
\text { tions who are newly exposed to } \\
\text { malaria, expanded residual in- } \\
\text { door spraying, and supply of ef- } \\
\text { fective anti malarials. } \\
\text { - Expand emergency health sys- } \\
\text { tems in response to a projected } \\
\text { increase in the incidence of epi- } \\
\text { demic disease outbreaks and } \\
\text { other humanitarian challenges. }\end{array}$ \\
\hline
\end{tabular}




\section{A3.3. Agriculture, food security, and nutrition}

Table 22 | MDG and adaptation interventions for agriculture, food security, nutrition

- Sustainable doubling of food yields across Africa to reduce poverty, hunger and malnutrition

- Subsistence agriculture progressively transformed into commercial agriculture to accelerate economic growth

- Soil health and prevention of desertification

- Improved child nutrition and learning outcomes through national school feeding programs and other nutrition programs

- Adequate provision of micronutrients to populations at risk, including children aged $0-2$ years, combined with effective de-worming to ensure nutrient absorption.
- Launch an African Green Revolution within the framework of Comprehensive Africa Agriculture Development Program (CAADP). Key interventions include providing access to improved seeds, fertilizers and agricultural as well as financial extension services; strengthening land and water management; improving rural infrastructure; strengthening farmers' associations; and increasing access to markets in close collaboration with the private sector.

- Support these interventions by reforms of agricultural policies and institutions as well as local purchases of food assistance.

- Implementation of soil erosion control (by wind and water) by planting windbreaks and cover crops; improvements in soil fertility with agroforestry systems, cover crops, and conservation of ground and surface water.

- Roll out school feeding programs using locally produced food that cover all children in primary school.

- Establish comprehensive national-scale nutrition programs to tackle micronutrient deficiencies (i.e., lodine, Vitamin A, Zinc, Iron, etc.) with a particular focus on children aged $0-2$. Providing take-home food rations will increase incentives for girls to attend schools.

- Scale up investment in agricultural research into highyielding crop and livestock varieties as well as sustainable agricultural practices. Incremental investments need to adhere to the CAADP in particular its Framework for African Agricultural Productivity (FAAP), and support African research through the Forum for Agricultural Research in Africa (FARA), sub-regional organizations, and centers belonging to the Consultative Group on International Agricultural Research (CGIAR).
Risks to Development Results from Climate Change

- Falling agricultural yields and increasing climate variability will depress farmers' incomes and increase their economic vulnerability to weather-related crop failures.

- Increased frequency and severity of weather-induced crop failures (in particular farmers will be vulnerable to the premature failure of rains towards the tail end of the growing season when the impact on crops will be greatest)

- Increased frequency and severity of droughts that threaten livestock and pastoralists' assets.

Rising temperatures may prop agate pests and animal diseases.

- Increased competition over scarce water resources among farmers and pastoralists.

- The incidence of famines and malnutrition may rise.
Incremental Adaptation Interventions to Achieve Development Results

- As part of integrated water resource management strategies, invest in the software and hardware of collecting, storing, distributing and using water for agricultural purposes. In particular, increased water storage will be required, much of it in the form of smallscale infrastructure constructed by farmers that can ensure a successful harvest if rains fail towards the end of the growing season.

- Expand irrigation systems and increase efficiency through development of efficient irrigation systems, including drip irrigation.

- Expand soil erosion control programs, including the planting of windbreaks and cover crops; improvements in soil fertility with agroforestry systems, cover crops, and conservation of ground and surface water.

- Increase expenditure on agricultural research to promote the development of drought-resistant crops as well as germplasm that can withstand higher temperatures.

- Expand public programs for the provision of key agricultural inputs, such as fertilizer and improved seeds that can increase farming yields and strengthen the economic resilience of communities.

- Strengthen agricultural extension to support the shift towards farming practices that are better aligned with a changing climate.

- Expand pest monitoring and control programs, including comprehensive vaccination of livestock

- Expand school feeding and other nutrition programs (e.g. targeting pregnant mothers and young infants).

- Increase budgets for emergency feeding programs in response to disasters 


\section{A3.4. Infrastructure and trade facilitation}

\section{Table 23 | MDG and adaptation interventions for infrastructure, trade facilitation}

Development Results

- Adequate connectivity and infrastructure to increase productivity, ensure low-cost service delivery, and integrate African countries into the global economy through:

- Effective regional networks for roads, rail, canals, power pools, and information and communications technology to integrate African economies and to provide landlocked countries with reliable access to seaports;

- Adequate rural and urban electrification and access to other modern energy services;

- Adequate transport grids, including major expansion of rura feeder roads;

- By 2015, halve the proportion of people without access to adequate water supply and sanitation; and

- Strengthen national and regional institutions to promote regional integration, regional infrastructure projects and trade facilitation across Africa.
Key Development Interventions

- Plan and build transformational generation and transmission facilities across Africa, and improve the performance of power utilities.

- Develop decentralized energy systems to increase access to fuels for domestic cooking and heating, motive power and off-grid electricity.

- Expand the construction and maintenance of all-weather roads, including urban road networks

- Provide adequate urban infrastructure (slum upgrading, transport, energy, water drainage, sewage, lighting)

- Implement national strategies to achieve the water supply and sanitation MDG targets.

- Develop regional infrastructure (e.g. road corridors, power pools, multipurpose water infrastructure, information and communications technology), as outlined in the African Union NEPAD Infrastructure Short-Term Action Plan and other regional plans.

- Implement the Enhanced Integrated Framework and Aid for Trade to support country efforts to develop their trade capacity and performance.
Risks to Development Reults from Climate Change

- Increased intra- and interannual variability in precipitation will increase need for water storage for agriculture and domestic water use.

- Hydropower generation capacity of existing infrastructure may fall and suffer from increased intermittency.

- Rising incidence of extreme precipitation and other weather events will increase wear and tear of ransport infrastructure particularly roads.

- Increased flooding in urban areas.

- Vulnerability to sea level rise and salt water intrusion into aquifers.
Incremental Adaptation Interventions to Achieve Development Results

- Expand construction of water storage capacity for power generation and retention of run-off for agricultural and domestic use.

- Increase access to deep boreholes and water wells to provide year-round access to clean drinking water.

- Expand regional power pools to use available hydropower resources more effectively.

- Upgrade existing roads to reduce vulnerability to extreme precipitation event; expand road maintenance operations.

- Expand storm water drainage.

- Build sea walls, expand flood management systems, and control aquifer discharge to minimize salt water intrusion. 


\section{A3.5. Other development needs}

\section{Table 24 | MDG and adaptation interventions for other development needs}

Table summarizing development needs not included in recommendations by Africa Steering Group (Ki-Moon et al., 2008)

\section{Development Results}

- Effective emergency sponse systems

re-

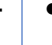

- Environmental sustainability objectives not covered above

Sustainable forest management

- Sustainable use and management of watersheds and wetlands
National and regional monitoring and earlywarning systems for emergency and humanitarian responses.

\section{Key Development Interventions}

Implementation of sustainable forest management techniques, forest plantations in appropriate areas to satisfy demand for forestry products, and tree seedlings and other measures to support afforestation.

- Institution of Integrated Water Resources Management plans; promotion of reforestation to protect selected catchment areas; and monitoring of wells and groundwater-dependent systems.
Risks to Development Results from Climate Change

- Increased likelihood and severity of emergencies and humanitarian disasters

- Forests will come under increasing pressure from raising temperatures, and desertification will accelerate in parts of Africa.

- The hydrological cycle will undergo profound changes in response to climate change, thus undermining IWRM efforts in many parts of the continent.
Incremental Adaptation Interventions to Achieve Development Results

- Strengthen emergency and humanitarian systems

- Interventions to protect forests need to be adapted to rising temperatures and possible changes in locally appropriate tree species. (Large-scale reforestation and avoided deforestation measures fall under 'mitigation' and are not considered in this chapter.)

- Weather and climate monitoring stations need to be installed and maintained across Africa to provide reliable, realtime meteorological information.

- Monitoring of groundwater aquifers will need to be scaled up. (Other IWRM interventions are considered above) 


\section{On metrics and financing for the Sustainable Development Goals}

This chapter is a consolidated version of the working paper: Schmidt-Traub, G. 2015. Investment Needs to Achieve the Sustainable Development Goals in Low- and Lower-Middle Income Countries. SDSN Working Paper. SDSN: Paris and New York. 


\section{Chapter 4}

\section{Investment needs to achieve the}

Sustainable Development Goals in low- and lower-middleincome countries

\begin{tabular}{|c|c|c|c|c|c|}
\hline Whan & 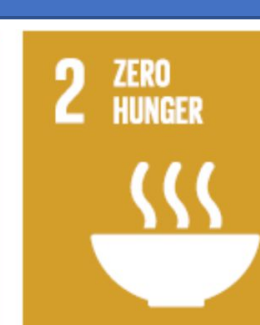 & 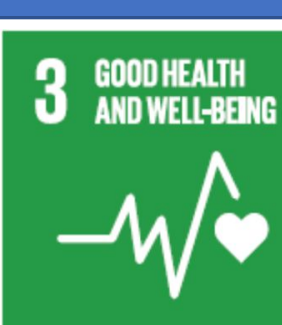 & 4 inthenture & 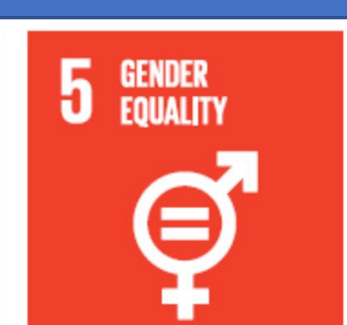 & 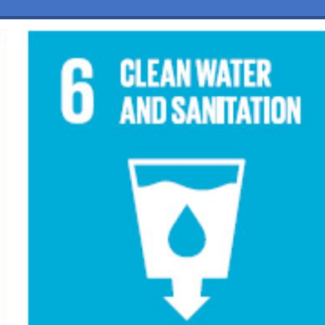 \\
\hline 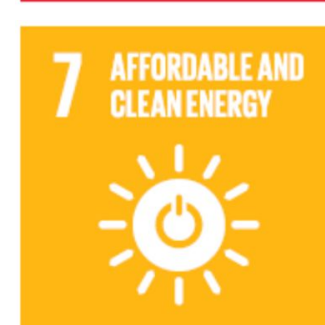 & 1 & $\mathrm{sta}$ & e & & \\
\hline 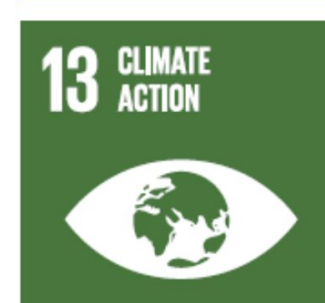 & & 5 㟧 & 16 16 & & \\
\hline
\end{tabular}


On metrics and financing for the Sustainable Development Goals

In September 2015, member states of the United Nations adopted the Sustainable Development Goals (SDGs) to guide international cooperation in pursuit of ambitious quantitative goals to be achieved by 2030. This chapter assesses how sector needs assessments can help identify and program the investments needed to achieve the goals. It proposes an analytical framework for SDG needs assessments that translates the 17 SDGs into eight investment areas and introduces a preliminary score to assess the quality and suitability of needs assessment studies. Using this framework, published sector needs assessments are analyzed, harmonized, and consolidated. A first assessment of private and public investment needs for the SDGs is provided, and implications for financing the SDGs are explored. This study finds that incremental spending needs in low- and lower-middle-income countries amount to at least \$1.3-1.5 trillion per year. Approximately half of these incremental investments can be privately financed. Domestic resource mobilization can increase significantly, leaving an external financing gap of perhaps \$152-163 billion per year (equivalent to $0.22-0.26 \%$ of highincome countries' GDP) that must be met through international public finance, including Official Development Assistance. Globally, an incremental 1.5-2.5\% of world GDP needs to be invested each year by the public and private sectors to achieve the SDGs in every country. By providing the first consistent framework and estimates of SDG investment needs, the chapter informs policy for SDG implementation and highlights research gaps that need to be filled to improve our understanding of how the SDGs can be financed. 


\subsection{Introduction and purpose}

Member states of the United Nations adopted the Sustainable Development Goals (SDGs) on 25 September 2015 (United Nations, 2015a). The World Bank (Kim, 2015) and IMF (2015b) have committed to support the implementation of the 17 goals, which map out ambitious objectives across the three dimensions of sustainable development (economic development, social inclusion, environmental sustainability) to be achieved by 2030 . These SDGs provide quantitative milestones for sustainable development, which extend the Brundtland et al. (1987) definition of sustainable development as "development that meets the needs of the present generation without compromising the ability of future generations to meet their own needs." On current trends these goals will not be met (Joshi et al., 2015).

As shared goals of governments, international financial institutions, and other stakeholders, the SDGs raise the question how they can be translated into budgets, medium-term expenditure frameworks, and macroeconomic programming, which in turn requires a detailed understanding of required public and private investment needs. This chapter contributes to the conceptualization of these public and private investments by addressing two sets of issues. First, it presents an analytical framework that allows researchers to compare needs assessments or 'costings'; assess their completeness, robustness, and usefulness for guiding the programming of investments; and determine how to aggregate them. The chapter proposes a preliminary suitability score for technical analyses of sector investment needs. Second, it applies this analytical framework in a manner that is incomplete to determine incremental public and private investment needs to achieve the SDGs in low- and lower-middle-income countries and globally. The chapter reviews published studies, many of which lack the rigor of academic research and require improvement through peer-review. Given these limitations, this chapter cannot vouch for the results obtained by the studies it reviews. The analysis does suggest that financing needs for the SDGs are in the range of $2 \%$ of world GDP and unlikely to exceed $2.5 \%$. The chapter aims for maximum transparency in exposition and analysis (see Annex), so that researchers can draw their own conclusions and identify the most promising avenues for research. Its findings point to research questions that require careful study to determine how investments in the SDGs might be structured and financed, and how supporting analytical frameworks can be improved.

One recent strand of applied welfare analysis has tackled sustainable development by focusing on estimating adjusted or genuine savings rates (Pearce and Atkinson, 1993; Hamilton and Clemens, 1999; World Bank, 2011). This literature asks whether national saving is high enough to ensure rising living standards, comprehensively measured to include dimensions beyond income. Specifically, the 'genuine saving rate' adjusts the definition of the saving rates in national accounts by including human capital, natural capital, and the depletion of natural resources, such as oil, gas, or timber. Arrow et al. (2012) introduce the closely related concept of inclusive wealth (IW), which describes the value of future wellbeing $(\mathrm{V})$, defined as the integral of discounted future true consumption. IW is a function of various forms of capital (human capital, natural capital, health capital, and reproducible (business) capital) weighted by their respective shadow prices. Genuine saving may then be equated with the rise in IW. Countries with a decline in IW are dis-saving, typically because some of what is currently counted as national income is in fact the depletion of natural capital.

The Brundtland definition of sustainable development requires that $\mathrm{dV}>0$, that is a rise in the discounted future value of true consumption. In turn, $d V>0$ requires positive 'genuine saving' to raise inclusive wealth. IW can be calculated, at least in part, to see whether a society is achieving sustainable development in the Brundtland sense, 
though the data challenges to this exercise are substantial (Arrow et al., 2012; UNUIHDP and UNEP, 2016).

Instead of asking whether $\mathrm{dV}>0$ (c.f. the Brundtland test), this chapter asks, albeit in a manner that is still highly incomplete, how the composition and scale of investment profiles need to change for an economy to achieve the SDGs by 2030. This is a more stringent test than $\mathrm{dV}>0$, particularly for low-income countries where the inclusive wealth will need to rise quickly to provide the income, social services, infrastructure, and environmental management to achieve SDG standards of wellbeing by the target date of 2030. In some sectors, such as health and education, the composition of current investments will need to change little but the scale will need to increase substantially, so the focus is on increasing the volume of investments. In other areas, such as the decarbonization of the energy system, the composition of investments must also change significantly alongside an increment of investment flows.

A sector needs assessment projects the scale and composition of public and private investment needs for a country or a set of countries to achieve specific development outcomes, such as the SDGs. Here, the term 'investments' includes capital and operating expenditure needed to achieve the outcomes laid out in the SDGs. Methodologies for needs assessments - sometimes referred to as costings - were pioneered in the early 2000s for the health sector $(\mathrm{CMH}, 2001)$, where they have since improved significantly (Waage et al., 2010; Jamison et al., 2013). Over time, they have been developed for most investment areas covered by the Millennium Development Goals (MDGs), a set of eight global development goals derived from the Millennium Declaration adopted in 2000 (UN Millennium Project, 2005; Commission for Africa, 2005; KiMoon et al., 2008; Bourguignon et al., 2008). Today, each investment area covered by the SDGs has one or more needs assessments.

SDG needs assessments can play several roles in supporting analysis and policies in support of the SDGs. First, they offer a methodology for understanding the investments required to achieve the SDGs and for identifying knowledge gaps in the understanding of implementation strategies or 'production functions' for each goal. Needs assessments reviewed in this chapter suggest that not all SDG sectors have developed a clear understanding of the types and scale of required investments. Many sectors cannot adequately model the change in composition of investments needed to achieve the SDGs. Second, needs assessment results provide a basis for determining how the SDGs can be financed through a combination of private investments and domestic public finance, taking into account government's overall budget constraint as well as concessional and non-concessional international public finance. Third, the scale and nature of projected investment needs obtained through needs assessments can help to design medium-term expenditure frameworks and macroeconomic management strategies to accommodate large changes in the scale and composition of public and private spending, as illustrated by the IMF for the MDGs in Africa (IMF, 2008; Mongardini and Samake, 2009). Fourth, needs assessments can support resource mobilization and provide an accountability framework for implementation, as illustrated by successful replenishment rounds of the International Development Association (IDA, 2013), the Global Fund (GFATM, 2015a), Gavi (2014), or the Global Financing Facility (GFF, 2015). Each of these financing mechanisms conducted needs assessments to determine the volume of required resources, identify results that would be achieved with greater financing, and propose an accountability framework to track the effective use of additional resources.

Needs assessments for global goals are imperfect analytical tools and therefore controversial. Some argue that policies and good governance are more important than investment needs, so focusing on investment needs is misguided (ODI et al., 2015; 
Devarajan et al., 2002). Yet, evidence reviewed in this chapter suggests that sound policies as well as changes in investments are needed to achieve the SDGs. Needs assessments can address policy issues, such as removing user fees for health services (WHO, 2010; Jamison et al., 2013; Chatham House, 2014), subsidies for cooking fuels (Pachauri et al., 2013), or providing feed-in tariffs for renewable energy (NCEC, 2014).

A second critique is that needs assessments do not adequately consider absorptive capacity constraints (Clemens et al., 2007), defined as the ability to scale up public investments efficiently at the sector and macroeconomic level. At the sector level, needs assessments help identify capacity constraints (e.g. human resources, management and monitoring systems, infrastructure) and outline ways in which they can be addressed. At the macroeconomic level, foreign currency inflows may lead to real exchange rate appreciations and shift domestic investments away from the tradable sector. As shown by the IMF, detailed needs assessments are a prerequisite to understanding how countries need to adjust their macroeconomic frameworks and policies to mitigate adverse consequences from foreign currency inflows (IMF, 2008; Mongardini and Samake, 2009; Prati et al., 2003).

Third, some observers argue that results from needs assessments are too unreliable to be useful for policy purposes (ODI et al., 2015; Reddy and Heuty, 2006) and that unit costs cannot be known beyond marginal increments (Bourguignon et al., 2008). Technologies evolve in ways that are impossible to predict with certainty over a 15year period, and long-term price developments are uncertain. These are fundamental challenges that caution against taking SDG needs assessments too literally and reinforce the need to consider them in conjunction with dynamic economy-wide tools (see next section). On the other hand, policymakers trying to implement the SDGs are faced with complex questions, namely whether it is technically possible to achieve ambitious long-term goals, whether overall investment needs can be met through savings, and how to structure medium-term expenditure frameworks that are consistent with such goals. These require a longer-term understanding of the necessary infrastructure, human resources, and other investment needs, which can only be provided through transparent needs assessments. In the case of the energy transition, Williams et al. (2012, 2014) and SDSN and IDDRI (2015) demonstrate how long-term technology pathways are required through to 2050 and beyond in order to design short-term public and private investment decisions consistent with respecting a global carbon budget and to help guide policies and public-private investments in support of low-carbon technology development. Similarly, long-term health needs assessments have helped guide investments in new health technologies under the MDGs (Jamison et al., 2013).

This chapter show that the range of investment estimates can indeed be large across available studies. Many needs assessments lack transparency and have yet to be subjected to the rigor of academic peer review. One purpose of this chapter is therefore to propose an analytical framework for transparent SDG needs assessments that will help determine appropriate methodologies, harmonize the underlying data, foster greater peer review, and thereby develop a shared understanding of how to project sector investment needs, however imperfectly.

Finally, Easterly $(2005,2006)$ and others argue that needs assessments do not focus enough on economic growth and that by focusing on supply they neglect the demand side, which is equally important. Economic growth is vital to achieve the SDGs, but as demonstrated by the health sector $(\mathrm{CMH}, 2001)$, some goals cannot be achieved through economic growth alone and require targeted public and private investments. However, the needs assessments reviewed in this chapter do not model the rise in 
private business capital investment which, together with spending on health, education, infrastructure, and environmental management, would be sufficient to achieve the target rates of economic growth implied by the SDGs. This type of modeling - combining sector investments with aggregate growth dynamics, including private business investment and the demand side - will require complementary economic tools that are reviewed briefly in the next section. Their full application is, however, beyond the scope of this chapter.

On balance, needs assessments are an imperfect tool to address complex, long-term questions around SDG implementation. They should be used in conjunction with other analytical tools, such as economy-wide models, and they will require ongoing refinement and revisions. The current practice of conducting long-term needs assessment leaves significant room for improvement, which this chapter aims to help chart out by proceeding as follows: Section 4.2 describes available data sources and reviews available needs assessment methodologies. It proposes an analytical framework for estimating total SDG investment needs by aggregating sector investment needs, taking account of overlaps, synergies, and trade-offs. The methodology pays attention to cross-cutting investments needs in climate change and other areas. Section 4.3 presents public and private investment needs by SDG investment area, aggregates the results for low- and lower-middle-income countries (LICs and LMICs), extrapolates global investment needs for the SDGs, and indicates how a financing strategy might be developed. Section 4 discusses policy implications and highlights major areas that require additional research.

\subsection{Method and data sources}

The SDGs describe broad outcome objectives that require many inputs. In a series of many-to-many relationships, achieving each SDG requires many inputs, and each input may contribute to more than one SDG. For this reason, an SDG needs assessment cannot be arranged by outcome goals since this would lead to double-counting of investment needs for inputs that contribute to more than one goal. To develop an analytical framework for SDG needs assessment, the chapter draws on UN Millennium Project (2005) analysis for MDGs. It divides the inputs required for the SDGs into eight SDG investment areas (excluding areas that can be entirely financed through private commercial investments, such as industrial development): (1) Health; (2) Education; (3) Social protection; (4) Food security and sustainable agriculture; (5) Infrastructure, including (5.1) Energy access and low-carbon energy infrastructure; (5.2) Water and sanitation; (5.3) Transport infrastructure; (5.4) Telecommunications infrastructure; (6) Ecosystem services and biodiversity; (7) Data for the SDGs; and (8) Emergency response and humanitarian work.

Cross-cutting issues can be addressed across these eight SDG investment areas: poverty, climate change mitigation and adaptation, gender equality, reducing inequalities, cities and human settlements, sustainable consumption and production, government functions, operating the international system, security and peacekeeping. In addition, this chapter follows the analytical framework developed in Chapter 3 to include incremental investment needs for climate change adaptation and mitigation in each major SDG investment area. The principal sources for climate-related investments are World Bank (World Bank, 2010c) and the Economics of Adaptation project (ECONADAPT, 2015).

Available needs assessments differ in methodologies, coverage, assumptions, and robustness, which makes them difficult to compare (UN Millennium Project, 2004; UNTT, 
2013). The methodology of choice for estimating SDG investment needs are intervention-based needs assessments as employed by UN Millennium Project (2005) and most recent assessments including in health (Chatham House, 2014; Jamison et al., 2013), education (UNESCO, 2015a), water and sanitation (Hutton, 2015), data (Espey et al., 2015), and ecosystem services (CBD, 2012a, 2012b). This approach consists of specifying each intervention, defined as the provision of goods, infrastructure, or services, needed to achieve the desired outcomes. Tools are then used to estimate the capital and operating expenditure required to deliver theses interventions to the target populations. Unit costs may change with increasing coverage (e.g. as populations become harder to reach), and the ratio of capital to operating expenditure will evolve over time as countries expand their capital stock. As a result, marginal investment needs for expanding social services and providing access to infrastructure services change over time. Since intervention-based tools are often designed in the form of spreadsheets, they are comparatively transparent, easy to use, and can serve as an accountability framework because they track outputs and outcomes to inputs. A downside of this approach is the lack of dynamic cross-sectoral assessments of synergies and trade-offs, which require exogenous adjustments. Similarly, intervention-based tools cannot consider economy-wide effects dynamically.

Four other methodologies are frequently used (UN Millennium Project, 2004; UNTT, 2013):

- Simple unit cost estimates: This approach is a variant of intervention-based needs assessments but uses more aggregate unit costs, such as the cost of schooling per pupil (Delamonica et al., 2001) or the cost of maintaining protected areas per square kilometer (CBD, 2012a, 2012b). These methodologies are simple to use but lack the level of detail to support the programming of public expenditure. They also tend to be based on historic unit costs that may not apply as more marginal populations need to be reached.

- Incremental Capital-Output Ratio (ICOR) and outcome elasticities: This methodology estimates incremental investment needs to generate a target investment rate for reducing extreme poverty (Devarajan et al., 2002) or for meeting sector targets (FAO et al., 2015b). Some econometric models (Bhattacharya et al., 2012; Fay et al., 2011; Foster and Briceño-Garmendia, 2010) similarly regress infrastructure investments on economic growth. In all cases, the results are based on historic growth elasticities that are difficult to estimate, cannot anticipate investment needs that respond to new challenges, and do not map out investment needs at the level of detail and specificity that policymakers need to program public expenditure and develop accountability frameworks. Moreover, they are not goal-based as they do not work backward from target coverage rates, as required under the SDGs. ICORs or other aggregate outcome elasticities are therefore not suitable for estimating SDG investments needs.

- Computable General Equilibrium (CGE) models: CGEs consist of aggregate production and utility functions that are combined to model an economy in equilibrium. Changes can then be introduced into the system to estimate the investment needs for different policy options. Agénor et al. (2005) first applied a simple CGE model to the MDGs. The World Bank's more sophisticated Maquette for MDG Simulations (MAMS) (Lofgren and Diaz-Bonilla, 2008; Lofgren et al., 2013) models subsets of some MDG sectors (primary education, health, water and sanitation) and combines them with a generic economic model, allowing the tool to address interactions across sectors and economy-wide effects, such 
as economic growth, real wages, real exchange rates, as well as public and private investment, saving, and consumption (Bourguignon et al., 2008). Yet, the parametrized Cobb-Douglas production functions used for development outcomes in education, health, and other sectors are too stylized to model the investment needs in individual sectors or to guide budgetary processes. Moreover, the computational complexity and data requirements limit the models' scope to a relatively small subset of SDG investment areas. As stated by Bourguignon, Diaz-Bonilla, and Lofgren (2008): "MAMS does not replace detailed sector studies, but instead complements [them]". Sector needs assessments and economy-wide models address different but complementary questions.

- Integrated Assessment Models (IAMs): Such models incorporate information from a range of disciplines in a consistent and dynamic manner, typically with a focus on biophysical systems, such as the climate. IAMs tend to model economic systems in less detail than CGEs. Prominent examples for IAMs are DICE (Nordhaus, 2008), IMAGE (PBL, 2015), and MESSAGE (Messner and Strubegger, 1995). Most IAMs are not primarily designed for estimating investment needs and therefore do not produce budgets that can be tied directly to inputs, outputs, and longer-term outcomes. They also tend to be difficult to interpret, even for experienced experts, since assumptions can be deeply embedded in the models (UNTT, 2013).

This chapter analyzes published sector needs assessments at the global level or for groups of countries across the eight SDG investment areas, introduces a preliminary score to assess and compare the quality and suitability of the underlying analysis ('suitability score'), and identifies adjustments to be made to available needs assessments (Table 25 and Annex). The new and preliminary suitability score assesses available needs assessments against nine questions that must be addressed to inform the programming of public and private expenditure for the SDGs: (1) Is the needs assessment intervention-based? (2) Are inputs clearly identified to address gaps and overlaps with other needs assessments? (3) Are interventions required to achieve each outcome goal addressed comprehensively? (4) Is the analysis goal-based? (5) Has the assessment been peer reviewed? (6) Are both operating and capital expenditure included? (7) Can results be disaggregated by LICs and LMICs? (8); Are investment needs for climate change adaptation and mitigation considered (if applicable)? (9) Have results from dynamic economy-wide models been considered in the sector assessment? Each criterion is assigned a binary score of one or zero, and the results are added up and scaled from 0 to 10. Details on data and methodology are provided in the Annex. An online supplementary data file provides full details on the calculations: http://unsdsn.org/resources/publications/sdg-investment-needs/.

The chapter then estimates incremental investment needs for each SDG investment area. Investments denote all operating and capital expenditure that are needed to achieve the SDGs. Total SDG investment needs cannot be estimated at this stage since available data on current government and private expenditure are too sparse. For this reason, the analysis focuses on incremental investments above the level of current public and private expenditure for the base year 2013.

This approach, required by the availability of data, makes it impossible to quantify changes in the composition and volume of current expenditure devoted to the SDGs, particularly in response to climate change mitigation and adaptation. Following the available literature, the chapter applies climate change mark-ups to incremental sector investment needs derived from studies that do not consider climate change adaptation or mitigation. Since data on the composition and volume of current public and private 
Table 25 | Summary of sector needs assessments and adjustments made

Table summarizes sources, methodologies, suitability score, and major adjustments made to the original analyses. Minor adjustments, such as rebasing to $\$ 2013$, are described in Annex. Source: Author's analysis.

\begin{tabular}{|c|c|c|c|c|}
\hline $\begin{array}{l}\text { Investment } \\
\text { Area }\end{array}$ & Study & $\begin{array}{c}\text { Type of } \\
\text { methodology }\end{array}$ & $\begin{array}{l}\text { Suitability } \\
\text { score (out } \\
\text { of 10) }\end{array}$ & Major adjustments made \\
\hline Health & $\begin{array}{l}\text { Jamison et } \\
\text { al. (2013) }\end{array}$ & $\begin{array}{l}\text { Intervention- } \\
\text { based needs } \\
\text { assessment }\end{array}$ & 8.8 & $\begin{array}{l}\text { Augmented with estimates for non-communicable } \\
\text { diseases (WHO, 2011) and for adaptation to } \\
\text { climate change (Pandey, 2010). }\end{array}$ \\
\hline Health & $\begin{array}{l}\text { WHO } \\
(2011)\end{array}$ & $\begin{array}{l}\text { Intervention- } \\
\text { based needs } \\
\text { assessment }\end{array}$ & 6.3 & No major adjustments. \\
\hline Education & $\begin{array}{l}\text { UNESCO } \\
(2015 a \text {, } \\
2015 b)\end{array}$ & $\begin{array}{l}\text { Intervention- } \\
\text { based needs } \\
\text { assessment }\end{array}$ & 7.5 & No major adjustments. \\
\hline $\begin{array}{l}\text { Food security \& } \\
\text { agriculture }\end{array}$ & $\begin{array}{l}\text { FAO et al. } \\
(2015 b)\end{array}$ & $\begin{array}{l}\text { Incremental } \\
\text { Capital-Output } \\
\text { Ratio (ICOR) } \\
\text { estimate }\end{array}$ & 4.4 & $\begin{array}{l}\text { To avoid overlaps with other SDG investment } \\
\text { areas, removed investment needs for social } \\
\text { protection, rural roads and rural electrification. } \\
\text { Augmented with incremental investment needs for } \\
\text { climate change adaptation and mitigation } \\
\text { (UNFCCC, 2007). }\end{array}$ \\
\hline $\begin{array}{l}\text { Energy access } \\
\& \text { low-carbon } \\
\text { power } \\
\text { infrastructure }\end{array}$ & $\begin{array}{l}\text { Pachauri et } \\
\text { al. (2013) }\end{array}$ & $\begin{array}{l}\text { Integrated } \\
\text { Assessment } \\
\text { Modeling }\end{array}$ & 7.8 & $\begin{array}{l}\text { Augmented with estimates for power generation, } \\
\text { transmission and distribution (World Bank, 2013) } \\
\text { (see below for adjustments to that study) and a } \\
\text { mark-up on capital expenditure for climate } \\
\text { adaptation (Stern, 2007). }\end{array}$ \\
\hline $\begin{array}{l}\text { Energy access } \\
\text { \& low-carbon } \\
\text { power } \\
\text { infrastructure }\end{array}$ & $\begin{array}{l}\text { World Bank } \\
(2013)\end{array}$ & $\begin{array}{l}\text { Simple unit cost } \\
\text { estimates }\end{array}$ & 2.2 & $\begin{array}{l}\text { Adjusted to include operating expenditure (Foster } \\
\text { and Briceño-Garmendia, 2010). Mark-ups on all } \\
\text { costs for climate mitigation (NCEC, 2014) and } \\
\text { capital expenditure adaptation (Stern, 2007). } \\
\text { Estimates for access to rural electrification } \\
\text { (Pachauri et al., 2013) removed to avoid overlaps. }\end{array}$ \\
\hline $\begin{array}{l}\text { Water and } \\
\text { sanitation }\end{array}$ & $\begin{array}{l}\text { Hutton } \\
(2015)\end{array}$ & $\begin{array}{l}\text { Intervention- } \\
\text { based needs } \\
\text { assessment }\end{array}$ & 6.7 & $\begin{array}{l}\text { Augmented with estimates for water and sanitation } \\
\text { infrastructure (World Bank, 2013) and mark-up on } \\
\text { capital expenditure for climate mitigation } \\
\text { (UNFCCC, 2007). }\end{array}$ \\
\hline $\begin{array}{l}\text { Water and } \\
\text { sanitation }\end{array}$ & $\begin{array}{l}\text { World Bank } \\
\text { (2013) }\end{array}$ & $\begin{array}{l}\text { Simple unit cost } \\
\text { estimates }\end{array}$ & 2.2 & $\begin{array}{l}\text { Adjusted to include operating expenditure (Foster } \\
\text { and Briceño-Garmendia, 2010). Estimates for } \\
\text { access to water and sanitation (Hutton, 2015) } \\
\text { removed to avoid overlaps. Augmented with } \\
\text { climate adaptation costs (World Bank, 2010c). }\end{array}$ \\
\hline $\begin{array}{l}\text { Transport } \\
\text { infrastructure }\end{array}$ & $\begin{array}{l}\text { World Bank } \\
\text { (2013) }\end{array}$ & $\begin{array}{l}\text { Simple unit cost } \\
\text { estimates }\end{array}$ & 2.2 & $\begin{array}{l}\text { Adjusted to include operating expenditure (Foster } \\
\text { and Briceño-Garmendia, 2010) and mark-up on } \\
\text { capital expenditure for climate adaptation (Stern, } \\
\text { 2007). }\end{array}$ \\
\hline $\begin{array}{l}\text { Telecom } \\
\text { infrastructure }\end{array}$ & $\begin{array}{l}\text { World Bank } \\
(2013)\end{array}$ & $\begin{array}{l}\text { Simple unit cost } \\
\text { estimates }\end{array}$ & 2.2 & $\begin{array}{l}\text { Adjusted to include operating expenditure (Foster } \\
\text { and Briceño-Garmendia, 2010). }\end{array}$ \\
\hline $\begin{array}{l}\text { Ecosystems \& } \\
\text { biodiversity }\end{array}$ & $\begin{array}{l}\text { CBD } \\
(2012 a)\end{array}$ & $\begin{array}{l}\text { Intervention- } \\
\text { based needs } \\
\text { assessment }\end{array}$ & 4.4 & No major adjustments. \\
\hline Data & $\begin{array}{l}\text { Espey et al. } \\
(2015)\end{array}$ & $\begin{array}{l}\text { Intervention- } \\
\text { based needs } \\
\text { assessment }\end{array}$ & 7.5 & No major adjustments. \\
\hline
\end{tabular}


expenditure are sparse, it is not possible at this stage to determine incremental spending needs for climate change adaptation required for current expenditure. We return to this issue, which applies particularly to infrastructure and agriculture, in the discussion of results and the conclusions.

Where possible, adjustments are made to suggest how to fill gaps in available needs assessments, such as operating expenditure, missing interventions (e.g. non-communicable diseases (NCDs) in health needs assessments), or additional investment needs for climate change adaptation and mitigation, which no needs assessment integrates at present. Where two needs assessments cover the same interventions, these overlaps are removed. We find that investment needs (as opposed to outcomes) in one area tend to be only moderately affected by the level of investments made in other areas. To the extent possible, such synergies and trade-offs are quantified and considered in the aggregation of SDG investment needs.

All needs assessment results are rebased to US $\$ 2013$, harmonized to employ the same definition of incremental investments, and down-scaled to investment needs in LICs and LMICs. They are presented as non-discounted cash flows without any amortization of investments. Based on available evidence, the chapter determines the likely maximum share of private financing for each SDG investment area. With these adjustments, the harmonized investment needs can then be added across the eight SDG investment areas to yield a consistent estimate of investment needs for LICs and LMICs (Table 26). Results from needs assessments with a suitability score equal to or less than 5 are considered highly preliminary and placed in square brackets.

Since CGE models tend to be parametrized for individual economies and do not focus on the full range of SDG investments considered in this chapter, the economy-wide effects cannot be modeled dynamically at this stage. For this reason, the chapter considers the available literature to discuss how overall SDG investment needs might be affected by economy-wide effects (section 4.3.3).

\subsection{Results}

This section describes investment needs for the eight SDG investment areas, suggests how they can be aggregated across all SDGs, and considers economy-wide implications based on available evidence. It concludes by extrapolating findings to estimate incremental global SDG investment needs and outlines an SDG financing strategy.

\subsubsection{Incremental investment needs by SDG investment area}

The Annex provides a detailed summary of the principal sources for the needs assessment of each SDG investment area, classifies the main method used in each study, and describes adjustments made. Additional information, including detailed calculations and a review of all sector needs assessments that were considered for this chapter, is available in the supplementary data file.

\section{Health}

The health SDG and associated targets focus, inter alia, on ending preventable child and maternal deaths; ensuring Universal Health Coverage (UHC); and tackling major infectious and NCDs. The sector has the longest experience in developing needs assessments around global goals $(\mathrm{CMH}, 2001$; UN Millennium Project, 2005; WHO, 2010; Jamison et al., 2013; Chatham House, 2014), the latter forming the basis for this 
SDG needs assessment. WHO (2011) has prepared a separate needs assessment for NCDs, which fills a major gap in available health needs assessments. While climate change will have a significant impact on health outcomes (Watts et al., 2015; Whitmee et al., 2015), incremental investment needs are modest, because health investments are dominated by the cost of operating health systems, which are not projected to change significantly under a $2^{\circ} \mathrm{C}$ pathway (World Bank, 2010c).

Health inputs and outcomes interact strongly with those in other SDG sectors, but nonhealth interventions affect mostly investment needs for NCDs (Murray, 2015). WHO (2011) does not address such investments outside the health sector (the authors assume that disease rates remain constant in the absence of the NCD interventions), so it is likely that investment needs for individual-based interventions fall as a result of progress in non-health sectors. At this stage, we are unable to quantify this impact on NCD investment needs. However, the WHO study only considers individual-based interventions for cancer and cardio-vascular diseases, which are not strongly linked with the other SDG investments considered in this chapter (Lim et al., 2012; Roth et al., 2015). As a result, the synergies with other SDG investments are likely to be small.

Private expenditure on health currently accounts for some $20 \%$ of all health expenditure in developing countries (UNCTAD, 2014). Yet user fees for primary healthcare are incompatible with achieving UHC (Moreno-Serra and Smith, 2012; Savedoff, 2012; Jamison et al., 2013), and private expenditure for advanced medical treatment is beyond the scope of the primary healthcare focus of the SDG. For this reason, the private sector will likely not contribute significantly to filling the financing gap for the health SDG.

\section{Education}

The SDGs focus on quality education at pre-primary, primary, secondary, and postsecondary education, including adult literacy. This represents a substantial broadening of the education agenda compared with the MDGs, which focused on enrolment in primary schools. UNESCO $(2015 \mathrm{~b}, 2015 \mathrm{a})$ provides a robust needs assessment of incremental public investments for pre-primary, primary, and secondary education. Incremental investment needs for climate change adaptation in the education sector are very small (IPCC, 2014; Hughes et al., 2010). Good education outcomes depend on functioning education systems and on progress in other SDG investment areas, such as health and infrastructure (UNESCO, 2015b). However, these synergies do not have a material impact on the capital and operating costs of national education systems. These might be affected significantly, though, through the increased use of modern information and communication technologies, which hold the promise, inter alia, of making high-quality education content available at low marginal cost even for hard-toreach children (Schmidt-Traub and Sachs, 2015).

As in the health sector, household expenditure on education account for a significant share of total investments in developing countries and may be as high as $30 \%$ (UNESCO, 2015b). The UNESCO needs assessment projects only the publicly-funded share of investments in education, recognizing that a substantial share of education expenditure is made directly by households. It assumes that by 2030 out-of-pocket expenditure for education fall as a share of total education spending to reach levels observed in high-income countries today (UNESCO, 2015b, 2015a). For this reason, incremental financing for education investments reported in this chapter is public, even as the private sector continues to offer fee-based services. Such public financing is not inconsistent with private provision of education services as public funds can go towards privately-operated schools. 


\section{Social protection}

Universal access to affordable health, education, and infrastructure, as well as targeted support to smallholder farmers will address a substantial share of household expenditure by the extreme poor and reduce the likelihood of catastrophic financial shocks, e.g. owing to severe illness or injury. Moreover, SDG investments will increase economic growth and raise incomes of poor households. Nevertheless, some form of social protection system will be required for residual needs of specific groups (orphans, the disabled, victims of violence, long-term support to communities exposed to disaster, demobilization of combatants, and so forth). A number of needs assessments for social protection systems are available (ILO, 2014; Greenhill et al., 2015; Development Initiatives, 2015), but they all assume broad-based income support that does not take into account increased investments in other SDG priorities. Some (Greenhill et al., 2015; Development Initiatives, 2015) multiply the poverty gap ratio with total population, before adding mark-ups for administrative costs as well as leakage owing to poor targeting. This approach creates substantial overlap with other SDG investments. In the absence of a methodology for addressing these overlaps, available estimates of investment needs for social protection are not included in this analysis. This represents a major gap in to be closed through detailed needs assessments for social protection systems. A promising line of inquiry focuses on national assessments of targeted social protection measures that include economy-wide models to estimate the impact of SDG investments on economic growth and the incidence of extreme poverty.

\section{Food security and sustainable agriculture}

The SDGs focus on food security, improved nutrition, small-scale farmers, better research and development, and sustainable agricultural practices. As a proxy for investment needs in food security, FAO et al. (2015b) estimate income transfers needed to raise the incomes of the extreme rural poor to $\$ 20051.25$ per day. Additional investments in raising the incomes of smallholder farmers are estimated using an ICOR that is decomposed by types of agricultural investments. The former approach suffers the same shortcomings as needs assessments for social protection reviewed above and is therefore not retained for this chapter. The latter is adjusted for incremental investments needs in climate change adaptation and mitigation (UNFCCC, 2007) and overlaps with other SDG investment areas. The share of private investments in agriculture is derived from FAO et al. (2015b). The result must be considered preliminary as ICOR-based methodologies are not appropriate for robust SDG needs assessments. In particular, the methodology does not back-cast from targeted outcomes by 2030. It also does not address the broader issues of healthy nutrition and agriculture's impact on the environment. Improving needs assessments for food security, smallholder farmers, and sustainable agriculture should be a priority for future research.

\section{Infrastructure}

\section{Energy access and low-carbon energy infrastructure}

The SDGs include a goal on sustainable energy and energy access. Investment needs in energy include universal access to electricity and modern cooking fuels; and largescale power generation, transmission, and decarbonization of the energy system. These are presented separately as each category requires different financing instruments and investment strategies. The most important needs assessments for energy access were prepared by the IEA (2011), the Global Energy Assessment (GEA, 2012), and Pachauri et al. (2013). The latter is used for this assessment as it provides the most recent estimates and, in contrast to IEA (2011), includes operating expenditure for energy access, which are high owing to the need to subsidize cooking fuels for the 
poorest households. Capital investments in large-scale infrastructure are taken from World Bank (2013) and augmented by operating expenditure using Foster and Briceño-Garmendia (2010). IEA (2011) and GEA (2012) provide more sophisticated assessments, but unlike other studies considered in this chapter, they only present total rather than incremental investment needs and are difficult to disaggregate by country income group. World Bank (2013) includes investment needs for generation, transmission, and distribution of electricity, but these estimates should be treated with caution as they rely on simple, uniform cost assumptions that fail to take account of differences in energy technologies and across countries.

According to some studies (NCEC, 2014; Nelson et al., 2014) 'climate-smart' power infrastructure, combined with investments in energy efficiency (buildings, industry, transport), will not generate significant additional expenditure through to 2030 if the lower operating costs of many low-carbon technologies are fully factored in. This conclusion is broadly supported by other authors (IEA, 2012), but a number of national deep decarbonization pathways (Williams et al., 2012, 2014; SDSN and IDDRI, 2015; Bataille et al., 2016) project higher investment needs for low-carbon power infrastructure, particularly towards the end of the SDG period. In the absence of more detailed analyses, this chapter retains the NCEC (2014) analysis, which is adjusted by removing savings from lower investments in the fossil fuel supply chain, which is outside the scope of this SDG needs assessment. Similarly, savings from more compact city layout are excluded since they are subject to a high degree of uncertainty and will likely only materialize after a long time (SDSN and IDDRI, 2015). Finally, savings from lower fossil fuel consumption cannot be offset directly against higher capital expenditure needs for low-carbon power infrastructure, so this chapter does not include the savings reported in (Nelson et al., 2014). Overall, the chapter obtains investments needs for climate change mitigation of $12.6 \%$ of incremental energy investment needs. The incremental energy investment needs for climate change adaptation measures are estimated at $20 \%$ of capital investments (Stern, 2007).

UNCTAD (2014) estimates that private investment in the power sector of developing countries accounts for some $43-47 \%$ of the total. In the absence of data for operations and maintenance (O\&M), the private sector is assumed to cover $90 \%$ of O\&M costs through consumer tariffs. Private financing of improved cook stoves might be as high as $80 \%$, in line with private investments in telecommunications (UNCTAD, 2014), but it will be only $5-15 \%$ for operating expenditure (Pachauri et al., 2013). No data are available to quantify how investments in other sectors, such as agriculture and water, might affect the investment needs for meeting the energy goal. Overall, needs assessments for the energy sector cover a broad range and lack robustness in the case of power infrastructure. Such needs assessments need to be strengthened to better inform policymaking.

\section{Water and sanitation access and infrastructure}

The SDGs emphasize the importance of water and sanitation by elevating these priorities to a dedicated goal and adding water quality, water treatment, and water resources management. As with energy, investment needs for ensuring access to safe water and improved sanitation are distinguished from the broader investment needs in water management and sanitation infrastructure due to differences in technologies, delivery systems, and financing strategies. Hutton (2015) provides the most comprehensive and recent assessment of investment needs for access to water supply and sanitation. Investment needs for large scale infrastructure in this area remain to be determined, as available estimates (OECD, 2006) focus on OECD and BRICS countries only. OECD (2006) provides percentage of GDP estimates, including both capital 
and operational expenditure, for middle-income (0.54-2.6\%) and low-income (0.7$6.3 \%$ ) countries, but notes that these are based a very limited and unrepresentative subset of countries (13 MICs and 5 LICs) and therefore highly uncertain.

Incremental investment needs for climate change adaptation are drawn from UNFCCC (2007) for water supply and access to sanitation. Other authors (Ward et al., 2010; World Bank, 2010c) estimate the investment needs for flood control and the protection of water supplies. The water and sanitation sector contributes moderately to greenhouse gas emissions, but no estimates of investment needs for mitigation are available. Similarly, the impact that improved ecosystem management might have on investment needs in this sector has yet to be quantified.

Overall, needs assessment for access to water supply and sanitation are robust and demonstrate a clear production function. Yet, several gaps remain for water and sanitation-related SDG investment needs, including large-scale water and sanitation infrastructure such as full wastewater treatment and sewage networks, improved water resources management, and ending open defecation. Some of these gaps will be closed by an upcoming United Nations assessment (Hutton, personal communication). Private investment in water and sanitation is low (United Nations, 2012b), but might reach up to $20 \%$ for infrastructure (UNCTAD, 2014) and possibly $70 \%$ of O\&M.

\section{Transport infrastructure:}

Transport infrastructure, including roads, railways, and ports, represents an important investment area for achieving the SDGs. Incremental capital investment needs have been assessed (World Bank, 2013) and are augmented to include operating expenditure (Foster and Briceño-Garmendia, 2010, fig. O.3) and incremental costs for climate change adaptation (Stern, 2007). Investment needs are downscaled to LICs and LMICs on a per capita basis. The private sector is estimated to cover $32-44 \%$ of the total capital expenditure for transport infrastructure (UNCTAD, 2014). In the absence of data for developing countries a $70 \%$ private sector share for O\&M costs is assumed, as these can be recouped through gasoline taxes and in some cases toll roads. Compared with other SDG investment areas, the transport needs assessment is based on simplistic assumptions about uniform unit costs of roads, GDP growth, and the elasticity of transport investments to GDP growth. The underlying methodology does not back-cast from a desired level of per capita transport infrastructure by 2030 that might be needed to achieve the SDGs. The results must therefore be considered tentative and likely have a high margin of error.

\section{Telecommunications infrastructure}

Information and communication technologies (ICTs) can accelerate progress towards achieving the SDGs and lower associated investment needs, notably in health, education, and agriculture, but also in the energy and water sectors by helping to monitor and reduce consumption (Broadband Commission, 2014). Capital expenditure needs (World Bank, 2013), and operating expenditure are estimated separately (Foster and Briceño-Garmendia, 2010, fig. O.3). Incremental investment needs for climate change mitigation and adaptation are minimal (Fankhauser and Schmidt-Traub, 2011) (Chapter 3), so these are excluded from the analysis. Results are downscaled to LICs and LMICs on a per capita basis. The private sector is estimated to finance $41-44 \%$ of the capital expenditure for telecommunications infrastructure (UNCTAD, 2014). It is further assumed that the private sector will finance O\&M costs as they can be recovered through line rentals and user fees. The needs assessment for telecommunications in- 
frastructure is based on simple assumptions about the nature of fixed-line infrastructure needed in countries. Some of these needs have probably been replaced by mobile phone technology, while the needs assessment does not cover the investment needs for fiber optic network infrastructure. The results are therefore in need of improvement.

\section{Ecosystem services and biodiversity}

The SDGs emphasize the importance of preserving and sustainably managing marine and terrestrial ecosystems, as well as biodiversity (SDGs 14 and 15). Needs assessments for this SDG investment area are complicated by the fact that the degradation of ecosystems is often caused by a broad range of factors that cannot be addressed through narrowly defined investment programs. This in part explains the preliminary state of available needs assessments (CBD, 2012a, 2012b) that focus on the Aichi Biodiversity Targets. On balance, CBD (2012a) provides a more robust basis for an SDG needs assessment. Results are adjusted to remove overlaps with other SDG investment areas, including agriculture. Climate change is expected to have a major impact on ecosystems, but no reliable estimates are available to quantify the extent. Similarly, no adequate quantitative information could be obtained to estimate the impact that investments in other SDG investment areas might have on resource needs for ecosystems. Both represent major gaps in an SDG needs assessment. CBD (2012b, fig. 5.4) suggests that private financing may only account for some $15 \%$ of total investment needs. Available needs assessments offer scant evidence for the assumptions made and do not provide a robust production function for achieving the SDG outcomes. Their results should therefore be considered preliminary.

\section{Data for the SDGs}

Achieving the SDGs will require significant investments in data and monitoring systems. Though comparatively small in volume, these investments will be critical for success. Espey et al. (2015) provide the most comprehensive, though incomplete, estimate of investment needs in data systems. These investments will require public funding.

\section{Emergency response and humanitarian work}

The SDGs' central call to leave no one behind extends also to the victims of war, civil strife, and natural disasters who receive support in the form of humanitarian assistance. No forward-looking assessment of incremental spending needs for humanitarian work in conflict zones and in response to natural disasters is available through to 2030. Owing to the stochastic and sometimes unpredictable nature of these investment needs, the traditional methods of SDG needs assessments do not apply to this 'line item' for financing the SDGs. To get a sense of possible incremental funding needs for humanitarian assistance one can consider the ratio of unfunded needs today (OCHA, 2015) and multiply it with current spending (Global Humanitarian Assistance, 2015; OCHA, 2015). The resulting incremental funding needs for humanitarian work of \$8-23 billion do not constitute a needs assessment and should only be considered an indication of what the needs might be. This estimate does not consider spending needs for peacekeeping, which amounted to some \$9.8 billion in 2013 (Global Humanitarian Assistance, 2015). All spending on humanitarian work and emergency response is projected to be financed publicly or by private foundations. 


\subsubsection{Aggregation of results}

Table 26 consolidates incremental investment needs across the SDG investment areas in LICs and LMICs and identifies an approximate division between public and private financing sources. To assess the burden SDG investments place on national economies, they need to be expressed in purchasing power parity (\$PPP) and be divided by the country's GDP PPP to yield the share of GDP devoted to the SDGs. Available SDG needs assessments are expressed in US dollars at market exchange rates, and the detailed input analyses required to convert them into \$ PPP are unavailable. Probably the share of internationally tradable goods and services in SDG investments, e.g. many building materials (cement, steel, and bitumen), machinery, drugs and other health commodities, salaries of professionals (including engineers, doctors, and nurses), exceeds the share of tradables in most countries' GDP. Dividing SDG investment needs expressed in market prices by GDP in US dollars at market exchange rates would therefore overestimate the economic burden of achieving the SDGs in the country considered. This is because GDP (with a higher share of nontradables) will increase further when it is converted into PPP than SDG investments (with a lower share of non-tradables). For this reason, the appropriate GDP denominator for SDG investments expressed in international prices probably lies somewhere between GDP in \$ PPP and GDP in market prices. Future work should decompose SDG investments between tradables and non-tradables to compute investment needs in \$ PPP. In the meantime, this chapter reports the share of GDP that must be mobilized for incremental SDG investments as a range. The upper end of this range is obtained by dividing SDG investment needs by GDP in market prices. Dividing the needs by GDP in \$ PPP yields the lower end.

To compare projected investment needs with GDP and opportunities for domestic resource mobilization over the same period, economic growth rates must be projected through to 2030. The OECD periodically publishes long-term growth scenarios for OECD and some non-OECD countries, but these forecasts do not include LICs and LMICs. Based on Johansson et al. (2013) we project average annual real economic growth rates $2015-2030$ of $2 \%$ and $5 \%$ for high-income and upper-middle-income countries, respectively. In the absence of detailed projections, we assume convergence in per capita incomes, so LMICs are projected to grow at $7 \%$, and LICs at $8 \%$. These long-term growth rates for developing countries are high, but not unprecedented. They are plausible under an SDG pathway where substantial growth-enhancing investments are made in poverty reduction and sustainable development that will accelerate the process of convergence.

Based on these GDP growth projections LICs and LMICs will need to increase annual investments in the SDGs by some $4-11.5 \%$ of GDP $(18-45 \%$ in LICs and $3-9 \%$ in LMICs). Incremental investments needs in these countries represent $0.8-1.3 \%$ of annual world GDP over the period. Annual investment needs in the SDGs rise to 5-16\% of projected GDP in LICs and LMICs $(25-65 \%$ in LICs; $4-12 \%$ in LMICs; $1-1.5 \%$ of world GDP) if GDP growth rates are half as high as projected. If growth accelerates beyond the projected rates then the investment ratios fall correspondingly.

An extrapolation of these results to estimate global incremental investment needs requires strong assumptions (Annex 4). Incremental SDG investment needs may amount to some $\$ 2.3$ trillion per year, which corresponds to $1.5-2.5 \%$ of average world GDP over the 2015-2030 period. If GDP growth reaches only half the projected rates then the same level of SDG investments will account for $1.8-2.9 \%$ of world GDP.

Even though the analysis has several important gaps (Table 26), it is likely that the effective burden on national economies will not exceed $2.5 \%$ of GDP for two reasons: 
First, SDG investment needs comprise a high share of tradables, so the conversion towards \$ PPP will reduce the effective burden towards the lower end of the range. Second, significant efficiency gains can be expected from the simultaneous expansion of investments across such a broad range of areas and the mobilization of modern technologies for the SDGs. Wealthier countries can finance a greater share of SDG expenditure through private financing (UNCTAD, 2014) they will be able to finance at least $50 \%$ - slightly above the upper limit of private investments in LICs and LMICs of incremental global investments in the SDGs through the private sector.

As discussed in Section 4.2, it is not possible to determine by how much current public and private expenditure need to increase in response to climate change adaptation and mitigation since mark-ups can only be applied to incremental expenditure. This represents a significant gap in the analysis, but its impact on the overall results is likely modest: Incremental expenditure for climate change adaptation and mitigation of $\$_{2013} 128-133$ billion represent about one tenth of total incremental expenditure for the SDGs (Table 26). If one applies this ratio to estimated current public and private SDG expenditure of $\$ 2013509$ billion one obtains $\$ 51$ billion, which represents $3.8-4.1 \%$ of projected investment needs. 
On metrics and financing for the Sustainable Development Goals

Table 26 | Incremental annual investment needs in LICs and LMICs

Data is average annual investment needs during 2015-2030 in \$2013 billion)

\begin{tabular}{|c|c|c|c|c|c|c|c|}
\hline Investment area & $\begin{array}{l}\text { Countries } \\
\text { covered }\end{array}$ & $\begin{array}{l}\text { 'Develop- } \\
\text { ment' in- } \\
\text { vestment } \\
\text { needs }\end{array}$ & $\begin{array}{l}\text { Incremen- } \\
\text { tal climate } \\
\text { mitigation } \\
\text { and adap- } \\
\text { tation in- } \\
\text { vestment } \\
\text { needs }\end{array}$ & $\begin{array}{l}\text { Total in- } \\
\text { vestment } \\
\text { needs }\end{array}$ & $\begin{array}{c}\text { Private, } \\
\text { commercial } \\
\text { financing } \\
\text { (\% of total } \\
\text { investment } \\
\text { needs) }\end{array}$ & $\begin{array}{c}\text { Private, } \\
\text { commercial } \\
\text { financing } \\
\text { needs }\end{array}$ & $\begin{array}{l}\text { Public fi- } \\
\text { nancing } \\
\text { needs }\end{array}$ \\
\hline \multirow[t]{3}{*}{ 1. Health } & Total & $68-87$ & $1.0-1.4$ & $69-89$ & $0 \%$ & 0 & $69-89$ \\
\hline & LICs & $25-29$ & 0.3 & $25-29$ & $0 \%$ & 0 & $25-29$ \\
\hline & LMICs & $43-59$ & $0.8-1.0$ & $44-60$ & $0 \%$ & 0 & $44-60$ \\
\hline \multirow[t]{3}{*}{ 2. Education } & Total & 194 & 0 & 194 & $0 \%$ & 0 & 194 \\
\hline & LICs & 37 & 0 & 37 & $0 \%$ & 0 & 37 \\
\hline & LMICs & 157 & 0 & 157 & $0 \%$ & 0 & 157 \\
\hline $\begin{array}{l}\text { 3. Social protec- } \\
\text { tion }\end{array}$ & Total & $?$ & $?$ & $?$ & $?$ & $?$ & $?$ \\
\hline \multirow{3}{*}{$\begin{array}{l}\text { 4. Agriculture and } \\
\text { food security }\end{array}$} & Total & [125] & [22] & [148] & [51\%] & [76] & [72] \\
\hline & LICs & [61] & [6] & [67] & [51\%] & [35] & [33] \\
\hline & LMICs & [64] & {$[16]$} & [80] & [51\%] & [41] & [39] \\
\hline \multirow[t]{3}{*}{ 5.1 Energy } & Total & [265 - 289] & {$[55-57]$} & [321 - 347] & [49-50\%] & [158 - 175] & [163 - 172] \\
\hline & LICs & [73 - 82] & [14 - 15] & [88 - 97] & [47 - 48\%] & {$[41-46]$} & {$[47-51]$} \\
\hline & LMICs & [192 - 208] & {$[41-42]$} & [233 - 250] & [50 - 51\%] & [117 - 129] & [116 - 121] \\
\hline \multirow{3}{*}{$\begin{array}{l}\text { Access to elec- } \\
\text { tricity and clean } \\
\text { cooking fuels }\end{array}$} & Total & $62-83$ & $3-5$ & $66-87$ & [11 - 16\%] & {$[7-14]$} & {$[59-73]$} \\
\hline & LICs & $23-30$ & $1-2$ & $24-32$ & [13-19\%] & {$[3-6]$} & [21 - 26] \\
\hline & LMICs & $40-53$ & $2-3$ & $42-55$ & [9 - 15\%] & {$[4-8]$} & [38 - 47] \\
\hline \multirow{3}{*}{$\begin{array}{l}\text { Power infrastruc- } \\
\text { ture }\end{array}$} & Total & [203 - 207] & [52 - 53] & [255 - 259] & [59-62\%] & [151 - 161] & [99 - 104] \\
\hline & LICs & [51] & [13] & [64 - 65] & [59-62\%] & [38 - 40] & [25 - 26] \\
\hline & LMICs & [153 - 155] & [39-40] & [192 - 195] & [59 - 62\%] & [114 - 121] & [74 - 78] \\
\hline \multirow{3}{*}{$\begin{array}{l}5.2 \text { Water and } \\
\text { sanitation }\end{array}$} & Total & [28] & [14 - 17] & [42 - 45] & [0 - 20\%] & [0 - 9] & [36 - 42] \\
\hline & LICs & [7] & {$[3-4]$} & [11] & [0 - 20\%] & {$[0-2]$} & {$[9-11]$} \\
\hline & LMICs & [21] & [10 - 13] & [31 - 33] & [0 - 20\%] & {$[0-7]$} & {$[27-31]$} \\
\hline \multirow{3}{*}{$\begin{array}{l}\text { Basic water sup- } \\
\text { ply and adequate } \\
\text { sanitation }\end{array}$} & Total & 28 & $14-17$ & $42-45$ & [0 - 20\%] & {$[0-9]$} & [36 - 42] \\
\hline & LICs & 7 & $3-4$ & 11.00 & [0 - 20\%] & {$[0-2]$} & {$[9-11]$} \\
\hline & LMICs & 21 & $10-13$ & $31-33$ & [0 - 20\%] & {$[0-7]$} & [27 - 31] \\
\hline $\begin{array}{l}\text { Water and sanita- } \\
\text { tion infrastructure }\end{array}$ & Total & $?$ & $?$ & $?$ & $?$ & $?$ & $?$ \\
\hline \multirow{3}{*}{$\begin{array}{l}5.3 \text { Transport in- } \\
\text { frastructure }\end{array}$} & Total & [361] & [35] & [396] & [52 - 57\%] & [205 - 228] & [169 - 192] \\
\hline & LICs & [90] & [9] & [99] & [52 - 57\%] & [51 - 57] & [42 - 48] \\
\hline & LMICs & [271] & [26] & [298] & [52 - 57\%] & [154 - 171] & [127 - 144] \\
\hline \multirow{3}{*}{$\begin{array}{l}5.4 \text { Telecommuni- } \\
\text { cations infrastruc- } \\
\text { ture }\end{array}$} & Total & [189] & {$[0]$} & [189] & [54 - 86\%] & [102 - 163] & [26 - 87] \\
\hline & LICs & [47] & [0] & [47] & [54 - 86\%] & [25 - 40] & [7 - 22] \\
\hline & LMICs & [142] & [0] & [142] & [54 - 86\%] & [77 - 122] & [20 - 65] \\
\hline
\end{tabular}




\begin{tabular}{|c|c|c|c|c|c|c|c|}
\hline Investment area & $\begin{array}{l}\text { Countries } \\
\text { covered }\end{array}$ & $\begin{array}{l}\text { 'Develop- } \\
\text { ment' in- } \\
\text { vestment } \\
\text { needs }\end{array}$ & $\begin{array}{l}\text { Incremen- } \\
\text { tal climate } \\
\text { mitigation } \\
\text { and adap- } \\
\text { tation in- } \\
\text { vestment } \\
\text { needs }\end{array}$ & $\begin{array}{l}\text { Total in- } \\
\text { vestment } \\
\text { needs }\end{array}$ & $\begin{array}{c}\text { Private, } \\
\text { commercial } \\
\text { financing } \\
\text { (\% of total } \\
\text { investment } \\
\text { needs) }\end{array}$ & $\begin{array}{c}\text { Private, } \\
\text { commercial } \\
\text { financing } \\
\text { needs }\end{array}$ & $\begin{array}{l}\text { Public fi- } \\
\text { nancing } \\
\text { needs }\end{array}$ \\
\hline \multirow{3}{*}{$\begin{array}{l}\text { 6. Ecosystems, in- } \\
\text { cluding biodiver- } \\
\text { sity }\end{array}$} & Total & [11 - 28] & $?$ & [11 - 28] & [15\%] & [2 - 4] & [9 - 24] \\
\hline & LICs & {$[3-7]$} & $?$ & {$[3-7]$} & [15\%] & {$[0-1]$} & {$[2-6]$} \\
\hline & LMICs & {$[8-21]$} & $?$ & {$[8-21]$} & [15\%] & {$[1-3]$} & [7 - 18] \\
\hline \multirow{3}{*}{$\begin{array}{l}\text { 7. Data for the } \\
\text { SDGs }\end{array}$} & Total & 0.5 & 0 & 0.5 & $0 \%$ & 0 & 0.5 \\
\hline & LICs & 0.3 & 0 & 0.3 & $0 \%$ & 0 & 0.3 \\
\hline & LMICs & 0.2 & 0 & 0.2 & $0 \%$ & 0 & 0.2 \\
\hline $\begin{array}{l}\text { 8. Emergency re- } \\
\text { sponse and hu- } \\
\text { manitarian work }\end{array}$ & Total & {$[8-23]$} & $?$ & {$[8-23]$} & [0\%] & [0] & [8 - 23] \\
\hline \multirow{3}{*}{$\begin{array}{l}\text { All SDG invest- } \\
\text { ment areas*** }\end{array}$} & Total & [1251 - 1327] & [128 - 133] & [1378 - 1459] & [39 - 45\%] & [543 - 654] & [805 - 836] \\
\hline & LICs & {$[343-360]$} & [33 - 35] & [376 - 394] & [40 - 46\%] & [152 - 181] & [213 - 224] \\
\hline & LMICs & [900 - 944] & [95 - 98] & [995 - 1042] & [39- 45\%] & [390 - 473] & [569 - 604] \\
\hline
\end{tabular}

Principal sources: health (Jamison et al., 2013; WHO, 2011); education (UNESCO, 2015b, 2015a); agriculture and food security (FAO et al., 2015b); energy access and infrastructure (Pachauri et al., 2013; World Bank, 2013); water and sanitation access (Hutton, 2015); transport and telecommunications infrastructure (World Bank, 2013); ecosystems (CBD, 2012a); data (Espey et al., 2015).

Notes: Estimates reported in sources have been adjusted for this table (see Annex and supplementary online data file). They have also been rounded and may not add up exactly. Numbers in square brackets derive from studies with a suitability score of 5 or less. They are particularly uncertain or incomplete and subject to refinement.

* Emergency response and humanitarian work will be entirely funded by concessional public international financing and cannot be disaggregated by income group.

** This excludes several SDG investment needs identified in the chapter, including social protection, largescale water supply and sanitation infrastructure, incremental investment needs for climate change mitigation and adaptation for ecosystems, and changes to the composition and scale of current infrastructure investments. The total does not equal sum of LICs and LMICs since investment needs for emergency response and humanitarian work are allocated to total only. 


\subsubsection{Synergies and economy-wide effects}

Investment needs for education and health systems (with the notable exception of NCDs) are relatively invariant to investments in other areas. Meanwhile, investments in infrastructure, agriculture, climate change mitigation and adaptation, and other areas exhibit substantial synergies (McCollum et al., 2013). Available studies and data are insufficient to quantify such relationships in global sector needs assessments. Future work should focus on integrated country-level modeling to quantify positive and negative synergies across SDG investments (e.g. by expanding the MAMS tool) as well as global and regional modeling of integrated long-term pathways for achieving the SDGs.

In addition, at least three economy-wide effects of SDG investments must be considered. First, Bourguignon, Diaz-Bonilla, and Lofgren (2008) show for Ethiopia that a 10year MDG investment program will increase the compound annual growth in nominal wages by up to $1-5 \%$ per year, depending on the level of education. The authors underscore the complexity of projecting mid-term wage developments since they depend on the demand side (government programs and evolution of the private sector) and the supply side (the education system). Recent applications of MAMS (Gable et al., 2015; Levin, 2015a, 2015b) provide no further guidance on the quantitative impact of changes in real wages. Some SDG needs assessments suggest that real wages will increase (Jamison et al., 2013; UNESCO, 2015a), but this question needs to be considered more systematically.

Second, economy-wide models, such as MAMS identify trade-offs between tax-financed investments, the intertemporal effects of debt financing, and economic growth. However, the quantitative effects depend on the specifications of the models (notably the extent to which supply-side effects through better health, education, and infrastructure are included) and the structure of the economy. No economy-wide tools exist to project the impacts of the full spectrum of SDG investments over a 15-year period. As a result, it is impossible to draw general conclusions from the impact of increased SDG investments on economic growth.

Third, inflows of foreign currency (e.g. in the form of aid) as well as the composition of domestic investments may lead to a real exchange rate appreciation and thereby shift domestic investments away from high-growth export sectors towards lower-growth domestic consumption. MAMS applications (Bourguignon et al., 2008; Gable et al., 2015; Levin, 2015a, 2015b) suggest that increases in foreign grants will lead to a significant appreciation of the real exchange rate and a substantial weakening of countries' export sectors. Yet, IMF analyses conclude that under prudential macroeconomic management, the positive effects of substantial increases in ODA flows will outweigh adverse consequences (IMF, 2008; Mongardini and Samake, 2009; Prati et al., 2003). These differences in conclusions stem largely from differences in treating the supply-side effects of increased foreign currency inflows and in the granularity with which macroeconomic policies are modeled. On balance, there is no clear-cut answer to how foreign currency inflows will affect economic growth and macroeconomic stability. Better answers are needed drawing on broader and more granular economy-wide models.

Other important issues concern the economy-wide effects of greater efficiency in government expenditure and technological change. All in all, economy-wide effects of SDG investments are likely to be substantial and highly country specific, but they remain relatively poorly understood. This points to three important lines of future inquiry: (1) Broaden country-level macroeconomic modeling tools, such as MAMS, to include a fuller set of SDG investments as has recently been initiated by the World Bank (Gable et al., 2015); (2) Strengthen the integrated modeling of sector interactions and economy-wide effects at the global and regional level; and (3) Improve our understanding 
4 - Investment needs to achieve SDGs in low- and lower-middle-income countries

of how the transformational role of modern technologies can be harnessed to accelerate progress towards the SDGs, reduce associated investment needs, and improve the integration of available strategies.

\subsubsection{Stylized financing analysis}

A detailed financing analysis for incremental SDG investment needs is beyond the scope of this chapter. Below we outline a tentative and indicative first cut at what such an analysis might look like for LICs and LMICs. Detailed assumptions, sources, and calculations are provided in the supplementary data file.

SDSN (2015b) proposes that countries reach the following benchmarks for government revenues as a share of GDP by 2020: Least-developed Countries (LDCs): 17\%; other LICs: $20 \%$; LMICs: $22 \%$; UMICs and HICs: at least $24 \%$. The share of central government expenditure devoted to the SDGs is further assumed to rise from some $40 \%$ today to $60 \%$ in LICs and $66 \%$ in LMICs. Using the assumptions on economic growth rates through to 2030 described under Section 4.3.2, total central government revenues dedicated to the SDGs each year are estimated to reach $\$ 2013153$ billion in LICs and $\$ 20132,442$ billion in LMICs by 2030, equal to an incremental effort over 2014 central government SDG expenditure of $\$ 127$ billion in LICs and $\$ 1,959$ billion in LMICs. This corresponds to average incremental central government revenues dedicated to the SDGs for each year from 2015 to 2030 of $\$ 61$ billion in LICs and \$984 billion in LMICs. Subtracting the annual public financing needs identified in Table 26 yields a financing gap of \$152-163 billion per year on average from 2015 to 2030 in LICs, while LMICs are predicted to cover investment on average over the period. However, these countries may require international co-financing at the beginning of the SDG period, when investments needs are likely to rise faster than countries' ability to mobilize private and domestic public resources.

To assess whether the international financing gap can reasonably be closed, one can project volumes of concessional international public finance, including ODA, through to 2030. Three sources of international public financing can be considered (SchmidtTraub and Sachs, 2015): (1) Member countries of the OECD Development Assistance Committee (DAC) may increase financing to $0.7 \%$ of their GNI by 2030 , as called for in the Addis Ababa Action Agenda (United Nations, 2015b); (2) non-OECD DAC HICs may match the commitments by DAC member countries; and (3) UMICs might increase their financing from currently $0.1 \%$ of their GNI (OECD, 2014) to $0.3 \%$ of GNI by 2030 . Combining these three sources yields an incremental average $\$_{2013} 240$ billion per year through to 2030, which far exceeds the net financing gap for the SDGs. We note that the assumptions on mobilizing international public financing are conservative since they exclude the role of private philanthropy as well as the potential of non-concessional international public finance (such as Other Official Flows, OOF) to fill parts of the financing gap.

Overall, this simple and tentative financing analysis suggests that the incremental SDG financing needs require significant but manageable increases in public expenditure and can be met within existing political commitments. Yet, the results are highly sensitive to projections of GDP. Lower GDP growth rates would reduce domestic resource mobilization and thereby increase the external financing gap. A more sophisticated financing analysis is needed to, inter alia, consider domestic resource mobilization in greater detail; include debt financing; distinguish between concessional and non-concessional international public finance; endogenize economic growth assumptions; and consider the microeconomic implications of increased domestic resource mobilization on the achievement of the SDGs. 


\subsection{Conclusions and discussion}

Drawing on the experience of MDG sector needs assessments and their effectiveness, this chapter translates the 17 SDGs into eight SDG investment areas, proposing an analytical framework and a preliminary suitability score for assessing the quality and robustness of each needs assessment. The results show that needs assessments in the social sectors (health and education), access to water and sanitation, and access to energy services tend to be strongest, while needs assessments for the environment, large-scale infrastructure, agriculture, and food security are weakest. Investment needs for social protection remain to be estimated, as do the estimates for private business investment needed to achieve economic growth targets. The framework also underscores that many SDG needs assessments do not integrate climate change adaptation and mitigation. Where possible, the chapter adjusts needs assessment results to make them comparable, fill gaps, remove overlaps, and consider the effect of synergies across investment areas.

In summary, incremental SDG financing needs appear manageable at $\$ 1.4$ trillion in LICs and LMICs. Global investment needs might be $1.5-2.5 \%$ of world GDP. These headline figures hide different types of financing needs. Infrastructure will require trillions of incremental US dollars per year, a large share of this should be financed through private and non-concessional public financing. Incremental investment needs for health, education, food security, and other areas are in the order of tens of billions, but they overwhelmingly require concessional public financing.

At least three priorities for future research emerge from this first comprehensive but preliminary SDG needs assessment:

1. Strengthen and update sector needs assessments particularly for agriculture and food security, infrastructure, ecosystem services, and social protection. Methodologies should be reviewed to ensure that the results can guide national and global discussions on how the SDGs can be achieved and financed. Using the analytical framework developed in this chapter, sectoral assessments can analyze changes in the composition and volume of total SDG investments (as opposed to only incremental investments), integrate investment needs related to climate change, remove overlaps, fill gaps, and consider how synergies and trade-offs may be addressed. Needs assessments should also disaggregate between investments in tradables and non-tradables so that their results can be expressed in \$ PPP. They must also pay greater attention to understanding how quickly private and public investments can be scaled up to achieve the SDGs by 2030 . Periodically updated sector needs assessments should be consolidated to guide global policy discussions on financing the SDGs.

2. Develop country needs assessments and integrate economic growth modelling. The sector needs assessments can inform more detailed needs assessments at the country level that should take into account synergies across SDG investment areas, investments in business capital, and economywide effects. Integrated assessment tools and expanded computable-general equilibrium models (such as the World Bank's MAMS) that span the full range of SDG investments are needed to support country-level assessments of investment needs. They should also integrate economic growth modeling and sector modeling to understand how countries can meet not only the sector targets but also aggregate improvements in income and employment consistent with the SDGs. Such integrated analyses will also help compare results of SDG 
4 - Investment needs to achieve SDGs in low- and lower-middle-income countries

needs assessments with estimates of inclusive wealth or genuine savings required to achieve the SDGs.

3. Develop a robust financing strategy. The indicative SDG financing framework identified in this chapter needs to be improved by assessing total (as opposed to incremental) investment needs for the SDGs; developing a framework for domestic resource mobilization, including through government bonds, and an associated debt sustainability analysis; and determining the potential for non-concessional international public finance and the role of the multi-lateral development banks. It must be integrated into country-level growth models to obtain more robust estimates of the potential for long-term domestic resource mobilization and to support the design of supportive macroeconomic policies.

\section{Acknowledgements}

The author thanks the following people for advice and support: Manos Antoninis, Chandrika Bahadur, Amar Bhattacharya, Jessica Espey, Vivien Foster, Arif Husain, Guy Hutton, Ronda Jordan, Pavel Kabat, Homi Kharas, Marcus Manuel, John McArthur, Hafiz Mirza, Shonali Pachauri, Nora Selmet, Ekko van lerland, Paul Watkiss, and James Zhan. David Durand-Delacre has provided outstanding research assistance. This work is part of an extended project with Jeffrey Sachs and other SDSN colleagues to assess the quantitative trajectories of investments nationally and globally to achieve sustainable development. 


\section{Annex 4. Supplementary material}

Table 27 | Principal sources for needs assessments and adjustments made

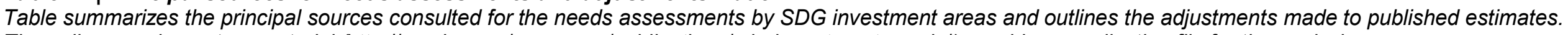

The online supplementary material (http://unsdsn.org/resources/publications/sdg-investment-needs/) provides a replication file for the analysis.

\begin{tabular}{|c|c|c|c|c|}
\hline $\begin{array}{l}\text { SDG Invest- } \\
\text { ment Area } \\
\text { (source) }\end{array}$ & Coverage and key gaps & Quality assessment & $\begin{array}{l}\text { Results reported } \\
\text { in study (Period } \\
\text { average in } \$ \text { bil- } \\
\text { lions p.a.) }\end{array}$ & Adjustments made in this chapter \\
\hline $\begin{array}{l}\text { Health (Jamison } \\
\text { et al., 2013) }\end{array}$ & $\begin{array}{l}\text { Coverage: Universal Health Cov- } \\
\text { erage (UHC) and a 'Grand Con- } \\
\text { vergence' in health (Reduction in } \\
\text { the burden of infections and } \\
\text { RMNCH disorders in most high- } \\
\text { mortality LICs and LMICs down to } \\
\text { the rates presently seen in the } \\
\text { best performing MICs). Gaps: } \\
\text { Non-communicable diseases; epi- } \\
\text { demic preparedness and response } \\
\text { plans. }\end{array}$ & $\begin{array}{l}\text { Intervention-based needs assessment: } 1 \\
\text { Goal-based analysis: } 1 \\
\text { Comprehensive coverage of interventions: } 1 \\
\text { Peer-reviewed assessment: } 1 \\
\text { Clear identification of inputs to address overlaps/gaps with other sectors: } 1 \\
\text { Inclusion of operating and capital expenditure: } 1 \\
\text { Disaggregation of results by low- and lower-middle-income countries: } 1 \\
\text { Inclusion of results from dynamic economy-wide models: } 0 \\
\text { Consideration of investment needs for climate change adaptation and mitiga- } \\
\text { tion: } n \text { /a } \\
\text { Suitability score (out of } \mathbf{1 0 )}: \mathbf{8 . 8}\end{array}$ & $\begin{array}{l}\text { \$61 (2016-2025)- } \\
\text { \$80 (2026-2035): } \\
\text { LICs: } \\
\text { \$23 (2016-2025) - } \\
\text { \$27 (2026-35); } \\
\text { LMICs: } \\
\text { \$38 (2016-2025) - } \\
\text { \$53 (2026-2035) }\end{array}$ & $\begin{array}{l}\text { Rebased to } \$ 2013 \text { yielding development investment } \\
\text { needs of } \$ 63-83 \text { billion (LICs: } \$ 24-28 \text { billion, } \\
\text { LMICs: } \$ 39-55 \text { billion). Adaptation investment } \\
\text { needs of } \$ 20052 \text { billion per year (rebased to } \$ 2013) \\
\text { from Pandey ( } 2010 \text { ) are added. Combined with } \\
\text { WHO (2011) for NCDs, this yields a total of } \$ 69-89 \\
\text { billion (LICs: } \$ 25-29 \text { billion, LMICs: } \$ 44-60 \text { bil- } \\
\text { lion). }\end{array}$ \\
\hline $\begin{array}{l}\text { Education } \\
\text { (UNESCO, } \\
2015 b, 2015 a)\end{array}$ & $\begin{array}{l}\text { Coverage: Achieving universal } \\
\text { pre-primary, primary, lower and } \\
\text { upper secondary education of } \\
\text { good quality. Gaps: Tertiary edu- } \\
\text { cation; skills for work; adult liter- } \\
\text { acy; scholarships; teacher training. }\end{array}$ & $\begin{array}{l}\text { Intervention-based needs assessment: } 1 \\
\text { Goal-based analysis: } 1 \\
\text { Comprehensive coverage of interventions: } 1 \\
\text { Peer-reviewed assessment: } 0 \\
\text { Clear identification of inputs to address overlaps/gaps with other sectors: } 1 \\
\text { Inclusion of operating and capital expenditure: } 1 \\
\text { Disaggregation of results by low- and lower-middle-income countries: } 1 \\
\text { Inclusion of results from dynamic economy-wide models: } 0 \\
\text { Consideration of investment needs for climate change adaptation and mitiga- } \\
\text { tion: } \mathrm{n} / \mathrm{a} \\
\text { Suitability score (out of } \mathbf{1 0}): \mathbf{7 . 5}\end{array}$ & $\begin{array}{l}\text { Increase in total } \\
\text { costs: } \\
\text { \$191 }(2015-2030) \\
\text { LICs: } \$ 36 \\
\text { LMICs: } \$ 155 \\
\text { Financing gap: } \\
\text { \$39 }(2015-2030) \\
\text { LICs: } \$ 21 \\
\text { LMICs: } \$ 18 .\end{array}$ & $\begin{array}{l}\text { Rebased to } \$ 2013 \text {, yielding } \$ 194 \text { billion (LICs: } \$ 37 \\
\text { billion; LMICs: } \$ 157 \text { billion) in investment needs } \\
\text { incremental to total current spending. Climate } \\
\text { change-related investments are assumed to be } \\
\text { zero. }\end{array}$ \\
\hline $\begin{array}{l}\text { Social Protec- } \\
\text { tion (Greenhill et } \\
\text { al., 2015) } \\
\text { Note: Develop- } \\
\text { ment Initiatives } \\
\text { (2015) uses a } \\
\text { similar method- } \\
\text { ology and ob- } \\
\text { tains similar re- } \\
\text { sults. }\end{array}$ & $\begin{array}{l}\text { Coverage: A 'Basic Social Com- } \\
\text { pact' comprising basic social pro- } \\
\text { tection, universal health care cov- } \\
\text { erage and universal primary and } \\
\text { secondary education. Gaps: The } \\
\text { study does not consider social pro- } \\
\text { tection needs of groups other than } \\
\text { the extreme poor, except for uni- } \\
\text { versal free access to health and } \\
\text { education. See below and text for } \\
\text { comments on methodology. }\end{array}$ & $\begin{array}{l}\text { Comprehensive coverage of interventions: } 0 \\
\text { Clear identification of inputs to address overlaps/gaps with other sectors: } 0 \\
\text { Intervention-based needs assessment: } 0 \\
\text { Goal-based analysis: } 1 \\
\text { Peer-reviewed assessment: } 0 \\
\text { Inclusion of operating and capital expenditure: } 1 \\
\text { Disaggregation of results by low- and lower-middle-income countries: } 1 \\
\text { Consideration of investment needs for climate change adaptation and mitiga- } \\
\text { tion: } 0 \\
\text { Inclusion of results from dynamic economy-wide models: } 0 \\
\text { Suitability score (out of } 10): 3.3\end{array}$ & $\begin{array}{l}\text { \$626 (2011-2030) } \\
\text { LICs: \$148 } \\
\text { LMICs: \$478. } \\
\text { Of which, basic } \\
\text { social protection } \\
\text { (LICs: } \$ 42 \text { LMICs: } \\
\text { \$40); education } \\
\text { (LICs: \$32 } \\
\text { LMICs: \$168); } \\
\text { and health } \\
\text { (LICs: } \$ 74 \\
\text { LMICs: } \$ 269)\end{array}$ & $\begin{array}{l}\text { Investments needs for health and education are al- } \\
\text { ready covered in the corresponding SDG invest- } \\
\text { ment areas. Remaining investments in social pro- } \\
\text { tection overlap significantly with other SDG invest- } \\
\text { ments (see text) and are therefore not included in } \\
\text { the summary table. A more targeted methodology I } \\
\text { needed for this needs assessment. }\end{array}$ \\
\hline
\end{tabular}




\begin{tabular}{|c|c|c|c|c|}
\hline $\begin{array}{l}\text { SDG Invest- } \\
\text { ment Area } \\
\text { (source) }\end{array}$ & Coverage and key gaps & Quality assessment & $\begin{array}{l}\text { Results reported } \\
\text { in study (Period } \\
\text { average in } \$ \text { bil- } \\
\text { lions p.a.) }\end{array}$ & Adjustments made in this chapter \\
\hline $\begin{array}{l}\text { Food Security } \\
\text { and Agriculture } \\
\text { (FAO et al., } \\
2015 \mathrm{~b} \text { ) }\end{array}$ & $\begin{array}{l}\text { Coverage: Reaching 'zero hunger,' } \\
\text { defined as less than } 5 \% \text { of the } \\
\text { population as undernourished and } \\
\text { ending extreme poverty. Gaps: } \\
\text { Addressing micro-nutritional } \\
\text { needs; climate-resilient agriculture; } \\
\text { elimination of agricultural export } \\
\text { subsidies; investment needs for } \\
\text { commercial agriculture. }\end{array}$ & $\begin{array}{l}\text { Comprehensive coverage of interventions: } 1 \\
\text { Clear identification of inputs to address overlaps/gaps with other sectors: } 0 \\
\text { Intervention-based needs assessment: } 0 \\
\text { Goal-based analysis: } 0 \\
\text { Peer-reviewed assessment: } 0 \\
\text { Inclusion of operating and capital expenditure: } 1 \\
\text { Disaggregation of results by low- and lower-middle-income countries: } 1 \\
\text { Consideration of investment needs for climate change adaptation and mitiga- } \\
\text { tion: } 0 \\
\text { Inclusion of results from dynamic economy-wide models: } 1 \\
\text { Suitability score (out of } 10 \text { ): } 4.4\end{array}$ & $\begin{array}{l}\text { \$245 (2016-2030) } \\
\text { Breakdown: } \$ 100 \\
\text { for PGT and } \$ 145 \\
\text { for pro-poor in- } \\
\text { vestments in agri- } \\
\text { culture }\end{array}$ & $\begin{array}{l}\text { Investments in the PGT ( } \$ 100 \text { billion) and for rural } \\
\text { electrification and roads ( } \$ 20 \text { billion) are removed } \\
\text { to avoid overlaps. The total is disaggregated by in- } \\
\text { come group based on country-level data provided } \\
\text { in the study, for total 'development' needs of } \$ 125 \\
\text { billion per year (LICs: } \$ 61, \text { LMICS: } \$ 64 \text { ). A } 2 \% \\
\text { mark-up is applied for incremental climate change } \\
\text { adaptation needs (UNFCCC, } 2007 \text { ), and } \$ 35 \text { bil- } \\
\text { lion for mitigation ( } \$ 15 \text { for CO } \text { CO }_{2} \text { removal by sinks + } \\
\$ 20 \text { for reducing non-CO2 emissions from agricul- } \\
\text { ture by } 10 \% \text { ) added, yielding a total of } \$ 148 \text { billion } \\
\text { per year (LICs: } \$ 67, \text { LMICs: } \$ 80 \text { ) }\end{array}$ \\
\hline $\begin{array}{l}\text { Energy Access } \\
\text { (Pachauri et al., } \\
\text { 2013) }\end{array}$ & $\begin{array}{l}\text { Coverage: Universal access to } \\
\text { modern energy in rural areas; uni- } \\
\text { versal access to clean cooking. } \\
\text { Gaps: Increase in renewable en- } \\
\text { ergy; energy efficiency measures; } \\
\text { energy R\&D; urban electrification. }\end{array}$ & $\begin{array}{l}\text { Comprehensive coverage of interventions: } 1 \\
\text { Clear identification of inputs to address overlaps/gaps with other sectors: } 1 \\
\text { Intervention-based needs assessment: } 1 \\
\text { Goal-based analysis: } 1 \\
\text { Peer-reviewed assessment: } 1 \\
\text { Inclusion of operating and capital expenditure: } 1 \\
\text { Disaggregation of results by low- and lower-middle-income countries: } 0 \\
\text { Consideration of investment needs for climate change adaptation and mitiga- } \\
\text { tion: } 0 \\
\text { Inclusion of results from dynamic economy-wide models: } 1 \\
\text { Suitability score (out of } 10 \text { ): } 7.8\end{array}$ & $\begin{array}{l}\text { \$65-86 (2010- } \\
\text { 2030) Breakdown: } \\
\$ 12.7-18.2 \text { for uni- } \\
\text { versal access to } \\
\text { electricity }+\$ 52.3- \\
67.8 \text { for universal } \\
\text { access to clean } \\
\text { cooking. }\end{array}$ & $\begin{array}{l}\text { Rebased to } \$ 2013 \text { and scaled down to LICs and } \\
\text { LMICs on a per capita basis, yielding } \$ 54-71 \text { billion } \\
\text { per year (LICs: } \$ 19-26 \text {, LMICS: } \$ 34-45) \text {. A } 20 \% \\
\text { mark-up on capital expenditure is included for cli- } \\
\text { mate change adaptation (Stern, 2007). For mitiga- } \\
\text { tion, Pachauri et al. (2013) project that meeting the } \\
\text { goals outlined in the study will not have significant } \\
\text { impact on greenhouse gas emissions. This results } \\
\text { in investment needs of } \$ 64-85 \text { billion per year } \\
\text { (LICs: } \$ 19-26 \text {, LMICs: } \$ 34-45 \text { ). This is combined } \\
\text { with World Bank (2013) for a final estimate for en- } \\
\text { ergy of } \$ \mathbf{3 0 8 - 3 3 3} \text { billion per year (LICs: } \$ \mathbf{8 4 - 9 3} \text {, } \\
\text { LMICs: } \$ \mathbf{2 2 4 - 2 4 0 )}\end{array}$ \\
\hline $\begin{array}{l}\text { Power Infra- } \\
\text { structure (World } \\
\text { Bank, 2013) }\end{array}$ & $\begin{array}{l}\text { Coverage: Infrastructure needs for } \\
\text { power generation, transmission } \\
\text { and distribution. Gaps: Operation } \\
\text { and maintenance expenditure for } \\
\text { infrastructure. }\end{array}$ & $\begin{array}{l}\text { Comprehensive coverage of interventions: } 0 \\
\text { Clear identification of inputs to address overlaps/gaps with other sectors: } 1 \\
\text { Intervention-based needs assessment: } 1 \\
\text { Goal-based analysis: } 0 \\
\text { Peer-reviewed assessment: } 0 \\
\text { Inclusion of operating and capital expenditure: } 0 \\
\text { Disaggregation of results by low- and lower-middle-income countries: } 0 \\
\text { Consideration of investment needs for climate change adaptation and mitiga- } \\
\text { tion: } 0 \\
\text { Inclusion of results from dynamic economy-wide models: } 0 \\
\text { Suitability score (out of } \mathbf{1 0 ) :} \mathbf{2 . 2}\end{array}$ & $\$ 228(2012-2030)$ & $\begin{array}{l}\text { Operating expenditure added assuming that O\&M } \\
\text { represent } 34.6 \% \text { of total power infrastructure costs } \\
\text { (Foster and Briceño-Garmendia, } 2010 \text {, fig. O.3). } \\
\text { Investment needs are then rebased to } \$ 2013 \text {. To } \\
\text { avoid overlaps, the estimated } \$ 15-21 \text { billion } \\
\text { needed for access to rural electrification estimated } \\
\text { by Pachauri et al. (2013) are removed. Estimates } \\
\text { are then scaled down to LICs and LMICs on a per } \\
\text { capita basis, for a total of } \$ 189 \text { billion per year } \\
\text { (LICs: } \$ 47 \text { billion, LMICs: } \$ 142 \text { billion). A } 20 \% \\
\text { mark-up on capex is included for climate change } \\
\text { adaptation (Stern, } 2007) \text {, yielding a total of } \$ 244- \\
248 \text { billion per year (LICs: } \$ 61-62 \text {, LMICs: } \$ 183- \\
186 \text { ). }\end{array}$ \\
\hline
\end{tabular}




\begin{tabular}{|c|c|c|c|c|}
\hline $\begin{array}{l}\text { SDG Invest- } \\
\text { ment Area } \\
\text { (source) }\end{array}$ & Coverage and key gaps & Quality assessment & $\begin{array}{l}\text { Results reported } \\
\text { in study (Period } \\
\text { average in } \$ \text { bil- } \\
\text { lions p.a.) }\end{array}$ & Adjustments made in this chapter \\
\hline $\begin{array}{l}\text { Water and Sani- } \\
\text { tation (Hutton, } \\
\text { 2015) }\end{array}$ & $\begin{array}{l}\text { Coverage: Achieving universal ac- } \\
\text { cess to 'basic' water supply and to } \\
\text { 'adequate sanitation,' as well as } \\
\text { ending open defecation. Gaps: Ac- } \\
\text { cess to 'safe' water supply, } \\
\text { wastewater treatment; integrated } \\
\text { water management; community } \\
\text { participation in water manage- } \\
\text { ment; water-use efficiency } \\
\text { measures; capacity-building. }\end{array}$ & $\begin{array}{l}\text { Comprehensive coverage of interventions: } 1 \\
\text { Clear identification of inputs to address overlaps/gaps with other sectors: } 1 \\
\text { Intervention-based needs assessment: } 1 \\
\text { Goal-based analysis: } 1 \\
\text { Peer-reviewed assessment: } 0 \\
\text { Inclusion of operating and capital expenditure: } 1 \\
\text { Disaggregation of results by low- and lower-middle-income countries: } 1 \\
\text { Consideration of investment needs for climate change adaptation and mitiga- } \\
\text { tion: } 0 \\
\text { Inclusion of results from dynamic economy-wide models: } 0 \\
\text { Suitability score (out of } 10): 6.7\end{array}$ & $\begin{array}{l}\$ 49(2015-2030) \\
\text { Breakdown: } \$ 17.5 \\
\text { for universal ac- } \\
\text { cess to basic wa- } \\
\text { ter; } \$ 31.5 \text { for uni- } \\
\text { versal access to } \\
\text { adequate sanita- } \\
\text { tion. }\end{array}$ & $\begin{array}{l}\text { Rebased to } \$ 2013 \text { and scaled down to LICs and } \\
\text { LMICs using data provided by the author (Hutton, } \\
\text { personal communication), yielding } \$ 28 \text { billion per } \\
\text { year (LICs: } \$ 7 \text {, LMICs: } \$ 21 \text { ). For adaptation, a } \\
25 \% \text { mark-up is applied to capital expenditure } \\
\text { (UNFCCC, 2007). Incremental costs for mitigation } \\
\text { are assumed to be minimal and are not taken into } \\
\text { account. This yields a total of } \$ 34 \text { billion per year } \\
\text { (LICs: } \$ 8 \text {, LMICs: } \$ 25 \text { ). Combined with residual in- } \\
\text { vestment needs from World Bank (2013), this } \\
\text { leaves a total of \$43-46 billion per year (LICs: } \\
\text { \$11, LMICs: } 32-34) \text {. }\end{array}$ \\
\hline $\begin{array}{l}\text { Water and Sani- } \\
\text { tation Infrastruc- } \\
\text { ture (World } \\
\text { Bank, 2013) }\end{array}$ & $\begin{array}{l}\text { Coverage: Infrastructure invest- } \\
\text { ments for potable water supply } \\
\text { and wastewater treatment. Gaps: } \\
\text { Wider water and sanitation infra- } \\
\text { structure, including operation and } \\
\text { maintenance expenditure for infra- } \\
\text { structure. }\end{array}$ & $\begin{array}{l}\text { Comprehensive coverage of interventions: } 0 \\
\text { Clear identification of inputs to address overlaps/gaps with other sectors: } 1 \\
\text { Intervention-based needs assessment: } 1 \\
\text { Goal-based analysis: } 0 \\
\text { Peer-reviewed assessment: } 0 \\
\text { Inclusion of operating and capital expenditure: } 0 \\
\text { Disaggregation of results by low- and lower-middle-income countries: } 0 \\
\text { Consideration of investment needs for climate change adaptation and mitiga- } \\
\text { tion: } 0 \\
\text { Inclusion of results from dynamic economy-wide models: } 0 \\
\text { Suitability score (out of } \mathbf{1 0}): \mathbf{2 . 2}\end{array}$ & $\$ 61(2012-2030)$ & $\begin{array}{l}\text { Operating expenditure is estimated based on as- } \\
\text { sumption that O\&M represent } 32 \% \text { of total infra- } \\
\text { structure costs (Foster and Briceño-Garmendia, } \\
2010 \text {, fig. O.3). For all countries covered in the } \\
\text { study, this yields a total of } \$ 50 \text { billion. Once scaled } \\
\text { down to LICs and LMICs on a per capita basis to } \\
\text { yield } \$ 29 \text { billion per year (LICs: } \$ 7 \text { billion, LMICs: } \\
\$ 22 \text { billion). To avoid overlaps, estimates from Hut- } \\
\text { ton (2015) are subtracted, leaving an adjusted es- } \\
\text { timate of } \$ 1 \text { billion per year (LICs: } \$ 0 \text {, LMICs: } \$ 1 \text { ). } \\
\text { Adaptation costs are based on World Bank } \\
\text { (2010c), and scaled down on a per capita basis, } \\
\text { yielding incremental investment needs for adapta- } \\
\text { tion of } \$ 8-11 \text { billion per year (LICs: } \$ 2-3 \text {, LMICs: } \\
\$ 6-8) \text {. }\end{array}$ \\
\hline $\begin{array}{l}\text { Transport Infra- } \\
\text { structure (World } \\
\text { Bank, 2013) }\end{array}$ & $\begin{array}{l}\text { Coverage: Infrastructure needs in } \\
\text { transport (roads). Gaps: Operation } \\
\text { and maintenance expenditure for } \\
\text { infrastructure. Rail, airports and } \\
\text { ports. }\end{array}$ & $\begin{array}{l}\text { Comprehensive coverage of interventions: } 0 \\
\text { Clear identification of inputs to address overlaps/gaps with other sectors: } 1 \\
\text { Intervention-based needs assessment: } 1 \\
\text { Goal-based analysis: } 0 \\
\text { Peer-reviewed assessment: } 0 \\
\text { Inclusion of operating and capital expenditure: } 0 \\
\text { Disaggregation of results by low- and lower-middle-income countries: } 0 \\
\text { Consideration of investment needs for climate change adaptation and mitiga- } \\
\text { tion: } 0 \\
\text { Inclusion of results from dynamic economy-wide models: } 0 \\
\text { Suitability score (out of } \mathbf{1 0 ) :} \mathbf{2 . 2}\end{array}$ & $\$ 283(2012-2030)$ & $\begin{array}{l}\text { Operating expenditure is estimated based on as- } \\
\text { sumption that O\&M represent } 51.6 \% \text { of total } \\
\text { transport infrastructure costs (Foster and Briceño- } \\
\text { Garmendia, } 2010 \text {, fig. O.3), rebased to } \$ 2013 \text {, and } \\
\text { scaled down to LICs and LMICs on a per capita } \\
\text { basis to yield } \$ 361 \text { billion per year (LICs: } \$ 90 \text { bil- } \\
\text { lion, LMICs: } \$ 271 \text { billion). A } 20 \% \text { mark-up on } \\
\text { capex is included for climate change adaptation } \\
\text { (Stern, 2007), yielding a total of } \$ 434 \text { billion per } \\
\text { year (LICs: } \$ 108 \text { billion, LMICs: } \$ 326 \text { billion). }\end{array}$ \\
\hline $\begin{array}{l}\text { Telecom infra- } \\
\text { structure (World } \\
\text { Bank, 2013) }\end{array}$ & $\begin{array}{l}\text { Coverage: Infrastructure needs in } \\
\text { telecommunications (fixed lines } \\
\text { and mobile connection). Gaps: } \\
\text { Operation and maintenance ex- } \\
\text { penditure for infrastructure. }\end{array}$ & $\begin{array}{l}\text { Comprehensive coverage of interventions: } 0 \\
\text { Clear identification of inputs to address overlaps/gaps with other sectors: } 1 \\
\text { Intervention-based needs assessment: } 1 \\
\text { Goal-based analysis: } 0 \\
\text { Peer-reviewed assessment: } 0 \\
\text { Inclusion of operating and capital expenditure: } 0 \\
\text { Disaggregation of results by low- and lower-middle-income countries: } 0 \\
\text { Consideration of investment needs for climate change adaptation and mitiga- } \\
\text { tion: } 0 \\
\text { Inclusion of results from dynamic economy-wide models: } 0 \\
\text { Suitability score (out of } \mathbf{1 0 ) :} \mathbf{2 . 2}\end{array}$ & $\$ 238(2012-2030)$ & $\begin{array}{l}\text { Operating expenditure is estimated based on as- } \\
\text { sumption that O\&M represent } 22.2 \% \text { of total tele- } \\
\text { com infrastructure costs (Foster and Briceño-Gar- } \\
\text { mendia, 2010, fig. O.3), rebased to } \$ 2013 \text {, and } \\
\text { scaled down to LICs and LMICs on a per capita } \\
\text { basis to yield } \$ 189 \text { billion per year (LICs: } \$ 47 \text { bil- } \\
\text { lion, LMICs: } \$ 142 \text { billion). Incremental costs for } \\
\text { climate change adaptation and mitigation are as- } \\
\text { sumed to be minimal and not taken into account. }\end{array}$ \\
\hline
\end{tabular}


4 - Investment needs to achieve SDGs in low- and lower-middle-income countries

\begin{tabular}{|c|c|c|c|c|}
\hline $\begin{array}{l}\text { SDG Invest- } \\
\text { ment Area } \\
\text { (source) }\end{array}$ & Coverage and key gaps & Quality assessment & $\begin{array}{l}\text { Results reported } \\
\text { in study (Period } \\
\text { average in } \$ \text { bil- } \\
\text { lions p.a.) }\end{array}$ & Adjustments made in this chapter \\
\hline $\begin{array}{l}\text { Ecosystems and } \\
\text { Biodiversity } \\
\text { (CBD, 2012a) }\end{array}$ & $\begin{array}{l}\text { Coverage: Achieving a subset of } \\
\text { GEF-eligible activities of 'strategic } \\
\text { importance' to the Aichi Goals and } \\
\text { Targets. Gaps: Analysis focuses } \\
\text { only on sub-set of activities of } \\
\text { 'strategic importance.' }\end{array}$ & $\begin{array}{l}\text { Comprehensive coverage of interventions: } 0 \\
\text { Clear identification of inputs to address overlaps/gaps with other sectors: } 1 \\
\text { Intervention-based needs assessment: } 1 \\
\text { Goal-based analysis: } 1 \\
\text { Peer-reviewed assessment: } 0 \\
\text { Inclusion of operating and capital expenditure: } 1 \\
\text { Disaggregation of results by low- and lower-middle-income countries: } 0 \\
\text { Consideration of investment needs for climate change adaptation and mitiga- } \\
\text { tion: } 0 \\
\text { Inclusion of results from dynamic economy-wide models: } 0 \\
\text { Suitability score (out of } 10): 4.4\end{array}$ & $\begin{array}{l}\$ 18-48(2014- \\
2018)\end{array}$ & $\begin{array}{l}\text { Rebased to } \$ 2013 \text {. Investment needs are scaled } \\
\text { down to LICs and LMICs on a per capita basis, } \\
\text { yielding total investment needs of } \$ 21-28 \text { billion } \\
\text { per year ( } \$ 5-7 \text { billion in LICs, } \$ 16-21 \text { billion in } \\
\text { LMICs). In the absence of data, investment needs } \\
\text { for climate change adaptation and mitigation are } \\
\text { not taken into account. }\end{array}$ \\
\hline $\begin{array}{l}\text { Data (Espey et } \\
\text { al., 2015) }\end{array}$ & $\begin{array}{l}\text { Coverage: Production and dissem- } \\
\text { ination of data to monitor progress } \\
\text { towards operationalizing and } \\
\text { achieving the SDGs. Gaps: Data } \\
\text { literacy; communication; long-term } \\
\text { programs of modernization of data } \\
\text { systems. }\end{array}$ & $\begin{array}{l}\text { Comprehensive coverage of interventions: } 1 \\
\text { Clear identification of inputs to address overlaps/gaps with other sectors: } 1 \\
\text { Intervention-based needs assessment: } 1 \\
\text { Goal-based analysis: } 1 \\
\text { Peer-reviewed assessment: } 0 \\
\text { Inclusion of operating and capital expenditure: } 1 \\
\text { Disaggregation of results by low- and lower-middle-income countries: } 1 \\
\text { Consideration of investment needs for climate change adaptation and mitiga- } \\
\text { tion: n/a } \\
\text { Inclusion of results from dynamic economy-wide models: } 0 \\
\text { Suitability score (out of } 10): \mathbf{7 . 5}\end{array}$ & $\$ 0.5(2016-2030)$ & $\begin{array}{l}\text { Estimates scaled down on a per capita basis to } \\
\text { LICs ( } \$ 0.3 \text { billion) and LMICs ( } \$ 0.1 \text { billion) for total } \\
\text { investment needs of } \$ \mathbf{0 . 4} \text { billion per year. }\end{array}$ \\
\hline $\begin{array}{l}\text { Humanitarian } \\
\text { work (N/A) }\end{array}$ & & $\begin{array}{l}\text { No long-term needs assessments are available for humanitarian work. See text } \\
\text { for how annual investment needs might be approximated. }\end{array}$ & & See text. \\
\hline
\end{tabular}


On metrics and financing for the Sustainable Development Goals

This chapter is based on the paper: Sachs J.D, G. Schmidt-Traub. 2017, "Global Fund lessons for Sustainable Development Goals," Science 356(6333), 32-33. (Corresponding author: Guido Schmidt-Traub) 


\section{Chapter 5}

Lessons from the Global Fund to Fight HIV/AIDS, Tuberculosis and Malaria for the Sustainable Development Goals 
On metrics and financing for the Sustainable Development Goals

The Global Fund to Fight AIDS, Tuberculosis and Malaria ('Global Fund') was established in 2001 and has since evolved into the largest multilateral funding mechanism for the control of the three infectious diseases. Third party assessments consistently rate the performance of the Global Fund as superior to most other multilateral financing mechanisms. This chapter considers the design of the Global Fund with particular attention to eight design principles that differentiate the institution from other financing mechanisms. We examine the performance of the Global Fund in light of the expectations put forward in 2001 and revisit major arguments against the Global Fund using evidence from the peer-reviewed and policy literature. The chapter then explores the relevance of the Global Fund's funding model for achieving the new Sustainable Development Goals (SDGs). We find that the eight design principles enabled the Global Fund to play a central role in advancing public health science and in scaling up proven interventions against the three infectious diseases in developing countries. It has evolved in light of lessons learnt and to address weaknesses in its operation. Based on its track record the Global Fund could become the major financing mechanism for health system strengthening, and its experience serves as a template for scaling up targeted interventions in other SDG areas. 


\subsection{Transparency and independence underpin successes ${ }^{5}$}

The Global Fund to Fight AIDS, Tuberculosis (TB), and Malaria was launched in 2001 in the context of the AIDS pandemic and the Millennium Development Goals (MDGs). Thanks in large part to key design principles (DPs), the Global Fund public-private partnership has played a major role in advancing public health science and in scaling up and strengthening evidence-based public health efforts in developing countries. As world leaders prepare to advance international development finance at the July 2017 Group of 20 (G20) Summit, we suggest the Global Fund as a template for funding research, development, and scale-up of interventions in both health and non-health areas of the Sustainable Development Goals (SDGs), which replaced MDGs in 2016.

As of 2000, there was little effort or consensus for deployment and scale-up of proven interventions against HIVIAIDS, TB, or malaria. Spending by affected countries was a fraction of the need; international aid for health was inadequate; and there was little implementation research on how to scale up complex health programs. Amid calls for increased action, United Nations Secretary-General Kofi Annan called in 2001 for creation of the Global Fund. Leaders of the Group of Seven (G7) countries endorsed the Global Fund; the first round of proposals was approved in April 2002; and first disbursements were made in January 2003.

Between 2003 and 2015, the Global Fund had disbursed $\$ 35$ billion, accounting for $16.4 \%$ of international funding for HIVIAIDS, $44.5 \%$ for TB, and $81.4 \%$ for malaria bed nets (IHME, 2016). By the end of 2014, Global Fund-supported programs had provided 8.1 million people with antiretroviral drugs (ARVs), distributed 548 million bed nets, treated 515 million people with artemisinin-based combination therapy, and treated 13.1 million people for TB (GFATM, 2015a).

\subsection{Eight design principles}

Eight DPs (GFATM, 2001) have made the Global Fund an innovative financing institution:

1. Country-led. Until 2010, countries submitted proposals outlining their financing needs rather than applying for funds allocated ex ante for each country. Since 2010, they apply for ex ante country allocations and modest incentive funding.

2. Multi-stakeholder. Each country submits proposals through its Country Coordination Mechanism (CCM), comprising representatives from government, civil society, business, development partners, and persons living with the diseases.

3. Independent, transparent, technical review, and evaluation. Proposals are reviewed by an independent Technical Review Panel (TRP) of scientific experts from public health and other disciplines. The Global Fund's independent Technical Evaluation Reference Group (TERG) and Office of the Inspector General (OIG) conduct audits and reviews of the Global Fund and its impact. Reports by the OIG, TRP, and TERG are made public, as are the Board's reactions, which is unique among bi- and multilateral financing institutions (HLIRP, 2011).

4. Political independence. The Governing Board-a body of donors, recipients, experts, civil society, and businesses-considers programs recommended by the TRP as a bloc to avoid political interference targeting individual countries.

\footnotetext{
${ }^{5}$ Annex 5 provides additional background information that was considered as part of the peer review of the paper on which this chapter is based. It provides additional references that could not be included in the published Science paper.
} 
5. Needs-based pooled financing. Donors pool resources into a single self-governing fund without earmarking and are expected to meet financing needs of Board-approved proposals through periodic replenishments.

6. Funding is for disease-specific programs but is implemented in broader health systems.

7. Performance-based funding. Continued funding is conditional on successful program execution and independent audits.

8. Financing only. As a financing instrument, rather than an implementing agency, the Global Fund relies on technical partners (e.g., World Health Organization (WHO) and UN Program on HIV/ AIDS) for technical advice or implementation support to countries.

The 2008 financial crisis led to a Global Fund funding crisis and the New Funding Model based on ex ante country allocations. The shift from 'demand-led' to 'allocationbased' funding brought the Global Fund more in line with practices at the World Bank and other donors. This has reduced allocations to some countries and slowed down financial scale up that had been under way until 2010 (IHME, 2016), a worrisome weakening of a central pillar of Global Fund success.

\subsection{Initial doubts, lessons learnt}

In light of calls for new global funds for SDGs, we revisit five early arguments against the Global Fund, and link subsequent evidence to key DPs.

First, it was widely deemed difficult or impossible to scale up proven but complex health interventions in resource-poor settings, and there was strong opposition to financing them publicly. In fact, the Global Fund generated large volumes of high-quality, country-led proposals recommended for funding by the TRP, particularly from poor countries. All TRP-recommended proposals were fully funded. Countries thus learned that rigorous programs would be funded, which spurred increased high-quality demand for funding [DPs 1, 4, and 5]. Global Fund-supported programs have performed well in complex operating environments of fragile countries (Bornemisza et al., 2010). Evaluations by the TERG supported implementation research by technical partners to fill knowledge gaps, such as the design of outcome and impact criteria in malaria-control programs (Nahlen and Low-Beer, 2007). Assessments of proposals by the TRP identified gaps in available interventions, such as rapid diagnostic tests for malaria [DP 3]. The Global Fund contributed to economies of scale, learning by doing, and price reductions (Zelman et al., 2014), which made free mass distribution of key commodities affordable. On the basis of Global Fund-funded implementation research, free mass distribution became the WHO standard for some commodities (e.g., ARVs) (WHO, 2007a) [DP 8].

Second, some feared that CCMs were externally driven and that they and the technical review process would undermine country ownership. Initial experiences were mixed, but CCM performance improved (HLIRP, 2011). Civil society involvement has improved the design and performance of many programs (Bridge et al., 2016) [DP 2]. The Global Fund's ability to disburse funds to a range of CCM-approved stakeholders (national and local governments, civil society organizations, international organizations, and businesses), combined with independent evaluation, has accelerated innovation and propagation of best practice (Jamison et al., 2013). 
Third, many argued that the 'vertical' focus of the Global Fund on specific diseases would undermine broader health systems strengthening (HSS) and distort health spending. But Global Fund-supported programs have been credited with contributing to HSS, although disease programs need to be better integrated into health systems. Global Fund funding does not appear to have reduced domestic or international spending on health systems (Macro International, 2009; Samb et al., 2009; iERG, 2014). Additional resources are needed to accelerate HSS (iERG, 2014; GFATM, 2015a) [DP $6]$.

Fourth, some argued that the health sector did not need a new financing institution and resisted calls for pooled international financing. Yet no multilateral institution was providing large-scale performance-based funding for disease control to a wide range of government and nongovernment parties. Today, most major donors contribute without earmarking, despite the preference of parliaments and governments for bilateral channels. The Global Fund, shown by independent evaluations to provide good 'value for money' (Macro International, 2009; HLIRP, 2011), has mobilized large volumes of additional aid for health [DP 5].

Fifth, many were concerned that the Global Fund's light-touch business model and reliance on external audits and technical partners would prove an easy target for corruption and poorly designed programs. In response, the Global Fund expanded staff in the mid-2000s to ensure better program oversight. In 2011, OIG flagged misuse of funds in several programs; less than $0.4 \%$ of resources had been misused (Brown and Griekspoor, 2013). The Global Fund recouped a large share of the loss and tightened corruption controls. Performance-based funding and low tolerance for corruption appear to improve programs, (e.g. Bass (2005)) [DP 7]. Transparency of funding decisions, disbursements, reporting, and evaluations contributes to success (HLIRP, 2011).

After initial difficulties (Macro International, 2009; HLIRP, 2011), the Global Fund improved cooperation with technical partners by promoting implementation research and assisting countries in preparing, implementing, and monitoring Global Fund-supported programs. In contrast to other financing mechanisms, the Global Fund makes all grantrelated data and audits public (PublishWhatYouFund, 2016). It works with partners to improve the quality and availability of data for disease and implementation metrics [DP 8].

In addition to successes, the Global Fund has experienced challenges (HLIRP, 2011). Countries and independent evaluations point to high transaction costs in accessing funding and a high reliance on consultants for proposal preparation and reporting. The Global Fund needs improved oversight of program implementation. This should include more explicit reporting on outcomes beyond the number of services provided, and transparent benchmarking to ensure effective use of resources and encourage performance-based funding. In addition to evaluations by Global Fund bodies and donors, monitoring by independent civil society organizations can play a role. The Global Fund should improve access to evaluation reports and country reporting and performance metrics. If the Global Fund remains committed to transparency and independent evaluations, these and other challenges can be addressed.

\subsection{Funds for achieving the SDGs}

The Global Fund should serve as the primary funding vehicle to implement strategies to break the three epidemics (90-90-90 Strategy for HIVI AIDS, the Global Plan to End TB, Global Malaria Action Plan) and to achieve SDG Target 3.3. The fact that the 
Global Fund was able to meet its $\$ 13$ billion target for the 2017-2019 replenishment is testament to broad support and perceptions of its success. Far greater resources will be required to fully implement recommended strategies against these diseases (GFATM, 2015a), so donors should expand their support.

The Global Fund should broaden its business model to increase investments and implementation research for HSS (SDG Target 3.8), reducing preventable deaths (Targets 3.1 and 3.2), access to sexual and reproductive health services (Target 3.7), responsiveness to disease outbreaks such as Ebola (Target 3.d), and tackling noncommunicable diseases (Target 3.4). As the main pooled financing vehicle for health systems and SDG health priorities, the Global Fund should work with Gavi, the vaccine alliance; World Bank; and others.

The Global Fund model can help meet investment needs in non-health SDG areas where proven interventions need to be scaled up with the help of public (co-)financing: e.g., smallholder farming (SDG 2), improved nutrition (SDG2 and SDG3), education (SDG 4), water supply and sanitation (SDG 6), and distributed rural electrification (SDG 7). The Global Fund model is not suited for investment areas, such as large-scale infrastructure, that require more market- and project-based financing solutions.

The recently announced Global Emergency Education Fund could be a step in the right direction. Together with the underfunded Global Partnership for Education, they should transform into a Global Fund for Education. Similarly, lessons from the Global Fund can inform the work and resource mobilization of existing multilateral financing institutions, e.g., the Green Climate Fund for climate change adaptation and mitigation, the Global Environment Facility for biodiversity and ecosystem management, and the International Fund for Agricultural Development.

\section{Acknowledgements}

The authors thank two anonymous reviewers for valuable comments. We are grateful for financial support from the Swedish International Development Cooperation Agency (Sida) 


\section{Annex 5. Supplementary material}

This Annex provides supplementary material that was shared with the editors of Science and the anonymous referees during the peer review of the manuscript. The material was subsequently removed from the manuscript to keep the length of the article in line with Science standards.

\section{A5.1. Context for the creation of the Global Fund}

As of the year 2000, the HIVIAIDS epidemic was raging uncontrolled, especially in subSaharan Africa. Anti-retroviral medicines (ARVs) had been in wide use in high-income countries since the mid-1990s. Yet in the poorer countries, and notably throughout Africa, ARVs were almost completely unavailable and unaffordable (Schwartländer et al., 2006). Malaria mortality and morbidity was surging despite new and effective treatment and control interventions, including artemisinin-based combination therapies (ACTs) and long-lasting insecticide-treated bed nets (LLINs) (Roll Back Malaria Partnership, 2008; HLIRP, 2011). In the case of TB, Directly-Observed Therapy ShortCourse (DOTS) was an established treatment regimen, and new treatments were becoming available against multi-drug resistant TB. Yet TB morbidity and mortality was rising because these proven treatments were not being scaled up (Stop TB Partnership, 2000).

In sum, little effort was being made to scale up the deployment of proven life-saving interventions despite the catastrophic human and economic toll exacted by the three diseases. Worse, there was no consensus on deploying these interventions. For example, the World Bank's proposals for scaling up the response to HIVIAIDS in poor countries did not mention ARVs as an option in 2000 (Binswanger, 2000). The spending by affected countries was a fraction of the true need, international aid for health was inadequate, and there was little implementation research on how to scale up complex health programs (CMH, 2001; UN Millennium Project, 2005).

In this context, activists, doctors, scientists, and political leaders called for greatly increased action against the major pandemics (Behrman, 2008). Médecins Sans Frontières, Partners in Health and others demonstrated the efficacy of antiretroviral treatment in low-income settings, albeit at very small scale (Farmer et al., 2001). A group of Harvard University faculty issued an influential statement calling for the scale-up of antiretroviral treatment in poor countries (Adams et al., 2001). One of us (Sachs) issued a call at the 2000 Durban AIDS Summit for a new 'global fund' to fight AIDS (Sachs, 2000), and led the WHO Commission on Macroeconomics and Health (20001), which recommended a large increase in aid for health, including for HIVIAIDS, TB, and malaria $(\mathrm{CMH}, 2001)$. The newly established Gates Foundation offered the possibility of substantial new funding for public health initiatives and supported effective advocacy in support of scaling-up.

Following UN Secretary-General Kofi Annan's call for the creation of the Global Fund in early 2001 (United Nations, 2001), US President George W. Bush and other G7 leaders endorsed the Global Fund, and it became operational in January 2002. The first round of country funding proposals was approved in April 2002, and first disbursements were made in January 2003. 


\subsubsection{A5.2. Global Fund grant cycle}

Figure 3 describes the grant cycle for the Global Fund. Countries form Country Coordination Mechanisms (CCM) comprising representatives from the government (typically the ministry of health), non-governmental organizations including faith-based organizations, academic institutions, business, affected communities, and development partners. The CCM may contract consultants to assist with the preparation of the proposals, but each proposal must be signed off by the CCM ensuring adequate voice for non-government representatives.

Proposals are then submitted to the Global Fund where the secretariat screens them for completeness and technical eligibility. It also confirms whether the CCM operates according to Global Fund standards. Screened proposals are reviewed by the TRP, which rates proposals on a scale of 1-4 (1, recommended for funding; 2 , recommended subject to technical revisions; 3 , not recommended, but encouraged to re-submit an improved proposal during a later round; 4, not recommended). The Board of the Global Fund then considers the TRP recommendations in toto, i.e. it can only approve all proposals as recommended by the TRP (possibly with across-the-board adjustments in funding volumes) or it can reject the entire set. This helps avoid political considerations interfering in the decisions of the Board. To this date, the Board has always followed the recommendations of the TRP in full, and all approved proposals have been fully funded.

On the basis of the approved proposals, the secretariat negotiates and signs grant agreements in line with the decisions of the Board. Following signature of the agreement, the Secretariat then instructs the Global Fund trustee (a function currently assumed by the World Bank) to disburse payments. Payments are made to the Principal Recipient (PR) and Sub-Recipient identified by the CCM as being best placed to implement the grant agreement. Any type of organization vetted and approved by the $\mathrm{CCM}$ can act as PR, including government ministries and agencies, international organizations (e.g. UNDP has frequently acted as PR in the Democratic Republic of Congo), civil society organizations (e.g. Médecins sans Frontières runs TB treatment programs in Somalia), or businesses. The flexible choice of PRs and sub-PRs has allowed the Global Fund to operate effectively in a broad range of different operating environments. It has also fostered innovation in program design and delivery (van Kerkhoff and Szlezák, 2010).

Finally, each grant is audited by an independent Local Fund Agent (LFA), such as major accounting firms (e.g. KPMG or PWC) or international organizations (e.g. UNOPS). The LFAs verify the transparent use of resources in accordance with the grant agreement and the results obtained. Their reports are taken into consideration by the secretariat before disbursing subsequent tranches of funding. 
Figure 3 | Global Fund grant cycle

Source: (Katz et al., 2010)

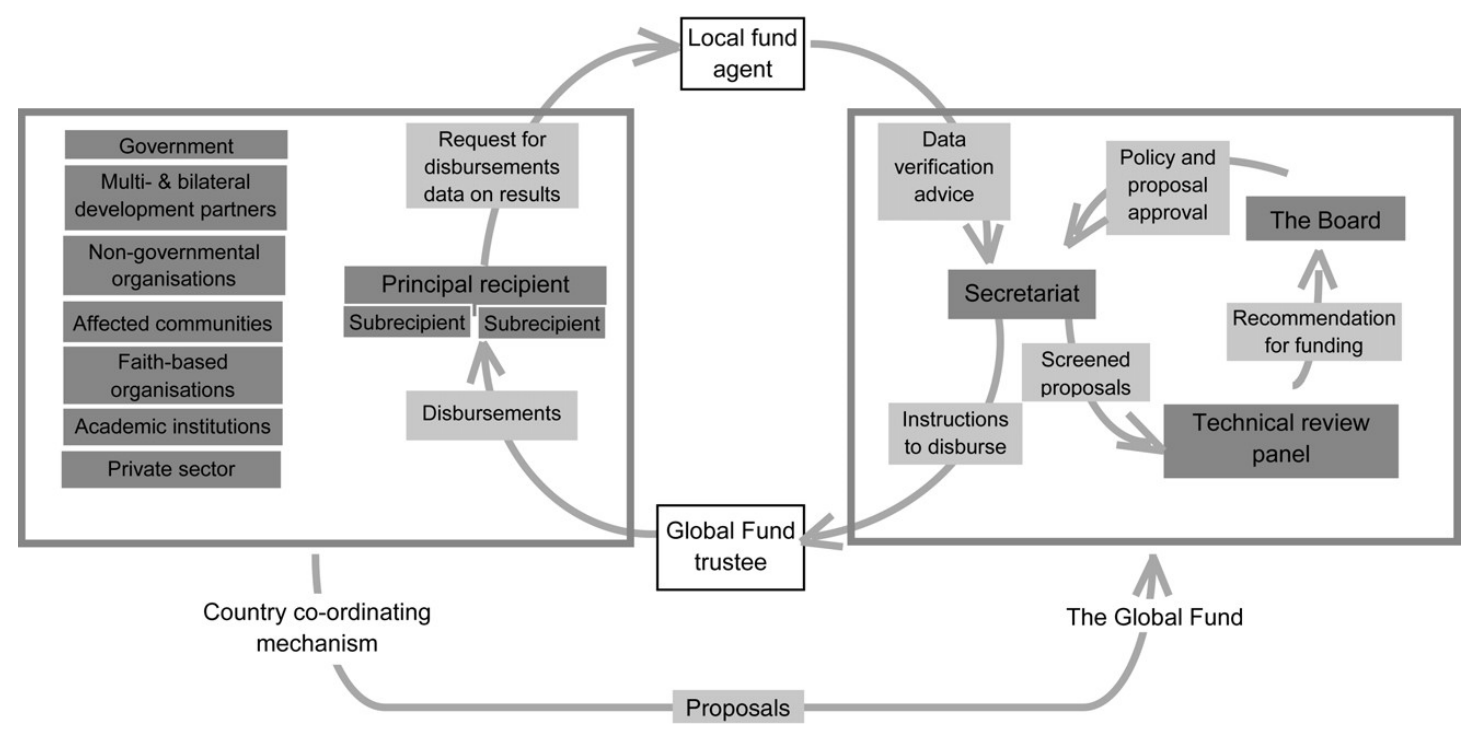

For additional information on how the Global Fund operates in practice see the guides prepared by Aidspan (Aidspan, 2017).

\section{A5.3. Significance of Global Fund for financing health goals}

The Global Fund made its first grant disbursement in January 2013. It quickly rose to become the dominant financing mechanisms for malaria and TB, accounting for $41,6 \%$ and $44.5 \%$, respectively, of all development assistance for these diseases. In HIVIAIDS it became the second largest individual funding channel after the bilateral US program PEPFAR (Table 28).

Table 28 | Largest channels for Development Assistance for Health 2003-2015

Table compares by disease total DAH for ten largest channels from 2003-2015 since the Global Fund made its first disbursement in January 2003. Calculations performed by author based on IHME data (IHME, 2016). DAH - Development Assistance for Health. NGO - nongovernmental organization. WHO - World Health Organization. BMGF - Bill and Melinda Gates Foundation. IDA - International Development Association (World Bank). Gavi - Global Alliance for Vaccine Immunization Initiative. NIH - National Institute of Health. IBRD - International Bank for Reconstruction and Development (World Bank).

\begin{tabular}{|c|c|c|c|c|c|c|c|c|c|c|c|c|}
\hline & \multicolumn{3}{|c|}{ DAH total } & \multicolumn{3}{|c|}{ DAH for HIVIAIDS } & \multicolumn{3}{|c|}{ DAH for TB } & \multicolumn{3}{|c|}{ DAH for malaria } \\
\hline Rank & Channel & \begin{tabular}{|c} 
Funding \\
(2014 \\
US\$ \\
billion)
\end{tabular} & Share & Channel & \begin{tabular}{|c} 
Funding \\
(2014 \\
US\$ \\
billion)
\end{tabular} & Share & Channel & \begin{tabular}{|c} 
Funding \\
(2014 \\
US\$ \\
billion)
\end{tabular} & Share & Channel & \begin{tabular}{|c|} 
Funding \\
(2014 \\
US\$ \\
billion)
\end{tabular} & Share \\
\hline 1 & bilateral USA & 71.59 & $17.6 \%$ & bilateral USA & 42.03 & $37.1 \%$ & Global Fund & 5.85 & $44.5 \%$ & Global Fund & 9.59 & $41.6 \%$ \\
\hline 2 & NGO & 68.44 & $16.9 \%$ & NGO & 23.24 & $20.5 \%$ & BMGF & 1.47 & $11.2 \%$ & NGO & 4.45 & $19.3 \%$ \\
\hline 3 & Global Fund & 34.64 & $8.5 \%$ & Global Fund & 18.54 & $16.4 \%$ & WHO & 1.37 & $10.4 \%$ & bilateral USA & 2.16 & $9.4 \%$ \\
\hline 4 & WHO & 30.92 & $7.6 \%$ & Int. NGO & 5.22 & $4.6 \%$ & NGO & 1.20 & $9.2 \%$ & BMGF & 2.00 & $8.7 \%$ \\
\hline 5 & Int. NGO & 24.89 & $6.1 \%$ & $\mathrm{NIH}$ & 4.28 & $3.8 \%$ & Int. NGO & 0.85 & $6.4 \%$ & WHO & 1.21 & $5.2 \%$ \\
\hline 6 & BMGF & 16.49 & $4.1 \%$ & UNAIDS & 3.52 & $3.1 \%$ & bilateral USA & 0.65 & $5.0 \%$ & bilateral UK & 1.15 & $5.0 \%$ \\
\hline 7 & UNICEF & 14.68 & $3.6 \%$ & bilateral UK & 2.76 & $2.4 \%$ & IDA & 0.42 & $3.2 \%$ & Int. NGO & 0.75 & $3.3 \%$ \\
\hline 8 & bilateral UK & 14.29 & $3.5 \%$ & BMGF & 2.69 & $2.4 \%$ & bilateral UK & 0.30 & $2.3 \%$ & IDA & 0.66 & $2.9 \%$ \\
\hline 9 & IDA & 13.69 & $3.4 \%$ & WHO & 1.45 & $1.3 \%$ & bilat. Canada & 0.29 & $2.2 \%$ & UNICEF & 0.26 & $1.1 \%$ \\
\hline 10 & Gavi & 13.64 & $3.4 \%$ & IDA & 1.31 & $1.2 \%$ & IBRD & 0.19 & $1.5 \%$ & bilat. Canada & 0.19 & $0.8 \%$ \\
\hline Total & & 406.03 & $100.0 \%$ & & 113.19 & $100.0 \%$ & & 13.15 & $100.0 \%$ & & 23.06 & $100.0 \%$ \\
\hline Top3 & & 174.67 & $43.0 \%$ & & 83.81 & $74.0 \%$ & & 8.69 & $66.1 \%$ & & 16.20 & $70.3 \%$ \\
\hline Top5 & & 230.47 & $56.8 \%$ & & 93.31 & $82.4 \%$ & & 10.74 & $81.7 \%$ & & 19.41 & $84.2 \%$ \\
\hline Top10 & & 303.26 & $74.7 \%$ & & 105.04 & $92.8 \%$ & & 12.60 & $95.8 \%$ & & 22.43 & $97.3 \%$ \\
\hline
\end{tabular}




\section{A5.4. Initial doubts, lessons learnt}

The head of USAID declared in 2001 that it would be impossible to provide ARVs in Africa because Africans could not tell time (The Boston Globe, 2001). Some argued that the interventions were too costly; others predicted that free distribution (e.g. of LLINs) would be abused (Easterly, 2006).

Instead, the TRP helped identify gaps in available health interventions and then drove down the cost of solutions, such as rapid-diagnostic tests for malaria (Zhao et al., 2012) [DP 3]. The Global Fund contributed significantly to economies of scale, learning by doing, and price reductions for key commodities, such as LLINs (Zelman et al., 2014) and ARVs (Stover et al., 2011). This made free mass distribution of key commodities more affordable, and the evidence suggests it has worked well (WHO, 2007b; Piot et al., 2009; Dizon-Ross et al., 2015; WHO, 2016c). Free mass distribution became the official WHO standard for ARVs in the early 2000s and for LLINs in 2007, based in particular on implementation research from a Global Fund-funded program in Kenya (WHO, 2007a) [DP 8].

Initial experiences with the CCMs were mixed (McKinsey, 2005), but their performance improved over time (Atun and Kazatchkine, 2009; HLIRP, 2011; Kageni et al., 2015). China is an interesting example. The country's first two HIVIAIDS proposals were rejected by the TRP, inter alia for not including harm reduction programs [DPs 3, 4]. For Round 3 the country adopted international best practice and mobilized stakeholders through the CCM. It now credits its success in tackling HIVIAIDS on the Global Fundfunded programs and the CCM (Wang et al., 2014; Minghui et al., 2015). The involvement of civil society organizations in the disease control efforts has improved the design and performance of many Global Fund-funded programs, including by expanding and strengthening harm reduction (Atun and Kazatchkine, 2010; Bridge et al., 2016) [DP 2]. The Global Fund's ability to disburse funds to a range of CCM-approved stakeholders (national and local governments, civil society organizations, international organizations, and businesses), combined with independent program evaluation, has accelerated innovation and propagation of best practice (van Kerkhoff and Szlezák, 2010; Jamison et al., 2013).

From 2003-2015 Global Fund-supported programs have accounted for 95.0\% and $31.3 \%$ of funding for malaria- and HIVIAIDS-related Health Systems Strengthening (HSS), respectively (IHME, 2016).

The Global Fund significantly expanded its headcount in the mid-2000s to ensure better program oversight. Concerns rose to the fore in 2011 after the independent IOG flagged misuse of funds in several programs, but less than $0.4 \%$ of resources had been misused (Brown and Griekspoor, 2013; HLIRP, 2011). The corresponding programs were terminated, the Global Fund managed to recoup a large share of the loss, and corruption control measures were tightened further. The evidence suggests overall that the Global Fund ensures transparent and sound use of resources (Macro International, 2009; French Ministry of Foreign Affairs, 2013). Independent reviews find evidence for performance-based funding by the Global Fund, but with substantial discretion exercised by the Global Fund secretariat (Radelet and Siddiqi, 2007; Katz et al., 2010; Fan et al., 2013). Low tolerance for corruption and performance-based funding appear to improve program performance (Bass, 2005) [DP 7]. Throughout the high transparency of funding decisions, disbursements, reporting, and evaluations has contributed to the success of the Global Fund and on-going improvements of its operations and effectiveness (HLIRP, 2011). 
The Global Fund has improved its cooperation with RBM (Simon et al., 2013), Stop TB (McKinsey, 2008), and UNAIDS (Poate et al., 2009) with each technical partner promoting implementation research and assisting countries in preparing, implementing, and monitoring Global Fund-supported programs. Clear operating modalities specify the division of labor to avoid conflicts of interest between technical advisory and implementation functions, (WHO, 2015a, p. 64).

The fight against the three major diseases remains unfinished and requires increased international resources (Jamison et al., 2013; Roll Back Malaria Partnership, 2008; UNAIDS, 2014; Stop TB Partnership, 2015). The Global Fund should serve as the primary funding vehicle to implement the strategies to break the three epidemics: the 90-90-90 Strategy for HIVIAIDS (UNAIDS, 2014), the Global Plan to End TB (Stop TB Partnership, 2015), and the Global Malaria Action Plan (Roll Back Malaria Partnership, 2008). It is encouraging that donors committed close to $\$ 13$ billion at the September 2016 replenishment round hosted by the Government of Canada. The fact that the Global Fund was able to meet its declared funding target is testament to broad support for the Global Fund and widely held perceptions of its success, as the replenishment took place in a challenging funding environment marked by deep cuts to European aid budgets in response to the migration crisis and uncertainty following the Brexit vote in the UK. Yet far greater resources will be required to fully implement the recommended public health strategies against the three diseases (GFATM, 2015a), so donors should certainly expand their support to the Global Fund in coming years. 


\section{On metrics and financing for the Sustainable Development Goals}

A revised and expanded version of this chapter has been accepted for publication as: Schmidt-Traub, G. (forthcoming), "The role of the Technical Review Panel of the Global Fund to Fight HIVIAIDS, Tuberculosis and Malaria: An analysis of grant recommendations," Health Policy and Planning. 


\section{Chapter 6}

The role of the Technical Review Panel of the Global Fund: An analysis of grant recommendations

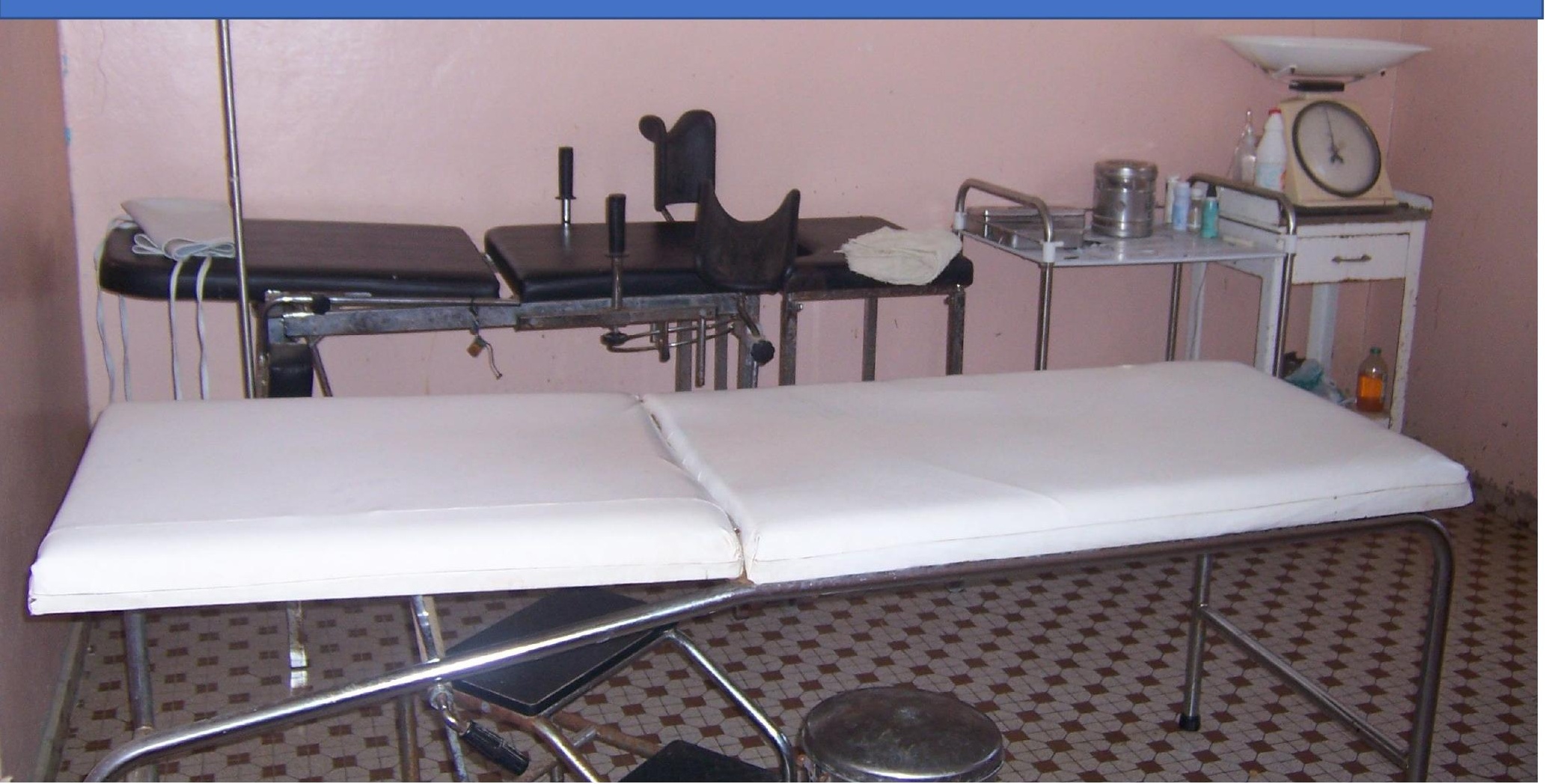


The independent Technical Review Panel (TRP) of the Global Fund to Fight HIVIAIDS, Tuberculosis and Malaria is a unique mechanism to review funding proposals and to provide recommendations on their funding. The functioning and performance of the TRP have received little attention in the scientific literature. We aimed to identify predictors for TRP recommendations, whether these were in line with the Global Fund's ambition to give priority to countries most in need, and whether they correlated with grant performance. To this end, we combined data on proposals, TRP recommendations, and grant implementation during the rounds-based mechanism (2002-2010) with country characteristics. Ordered logistic and OLS regressions were used to identify predictors for per-capita funding requests, TRP recommendations, Global Fund funding to each country, and grant performance ratings. We tested for financial suppression of large funding proposals and whether fragile or English-speaking countries performed differently from other countries. We found that funding requests and TRP recommendations were consistent with disease burden, but independent of other country characteristics. Countries with larger populations requested less funding per capita, but the TRP did not exercise financial suppression. Proposals from fragile countries were equally likely to be recommended as proposals from other countries, and resulting grants performed equally well except for lower performance of HIVIAIDS grants. English-speaking countries obtained more funding for TB and malaria than other countries. In conclusion, the independent TRP acted in line with the guiding principles of the Global Fund to direct funding to countries most in need without ex ante country allocation. The Global Fund promoted learning on how to design and implement largescale programs in fragile and non-fragile countries. Other pooled financing mechanisms should consider adopting TRP operating principles to generate high-quality demand, to promote learning, and to direct resources to countries most in need. 


\subsection{Introduction}

Since its establishment in 2001 the Global Fund to Fight AIDS, Tuberculosis and Malaria (henceforth the Global Fund) has become the dominant external funding channel for the three diseases except for HIVIAIDS where it is second to the US President's Emergency Plan for AIDS Relief (PEPFAR) (IHME, 2016). In late 2016 the Global Fund mobilized some $\$ 13$ billion at a time when international aid budgets were under severe pressure owing to the refugee crisis in Europe and macroeconomic pressures on donor countries.

The success of the Global Fund in generating quality demand for health financing and in mobilizing substantial volumes of development assistance for health (DAH) has been attributed to its unique design principles (Sachs and Schmidt-Traub, 2017). These include a focus on "national ownership and respect [for] country-led formulation and implementation"; the evaluation of "proposals through independent review processes based on the most appropriate scientific and technical standards"; giving "due priority to the most affected countries"; and "linking resources to the achievement of clear, measurable and sustainable results" (GFATM, 2001). The Global Alliance for Vaccines and Immunization (Gavi) applies similar design principles and has been equally successful in increasing quality DAH demand for vaccines.

The independent Technical Review Panel (TRP), which reviews proposals and makes funding recommendations to the Board, is a key innovation of the Global Fund (Sachs and Schmidt-Traub, 2017). Among all institutions reviewed in the 2016 UK Multilateral Development Review (DFID, 2016), Gavi is the only other mechanism that operates an independent review panel. The Global Fund Board has always followed TRP funding recommendations, and every Board-approved country program has been fully funded. So, although the Board took final funding decisions, they have been determined by the TRP.

During the rounds-based funding mechanism (2002-2010) the TRP was asked to issue recommendations without ex ante country allocations based on the technical merit of each proposal. Recommendations were made by consensus on a scale of 1-4 (1, recommended; 2, recommended subject to clarifications; 3 , not recommended but strongly encouraged to re-submit; 4 , not recommended). During the first ten rounds $42 \%$ of proposals were rated 1 or 2 . To maintain the technical independence of the evaluation process and to avoid interference with political considerations relating to individual countries, the Global Fund Board voted en bloc on the entire set of TRP recommendations. Countries could appeal against funding decisions, and for most rounds some appeals were granted (GFATM, 2016a).

After 2011 the Global Fund introduced the New Funding Model under which countries can apply for pre-agreed volumes of funding with an additional volume of modest 'incentive funding' (GFATM, 2013). TRP recommendations now prioritize interventions within a pre-agreed resource envelope. This diminished the discretion of countries and the TRP to set funding volumes, so we limit our analysis to the rounds-based mechanism.

Several studies have investigated the performance of Global Fund grants (Lu et al., 2006; Radelet and Siddiqi, 2007; Macro International, 2009; Katz et al., 2010; Fan et al., 2013), including in fragile countries (Bornemisza et al., 2010; Patel et al., 2015), and their impact on health systems (Atun et al., 2011; Car et al., 2012; iERG, 2014; Samb et al., 2009). There has been no systematic assessment of the role of the TRP. This study addresses three questions that are central to understanding the demandbased funding model of the Global Fund: 
1. Which factors explained the volume of funding requested by countries for each disease? We studied whether countries' funding requests were in line with funding needs as defined by income and disease burden or whether there was evidence of financial suppression of large grants (i.e. lower ratings of proposals requesting higher total volumes of funding after controlling for other explanatory variables). We also tested for differences in the ratings of proposals from fragile countries and non-English speaking countries since the latter had been reported (Kerouedan, 2010; French Ministry of Foreign Affairs, 2013) to face greater difficulties in accessing funding.

2. Which factors explained TRP ratings of grant proposals? We checked whether ratings were consistent with the Global Fund's ambition to give priority to countries most in need. We tested for evidence of financial suppression of proposals by the TRP and improvements in the quality of disease proposals (learning).

3. Were TRP recommendations and other proposal characteristics a good predictor of successful implementation of Global Fund grants, and was there evidence for changes in proposal quality over time?

The success of scaling-up health investments despite initial concerns about absorptive capacity (Lu et al., 2006) stands in contrast to other investment priorities. Health systems, non-communicable diseases, environmental health, and non-health-related areas have not experienced a similar scaling up of aid (de Jongh et al., 2014; IHME, 2016). They have also seen a lower acceleration of progress under the Millennium Development Goals (McArthur and Rasmussen, 2017). Achieving the recently adopted Sustainable Development Goals (SDGs) will require large increases in domestic and external funding for health systems, non-communicable diseases, environmental health (Jamison et al., 2013), education, and other areas (Schmidt-Traub, 2015). With Gavi and the Global Fund routinely rated among the best performing financing mechanisms (DFID, 2016) we also considered whether the performance of the TRP may hold lessons for scaling up funding in other areas.

\subsection{Materials and methods}

A new dataset was generated combining data on funding proposals submitted to the TRP provided by the Global Fund secretariat; TRP recommendations and Board decisions; grants and their performance; and country characteristics consistent with predictors used by previous studies (Lu et al., 2006; Katz et al., 2010; Radelet and Siddiqi, 2007; Fan et al., 2013; Bowser et al., 2014). Proposals rated 1 or 2 by the TRP were manually matched with signed grants from the grants dataset using proposal parameters provided in both datasets, including the proposal name, disease component, country, funding volumes, and the year of submission. Summary statistics, data sources, and construction of dependent variables are described in the Annex. The accompanying Stata dataset and do-file allow for replication of the analysis.

Regression 1 (Table 29) tests the first question, by conducting a linear ordinary least squares (OLS) regression for each disease with combined TB-HIVIAIDS programs included under HIVIAIDS, consistent with earlier studies (Radelet and Siddiqi, 2007; Fan et al., 2013). The model was specified as Equation 10:

$$
\mathrm{y}_{\mathrm{i}}=\mathrm{X}_{\mathrm{i}} \beta_{\mathrm{i}}+\varepsilon_{\mathrm{i}}
$$

Where the dependent variable $y_{i}$ is the log of total per capita funding requested by disease i per Global Fund round, $X_{i}$ denotes the matrix of regressors including proposal 
and country characteristics as described in Table 29. $\beta_{\mathrm{i}}$ is the matrix of regression coefficients estimated in this OLS model, and $\varepsilon_{\mathrm{i}}$ is the disturbance term for each disease. Each regressor comprised data from the year preceding the respective Global Fund round since the TRP considered proposals on the basis of this information.

Regression 2 (Table 30): To address the second question and to investigate predictors of the TRP recommendations, we constructed an ordered logistic regression model regressing TRP ratings (1-4) on the same proposal and country characteristics, as in regression 1 . Note that lower TRP ratings denoted higher-quality proposals. For each disease $\mathrm{i}$, the probability $\mathrm{P}$ that the TRP rating $\mathrm{y}_{\mathrm{i}}$ took on the value $\mathrm{l}$ (ranging from $1-4)$ is denoted by Equation 11:

$$
P\left[y_{i}=l\right]=F\left(\alpha_{l}-X_{i} \beta_{i}\right)-F\left(\alpha_{l-1}-X_{i} \beta_{i}\right)
$$

where $\mathrm{F}$ is the logistic cumulative density function, $\alpha_{1}$ the threshold for the observed value $1, \alpha_{1-1}$ the threshold for the observed value $\mathrm{l}-1, \beta_{\mathrm{i}}$ the matrix of regression coefficients estimated (including intercept terms), and $X_{i}$ denotes the matrix of regressors described in Table 30.

Regression 3 (Table 31): To combine the effects of requested funding volumes per round (dependent variable in Regression 1) with approval rates (dependent variable in Regression 2) and the frequency with which a country applied for funding, Regression 3 (Table 31) used OLS and the same functional form as Regression 1 to assess predictors of total per capita Global Fund funding received by country and by disease during the rounds-based mechanism. The dependent variable $y_{\mathrm{i}}$ was defined as the $\mathrm{In}$ of total per capita funding received by disease $i$ during the rounds-based funding mechanism. The regressors $x_{i j}$ are described in Table 31. DAH from the Global Fund excluded DAH for the disease a regression focused on. Since we were interested in the effects over the full duration of the rounds-based mechanism, the independent variables were expressed as the mean over the period 2001-2010.

Regression 4 (Table 32): TRP reports (TRP, 2009) and earlier investigations (Lu et al., 2006; Radelet and Siddiqi, 2007; Katz et al., 2010) suggested that grant performance ratings were poorly specified and subject to significant discretion by the secretariat (Fan et al., 2013). In Regression 4 (Table 32) we did not attempt to resolve these issues and focused instead on investigating the relationship between TRP recommendations and the performance of approved grants and whether fragile or English-speaking countries performed significantly differently. An ordered logit model with the same functional form as for regression 2 was estimated for each disease $i$ the probability $P$ that the average Phase 1 performance rating $y_{i}$ took on the value I (ranging from 1-5 with lower values denoting stronger performance). Table 32 describes the regressors. In this way, we assessed whether TRP standards might have changed over time, particularly with repeat submissions of proposals.

Significance of regressors was established at $p<0.05$, and each model was subjected to stepwise backward elimination of non-significant predictors (Chatterjee and Hadi, 2015) to confirm robustness of predictors. Standard post-regression tests were conducted for data outliers, homoscedasticity, normality of residuals, and multi-collinearity of predictors (Annex 6).

\subsection{Results and discussion}

This session presents and discusses the results from the regressions. 
Regression 1 (Table 29): After controlling for other factors, higher disease prevalence was associated with higher requested funding volumes $(p<0.001)$. Population was negatively correlated $(p<0.001)$ with per capita funding requests, suggesting that large countries exercised financial suppression in Global Fund proposals. This is consistent with the broader aid literature (Younas, 2008; Bourguignon et al., 2009; Temple, 2010), but studies into DAH (Lu et al., 2010) have not controlled for population size. Coefficients for resubmitted proposals were not significant, so countries did not reduce funding requests following an initial rejection by the TRP. If countries believed the TRP exercised financial suppression they would reduce funding requests upon resubmission to increase the likelihood of a TRP recommendation. Proposals that followed the successful approval of a first grant to the country did not request significantly different funding volumes.

The year of the round was correlated with larger funding requests $(p<0.001)$ consistent with a scaling-up of program size over time. The coefficient was small, but since over this period the real cost of disease interventions, such as antiretroviral therapy (Stover et al., 2011) or malaria interventions (Zelman et al., 2014), fell sharply, the evidence suggests that countries designed their proposals around a substantial scaling-up of interventions.

GDP per capita and domestic health spending were not significantly associated with funding requests, and DAH from non-Global Fund donors was a significant predictor only for higher malaria funding requests. Global Fund DAH was significantly associated with higher funding requests for HIVIAIDS proposals. Countries with a stronger health system, as measured by the Universal Health Care (UHC) tracer, requested lower per capita funding for HIVIAIDS and malaria, consistent with a high unmet financing need for health system strengthening. In the case of TB, the relationship was reversed, possibly because strong health systems allowed for larger-scale programs.

Countries with lower government effectiveness requested more funding for HIVIAIDS and TB, but this did not translate into higher approved funding volumes (Regression 3 ). Countries with a higher score on the governance variable 'voice and accountability' requested more funding, but this effect was only significant for HIVIAIDS and TB after removing less significant predictors from the model (Annex 6). Control of corruption was not a significant predictor.

PEPFAR focus countries requested significantly more funding, suggesting that PEPFAR technical assistance for HIVIAIDS program design outweighed lower residual funding needs compared with non-PEPFAR countries. The dummies for fragile and English-speaking countries were not significant, except for a positive correlation of the English-speaking country dummy with malaria funding requests.

Regression 2 (Table 30): Few predictors were significant in explaining TRP ratings. Population was non-significant, and became significantly associated with better ratings after stepwise elimination of insignificant predictors (Annex 6). Funding proposals from countries with large populations did not receive poorer ratings, suggesting that the TRP did not exercise financial suppression.

After a first grant had been approved by the Global Fund, subsequent proposals from the same country became associated with better TRP ratings $(p<0.001)$. In addition, resubmitted proposals received better TRP ratings, but the association was not significant for TB proposals. This evidence is consistent with either a lowering of TRP standards for subsequent proposals or a learning effect leading to higher-quality proposals. Since average TRP ratings were positively associated with the year of the round (sig- 
nificant for malaria proposals after stepwise elimination), countries appear to have improved the quality of repeat submissions. Evidence from Regression 4 shows that dummies for resubmitted proposals or proposals following the approval of the first grant for the country were not significantly associated with grant performance ratings, which supports the interpretation that the TRP did not lower its standards for such proposals, and instead we see evidence of learning by countries.

Per capita GDP was not a significant predictor of TRP ratings except for HIVIAIDS proposals where income correlated with better TRP ratings $(p<0.05)$. Likewise, TRP ratings were not significantly associated with governance variables except for malaria grants where the coefficient for 'voice and accountability' was positive $(p<0.05)$.

There is no evidence that the TRP discriminated against fragile countries or that these countries had greater difficulties in submitting high-quality proposals. English-speaking countries were more likely to receive better TRP ratings for malaria grants $(p<0.05)$. The relationship was not significant for the other diseases. After stepwise elimination of insignificant predictors, PEPFAR countries were significantly associated with better proposal ratings, reinforcing the suggestion (Regression 1) that they received enhanced technical assistance in program design.

Domestic health spending and DAH were not significantly associated with TRP ratings, except for Global Fund DAH for TB, which correlated with worse TRP ratings. Regression 3 shows that this did not translate into a significant effect on overall grant volumes for TB. The UHC tracer, TB treatment success, and prevalence rates were not significant predictors of TRP ratings. On balance, TRP recommendations were not correlated with funding needs (disease prevalence) or other regressors. Instead the TRP appears to have assessed only the technical quality of the proposals, consistent with its mandate during the rounds-based mechanism.

Regression 3 (Table 31): Cumulative funding volumes were positively associated with disease prevalence for each disease $(p<0.05)$, suggesting that the TRP mechanism solicited and recommended high-quality funding requests from higher-burden countries. The association with per capita GDP was significantly negative for HIVIAIDS, and the negative coefficient became significant for TB stepwise elimination. On balance, the Global Fund directed higher per capita funding towards poorer countries.

Population size was negatively associated with overall funding volumes $(p<0.001)$. So, the self-suppression of funding requests from larger countries (Regression 1) resulted in lower funding volumes even though there was no evidence of financial suppression in TRP recommendations (Regression 2).

A stronger health system was associated with higher TB funding, consistent with the need for health systems to scale up TB interventions. Meanwhile, the association was negative for malaria grants $(p<0.001)$, possibly due to the dominant role played by the Global Fund in financing malaria bed nets, which could be distributed outside health systems (Hafner and Shiffman, 2013; de Jongh et al., 2014) and were most needed in poorer countries that tended to have weaker health systems.

Global Fund funding did not substitute domestic health expenditure. The associations with DAH and governance variables were also non-significant.

Fragile states were not significantly associated with per capita funding volumes, except for HIVIAIDS grants where they received significantly less funding. Considering evidence from the first two regressions, fragile countries submitted fewer proposals, but 
their proposals did not receive worse TRP ratings. This suggests greater scope for technical assistance to fragile countries to accelerate proposal design.

English-speaking countries received more funding $(p<0.05)$ for TB and malaria grants. The correlation was not significant for HIVIAIDS. This lends support to concerns (Kerouedan, 2010; French Ministry of Foreign Affairs, 2013) that non-English-speaking countries were less successful at attracting Global Fund funding. For malaria grants Regressions 1 and 2 show that English-speaking countries submitted larger proposals that were more likely to be approved by the TRP. In the case of TB, English-speaking countries submitted proposals more frequently. Global Fund funding for HIVIAIDS was complementary to PEPFAR funding since PEPFAR countries were positively associated with higher per capita funding volumes, reflecting both larger (Regression 1) and higher-quality (Regression 2) proposals.

Regression 4 (Table 32): Controlling for other factors, average Phase 1 performance ratings did not correlate significantly with TRP recommendations. A plausible explanation is that all proposals rated 2 were revised with guidance from the TRP and Global Fund secretariat, which increased their quality to a point where they matched or exceeded that of proposals rated 1 .

Grants resulting from resubmitted proposals or following the approval of an earlier grant did not perform significantly differently. This suggests that higher TRP ratings for such proposals (Regression 2) did not reflect lower TRP assessment standards but signified higher-quality proposals. This confirms evidence from other studies that the TRP encouraged and helped propagate significant learning on design and implementation of disease programs by countries (van Kerkhoff and Szlezák, 2010; Stover et al., 2011; Jamison et al., 2013; Bridge et al., 2016). For example, following two subsequent rejections of its HIVIAIDS proposals by the TRP, China reformed its approach to managing the disease by including international best practice on harm reduction. Similarly, TRP requirements to adhere to medical best practice by involving communities and people living with the diseases in program design and implementation, also had a deep impact on China's malaria control program (Wang et al., 2014; Minghui et al., 2015).

We found no evidence that grants to fragile countries performed significantly differently from grants to non-fragile countries. Using a smaller dataset, an earlier study (Bornemisza et al., 2010) found a small, negative correlation between grant performance and fragility. PEPFAR focus countries and English-speaking countries were not associated with significant differences in performance ratings. The latter suggests that the Global Fund overcame language barriers during grant implementation even though English-speaking countries obtained higher funding volumes for some diseases.

Limitations: Even though the R-squared are relatively high, the reported associations could also be due to factors not considered in the models. Global Fund practices (e.g. TRP procedures, evaluation standards, the role of the Technical Partners, modalities for Principal Recipients and Local Fund Agents) improved over time, and these effects may not have been picked up fully in the regression analyses. As discussed, data quality of grant performance ratings has been questioned, so Regression 4 could only establish the plausibility of consistent TRP assessment standards during the roundsbased mechanism. Historic data on intervention coverage during the rounds-based mechanism were too sparse to construct comprehensive models of Global Fund grant performance. 


\subsection{Conclusion}

During the rounds-based mechanism (2002-2010) per capita funding requests to the Global Fund were correlated with disease prevalence rates and they increased over time in the presence of sharp falls in the cost of interventions, consistent with a significant scaling-up of programs in fragile and non-fragile countries alike. The Global Fund promoted substantial learning and improvements in the quality of country proposals. It is likely that the TRP's transparent rating of proposals, the release of findings from each funding round, and the systematic review of lessons learnt with Technical Partners (e.g. WHO, UNAIDS, Stop TB, and Roll-Back Malaria) contributed to propagating knowledge on scaling up interventions across countries. The country-led funding model encouraged scaling-up since countries were not constrained in the volume of per capita funding they could request, and success in one country inspired others to follow.

The data do not support the conclusion of the 2011 review of Global Fund operations that "large countries put forward enormous proposals" (HLIRP, 2011). In fact, the opposite was the case. Per capita funding to and funding requests from countries with larger populations were financially suppressed, even though TRP recommendations were unaffected by population size. This finding challenges a central argument in support of the New Funding Model and suggests significant unmet funding needs, particularly in countries with larger populations. These lessons should be considered in designing and possibly expanding the Global Fund's incentive funding mechanisms.

Throughout the rounds-based mechanism, the TRP fulfilled its role: It recommended proposals without regard to funding volumes, population size, or other country characteristics typically associated with different aid volumes, such as governance, domestic health expenditure, and DAH. Global Fund funding went towards higher-burden countries with lower incomes, consistent with the guiding principles of the Global Fund.

The Global Fund, working with its Technical Partners, was effective at overcoming lower capacity to design and implement programs in poorer and/or fragile countries. Fragile countries requested funding volumes that were not significantly different from those requested by other countries; they were as likely to have their funding requests approved by the TRP; and their grants performed equally well except for HIVIAIDS grants. Indeed, the TRP flagged concerns about the low quality of technical assistance for HIVIAIDS grants (TRP, 2009), which might explain the differential performance of fragile countries here. Conversely, higher funding requests and better ratings of proposals from PEPFAR countries suggest that countries that receive greater support in strengthening and scaling-up their response to HIVIAIDS can attract more funding from the Global Fund. This in turn suggests the presence of large unmet funding needs in non-PEPFAR countries, which technical assistance might be able to convert into highquality proposals.

The Global Fund's success in operating across the full spectrum of country environments, including fragile states, sets an example for financing health systems and other investment priorities under the SDGs. For example, the education sector currently operates a separate pooled funding mechanism for countries in emergency situations. Given the need to lower transaction costs, and the continuous transition from fragile to non-fragile environments, it would appear preferable to operate one integrated pooled financing mechanism for education. Such a mechanism would promote learning and the propagation of operational lessons across all countries.

English-speaking countries obtained higher volumes of funding for malaria and TB. TRP reports frequently referred to quality problems with the translation of proposals 
and supporting documents into English. The Global Fund and other mechanisms must ensure that language does not become a barrier to accessing financing.

Taken together, the evidence demonstrates that a demand-based funding mechanism relying on independent technical review of proposals without ex-ante country allocations can generate needs-based funding allocations. It can stimulate quality demand even in poor and fragile countries contrary to widespread expectations in 2002 (Sachs and Schmidt-Traub, 2017). In short, the TRP has played the role it was expected to.

Pooled international funding mechanisms in other areas, such as the Global Environment Facility, the Green Climate Fund, the Global Partnership for Education, or the International Fund for Agricultural Development, should study the workings and performance of the TRP and may consider establishing similar procedures. Further work is needed to study how lessons from the TRP can be applied to other sectors and to understand how the role of the TRP has evolved under the New Funding Model. 
Table 29 | Predictors of requested funding volumes per round, by disease

Data are OLS regression coefficients (SE) (Regression 1). Sample is restricted to grant proposals from individual countries that were considered by the TRP during the rounds-based mechanism (2002-2010) and for which requested funding volumes are recorded. Sources and definitions of the variables are available in the appendix. GDP - gross domestic product. pc - per capita. 2014 cUS\$ - constant 2014 US\$. DAH - Development Assistance for Health. PEPFAR - President's Emergency Plan for AIDS Relief. ${ }^{*} p<0.05,{ }^{* *} p<0.01,{ }^{* * *}$ $p<0.001$.

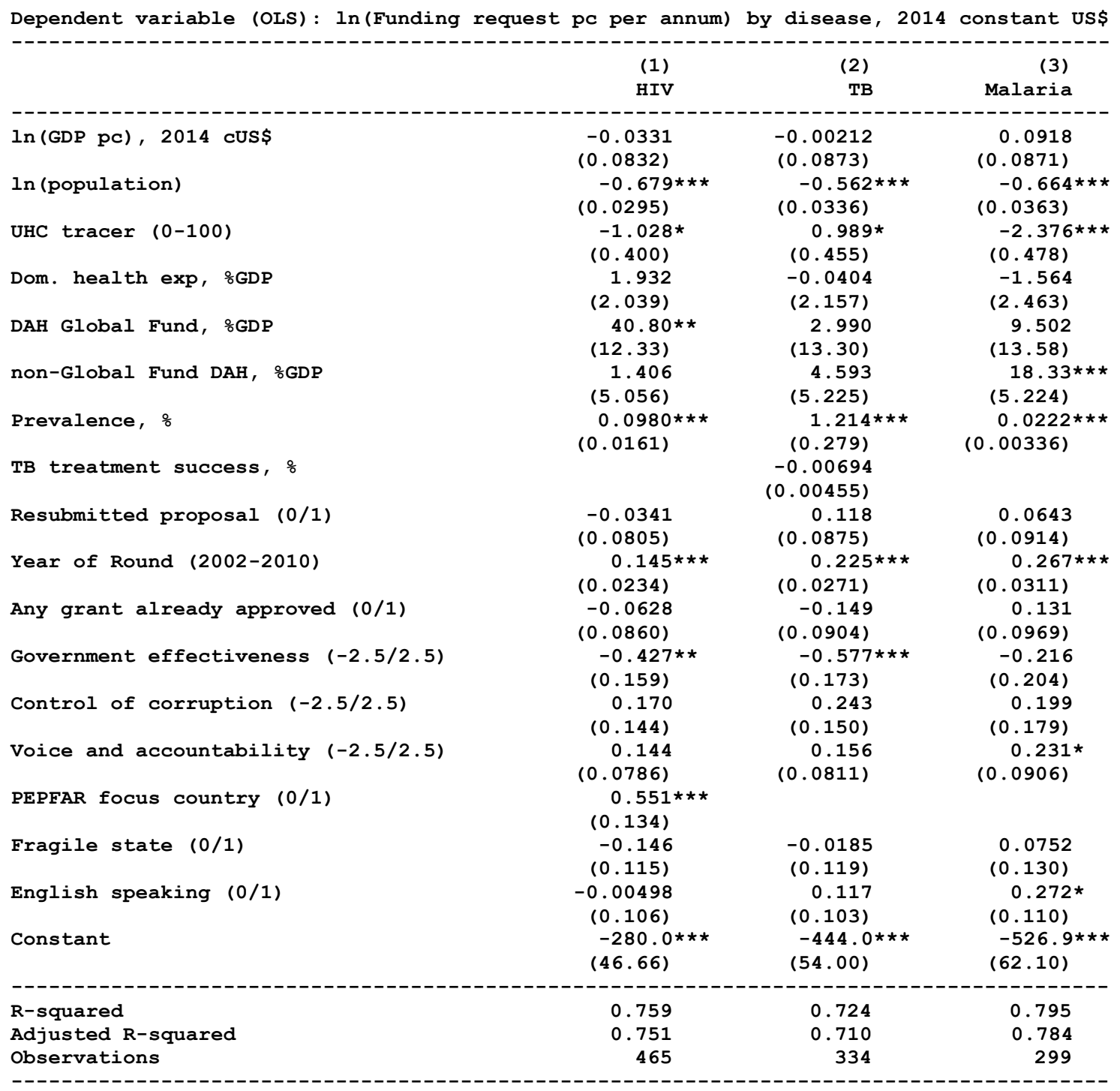


Table 30 | Predictors for TRP proposal ratings, by disease

Data are ordered logistic regression odds ratios (SE) without constant (Regression 2). Sample is restricted to grant proposals from individual countries that were considered by the TRP during the rounds-based mechanism (2002-2010). Sources and definitions of the variables are available in the appendix. GDP - gross domestic product. pc - per capita. 2014 cUS\$ constant 2014 US\$. DAH - Development Assistance for Health. UHC - Universal Health Coverage. PEPFAR - President's Emergency Plan for AIDS Relief. ${ }^{*} p<0.05,{ }^{* *} p<0.01$, $p<0.001$.

Dependent variable (ordered logit): TRP rating (1-4), odds ratios

\begin{tabular}{|c|c|c|c|}
\hline & $\begin{array}{l}\text { (1) } \\
\text { HIV }\end{array}$ & $\begin{array}{r}(2) \\
\text { TB }\end{array}$ & $\begin{array}{r}(3) \\
\text { Malaria }\end{array}$ \\
\hline $\ln (G D P$ pc), 2014 cUS\$ & $\begin{array}{l}-0.693 \star \\
(0.286)\end{array}$ & $\begin{array}{l}0.00717 \\
(0.314)\end{array}$ & $\begin{array}{r}0.634 \\
(0.383)\end{array}$ \\
\hline In (population) & $\begin{array}{l}0.0686 \\
(0.139)\end{array}$ & $\begin{array}{l}-0.0459 \\
(0.162)\end{array}$ & $\begin{array}{l}0.0985 \\
(0.231)\end{array}$ \\
\hline UHC tracer $(0-100)$ & $\begin{array}{r}0.424 \\
(1.368)\end{array}$ & $\begin{array}{l}-2.052 \\
(1.699)\end{array}$ & $\begin{array}{r}1.909 \\
(2.359)\end{array}$ \\
\hline Dom. health exp, GDP & $\begin{array}{r}6.495 \\
(7.157)\end{array}$ & $\begin{array}{r}3.522 \\
(8.530)\end{array}$ & $\begin{array}{l}-2.606 \\
(11.08)\end{array}$ \\
\hline DAH Global Fund, $\frac{\circ}{\circ} \mathrm{DP}$ & $\begin{array}{l}-8.358 \\
(45.47)\end{array}$ & $\begin{array}{l}96.96 * \\
(46.12)\end{array}$ & $\begin{array}{r}48.62 \\
(59.21)\end{array}$ \\
\hline non-Global Fund DAH, $\%$ GDP & $\begin{array}{l}-14.36 \\
(17.14)\end{array}$ & $\begin{array}{l}-15.88 \\
(20.10)\end{array}$ & $\begin{array}{r}23.55 \\
(24.11)\end{array}$ \\
\hline Prevalence, $\%$ & $\begin{array}{r}0.0488 \\
(0.0595)\end{array}$ & $\begin{array}{r}-1.407 \\
(1.068)\end{array}$ & $\begin{array}{r}0.0295 \\
(0.0164)\end{array}$ \\
\hline TB treatment success, $\frac{\circ}{0}$ & & $\begin{array}{r}0.0177 \\
(0.0164)\end{array}$ & \\
\hline In (board approved total pc), 2014 cUS\$ & $\begin{array}{l}-0.220 \\
(0.152)\end{array}$ & $(0.203)$ & $\begin{array}{l}-0.449 \\
(0.255)\end{array}$ \\
\hline Resubmitted proposal $(0 / 1)$ & $\begin{array}{l}-0.610 \star \\
(0.280)\end{array}$ & $\begin{array}{l}-0.479 \\
(0.335)\end{array}$ & $\begin{array}{l}-1.364 * \star \\
(0.468)\end{array}$ \\
\hline Year of Round (2002-2010) & $\begin{array}{l}0.253 * \star \\
(0.0856)\end{array}$ & $\begin{array}{l}0.242 \star \\
(0.116)\end{array}$ & $\begin{array}{r}0.187 \\
(0.164)\end{array}$ \\
\hline Any grant already approved $(0 / 1)$ & $\begin{array}{l}-7.338 \star \star \star \\
(0.774)\end{array}$ & $\begin{array}{l}-8.014 * \star * \\
(1.087)\end{array}$ & $\begin{array}{l}-8.743 * \star \star \\
(1.186)\end{array}$ \\
\hline Government effectiveness $(-2.5 / 2.5)$ & $\begin{array}{l}-0.399 \\
(0.538)\end{array}$ & $\begin{array}{r}0.129 \\
(0.693)\end{array}$ & $\begin{array}{l}-0.406 \\
(0.852)\end{array}$ \\
\hline Control of corruption $(-2.5 / 2.5)$ & $\begin{array}{r}0.0521 \\
(0.488)\end{array}$ & $\begin{array}{r}0.915 \\
(0.568)\end{array}$ & $\begin{array}{r}0.105 \\
(0.751)\end{array}$ \\
\hline Voice and accountability $(-2.5 / 2.5)$ & $\begin{array}{r}0.347 \\
(0.268)\end{array}$ & $\begin{array}{l}-0.121 \\
(0.326)\end{array}$ & $\begin{array}{l}0.792 * \\
(0.386)\end{array}$ \\
\hline PEPFAR focus country $(0 / 1)$ & $\begin{array}{r}0.647 \\
(0.482)\end{array}$ & & \\
\hline Fragile state $(0 / 1)$ & $\begin{array}{l}-0.171 \\
(0.395)\end{array}$ & $\begin{array}{r}0.147 \\
(0.434)\end{array}$ & $\begin{array}{r}0.894 \\
(0.572)\end{array}$ \\
\hline English speaking $(0 / 1)$ & $\begin{array}{r}0.307 \\
(0.369)\end{array}$ & $\begin{array}{l}0.0108 \\
(0.382)\end{array}$ & $\begin{array}{l}-1.270 * \\
(0.501)\end{array}$ \\
\hline $\begin{array}{l}\text { Pseudo R-squared } \\
\text { Observations }\end{array}$ & $\begin{array}{r}0.517 \\
464\end{array}$ & $\begin{array}{r}0.537 \\
334\end{array}$ & $\begin{array}{c}0.636 \\
299\end{array}$ \\
\hline
\end{tabular}


Table 31 | Predictors for total per capita Global Fund funding, by disease

Data are OLS regression coefficients (SE) (Regression 3). Sample is restricted to grants approved under the rounds-based mechanism (2002-2010). Sources and definitions of the variables are available in the appendix. GDP - gross domestic product. pc-per capita. 2014 cUS\$ - constant 2014 US\$. DAH - Development Assistance for Health. PEPFAR - President's Emergency Plan for AIDS Relief. ${ }^{*} p<0.05,{ }^{* *} p<0.01,{ }^{* * *} p<0.001$.

Dependent variable: In(total signed funding pc) rounds-based mechanism, 2014 const. US\$

\begin{tabular}{|c|c|c|c|}
\hline & $\begin{array}{l}(1) \\
\text { HIV }\end{array}$ & $\begin{array}{r}(2) \\
\text { TB }\end{array}$ & $\begin{array}{r}\text { (3) } \\
\text { Malaria }\end{array}$ \\
\hline 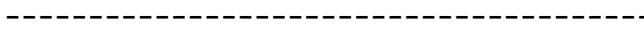 & -----------1 & ------------1 & ----------- \\
\hline $\ln (a v$ GDP pc), 2014 cUS\$ & $\begin{array}{l}-0.4521 \text { * } \\
(0.1949)\end{array}$ & $\begin{array}{c}-0.3205 \\
(0.1815)\end{array}$ & $\begin{array}{c}-0.0518 \\
(0.1579)\end{array}$ \\
\hline In (av population) & $\begin{array}{l}-0.5502 \star \star \star \\
(0.0726)\end{array}$ & $\begin{array}{l}-0.4719 * \star \star \\
(0.0646)\end{array}$ & $\begin{array}{l}-0.5633 * \star \star \\
(0.0652)\end{array}$ \\
\hline av UHC tracer $(0-100)$ & $\begin{array}{r}0.5223 \\
(0.9995)\end{array}$ & $\begin{array}{l}2.3904 \text { * } \\
(1.0042)\end{array}$ & $\begin{array}{l}-3.2203 * \star \star \\
(0.9206)\end{array}$ \\
\hline av Dom. health exp, \%GDP & $\begin{array}{r}4.2055 \\
(5.2168)\end{array}$ & $\begin{array}{c}-0.7168 \\
(4.3294)\end{array}$ & $\begin{array}{c}-2.5929 \\
(4.6890)\end{array}$ \\
\hline Global Fund DAH residual, ஃGDP & $\begin{array}{r}82.632 \\
(72.511)\end{array}$ & $\begin{array}{r}20.052 \\
(32.666)\end{array}$ & $\begin{array}{r}37.910 \\
(42.666)\end{array}$ \\
\hline av non-Global Fund DAH, $\frac{\circ}{\circ} \mathrm{GDP}$ & $\begin{array}{r}-2.064 \\
(16.417)\end{array}$ & $\begin{array}{r}-8.261 \\
(11.756)\end{array}$ & $\begin{array}{c}19.398 \\
(12.190)\end{array}$ \\
\hline av Prevalence, \% & $\begin{array}{l}0.1768 * \\
(0.0787)\end{array}$ & $\begin{array}{c}1.8773 * \star \star \\
(0.5438)\end{array}$ & $\begin{array}{l}0.0440 * \star \star \\
(0.0104)\end{array}$ \\
\hline av TB treatment success, $\frac{\circ}{0}$ & & $\begin{array}{r}0.0031 \\
(0.0098)\end{array}$ & \\
\hline av Control of corruption $(-2.5 / 2.5)$ & $\begin{array}{c}-0.1758 \\
(0.3847)\end{array}$ & $\begin{array}{c}-0.2831 \\
(0.3333)\end{array}$ & $\begin{array}{c}-0.1246 \\
(0.3844)\end{array}$ \\
\hline av Government effectiveness $(-2.5 / 2.5)$ & $\begin{array}{l}-0.4891 \\
(0.4394)\end{array}$ & $\begin{array}{l}-0.2498 \\
(0.3682)\end{array}$ & $\begin{array}{r}0.2472 \\
(0.4718)\end{array}$ \\
\hline av Voice and accountability $(-2.5 / 2.5)$ & $\begin{array}{c}0.1614 \\
(0.1871)\end{array}$ & $\begin{array}{r}0.0563 \\
(0.1682)\end{array}$ & $\begin{array}{c}-0.1397 \\
(0.1820)\end{array}$ \\
\hline av PEPFAR focus country $(0-1)$ & $\begin{array}{l}1.3285 * \star \\
(0.4978)\end{array}$ & & \\
\hline Fragile state $(0-1)$ & $\begin{array}{l}-0.7395 * \\
(0.3237)\end{array}$ & $\begin{array}{c}-0.3502 \\
(0.2749)\end{array}$ & $\begin{array}{c}-0.2222 \\
(0.2711)\end{array}$ \\
\hline English speaking $(0 / 1)$ & $\begin{array}{r}0.1425 \\
(0.2880)\end{array}$ & $\begin{array}{l}0.5373^{\star} \\
(0.2119)\end{array}$ & $\begin{array}{c}0.4968 * \\
(0.2141)\end{array}$ \\
\hline Constant & $\begin{array}{l}12.6355 * \star \star \\
(2.0848)\end{array}$ & $\begin{array}{l}8.3533 * \star \star \\
(1.8610)\end{array}$ & $\begin{array}{l}11.5731 \star \star \star \star \\
(1.7591)\end{array}$ \\
\hline R-squared & 0.653 & 0.632 & 0.824 \\
\hline Adjusted R-squared & 0.607 & 0.575 & 0.788 \\
\hline Observations & 111 & 97 & 72 \\
\hline
\end{tabular}


Table 32 | Predictors for Phase 1 performance rating, by disease

Data are ordered logistic regression odds ratios (SE) without constant (Regression 4). Sample is restricted to grant proposals from individual countries that were approved during the rounds-based mechanism (20022010). Sources and definitions of the variables are available in the appendix. GDP - gross domestic product. pc - per capita. 2014 cUS\$ - constant 2014 US\$. TRP - Technical Review Panel. DAH - Development Assistance for Health. UHC - Universal Health Coverage. LFA - Local Fund Agent. PWC - PriceWaterHouse Coopers. STPH - Swiss Tropical and Public Health Institute. UNOPS - United Nations Operations and Project Services. PR - Principal Recipient. CSO - Civil Society Organization. PEPFAR - President's Emergency Plan for AIDS Relief. ${ }^{*} p<0.05,{ }^{* *} p<0.01,{ }^{* *} p<0.001$.

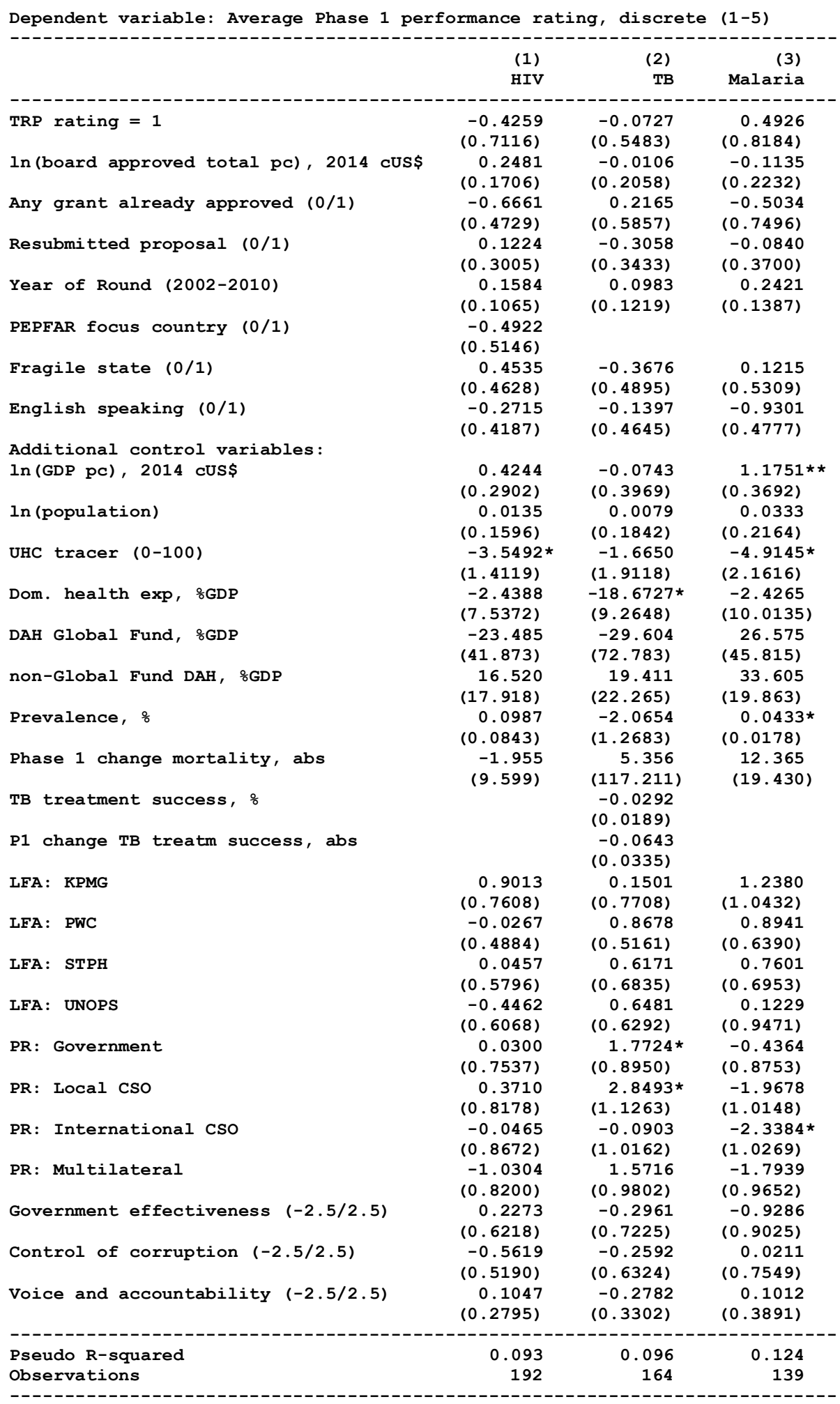




\section{Acknowledgements}

The Swedish International Development Cooperation Agency (Sida) provided funding for this study. Jeffrey Sachs and Ekko van lerland advised on the design of the study. Christoph Benn, Lucie Blok, Sofia Cordero, David Durand-Delacre, George Gotsadze, Ilze Kalnina, Jeroen Klomp, and Katerina Teksoz have provided valuable comments. David Durand-Delacre and Murad Hirji assisted with data collection and preparation. The views expressed are those of the author and do not represent those of Sida or of the Sustainable Development Solutions Network (SDSN).

\section{Role of the funding source}

The funder had no role in study design, data collection, data analysis, data interpretation, or writing of the report. The corresponding author had full access to all the data in the study and had final responsibility for the decision to submit for publication. 


\section{Annex 6. Supplementary material}

\section{A6.1. Construction of the dataset and regression variables}

The Global Fund secretariat provided the author with a dataset on country proposals submitted to the TRP throughout the rounds-based funding mechanism (2002-2011) of the Global Fund. This dataset contained information on all proposals that were submitted to the TRP, including country coverage, disease component, requested funding volume, adjustments to the funding volume proposed by the TRP, TRP ratings, date of submission, round, and whether the proposal was submitted by a CCM. The dataset also specified whether a proposal was ultimately approved and signed by the Global Fund. Each proposal was identified by a unique proposal ID. With the exception of Round 1 and a few parameters for other rounds, these data were also publicly available through the Board decisions published on the Global Fund's website in the form of non-machine-readable PDFs.

As a first step, we cleaned up the dataset by harmonizing the spelling of country names, country codes, and regions using World Bank classifications (World Bank, 2016a). All multi-country and regional proposals were removed for the regression analyses since they could not be matched with country characteristics.

Six countries (Barbados, Lebanon, Lithuania, Poland, Seychelles, and Vanuatu) each submitted a proposal for HIVIAIDS during the first three rounds. The proposals were not recommended by the TRP, and the countries did not re-apply for Global Fund funding in subsequent rounds. We excluded these countries from the sample.

Where necessary, funding requests were converted from Euro into constant 2014 US\$ using World Bank deflators and exchange rates. For some proposals, the requested volume of funding was missing in the database. Where possible this information was obtained from published TRP reports to the Global Fund Board (GFATM, 2016b). In some rounds (e.g. Round 8 ) the TRP introduced rating sub-categories $2 \mathrm{~A}$ and $2 \mathrm{~B}$ to some grants. Since these sub-categories were not used consistently throughout all rounds and since all recommended Round 8 grants were funded, we combined all rating sub-categories into an overall rating of 2 .

The dataset did not contain information linking proposals approved by the Board with the unique ID for each grant signed by the Global Fund. We therefore manually matched every proposal rated 1 or 2 by the TRP with the grant IDs in the Global Fund database using proposal parameters provided in both datasets, including the proposal name, disease component, country, funding volumes, and the year of submission. Each Global Fund grant ID provides unambiguous information on the country name, disease category, funding round, and the sequence of the signed grants, which facilitated the manual matching with TRP proposals. Out of 992 proposals recommended by the TRP 8 did not lead to a signed grant.

The following Global Fund data on grants were downloaded on 7 December 2016 from the Global Fund website: implementation periods (GFATM, 2016c), grant agreement progress updates,(GFATM, 2016d) grant agreements,(GFATM, 2016e) and contact organizations (GFATM, 2016f).

Table 33 provides the codebook for all regression variables. The full dataset is available online.

The Global Fund split some proposals into several grant agreements, e.g. to allow for different Principal Recipients. In such cases the corresponding proposal ID was 
matched to each grant ID, and where necessary duplicate proposals were removed from the regression analyses. We also generated variables for the year of each round and the corresponding deadline for proposals using information provided in Global Fund Board Decisions (GFATM, 2016b).

Under Global Fund rules, countries could resubmit revisions of proposals that had been rated 3 by the TRP. To identify resubmitted proposals, we generated a dummy variable that assigned a value of 1 to every proposal that was submitted within two rounds of a previous proposal rated 3 of the same disease component. Another dummy variable tracked whether the country already had a Global Fund grant approved in any disease category at the time of submitting the new proposal.

Wang et al. (2016) disease data were retained for regression analysis owing to broadest coverage, consistency, and frequency. It provided prevalence and incidence data in 5-year intervals. The gaps were closed through a geometric sequence extrapolation. For example, 2001 prevalence data were estimated as prev2002 = prev2000 * (prev2005/2010)^(2/5). TB prevalence data from WHO (WHO, 2016d) were available annually, so we used this data in lieu of interpolating IHME data on TB prevalence.

The annual fragile state dummy was created by combining the World Bank list of fragile states (World Bank, 2016b) and the Fragile States Index (Fund for Peace, 2016). The dummy assigns 1 to every country that is either on the World Bank list or the Fragile States Index. No data were available prior to 2006, so the fragile state dummy for the years 2001-2005 was set equal to 2006 data.

Funding requests, GDP, and population were transformed into natural logarithms to track the proportional effect of these scale variables. We expressed domestic health expenditure and DAH data in percent of GDP to avoid multicollinearity with per capita GDP.

For time-series OLS and logistic regressions on country and proposal characteristics (regressions 1, 2, 4), we used the country characteristics from the year preceding the TRP review of the proposal. For example, all proposals considered in round 3 in November 2003 were regressed on GDP per capita and disease data from 2002. For OLS regression 3 of total Global Funding approved during the round-based mechanism (2002-2010) we generated a variable comprising the mean over the period 2002-2010.

\section{A6.2. Regression models}

Regression 1: The matrix of regressors $x_{i j}$ is described in Table 37, and Table 34 provides summary statistics.

Regression 2: The matrix of regressors $x_{i j}$ is described in Table 38, and Table 34 provides summary statistics.

Regression 3: The regressors $x_{i j}$ are described in Table 39, and summary statistics are presented in Table 35.

Regression 4: Table 40 describes the regressors, and Table 36 presents summary statistics.

\section{A6.3. Post-regression diagnostics}

Key findings from the post-regression diagnostics are summarized below. 
- Unusual and influential data (outliers): Extreme outlier values were identified using a matrix of plots of each bivariate combination of regression variables as well as added variable plots (also known as partial regression plots). We then assessed the potential impact of outliers on the regressions by removing them from the estimation. The following observations appeared problematic:

- Domestic health expenditure (\%GDP): Moldova's health expenditure for proposal (ID 2072) was 2 SD higher than next highest. Other observations for Moldova were within the range of other countries. Removing this value did not affect the results, but improved the linearity of the results as well as the normality of the residuals. This observation was therefore removed from the sample.

- Non-Global Fund DAH (\%GDP): Data for three proposals from Liberia (IDs 1001, 1002, 1040) were more than 3 SD higher than the secondhighest country, as well as other observations for the same country. Removing these three observations increased the significance of the predictor and improved normality of the residuals. We therefore removed these data points.

- Multicollinearity: We estimated the variance inflation factor (vif) for the variables in each regression equation and checked the pairwise correlation coefficients. No regression equation exhibited high levels of multicollinearity.

- Normality of residuals: The Shapiro-Wil test suggested normally distributed residuals for all regressions with the exception of the HIVIAIDS regression 1 and regressions 3 for which we could not reject the hypothesis of non-normal residuals. Visual inspection of kernel density and normal probability plots suggested that the deviation from normality was modest and affected only the center of the distribution.

- Homoscedasticity: According to the Breusch-Pagan test we could not reject the hypothesis at heteroscedasticity at $p<0.05$ for the regressions 1 and regression 2 for HIVIAIDS. Visual inspection of a plot of residuals against the fitted line (rvfplot) suggested that the problems of heteroscedasticity were modest.

- Linearity: Linearity was tested using augmented partial residual plots, also known as augmented component-plus-residual (ACR) plots. No major deviations from the linearity hypothesis were observed with the exception of some of the above-mentioned variables comprising major outliers.

\section{A6.4. Robustness of coefficients}

To assess the robustness of the models and predictors, each model was subjected to stepwise backward elimination of non-significant predictors (Chatterjee and Hadi, 2015). For each regression, we present up to three sets of regression results: (1) the full model; (2) a reduced version following the step-by-step elimination of regression variable with $p$-value greater than 0.20 while retaining predictors describing proposal characteristics regardless of their significance, and (3) the fully reduced model following the step-by-step elimination of all regressions with $p$-value greater than 0.20 . Results are described below for Regression 1 (Table 37), Regression 2 (Table 38), Regression 3 (Table 39), and Regression 4 (Table 40). 
Table 33 | Codebook for regression variables

* If no source is listed then variable was generated by the author from other information in the dataset.

\begin{tabular}{|c|c|c|c|}
\hline Variable label & Regression & Comment & Source* \\
\hline Any grant already approved $(0 / 1)$ & $1,2,4$ & & \\
\hline average Control of corruption (-2.5/2.5) & 3 & Average 2001-2010 & World Bank (2016e) \\
\hline average DAH Global Fund (\%GDP) & 3 & Average 2001-2010 & IHME (2016) \\
\hline $\begin{array}{l}\text { average Domestic health expenditure } \\
(\% \text { GDP })\end{array}$ & 3 & Average 2001-2010 & WHO (2016e) \\
\hline average Fragile country $(0-1)$ & 3 & Average $2001-2010$ & $\begin{array}{l}\text { World Bank (2016b), } \\
\text { Fund for Peace (2016) }\end{array}$ \\
\hline $\begin{array}{l}\text { average Government effectiveness (- } \\
2.5 / 2.5)\end{array}$ & 3 & & World Bank (2016e) \\
\hline average Non-Global Fund DAH (\% GDP) & 3 & Average 2001-2010 & IHME (2016) \\
\hline average PEPFAR focus country $(0-1)$ & 3 & Average 2001-2010 & PEPFAR (2016) \\
\hline $\begin{array}{l}\text { average Phase } 1 \text { performance rating, } \\
\text { discrete }(1-5)^{\circ}\end{array}$ & 4 & Dependent variable & GFATM (2016d) \\
\hline average Prevalence (per 100) & 3 & Average 2001-2010 & $\begin{array}{l}\text { Wang et al. (2016), } \\
\text { WHO (2016d) }\end{array}$ \\
\hline average TB treatment success, $\%$ & 3 & Average 2001-2010 & WHO (2016d) \\
\hline $\begin{array}{l}\text { average Universal Health Coverage (UHC) } \\
\text { tracer }(0-100)\end{array}$ & 3 & Average $2001-2010$ & GBD (2016) \\
\hline average Voice and accountability $(-2.5 / 2.5)$ & 3 & Average $2001-2010$ & World Bank (2016e) \\
\hline $\begin{array}{l}\text { Change in mortality during Phase } 1 \text {, } \\
\text { absolute }\end{array}$ & 4 & & \\
\hline $\begin{array}{l}\text { Change TB treatment success during Phase } \\
1, \%\end{array}$ & 4 & & \\
\hline Control of corruption (-2.5/2.5) & $1,2,4$ & Data from year before round & World Bank (2016e) \\
\hline DAH Global Fund (\%GDP) & $1,2,4$ & Data from year before round & IHME (2016) \\
\hline Domestic health expenditure (\%GDP) & $1,2,4$ & Data from year before round & WHO (2016e) \\
\hline English speaking country $(0 / 1)$ & $1,2,3,4$ & & CIA (2016) \\
\hline Fragile country $(0 / 1)$ & $1,2,4$ & Data from year before round & $\begin{array}{l}\text { World Bank (2016b), } \\
\text { Fund for Peace (2016) }\end{array}$ \\
\hline Global Fund DAH residual (\%GDP) & 3 & Average $2001-2010$ & IHME (2016) \\
\hline Government effectiveness (-2.5/2.5) & $1,2,4$ & Data from year before round & World Bank (2016e) \\
\hline $\begin{array}{l}\text { In(average GDP per capita), constant } 2014 \\
\text { US\$ }\end{array}$ & 3 & Average $2001-2010$ & World Bank (2016b) \\
\hline In(average population) & 3 & Average 2001-2010 & World Bank (2016b) \\
\hline $\begin{array}{l}\text { In(Board approved total per capita), constant } \\
2014 \text { US\$ }\end{array}$ & 2,4 & & GFATM (2016e) \\
\hline $\begin{array}{l}\text { In(Funding request per capita p.a.), constant } \\
2014 \text { US } \$^{\circ}\end{array}$ & 1 & Dependent variable & \\
\hline In(GDP per capita), constant 2014 US\$ & $1,2,4$ & Data from year before round & World Bank (2016b) \\
\hline $\ln ($ Population) & $1,2,4$ & Data from year before round & World Bank (2016b) \\
\hline $\begin{array}{l}\text { In(total signed funding per capita) rounds- } \\
\text { based mechanism, constant } 2014 \text { US } \$^{\circ}\end{array}$ & 3 & Dependent variable & \\
\hline $\begin{array}{l}\text { Local Fund Agent (PWC, KPMG, STPH, } \\
\text { UNOPS, other) }\end{array}$ & 4 & Factor variable & GFATM (2016e) \\
\hline Non-Global Fund DAH (\%GDP) & $1,2,4$ & Data from year before round & IHME (2016) \\
\hline PEPFAR focus country $(0 / 1)$ & $1,2,4$ & Data from year before round & PEPFAR (2016) \\
\hline Prevalence (per 100) & $1,2,4$ & Data from year before round & $\begin{array}{l}\text { Wang et al. (2016), } \\
\text { WHO (2016d) }\end{array}$ \\
\hline $\begin{array}{l}\text { Principal Recipient Type (Government, local } \\
\text { CSO, int. CSO, multilateral agency, other) }\end{array}$ & 4 & Factor variable & GFATM $(2016 e)$ \\
\hline Proposal approved $^{\circ}$ & 2 & Dependent variable & \\
\hline Proposal rated 1 by TRP & 4 & & \\
\hline Resubmitted proposal (0/1) & $1,2,4$ & & \\
\hline TB treatment success, $\%$ & $1,2,4$ & Data from year before round & WHO (2016d) \\
\hline TRP rating $(1-4)^{\circ}$ & 2 & Dependent variable & \\
\hline $\begin{array}{l}\text { Universal Health Coverage (UHC) tracer ( } 0 \text { - } \\
100)\end{array}$ & $1,2,4$ & Data from year before round & GBD (2016) \\
\hline Voice and accountability $(-2.5 / 2.5)$ & $1,2,4$ & Data from year before round & World Bank (2016e) \\
\hline Year of Round (2002-2010) & $1,2,4$ & & GFATM (2016b) \\
\hline
\end{tabular}


Table 34 | Summary statistics for Regression 1 and Regression 2, by disease

Data are number of observations (count), mean, and standard deviation (SD). Sample is restricted to grant proposals from individual countries that were considered by the TRP during the rounds-based mechanism (2002-2010). Sources and definitions of the variables are available in the text. GDP - gross domestic product. pcper capita. 2014 cUS\$ - constant 2014 US\$. DAH - Development Assistance for Health. ART - antiretroviral therapy. PEPFAR - President's Emergency Plan for AIDS Relief. ${ }^{\circ}$ dependent variable for regression. ${ }^{*} p<0.05,{ }^{* *} p<0.01,{ }^{* * *} p<0.001$.

\begin{tabular}{|c|c|c|c|c|c|c|c|c|c|}
\hline & $\mathrm{HIV} / \mathrm{AI}$ & HIV\&TB & & & TB & & & Malaria & \\
\hline & & & & & & & & & \\
\hline ln (Funding request pc pa), $2014 \mathrm{cuS}^{\circ}$ & 655 & -0.938 & 1.717 & 466 & -1.733 & 1.433 & 433 & -1.252 & 1.616 \\
\hline Proposal approved $(0 / 1)^{\circ}$ & 655 & 0.446 & 0.497 & 466 & 0.511 & 0.500 & 433 & 0.455 & 0.499 \\
\hline $\operatorname{TRP}^{\prime}$ rating $(1-4)^{\circ}$ & 655 & 2.547 & 0.669 & 466 & 2.455 & 0.700 & 433 & 2.550 & 0.622 \\
\hline $\ln (\mathrm{GDP} P C), 2014$ cUS\$ & 632 & 6.750 & 1.011 & 447 & 6.678 & 0.970 & 411 & 6.387 & 0.909 \\
\hline In (population) & 655 & 16.294 & 1.772 & 466 & 16.389 & 1.584 & 433 & 16.436 & 1.685 \\
\hline UHC tracer $(0-100)$ & 651 & 0.411 & 0.171 & 464 & 0.406 & 0.174 & 433 & 0.345 & 0.139 \\
\hline Dom. health exp, 8 GDP & 612 & 0.050 & 0.025 & 428 & 0.052 & 0.026 & 403 & 0.044 & 0.021 \\
\hline 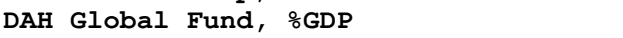 & 491 & 0.002 & 0.005 & 368 & 0.002 & 0.005 & 315 & 0.003 & 0.005 \\
\hline non-Global Fund DAH, & 491 & 0.010 & 0.012 & 367 & 0.010 & 0.012 & 314 & 0.012 & 0.013 \\
\hline Prevalence, \% & 651 & 1.747 & 3.364 & 458 & 0.269 & 0.181 & 433 & 14.000 & 15.653 \\
\hline TB treatment success, $\frac{\circ}{0}$ & 0 & & & 431 & 78.019 & 10.964 & 0 & & \\
\hline Resubmitted proposal $(0 / 1)$ & 655 & 0.392 & 0.489 & 466 & 0.373 & 0.484 & 433 & 0.450 & 0.498 \\
\hline Year of Round $(2002-2010)$ & 655 & 2005.4 & 2.7 & 466 & 2005.6 & 2.6 & 433 & 2005.1 & 2.4 \\
\hline Any grant already approved $(0 / 1)$ & 655 & 0.302 & 0.460 & 466 & 0.399 & 0.490 & 433 & 0.365 & 0.482 \\
\hline Government effectiveness $(-2.5 / 2.5)$ & 632 & -0.561 & 0.567 & 449 & -0.645 & 0.518 & 415 & -0.707 & 0.506 \\
\hline Control of corruption $(-2.5 / 2.5)$ & 636 & -0.603 & 0.528 & 450 & -0.676 & 0.483 & 415 & -0.716 & 0.459 \\
\hline Voice and accountability $(-2.5 / 2.5)$ & 636 & -0.544 & 0.719 & 450 & -0.642 & 0.697 & 415 & -0.682 & 0.668 \\
\hline PEPFAR focus country $(0 / 1)$ & 655 & 0.127 & 0.333 & 0 & & & 0 & & \\
\hline Fragile state $(0 / 1)$ & 655 & 0.380 & 0.486 & 466 & 0.408 & 0.492 & 433 & 0.492 & 0.501 \\
\hline English speaking $(0 / 1)$ & 655 & 0.345 & 0.476 & 466 & 0.320 & 0.467 & 433 & 0.372 & 0.484 \\
\hline Observations & 655 & & & 466 & & & 433 & & \\
\hline
\end{tabular}


Table 35 | Summary statistics for variables in Regression 3, by disease

Data are number of observations (count), mean, and standard deviation (SD). Sample is restricted to grants signed under the rounds-based mechanism (2002-2010). Sources and definitions of the variables are available in the text. av - average. GDP - gross domestic product. pc - per capita. 2014 cUS\$ - constant 2014 US\$. $D A H$ - Development Assistance for Health. ART - antiretroviral therapy. PEPFAR - President's Emergency Plan for AIDS Relief. ${ }^{\circ}$ dependent variable for regression. ${ }^{*} p<0.05,{ }^{* *} p<0.01,{ }^{* * *} p<0.001$

\begin{tabular}{|c|c|c|c|c|c|c|c|c|c|}
\hline & HIV/AIDS, & HIV\&TB & & & TB & & & Malaria & \\
\hline & Count & Mean & SD & Count & Mean & SD & Count & Mean & SD \\
\hline ln(tot signed funding pc RBM), 2014 cuS\$ & 120 & 1.645 & 1.537 & 107 & 0.717 & 1.158 & 80 & 1.397 & 1.454 \\
\hline $\ln (a v$ GDP pc), 2014 cUS\$ & 116 & 7.012 & 1.087 & 102 & 6.787 & 0.952 & 75 & 6.498 & 0.928 \\
\hline $\ln ($ av population) & 120 & 16.045 & 1.642 & 107 & 16.170 & 1.583 & 80 & 16.225 & 1.712 \\
\hline av UHC tracer $(0-100)$ & 119 & 0.505 & 0.173 & 106 & 0.481 & 0.160 & 80 & 0.417 & 0.132 \\
\hline av Dom. health exp, $\frac{\circ G D P}{}$ & 114 & 0.061 & 0.022 & 100 & 0.060 & 0.021 & 74 & 0.054 & 0.019 \\
\hline Global Fund DAH residual, & 116 & 0.001 & 0.002 & 102 & 0.003 & 0.004 & 75 & 0.003 & 0.004 \\
\hline av non-Global Fund DAH, $\circ G D P$ & 116 & 0.009 & 0.012 & 102 & 0.010 & 0.012 & 75 & 0.013 & 0.013 \\
\hline av Prevalence, : & 119 & 0.819 & 1.742 & 105 & 0.249 & 0.177 & 80 & 7.815 & 9.507 \\
\hline av TB treatment success, $\frac{\circ}{0}$ & 0 & & & 102 & 78.269 & 9.319 & 0 & & \\
\hline av Control of corruption $(-2.5 / 2.5)$ & 117 & -0.545 & 0.557 & 104 & -0.662 & 0.467 & 78 & -0.732 & 0.459 \\
\hline av Government effectiveness $(-2.5 / 2.5)$ & 116 & -0.506 & 0.598 & 103 & -0.649 & 0.515 & 77 & -0.745 & 0.507 \\
\hline av Voice and accountability $(-2.5 / 2.5)$ & 116 & -0.498 & 0.746 & 103 & -0.645 & 0.690 & 77 & -0.713 & 0.683 \\
\hline Fragile state $(0-1)$ & 120 & 0.326 & 0.442 & 107 & 0.384 & 0.458 & 80 & 0.488 & 0.464 \\
\hline English speaking $(0 / 1)$ & 120 & 0.267 & 0.444 & 107 & 0.280 & 0.451 & 80 & 0.325 & 0.471 \\
\hline Observations & 120 & & & 107 & & & 80 & & \\
\hline
\end{tabular}


Table 36 | Summary statistics for variables in Regression 4, by disease

Data are number of observations (count), mean, and standard deviation (sd). Sample is restricted to grant proposals from individual countries that were considered by the TRP during the rounds-based mechanism (2002-2010). Sources and definitions of the variables are available in the text. GDP - gross domestic product. pc - per capita. 2014 cUS\$ - constant 2014 US\$. DAH - Development Assistance for Health. ART - antiretroviral therapy. PEPFAR - President's Emergency Plan for AIDS Relief. ${ }^{\circ}$ dependent variable for regression. ${ }^{*} p<0.05,{ }^{* *} p<0.01,{ }^{* * *} p<0.001$.

\begin{tabular}{|c|c|c|c|c|c|c|c|c|c|}
\hline & $\begin{array}{l}\mathrm{HIV} / \mathrm{AI} \\
\text { Coun }\end{array}$ & HIV\&TB & & & TE & & & Malaria & \\
\hline & & & & & & & & & \\
\hline Av Phase 1 perf rating, discrete $(1-5)^{\circ}$ & 413 & 2.990 & 0.911 & 288 & 2.868 & 0.833 & 271 & 3.262 & 0.879 \\
\hline Binary Phase 1 performance rating $(0 / 1)^{\circ}$ & 413 & 0.736 & 0.441 & 288 & 0.705 & 0.457 & 271 & 0.830 & 0.376 \\
\hline In (board approved total pc), 2014 cUS\$ & 291 & 0.552 & 1.646 & 238 & -0.319 & 1.510 & 197 & 0.358 & 1.601 \\
\hline Any grant already approved $(0 / 1)$ & 431 & 0.677 & 0.468 & 297 & 0.778 & 0.416 & 277 & 0.809 & 0.394 \\
\hline Resubmitted proposal $(0 / 1)$ & 431 & 0.473 & 0.500 & 297 & 0.428 & 0.496 & 277 & 0.531 & 0.500 \\
\hline Year of Round $(2002-2010)$ & 431 & 2005.5 & 2.7 & 297 & 2006.0 & 2.5 & 277 & 2006.0 & 2.6 \\
\hline $\ln (\mathrm{GDP} p \mathrm{pc}), 2014$ cUS\$ & 421 & 6.679 & 0.985 & 284 & 6.647 & 0.895 & 263 & 6.261 & 0.831 \\
\hline In (population) & 431 & 16.530 & 1.677 & 297 & 16.536 & 1.689 & 277 & 16.493 & 1.583 \\
\hline UHC tracer $(0-100)$ & 429 & 0.409 & 0.171 & 294 & 0.412 & 0.172 & 277 & 0.340 & 0.133 \\
\hline Dom. health exp, $\because G D P$ & 410 & 0.051 & 0.026 & 274 & 0.054 & 0.027 & 257 & 0.049 & 0.023 \\
\hline DAH Global Fund, $\frac{\circ}{6}$ DDP & 318 & 0.002 & 0.004 & 244 & 0.002 & 0.004 & 219 & 0.003 & 0.005 \\
\hline non-Global Fund DAH, 8 GDP & 318 & 0.010 & 0.012 & 243 & 0.009 & 0.012 & 217 & 0.014 & 0.013 \\
\hline Prevalence, $\frac{\circ}{0}$ & 429 & 1.923 & 3.355 & 290 & 0.280 & 0.179 & 277 & 15.541 & 15.600 \\
\hline Prevalence, $\frac{\circ}{0}$ & 429 & 1.923 & 3.355 & 290 & 0.280 & 0.179 & 277 & 15.541 & 15.600 \\
\hline Prevalence, $\frac{\circ}{6}$ & 429 & 1.923 & 3.355 & 290 & 0.280 & 0.179 & 277 & 15.541 & 15.600 \\
\hline Phase 1 change mortality, abs & 417 & -0.011 & 0.033 & 292 & -0.001 & 0.002 & 270 & -0.009 & 0.015 \\
\hline Phase 1 change mortality, abs & 417 & -0.011 & 0.033 & 292 & -0.001 & 0.002 & 270 & -0.009 & 0.015 \\
\hline Phase 1 change mortality, abs & 417 & -0.011 & 0.033 & 292 & -0.001 & 0.002 & 270 & -0.009 & 0.015 \\
\hline TB treatment success, $\frac{\circ}{0}$ & 0 & & & 273 & 78.923 & 10.791 & 0 & & \\
\hline P1 change TB treatm success, abs & 0 & & & 267 & 1.951 & 6.698 & 0 & & \\
\hline Government effectiveness $(-2.5 / 2.5)$ & 419 & -0.548 & 0.532 & 284 & -0.622 & 0.507 & 264 & -0.734 & 0.481 \\
\hline Control of corruption $(-2.5 / 2.5)$ & 420 & -0.610 & 0.498 & 285 & -0.693 & 0.450 & 264 & -0.743 & 0.456 \\
\hline Voice and accountability $(-2.5 / 2.5)$ & 420 & -0.494 & 0.694 & 285 & -0.621 & 0.679 & 264 & -0.668 & 0.657 \\
\hline Fragile state $(0 / 1)$ & 431 & 0.346 & 0.476 & 297 & 0.391 & 0.489 & 277 & 0.502 & 0.501 \\
\hline English speaking $(0 / 1)$ & 431 & 0.357 & 0.480 & 297 & 0.327 & 0.470 & 277 & 0.390 & 0.489 \\
\hline TRP rating $=1$ & 431 & 0.146 & 0.354 & 297 & 0.148 & 0.356 & 277 & 0.090 & 0.287 \\
\hline $10 \mathrm{~ns}$ & 431 & & & 297 & & & 277 & & \\
\hline
\end{tabular}


Table 37 | Robustness of predictors of In(funding request per capita), by disease

Data are OLS regression coefficients ( $p$-values) (Regression 1). Columns 1, 4, 7 describe full models for each disease; columns 2, 5, 8 describe intermediate models with $p<0.2$ but including all TRP-related variables regardless of their significance; 3, 6, 9 columns comprise reduced models retaining variables with $p<0.2$. Sample is restricted to grant proposals from individual countries that were considered by the TRP during the rounds-based mechanism (2002-2010) and for which requested funding volumes are recorded. Sources and definitions of the variables are available in the text. GDP - gross domestic product. pc - per capita. 2014 cUS $\$$ - constant 2014 US\$. DAH - Development Assistance for Health. PEPFAR - President's Emergency Plan for AIDS Relief. ${ }^{*} p<0.05,{ }^{* *} p<0.01,{ }^{* * *} p<0.001$.

Dependent variable: In(Funding request per capita), 2014 constant US\$

\begin{tabular}{|c|c|c|c|c|c|c|c|c|c|}
\hline & $\begin{array}{r}(1) \\
\text { HIV1 }\end{array}$ & $\begin{array}{r}(2) \\
\text { HIV } 2 \\
\end{array}$ & $\begin{array}{r}(3) \\
\text { HIV3 }\end{array}$ & $\begin{array}{l}(4) \\
\text { TB1 }\end{array}$ & $\begin{array}{l}(5) \\
\text { TB2 }\end{array}$ & $\begin{array}{l}(6) \\
\text { TB3 }\end{array}$ & $\begin{array}{r}(7) \\
\text { Malaria1 }\end{array}$ & $\begin{array}{r}(8) \\
\text { Malaria2 }\end{array}$ & $\begin{array}{r}(9) \\
\text { Malaria3 }\end{array}$ \\
\hline $\ln (G D P$ pc), 2014 cUS\$ & $\begin{array}{l}-0.0331 \\
(0.691)\end{array}$ & & & $\begin{array}{r}-0.00212 \\
(0.981)\end{array}$ & & & $\begin{array}{l}0.0918 \\
(0.293)\end{array}$ & & \\
\hline ln (population) & $\begin{array}{l}-0.6786 * \star * \\
(0.000)\end{array}$ & $\begin{array}{l}-0.6929 * * * \\
(0.000)\end{array}$ & $\begin{array}{l}-0.6942 * \star * \\
(0.000)\end{array}$ & $\begin{array}{l}-0.5616 * \star * \\
(0.000)\end{array}$ & $\begin{array}{l}-0.5739 * \star \star \\
(0.000)\end{array}$ & $\begin{array}{l}-0.5744 * \star * \\
(0.000)\end{array}$ & $\begin{array}{l}-0.6636 * * * \\
(0.000)\end{array}$ & $\begin{array}{l}-0.6947 * \star \star \\
(0.000)\end{array}$ & $\begin{array}{l}-0.6951 * \star * \\
(0.000)\end{array}$ \\
\hline UHC tracer $(0-100)$ & $\begin{array}{l}-1.028^{\star} \\
(0.010)\end{array}$ & $\begin{array}{l}-0.920 * \star \\
(0.003)\end{array}$ & $\begin{array}{l}-0.912 \star \star \\
(0.003)\end{array}$ & $\begin{array}{l}0.989 * \\
(0.031)\end{array}$ & $\begin{array}{l}0.859 * \star \\
(0.008)\end{array}$ & $\begin{array}{l}0.819 * \\
(0.011)\end{array}$ & $\begin{array}{l}-2.376 \star \star \star \\
(0.000)\end{array}$ & $\begin{array}{l}-2.239 * \star \star \\
(0.000)\end{array}$ & $\begin{array}{l}-2.248^{* \star *} \\
(0.000)\end{array}$ \\
\hline Dom. health exp, $\% G D P$ & $\begin{array}{r}1.932 \\
(0.344)\end{array}$ & & & $\begin{array}{l}-0.0404 \\
(0.985)\end{array}$ & & & $\begin{array}{l}-1.564 \\
(0.526)\end{array}$ & & \\
\hline DAH Global Fund, $\% G D P$ & $\begin{array}{l}40.80 * * \\
(0.001)\end{array}$ & $\begin{array}{l}43.99 * * * \\
(0.000)\end{array}$ & $\begin{array}{l}43.70 * \star \star \\
(0.000)\end{array}$ & $\begin{array}{r}2.990 \\
(0.822)\end{array}$ & & & $\begin{array}{r}9.502 \\
(0.485)\end{array}$ & & \\
\hline non-Global Fund DAH， $\%$ GDP & $\begin{array}{r}1.406 \\
(0.781)\end{array}$ & & & $\begin{array}{r}4.593 \\
(0.380)\end{array}$ & & & $\begin{array}{l}18.33 * \star * \\
(0.001)\end{array}$ & $\begin{array}{l}16.97 * * \star \\
(0.000)\end{array}$ & $\begin{array}{l}16.63 * * * \\
(0.000)\end{array}$ \\
\hline $\begin{array}{l}\text { Prevalence, } \frac{\circ}{1} \\
\text { TB treatment success, \% }\end{array}$ & $\begin{array}{l}0.0980 * * * \\
(0.000)\end{array}$ & $\begin{array}{l}0.100 * \star * \\
(0.000)\end{array}$ & $\begin{array}{l}0.100 * * * \\
(0.000)\end{array}$ & $\begin{array}{l}1.214 * \star \star \\
(0.000) \\
-0.00694\end{array}$ & $\begin{array}{l}1.220 * * * \\
(0.000) \\
-0.00628\end{array}$ & $\begin{array}{l}1.179 * * * \\
(0.000) \\
-0.00615\end{array}$ & $\begin{array}{l}0.0222 * * * \\
(0.000)\end{array}$ & $\begin{array}{l}0.0231 * * \star \\
(0.000)\end{array}$ & $\begin{array}{l}0.0232 * * * \\
(0.000)\end{array}$ \\
\hline 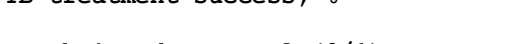 & & & & $(0.128)$ & $(0.149)$ & $(0.158)$ & & & \\
\hline Resubmitted proposal (0/1) & $\begin{array}{l}-0.0341 \\
(0.672)\end{array}$ & $\begin{array}{l}-0.0471 \\
(0.556)\end{array}$ & & $\begin{array}{l}0.118 \\
(0.180)\end{array}$ & $\begin{array}{l}0.108 \\
(0.210)\end{array}$ & & $\begin{array}{l}0.0643 \\
(0.483)\end{array}$ & $\begin{array}{r}0.0713 \\
(0.430)\end{array}$ & \\
\hline Year of Round (2002-2010) & $\begin{array}{l}0.145 * \star \star \\
(0.000)\end{array}$ & $\begin{array}{l}0.152 * \star \star \\
(0.000)\end{array}$ & $\begin{array}{l}0.151 * \star \star \\
(0.000)\end{array}$ & $\begin{array}{l}0.225 * \star \star \\
(0.000)\end{array}$ & $\begin{array}{l}0.232 * \star \star \\
(0.000)\end{array}$ & $\begin{array}{l}0.228 * \star * \\
(0.000)\end{array}$ & $\begin{array}{l}0.267 * \star \star \\
(0.000)\end{array}$ & $\begin{array}{l}0.269 * \star \star \\
(0.000)\end{array}$ & $\begin{array}{l}0.268 * \star \star \\
(0.000)\end{array}$ \\
\hline Any grant already approved $(0 / 1)$ & $\begin{array}{l}-0.0628 \\
(0.466)\end{array}$ & $\begin{array}{l}-0.0396 \\
(0.639)\end{array}$ & & $\begin{array}{l}-0.149 \\
(0.100)\end{array}$ & $\begin{array}{l}-0.146 \\
(0.098)\end{array}$ & $\begin{array}{l}-0.124 \\
(0.150)\end{array}$ & $\begin{array}{r}0.131 \\
(0.177)\end{array}$ & $\begin{array}{r}0.122 \\
(0.206)\end{array}$ & $\begin{array}{r}0.131 \\
(0.170)\end{array}$ \\
\hline Government effectiveness $(-2.5 / 2.5)$ & $\begin{array}{l}-0.427 * * \\
(0.007)\end{array}$ & $\begin{array}{l}-0.291 * * \\
(0.006)\end{array}$ & $\begin{array}{l}-0.290 * * \\
(0.006)\end{array}$ & $\begin{array}{l}-0.577^{* * *} \\
(0.001)\end{array}$ & $\begin{array}{l}-0.596 * \star \star \\
(0.000)\end{array}$ & $\begin{array}{l}-0.613^{\star \star \star *} \\
(0.000)\end{array}$ & $\begin{array}{l}-0.216 \\
(0.289)\end{array}$ & & \\
\hline Control of corruption $(-2.5 / 2.5)$ & $\begin{array}{r}0.170 \\
(0.240)\end{array}$ & & & $\begin{array}{r}0.243 \\
(0.106)\end{array}$ & $\begin{array}{c}0.256 \\
(0.085)\end{array}$ & $\begin{array}{r}0.260 \\
(0.080)\end{array}$ & $\begin{array}{r}0.199 \\
(0.266)\end{array}$ & & \\
\hline $\begin{array}{l}\text { Voice and accountability }(-2.5 / 2.5) \\
\text { PEPFAR focus country }(0 / 1)\end{array}$ & $\begin{array}{l}0.144 \\
(0.068) \\
0.551 * \star * \\
(0.000)\end{array}$ & $\begin{array}{l}0.184 * \\
(0.012) \\
0.555 * \star \star \\
(0.000)\end{array}$ & $\begin{array}{l}0.184 * \\
(0.012) \\
0.557 \star \star \star \\
(0.000)\end{array}$ & $\begin{array}{r}0.156 \\
(0.055)\end{array}$ & $\begin{array}{l}0.171 * \\
(0.026)\end{array}$ & $\begin{array}{c}0.174 * \\
(0.023)\end{array}$ & $\begin{array}{l}0.231 * \\
(0.011)\end{array}$ & $\begin{array}{l}0.192 * * \\
(0.010)\end{array}$ & $\begin{array}{l}0.192 \star * \\
(0.010)\end{array}$ \\
\hline Fragile state $(0 / 1)$ & $\begin{array}{l}-0.146 \\
(0.203)\end{array}$ & & & $\begin{array}{l}-0.0185 \\
(0.876)\end{array}$ & & & $\begin{array}{c}0.0752 \\
(0.562)\end{array}$ & & \\
\hline $\begin{array}{l}\text { English speaking }(0 / 1) \\
\text { Constant }\end{array}$ & $\begin{array}{l}-0.00498 \\
(0.963) \\
-280.0 \star \star \star \\
(0.000)\end{array}$ & $\begin{array}{l}-295.4^{\star \star \star *} \\
(0.000)\end{array}$ & $\begin{array}{l}-292.9 * \star \star \\
(0.000)\end{array}$ & $\begin{array}{l}0.117 \\
(0.259) \\
-444.0 * \star \star\end{array}$ & $\begin{array}{l}0.144 \\
(0.139) \\
-458.2 * \star \star\end{array}$ & $\begin{array}{l}0.147 \\
(0.132) \\
-450.2 \star \star \star\end{array}$ & $\begin{array}{l}0.272 \star \\
(0.014) \\
-526.9 * \star \star\end{array}$ & $\begin{array}{l}0.284 * \star \\
(0.006) \\
-529.7 \star \star \star \\
(0.000)\end{array}$ & $\begin{array}{l}0.288 * \star \\
(0.005) \\
-526.4 \star \star \star\end{array}$ \\
\hline & & & & & & & & & \\
\hline & & & & & & & & & \\
\hline $\begin{array}{l}\text { Adjusted R-squared } \\
\text { Observations }\end{array}$ & $\begin{array}{r}0.751 \\
465\end{array}$ & $\begin{array}{r}0.752 \\
465\end{array}$ & $\begin{array}{r}0.752 \\
465\end{array}$ & $\begin{array}{r}0.710 \\
334\end{array}$ & $\begin{array}{r}0.714 \\
334\end{array}$ & $\begin{array}{r}0.713 \\
334\end{array}$ & $\begin{array}{r}0.784 \\
299\end{array}$ & $\begin{array}{r}0.786 \\
299\end{array}$ & $\begin{array}{r}0.787 \\
299\end{array}$ \\
\hline & & & 465 & 334 & 334 & 334 & 299 & 299 & 299 \\
\hline
\end{tabular}




\section{Table 38 | Robustness of predictors for TRP rating, by disease}

Data are logistic regression odds ratios (t-statistic) without constant (Regression 2). Columns 1, 4, 7 describe full models for each disease; columns 2, 5, 8 describe intermediate models with $p<0.2$ but including all TRP-related variables regardless of their significance; 3, 6, 9 columns comprise reduced models retaining variables with $p<0$.2. Sample is restricted to grant proposals from individual countries that were considered by the TRP during the rounds-based mechanism (2002-2010).

Sources and definitions of the variables are available in the appendix. GDP - gross domestic product. pc-per capita. 2014 cUS\$ - constant 2014 US\$. DAH - Development Assistance for Health. UHC - Universal Health Coverage. PEPFAR - President's Emergency Plan for AIDS Relief. ${ }^{*} p<0.05,{ }^{* *} p<0.01,{ }^{* * *} p<0.001$.

\begin{tabular}{|c|c|c|c|c|c|c|c|c|c|}
\hline & $\begin{array}{r}(1) \\
\text { HIV1 }\end{array}$ & $\begin{array}{r}(2) \\
\text { HIV } 2\end{array}$ & $\begin{array}{r}(3) \\
\text { HIV3 }\end{array}$ & $\begin{array}{l}(4) \\
\text { TB1 }\end{array}$ & $\begin{array}{l}(5) \\
\text { TB2 }\end{array}$ & $\begin{array}{l}\text { (6) } \\
\text { TB3 }\end{array}$ & $\begin{array}{r}\text { (7) } \\
\text { Malaria1 }\end{array}$ & $\begin{array}{r}(8) \\
\text { Malaria2 }\end{array}$ & $\begin{array}{r}(9) \\
\text { Malaria3 }\end{array}$ \\
\hline $\ln (G D P$ pc), 2014 cUS\$ & $\begin{array}{l}-0.6928 * \\
(0.015)\end{array}$ & $\begin{array}{l}-0.6935 * \star \star \\
(0.000)\end{array}$ & $\begin{array}{l}-0.6935 * \star \star \\
(0.000)\end{array}$ & $\begin{array}{r}0.0072 \\
(0.982)\end{array}$ & & & $\begin{array}{r}0.6337 \\
(0.098)\end{array}$ & $0.6336^{*}$ & $\begin{array}{l}0.6336 * \\
(0.042)\end{array}$ \\
\hline In (population) & 0.0686 & & & -0.0459 & & & 0.0985 & & \\
\hline & $(0.621)$ & & & $(0.777)$ & & & $(0.670)$ & & \\
\hline UHC tracer $(0-100)$ & 0.4238 & & & -2.0518 & & & 1.9092 & & \\
\hline & $(0.757)$ & & & $(0.227)$ & & & $(0.418)$ & & \\
\hline Dom. health exp, \&GDP & 6.4955 & & & 3.5215 & & & -2.6064 & & \\
\hline & $(0.364)$ & & & $(0.680)$ & & & $(0.814)$ & & \\
\hline DAH Global Fund, \%GDP & $\begin{array}{l}-8.358 \\
(0.854)\end{array}$ & & & $96.963^{*}$ & $78.694 *$ & $81.315^{\star}$ & $\begin{array}{l}48.620 \\
(0.412)\end{array}$ & & \\
\hline non-Global Fund DAH, $: G D P$ & -14.356 & -19.643 & -19.643 & -15.880 & & & 23.552 & 28.612 & 28.612 \\
\hline & $(0.402)$ & $(0.192)$ & $(0.192)$ & $(0.429)$ & & & $(0.329)$ & $(0.193)$ & $(0.193)$ \\
\hline Prevalence, $\frac{\circ}{0}$ & 0.0488 & & & -1.4074 & & & 0.0295 & 0.0284 & 0.0284 \\
\hline TB treatment success, $\frac{\circ}{\circ}$ & $(0.413)$ & & & $\begin{array}{r}(0.188) \\
0.0177\end{array}$ & & & $(0.071)$ & $(0.051)$ & $(0.051)$ \\
\hline In (board approved total pc), 2014 cuS\$ & -0.2196 & $-0.2089 *$ & $-0.2089 *$ & $\begin{array}{l}(0.280) \\
-0.2411\end{array}$ & $-0.3325 *$ & $-0.3393 *$ & -0.4490 & $-0.4898 * * *$ & 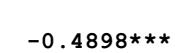 \\
\hline & $(0.150)$ & $(0.017)$ & $(0.017)$ & $(0.236)$ & $(0.012)$ & $(0.010)$ & $(0.079)$ & 10.0 & $(0.001)$ \\
\hline Resubmitted proposal (0/1) & $-0.6100^{*}$ & $-0.6507 *$ & $-0.6507 \star$ & -0.4788 & -0.3571 & & $-1.3640 * \star$ & $-1.3851 \star \star$ & $-1.3851 \star \star$ \\
\hline Year of Round (2002-2010) & $0.2534 * *$ & $0.2868 * \star \star$ & $0.2868 * \star \star$ & $0.2417 \star$ & $0.2707 \star \star$ & $0.2847 * \star *$ & $\begin{array}{r}0.004) \\
0.1874\end{array}$ & $0.2594 *$ & $\begin{array}{l}(0.003) \\
0.2594 *\end{array}$ \\
\hline & $(0.003)$ & $(0.000)$ & $(0.000)$ & $(0.036)$ & $(0.002)$ & $(0.001)$ & $(0.252)$ & $(0.026)$ & $(0.026)$ \\
\hline Any grant already approved (0/1) & $-7.3382 \star \star \star$ & $-7.1996 \star \star \star$ & $-7.1996 * \star \star *$ & $-8.0139 * \star \star *$ & $-7.9512 \star \star \star$ & $-7.9893 \star \star \star *$ & $-8.7428 * \star \star$ & $-8.6365 \star \star \star$ & $-8.6365 * \star *$ \\
\hline Government effectiveness $(-2.5 / 2.5)$ & $\begin{array}{l}(0.000) \\
-0.3991\end{array}$ & $(0.000)$ & $(0.000)$ & $\begin{array}{r}(0.000) \\
0.1289\end{array}$ & $(0.000)$ & $(0.000)$ & $\begin{array}{l}(0.000) \\
-0.4062\end{array}$ & $(0.000)$ & $(0.000)$ \\
\hline & $(0.458)$ & & & $(0.852)$ & & & $(0.634)$ & & \\
\hline Control of corruption $(-2.5 / 2.5)$ & 0.0521 & & & 0.9155 & $0.6939 *$ & $0.7301 *$ & 0.1045 & & \\
\hline & $(0.915)$ & & & $(0.107)$ & $(0.043)$ & $(0.033)$ & $(0.889)$ & & \\
\hline Voice and accountability $(-2.5 / 2.5)$ & 0.3467 & 0.3521 & 0.3521 & -0.1210 & & & $0.7924 *$ & 0.6577 & $\begin{array}{r}0.6577 \\
(0.064)\end{array}$ \\
\hline PEPFAR focus country $(0 / 1)$ & $\begin{array}{r}(0.195) \\
0.6470 \\
(0.180)\end{array}$ & $\begin{array}{l}(0.116) \\
0.9847 \\
(0.012)\end{array}$ & $\begin{array}{l}(0.116) \\
0.9847 * \\
(0.012)\end{array}$ & & & & & & \\
\hline Fragile state $(0 / 1)$ & $\begin{array}{l}-0.1709 \\
(0.665)\end{array}$ & & & $\begin{array}{r}0.1474 \\
(0.734)\end{array}$ & & & $\begin{array}{r}0.8939 \\
(0.118)\end{array}$ & $\begin{array}{r}0.8419 \\
(0.096)\end{array}$ & $\begin{array}{r}0.8419 \\
(0.096)\end{array}$ \\
\hline English speaking $(0 / 1)$ & $\begin{array}{c}0.3068 \\
(0.406)\end{array}$ & & & $\begin{array}{r}0.0108 \\
(0.978)\end{array}$ & & & $\begin{array}{l}-1.2704^{*} \\
(0.011)\end{array}$ & $\begin{array}{l}-1.1668 * \star \\
(0.009)\end{array}$ & $\begin{array}{l}-1.1668 * \star \\
(0.009)\end{array}$ \\
\hline & 0.517 & 0.513 & 0.513 & 0.537 & 0.530 & 0.528 & 0.636 & 0.633 & 0.633 \\
\hline Obse: & 464 & 464 & 464 & 334 & 334 & 334 & 299 & 299 & 299 \\
\hline
\end{tabular}


Table 39 | Robustness of predictors for In(total Global Fund funding pc), by disease

Data are OLS regression coefficients ( $p$-values) (Regression 3). Columns 1, 3, 5 describe full models for each disease and columns 2, 4, 6 comprise reduced models retaining variables with $p<0.2$. Sample is restricted to grants approved under the rounds-based mechanism (2002-2010). Sources and definitions of the variables are available in the appendix. GDP - gross domestic product. pc - per capita. 2014 cUS\$ - constant 2014 US\$. DAH - Development Assistance for Health. PEPFAR President's Emergency Plan for AIDS Relief. ${ }^{*} p<0.05,{ }^{* *} p<0.01,{ }^{* * *} p<0.001$.

Dependent variable: In(total signed funding per capita) rounds-based mechanism, 2014 constant US\$

\begin{tabular}{|c|c|c|c|c|c|c|}
\hline & $\begin{array}{r}(1) \\
\text { HIV1 }\end{array}$ & $\begin{array}{r}(2) \\
\text { HIV3 }\end{array}$ & $\begin{array}{l}(3) \\
\text { TB1 }\end{array}$ & $\begin{array}{l}(4) \\
\text { TB3 }\end{array}$ & $\begin{array}{r}(5) \\
\text { Malaria1 }\end{array}$ & $\begin{array}{r}(6) \\
\text { Malaria3 }\end{array}$ \\
\hline $\ln ($ av GDP pc), 2014 cUS\$ & $\begin{array}{l}-0.4521 * \\
(0.022)\end{array}$ & $\begin{array}{l}-0.4007 \star \star \\
(0.008)\end{array}$ & $\begin{array}{l}-0.3205 \\
(0.081)\end{array}$ & $\begin{array}{l}-0.2980 * \\
(0.020)\end{array}$ & $\begin{array}{l}-0.0518 \\
(0.744)\end{array}$ & \\
\hline $\ln ($ av population) & 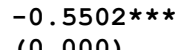 & 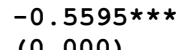 & $-0.4719 * \star \star$ & $-0.4763 * \star \star$ & $-0.5633 * \star \star$ & $-0.5525 * \star \star$ \\
\hline av UHC tracer $(0-100)$ & $\begin{array}{r}(0.000) \\
0.5223 \\
(0.602)\end{array}$ & $(0.000)$ & $\begin{array}{l}(0.000) \\
2.3904 * \\
(0.020)\end{array}$ & $\begin{array}{l}(0.000) \\
2.7051 * \star \\
(0.001)\end{array}$ & $\begin{array}{l}(0.000) \\
-3.2203 * * * \\
(0.001)\end{array}$ & $\begin{array}{l}(0.000) \\
-3.1680 * \star \star \\
(0.000)\end{array}$ \\
\hline av Dom. health exp, ${ }^{\circ} G D P$ & $\begin{array}{r}4.2055 \\
(0.422)\end{array}$ & & $\begin{array}{l}-0.7168 \\
(0.869)\end{array}$ & (0.0 & $\begin{array}{l}-2.5929 \\
(0.582)\end{array}$ & \\
\hline Global Fund DAH residual, $\%$ GDP & $\begin{array}{r}82.632 \\
(0.257)\end{array}$ & $\begin{array}{r}76.758 \\
(0.185)\end{array}$ & $\begin{array}{r}20.052 \\
(0.541)\end{array}$ & & $\begin{array}{l}37.910 \\
(0.378)\end{array}$ & \\
\hline av non-Global Fund DAH, $\frac{\circ}{\circ}$ GDP & $\begin{array}{l}-2.064 \\
(0.900)\end{array}$ & & $\begin{array}{l}-8.261 \\
(0.484)\end{array}$ & & $\begin{array}{l}19.398 \\
(0.117)\end{array}$ & $\begin{array}{l}26.391 * * * \\
(0.000)\end{array}$ \\
\hline av Prevalence, $\frac{\circ}{0}$ & $\begin{array}{l}0.1768 * \\
(0.027)\end{array}$ & $\begin{array}{l}0.1672 \star \\
(0.014)\end{array}$ & $\begin{array}{l}1.8773 * \star \star \\
(0.001)\end{array}$ & $\begin{array}{l}1.9823 * * * \\
(0.000)\end{array}$ & $\begin{array}{l}0.0440 * \star * \\
(0.000)\end{array}$ & $\begin{array}{l}0.0405 * \star * \\
(0.000)\end{array}$ \\
\hline av TB treatment success, $\frac{\circ}{0}$ & & & $\begin{array}{l}0.0031 \\
(0.756)\end{array}$ & & & \\
\hline av Control of corruption $(-2.5 / 2.5)$ & $\begin{array}{l}-0.1758 \\
(0.649)\end{array}$ & & $\begin{array}{l}-0.2831 \\
(0.398)\end{array}$ & $\begin{array}{l}-0.3349 \\
(0.094)\end{array}$ & $\begin{array}{l}-0.1246 \\
(0.747)\end{array}$ & \\
\hline av Government effectiveness $(-2.5 / 2.5)$ & $\begin{array}{l}-0.4891 \\
(0.268)\end{array}$ & $\begin{array}{l}-0.4496 \\
(0.052)\end{array}$ & $\begin{array}{l}-0.2498 \\
(0.499)\end{array}$ & & $\begin{array}{r}0.2472 \\
(0.602)\end{array}$ & \\
\hline av Voice and accountability $(-2.5 / 2.5)$ & $\begin{array}{r}0.1614 \\
(0.390)\end{array}$ & & $\begin{array}{r}0.0563 \\
(0.739)\end{array}$ & & $\begin{array}{l}-0.1397 \\
(0.446)\end{array}$ & \\
\hline av PEPFAR focus country $(0-1)$ & $\begin{array}{l}1.3285^{\star \star} \\
(0.009)\end{array}$ & $\begin{array}{l}1.3919 \star \star \\
(0.002)\end{array}$ & & & & \\
\hline Fragile state $(0-1)$ & $\begin{array}{l}-0.7395 * \\
(0.025)\end{array}$ & $\begin{array}{l}-0.7986 * \\
(0.010)\end{array}$ & $\begin{array}{l}-0.3502 \\
(0.206)\end{array}$ & & $\begin{array}{l}-0.2222 \\
(0.416)\end{array}$ & \\
\hline English speaking $(0 / 1)$ & $\begin{array}{r}0.1425 \\
(0.622)\end{array}$ & & $\begin{array}{l}0.5373^{\star} \\
(0.013)\end{array}$ & $\begin{array}{l}0.5019 * \star \\
(0.008)\end{array}$ & $\begin{array}{l}0.4968 * \\
(0.024)\end{array}$ & $\begin{array}{l}0.5795^{\star \star} \\
(0.002)\end{array}$ \\
\hline Constant & $\begin{array}{l}12.6355 * \star \star \\
(0.000)\end{array}$ & $\begin{array}{l}13.0268 * \star \star \\
(0.000)\end{array}$ & $\begin{array}{l}8.3533 * * \star \\
(0.000)\end{array}$ & $\begin{array}{l}8.2403 * \star \star \\
(0.000)\end{array}$ & $\begin{array}{l}11.5731 * \star \star \\
(0.000)\end{array}$ & $\begin{array}{l}10.8118 * \star \star \\
(0.000)\end{array}$ \\
\hline$\pi$ & 0.653 & 0.643 & 0.632 & 0.621 & 0.824 & 0.813 \\
\hline Adjusted R-squared & 0.607 & 0.619 & 0.575 & 0.595 & 0.788 & 0.798 \\
\hline Observations & 111 & 111 & 97 & 97 & 72 & 72 \\
\hline
\end{tabular}


Table 40 | Robustness of predictors for average Phase 1 performance, by disease

Data are ordered logistic regression odds ratios (p-values) without constant (Regression 4). Columns 1, 4, 7 describe full models for each disease; columns 2, 5, 8 describe intermediate models with $p<0.2$ but including all TRP-related variables regardless of their significance; 3, 6, 9 columns comprise reduced models retaining variables with $p<0.2$. Sample is restricted to grant proposals from individual countries that were approved during the rounds-based mechanism (2002-2010). Sources and definitions of the variables are available in the appendix. GDP - gross domestic product. pc - per capita. 2014 cUS\$ - constant 2014 US\$. TRP - Technical Review Panel. DAH - Development Assistance for Health. ART - antiretroviral therapy. UHC - Universal Health Coverage. LFA - Local Fund Agent. PwC - PriceWaterHouse Coopers. STPH - Swiss Tropical and Public Health Institute. UNOPS - United Nations Operations and Project Services. PR - Principal Recipient. CSO Civil Society Organization. PEPFAR - President's Emergency Plan for AIDS Relief. ${ }^{*} p<0.05,{ }^{* *} p<0.01,{ }^{* * *} p<0.001$.

Dependent variable: Phase 1 Performance rating (1-5), odds ratios

\begin{tabular}{|c|c|c|c|c|c|c|c|c|c|}
\hline & $\begin{array}{r}(1) \\
\text { HIV1 }\end{array}$ & $\begin{array}{r}(2) \\
\text { HIV2 }\end{array}$ & $\begin{array}{r}(3) \\
\text { HIV3 }\end{array}$ & $\begin{array}{l}(4) \\
\text { TB1 }\end{array}$ & $\begin{array}{l}(5) \\
\text { TB2 }\end{array}$ & $\begin{array}{l}(6) \\
\text { TB3 }\end{array}$ & $\begin{array}{r}(7) \\
\text { Malaria1 }\end{array}$ & $\begin{array}{r}(8) \\
\text { Malaria2 }\end{array}$ & $\begin{array}{r}(9) \\
\text { Malaria3 }\end{array}$ \\
\hline $\mathrm{TRP}$ rating $=1$ & $\begin{array}{l}-0.4259 \\
(0.549)\end{array}$ & $\begin{array}{l}-0.2703 \\
(0.696)\end{array}$ & & $\begin{array}{l}-0.0727 \\
(0.895)\end{array}$ & $\begin{array}{l}-0.1227 \\
(0.813)\end{array}$ & & $\begin{array}{r}0.4926 \\
(0.547)\end{array}$ & $\begin{array}{r}0.5163 \\
(0.512)\end{array}$ & \\
\hline In (board approved total pc), 2014 cuS\$ & $\begin{array}{r}0.2481 \\
(0.146)\end{array}$ & $\begin{array}{l}0.2345^{\star} \\
(0.018)\end{array}$ & $\begin{array}{l}0.2300 * \\
(0.021)\end{array}$ & $\begin{array}{l}-0.0106 \\
(0.959)\end{array}$ & $\begin{array}{l}-0.0869 \\
(0.485)\end{array}$ & & $\begin{array}{l}-0.1135 \\
(0.611)\end{array}$ & $\begin{array}{l}-0.1239 \\
(0.367)\end{array}$ & \\
\hline Any grant already approved $(0 / 1)$ & $\begin{array}{l}-0.6661 \\
(0.159)\end{array}$ & $\begin{array}{l}-0.7615 \\
(0.091)\end{array}$ & $\begin{array}{l}-0.8073 \\
(0.069)\end{array}$ & $\begin{array}{r}0.2165 \\
(0.712)\end{array}$ & $\begin{array}{l}0.2278 \\
(0.678)\end{array}$ & & $\begin{array}{l}-0.5034 \\
(0.502)\end{array}$ & $\begin{array}{l}-0.4598 \\
(0.515)\end{array}$ & \\
\hline Resubmitted proposal $(0 / 1)$ & $\begin{array}{r}0.1224 \\
(0.684)\end{array}$ & $\begin{array}{r}0.1775 \\
(0.538)\end{array}$ & & $\begin{array}{l}-0.3058 \\
(0.373)\end{array}$ & $\begin{array}{l}-0.3276 \\
(0.303)\end{array}$ & & $\begin{array}{l}-0.0840 \\
(0.820)\end{array}$ & $\begin{array}{l}-0.1270 \\
(0.720)\end{array}$ & \\
\hline Year of Round $(2002-2010)$ & $\begin{array}{r}0.1584 \\
(0.137)\end{array}$ & $\begin{array}{l}0.1698^{*} \\
(0.035)\end{array}$ & $\begin{array}{l}0.1730 * \\
(0.030)\end{array}$ & $\begin{array}{r}0.0983 \\
(0.420)\end{array}$ & $\begin{array}{c}0.1154 \\
(0.272)\end{array}$ & & $\begin{array}{r}0.2421 \\
(0.081)\end{array}$ & $\begin{array}{l}0.2403^{*} \\
(0.027)\end{array}$ & $\begin{array}{l}0.1961 * \\
(0.037)\end{array}$ \\
\hline PEPFAR focus country $(0 / 1)$ & $\begin{array}{l}-0.4922 \\
(0.339)\end{array}$ & & & & & & & & \\
\hline Fragile state $(0 / 1)$ & $\begin{array}{r}0.4535 \\
(0.327)\end{array}$ & & & $\begin{array}{l}-0.3676 \\
(0.453)\end{array}$ & & & $\begin{array}{r}0.1215 \\
(0.819)\end{array}$ & & \\
\hline $\begin{array}{l}\text { English speaking }(0 / 1) \\
\text { Additional control vari }\end{array}$ & $\begin{array}{l}-0.2715 \\
(0.517)\end{array}$ & & & $\begin{array}{l}-0.1397 \\
(0.764)\end{array}$ & & & $\begin{array}{l}-0.9301 \\
(0.052)\end{array}$ & $\begin{array}{l}-0.8633 * \\
(0.040)\end{array}$ & $\begin{array}{l}-0.7724 \\
(0.056)\end{array}$ \\
\hline $\ln ($ GDP $\mathrm{pC}), 2014$ cUS\$ & $\begin{array}{r}0.4244 \\
(0.144)\end{array}$ & $\begin{array}{c}0.3036 \\
(0.136)\end{array}$ & $\begin{array}{c}0.2838 \\
(0.159)\end{array}$ & $\begin{array}{l}-0.0743 \\
(0.852)\end{array}$ & & & $\begin{array}{l}1.1751 * \star \\
(0.001)\end{array}$ & $\begin{array}{l}1.0766 * \star * \\
(0.001)\end{array}$ & $\begin{array}{l}0.9709 * * \\
(0.001)\end{array}$ \\
\hline In (population) & $\begin{array}{r}0.0135 \\
(0.932)\end{array}$ & & & $\begin{array}{r}0.0079 \\
(0.966)\end{array}$ & & & $\begin{array}{r}0.0333 \\
(0.878)\end{array}$ & & \\
\hline UHC tracer $(0-100)$ & $\begin{array}{l}-3.5492 * \\
(0.012)\end{array}$ & $\begin{array}{l}-4.2110 * \star \star \\
(0.000)\end{array}$ & $\begin{array}{l}-4.2189 \star \star \star \\
(0.000)\end{array}$ & $\begin{array}{l}-1.6650 \\
(0.384)\end{array}$ & & $\begin{array}{l}-2.0587 \\
(0.075)\end{array}$ & $\begin{array}{l}-4.9145^{\star} \\
(0.023)\end{array}$ & $\begin{array}{l}-4.6837^{\star} \\
(0.012)\end{array}$ & $\begin{array}{l}-4.2405^{\star} \\
(0.019)\end{array}$ \\
\hline Dom. health exp, ஃGDP & $\begin{array}{l}-2.4388 \\
(0.746)\end{array}$ & & & $\begin{array}{l}-18.6727 \star \\
(0.044)\end{array}$ & $\begin{array}{l}-23.9377 \star \star \\
(0.004)\end{array}$ & $\begin{array}{l}-15.1692 * \\
(0.036)\end{array}$ & $\begin{array}{l}-2.4265 \\
(0.809)\end{array}$ & & \\
\hline DAH Global Fund, ஃGDP & $\begin{array}{l}-23.485 \\
(0.575)\end{array}$ & & & $\begin{array}{l}-29.604 \\
(0.684)\end{array}$ & & & $\begin{array}{r}26.575 \\
(0.562)\end{array}$ & & \\
\hline 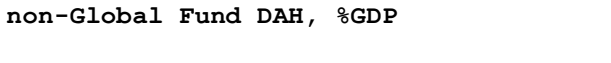 & $\begin{array}{r}16.520 \\
(0.357)\end{array}$ & & & $\begin{array}{r}19.411 \\
(0.383)\end{array}$ & & & $\begin{array}{r}33.605 \\
(0.091)\end{array}$ & $\begin{array}{l}32.887 \\
(0.064)\end{array}$ & $\begin{array}{l}25.096 \\
(0.105)\end{array}$ \\
\hline Prevalence, : & $\begin{array}{r}0.0987 \\
(0.241)\end{array}$ & $\begin{array}{r}0.0692 \\
(0.186)\end{array}$ & $\begin{array}{l}0.0708 \\
(0.174)\end{array}$ & $\begin{array}{l}-2.0654 \\
(0.103)\end{array}$ & $\begin{array}{l}-1.5355 \\
(0.129)\end{array}$ & $\begin{array}{l}-1.8402 \\
(0.075)\end{array}$ & $\begin{array}{l}0.0433 * \\
(0.015)\end{array}$ & $\begin{array}{l}0.0363 * \star \\
(0.007)\end{array}$ & $\begin{array}{l}0.0326^{\star} \\
(0.012)\end{array}$ \\
\hline Phase 1 change mortality, abs & $\begin{array}{l}-1.955 \\
(0.839)\end{array}$ & & & $\begin{array}{r}5.356 \\
(0.964)\end{array}$ & & & $\begin{array}{l}12.365 \\
(0.525)\end{array}$ & & \\
\hline TB treatment success, $\frac{\circ}{0}$ & & & & $\begin{array}{l}-0.0292 \\
(0.122)\end{array}$ & $\begin{array}{l}-0.0268 \\
(0.110)\end{array}$ & & & & \\
\hline
\end{tabular}


P1 change TB treatm success, abs

\begin{tabular}{|c|c|c|c|c|c|c|c|c|}
\hline & & & $\begin{array}{l}-0.0643 \\
(0.055)\end{array}$ & $\begin{array}{l}-0.0575 \\
(0.065)\end{array}$ & $\begin{array}{l}-0.0496 \\
(0.094)\end{array}$ & & & \\
\hline 0.9013 & 0.8656 & 0.9105 & 0.1501 & & & 1.2380 & 1.1918 & 1.1765 \\
\hline$(0.236)$ & $(0.117)$ & $(0.098)$ & $(0.846)$ & & & $(0.235)$ & $(0.177)$ & $(0.180)$ \\
\hline-0.0267 & & & 0.8678 & 0.4506 & $0.6300 *$ & 0.8941 & 0.9239 & 0.7869 \\
\hline$(0.956)$ & & & $(0.093)$ & $(0.188)$ & $(0.048)$ & $(0.162)$ & $(0.066)$ & $(0.095)$ \\
\hline 0.0457 & & & 0.6171 & & & 0.7601 & 0.8336 & 0.7489 \\
\hline$(0.937)$ & & & $(0.367)$ & & & $(0.274)$ & $(0.169)$ & $(0.193)$ \\
\hline-0.4462 & & & 0.6481 & & & 0.1229 & & \\
\hline$(0.462)$ & & & $(0.303)$ & & & $(0.897)$ & & \\
\hline 0.0300 & & & 1.7724 * & $1.8834 * * *$ & $1.9316 * \star *$ & -0.4364 & & \\
\hline$(0.968)$ & & & $(0.048)$ & $(0.000)$ & $(0.000)$ & $(0.618)$ & & \\
\hline 0.3710 & & & $2.8493 *$ & $2.6283 * *$ & $2.7359 * \star *$ & -1.9678 & $-1.5541 *$ & $-1.5000 *$ \\
\hline$(0.650)$ & & & $(0.011)$ & $(0.003)$ & $(0.001)$ & $(0.052)$ & $(0.021)$ & $(0.023)$ \\
\hline-0.0465 & & & -0.0903 & & & -2.3384 * & $-1.8188 * \star$ & $-1.7857 * \star$ \\
\hline$(0.957)$ & & & $(0.929)$ & & & $(0.023)$ & $(0.002)$ & $(0.002)$ \\
\hline-1.0304 & $-1.0356 *$ & $-1.0390 * \star$ & 1.5716 & $1.5566 *$ & $1.9631 * \star$ & -1.7939 & $-1.3018 *$ & $-1.1957 \star$ \\
\hline$(0.209)$ & $(0.011)$ & $(0.010)$ & $(0.109)$ & $(0.014)$ & $(0.002)$ & $(0.063)$ & $(0.010)$ & $(0.015)$ \\
\hline 0.2273 & & & -0.2961 & -0.6864 & & -0.9286 & -0.8736 & -0.7405 \\
\hline$(0.715)$ & & & $(0.682)$ & $(0.060)$ & & $(0.304)$ & $(0.068)$ & $(0.100)$ \\
\hline-0.5619 & & & -0.2592 & & & 0.0211 & & \\
\hline$(0.279)$ & & & $(0.682)$ & & & $(0.978)$ & & \\
\hline 0.1047 & & & -0.2782 & & & 0.1012 & & \\
\hline$(0.708)$ & & & $(0.400)$ & & & $(0.795)$ & & \\
\hline 0.093 & 0.078 & 0.077 & 0.096 & 0.083 & 0.073 & 0.124 & 0.119 & 0.115 \\
\hline 192 & 192 & 192 & 164 & 164 & 164 & 139 & 139 & 139 \\
\hline
\end{tabular}


On metrics and financing for the Sustainable Development Goals 


\section{Chapter 7}

\section{Synthesis}

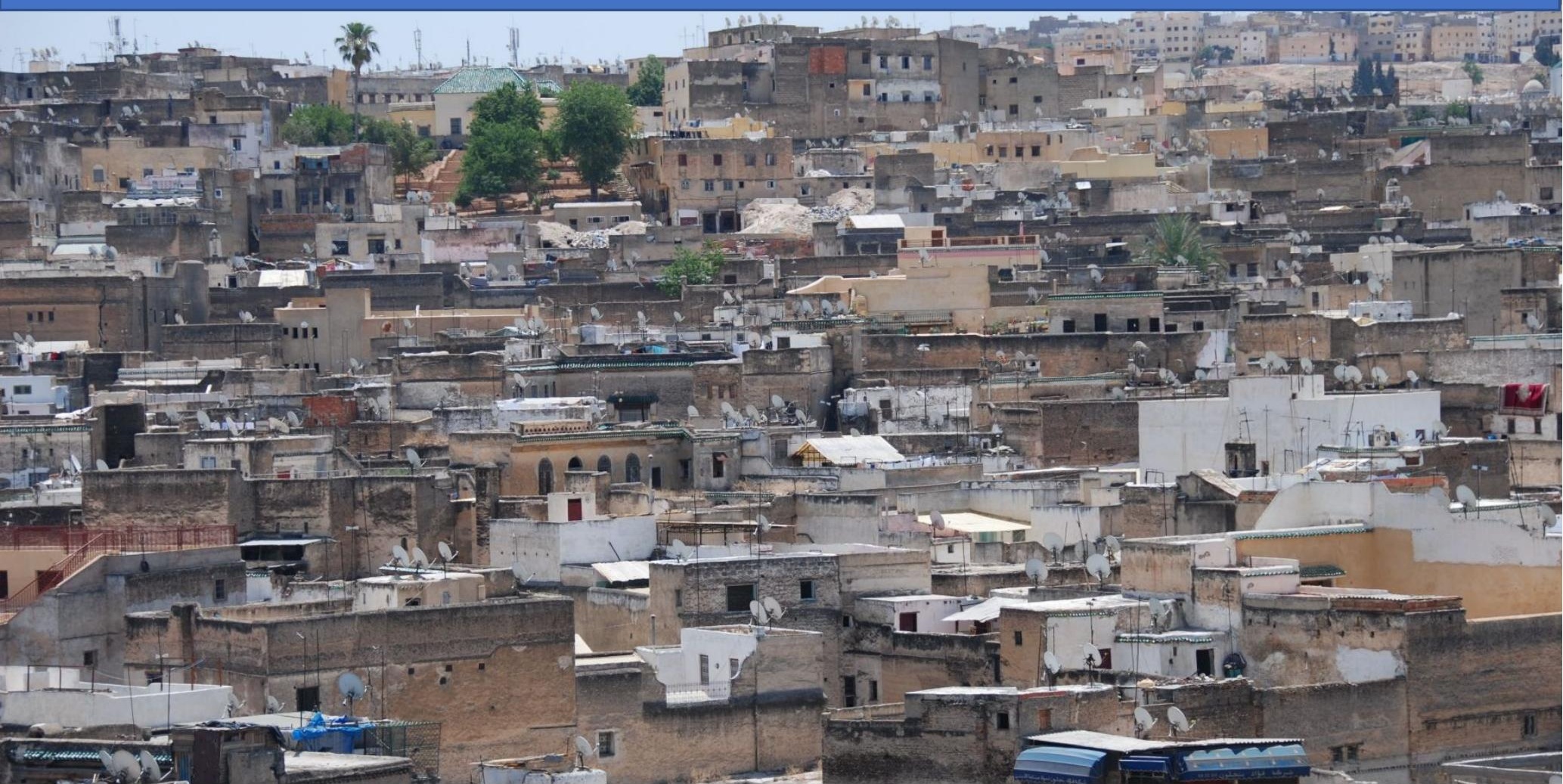




\subsection{Synthesis}

Countries around the world face deep economic, social, and/or environmental challenges that are expected to worsen under business as usual pathways (Sachs, 2015; van Vuuren et al., 2015). In response, member states of the United Nations have adopted the legally non-binding Sustainable Development Goals (SDGs) in 2015 (United Nations, 2015a). They build on the partial success of the Millennium Development Goals (MDGs), particularly in health (McArthur and Rasmussen, 2017). The SDGs focus on integrating development and climate change, a connection that was not made under the MDGs. Since the turn of the millennium, novel multilateral financing mechanisms have been launched to channel public resources towards specific sustainable development challenges. Among them, the Global Fund to Fight AIDS, Tuberculosis and Malaria ('Global Fund'), the Global Alliance for Vaccines and Immunization (Gavi), and the Green Climate Fund stand out as providing the largest volumes of concessional financing (OECD, 2016c).

This thesis considers key issues related to the monitoring and financing of the SDGs. Chapters 2-6 each address a specific research question, as outlined in Chapter 1. Our findings are presented in three groups that together cover some of the critical implementation challenges for the recently adopted SDGs.

The first group of findings relates to SDG metrics and country baselines (Chapter 2). We introduce a SDG Index, a new index that quantifies the distance countries must cover to achieve the SDGs. We demonstrate that even the wealthiest countries face major challenges in achieving the SDGs and that many countries' development models are imbalanced across the economic, social, and environmental dimensions of sustainable development. Further, we show that the SDG Index may generate novel insights into the determinants of subjective wellbeing, which has become a central objective of public policy in many countries.

Second, we estimate public and private financing needs for development under climate change, and to achieve the Sustainable Development Goals in low- and lower-middle income countries (Chapters 3 and 4 ). The thesis proposes a methodology and provides initial answers to the question of how the composition and scale of investment profiles need to change for an economy to achieve the time-bound SDGs, particularly in light of ongoing climate change. Results suggest that low- and some lower-middle-income countries will require significantly greater inflows of international private and public development financing to meet an estimated $\$ 1.4$ trillion in incremental annual investment needs through to 2030 . We estimate that global incremental investment needs amount to $\$ 2.3$ trillion per year. These incremental investment needs appear manageable at $1.5-2.5 \%$ of average annual world GDP through to 2030 with global savings currently estimated at $\$ 22$ trillion (ICESDF, 2014). Moreover, we find that incremental investment needs for the SDGs are far lower than suggested by other authors (UNTT, 2013), likely due in part to a granular assessment and removal of overlaps across SDG investment areas.

While higher levels of investment, including more development assistance, are needed (Chapters 3 and 4), the availability of more finance alone will not achieve the SDGs. The development economics literature is replete with examples where aid has failed to achieve the intended effects owing to project design, implementation, the overall policy environment, and other causes (Temple, 2010).

Next, we therefore consider the institutional design for delivering international development assistance by studying the impact of the Global Fund's design principles, including the Technical Review Panel (TRP), for the fight against HIVIAIDS, TB, and 
Malaria. Our investigation into health financing is motivated by the fact that the health sector has performed well under the MDGs (Kassebaum et al., 2014; McArthur and Rasmussen, 2017), driven significantly through increased investments supported by the Global Fund (Chapter 5). The findings in this thesis suggest that the Global Fund with its unique design principles and independent evaluation of proposals (Chapters 5 and 6) may serve as a model for scaling up development assistance in other SDG investment areas, though some areas may require different institutional designs. Our findings help inform policy debates on the most appropriate institutional forms for generating quality demand for development finance, directing scarce resources to the countries most in need, and ensuring effective implementation to achieve the intended goals.

The remainder of this summary chapter is structured as follows: Section 7.2 seeks to present answers to each of the five research questions developed in Chapter 1. Section 7.3 critically evaluates the methods and data used to derive the answers to each question. In Section 7.4 we consider the limitations of the present analysis and outline suggestions for future research. Section 7.5 concludes by synthesizing the policy implications of the scientific contributions made in this thesis.

\subsection{Answers to research questions}

\subsubsection{Baselines for the SDGs}

In Chapter 2, based on Schmidt-Traub et al. (2017), we consider:

Research Question 1: How to identify SDG implementation priorities for each country based on a measurement of countries' distance from achieving the goals?

To answer this question, we develop and present a new composite index and dashboards for the SDGs that normalize and integrate internationally comparable data using methodologies recommended by Booysen (2002), OECD and JRC (2016). Paying careful attention to data availability and international comparability, we collect and synthesize data from official and unofficial sources to generate the first country-level baselines for the SDGs that identify implementation priorities for each country. Indicators are included if data are available for at least $80 \%$ of all UN members countries with a population greater than 1 million. All data are censored at 2.5 percentile to remove extreme values. They are normalized between a lower bound indicating worst performance in the sample of countries and an upper bound indicating optimum performance for that indicator. Data are aggregated arithmetically within each SDG and across the 17 goals. In this way, the SDG Index measures countries' distance from achieving each SDG and the 17 goals as a whole. Alternative specifications of the SDG Index are tested to confirm robustness of the results. Countries are included in the SDG Index if they have data available for at least $80 \%$ of the indicators used in the Index. We then investigate how countries' performance on the SDG Index and Dashboards compares with other commonly used metrics for development. We close Chapter 2 by considering data gaps and by applying the SDG Index as a cross-country predictor of subjective well-being, a key aggregate objective of public policy (Stiglitz et al., 2009; OECD, 2013; Helliwell et al., 2016b).

We find that a global SDG Index can be constructed using 63 indicators (Table 8, page 57) drawn from official and unofficial sources with due attention paid to data quality. The official SDG indicators (IAEG-SDGs, 2016a) are not sufficient in terms of data availability and scope to describe the full set of SDGs. The SDG Index is harmonized 
across countries to permit comparisons, and it is reasonably robust to alternative specifications. In this first version of the SDG Index we are able to include 149 out of 193 member states of the United Nations. The 44 countries not included in the index (Table 12 , page 65) are mostly countries with very small populations that lack the administrative capacity and/or sample size to collect key SDG variables, or countries experiencing or just emerging from conflict. Reducing the number of countries not included in the SDG Index should be a priority for future research and policy. An augmented index for OECD countries reports an additional 14 indicators for the member countries of the OECD.

The SDG Index provides a novel tool for establishing country baselines and for determining the gap that each country must close to achieve the SDGs. It also serves to compare performance across countries and goals. The thresholds and methodology for normalizing the data and scoring countries on a scale from 0 to 100 are transparent and can be refined through further scientific investigations and improved data.

Significant data gaps remain in establishing baselines for the SDGs, as summarized in Table 5 (page 41) and described further in Section 7.4 below. Also, the SDG Index does not constitute a structural regression model for the SDGs. Partly as a result, several indicators, such as wasting and stunting, are highly correlated. Finally, the SDG Index draws on data from different time periods and includes a large number of missing variables. We consider these methodological issues in greater detail in Section 7.3.

Overall, the SDG Index is highly correlated with the natural log of GDP per capita (PPP) and the Human Development Index (HDI). Correlations with the Global Competitiveness Index and the Environmental Performance Index are slightly weaker. They are lowest for the Index of Economic Freedom and the Global Peace Index, though both indices are positively associated with the SDG Index. This suggests that on balance, richer countries perform better on the SDGs, even though they face serious shortfalls, as demonstrated by the SDG Dashboards (Figure 1, page 33). The challenges vary from country to country, but most high-income countries score relatively poorly in addressing climate change, lowering inequalities, halting the loss of biodiversity, and contributing their fair share towards the global partnership to achieve the SDGs. Some high-income countries have low scores on gender equality. In order to achieve the SDGs, poorer countries need to make substantial progress across all goals with a focus on ending extreme poverty, ensuring access to essential services and infrastructure, curbing environmental degradation, and promoting social inclusion.

Substantial differences exist in performance within and across regions or income groups. For example, the United States ranks $9^{\text {th }}$ in per capita GDP (PPP) (IMF, 2016) but $25^{\text {th }}$ in the SDG Index, owing to poor performance on SDGs tracking environmental sustainability, social inclusion, peace and justice. Findings are similar for China, Russia, and the UK. Some countries, notably from the Middle East and North Africa, perform well on meeting basic needs, as measured by the HDI, but fall short on the SDG Index owing to high gender and income inequalities, and environmental degradation. On balance, the SDG Index and Dashboards show which countries' development is imbalanced across the three dimensions of sustainable development. They provide useful tools for benchmarking country performance and for conducting scientific investigations into cross-country differences.

The SDG Index is partially correlated with subjective well-being (Table 4, page 39) in the presence of per capita GDP and unemployment, common correlates considered in the literature (Clark and Oswald, 1994; Dolan et al., 2008; Delhey and Kroll, 2013). This finding illustrates the usefulness of the SDG Index in understanding determinants 
of and cross-country differences in subjective well-being and other policy objectives. It opens opportunities for other scientific inquiries into the SDGs.

This first version of the SDG Index and Dashboards cannot be used to estimate trends in countries' progress towards the SDGs because several indicators only have one recent data point. Moreover, data for indicators are reported with different periodicities, so year-on-year changes in the SDG Index do not represent changes in progress towards all SDG indicators. Alternative tools will be needed to report year-on-year changes and trends towards achieving the goals.

\subsubsection{Investment needs for climate-resilient development}

In Chapter 3, based on Fankhauser and Schmidt-Traub (2011), we consider:

Research Question 2: How can interventions and associated financing needs for development and climate change adaptation be integrated to estimate the combined financing needs in Africa?

Integrating climate change adaptation measures into strategies for achieving the narrow development objectives described in the Millennium Development Goals (MDGs) requires greater resources than has been reported in the literature to date. We find that including climate change adaptation raises external resource needs for development, as estimated by the MDG Africa Steering Group (Ki-Moon et al., 2008), by $40 \%$ to $\$ 100$ billion per year through to 2020 . The increment comprises some $\$ 10$ billion per year for development interventions omitted by Steering Group and some \$10-21 billion in external public finance needs for climate change adaptation. The sectors with the greatest additional financing needs for climate change adaptation are infrastructure, agriculture, disaster response, and health (Table 18, page 86).

The mark-up for adaptation measures is significantly higher than reported in the scientific literature (Boko et al., 2007; Stern, 2007; Fankhauser, 2010; Barr et al., 2010). We identify several reasons for this discrepancy. First, Chapter 3 considers a broader range of interventions than included in most estimates of investment needs for climate change adaptation (Table 19, page 87). Second, the literature on adaptation tends to apply mark-ups for climate-proofed interventions (such as better design standards for roads that resist higher temperatures and more frequent high-precipitation events) to baseline expenditure. Yet, baseline levels of expenditure may be far below investments needed to achieve development objectives, such as the MDGs. Therefore, we apply the mark-up for climate-proofing infrastructure and other investments to the aspirational investment needs identified by the MDG Africa Steering Group (Ki-Moon et al., 2008).

The findings in Chapter 3 demonstrate the complexity and importance of integrating investment needs assessments for climate change adaptation and development interventions. The estimates of resource needs reported in the chapter are indicative and imperfect in many ways. They draw heavily on existing top-down analyses of adaptation and MDG resource needs that are aggregated and subject to substantial margins of error. The reliance on existing cost data also creates some inconsistencies in the time frame and other assumptions that underpin the original estimates. Owing to data gaps, the results may significantly underestimate investment needs for "climate-resilient development' in Africa. This applies in particular to the period after 2020 when substantially higher incremental resource needs can be expected for climate change adaptation owing to the acceleration of climate change even under the assumption of aggressive reductions in greenhouse gas emissions (IPCC, 2014). 
Despite these shortcomings, the methodology introduced in the chapter represents a significant improvement over previous investigations into the economics of climateresilient development. It provides a first integrated assessment of investment gaps for achieving 'development' and 'climate change adaptation' objectives, while highlighting key knowledge gaps and provides an analytical framework for improving the quantitative understanding of investment needs.

The methodology also provides a framework for implementing the requisite measures as part of an integrated development program. Like the original MDG estimates, our analysis is organized along sectoral lines that correspond roughly to the organizational structure of most governments, so that investment needs can be linked more easily to explicit sector objectives and delivery mechanisms.

By establishing a baseline for required investments in 'development' the methodology also provides an approach for defining and quantifying the 'additionality' of resources for climate change, which has been a central policy question in the climate economics literature and international climate agreements (Stern, 2007; World Bank, 2010c; UNFCCC, 2007, 2015). According to this approach, resources for adaptation are deemed 'additional' if they are provided above and beyond the MDG financing summarized in Table 18 (page 86).

\subsubsection{Investment Needs to Achieve the SDGs}

In Chapter 4, based on Schmidt-Traub (2015), we considered:

Research Question 3: What are the private and public financing needs from domestic and international sources to achieve the SDGs in low- and lower-middle-income countries?

The chapter groups SDG investments into eight 'SDG investment areas': (1) health; (2) education; (3) social protection; (4) food security and sustainable agriculture; (5) infrastructure, including (5.1) energy access and low-carbon energy infrastructure; (5.2) water and sanitation; (5.3) transport infrastructure; (5.4) telecommunications infrastructure; (6) ecosystem services and biodiversity; (7) data for the SDGs; and (8) emergency response and humanitarian work. These SDG investment areas cover the range of public and private investments needed to achieve the 17 SDGs with the exception of fully commercial business investments that fall outside the scope of our analysis.

Drawing on our preliminary suitability score for assessing the quality and robustness of each needs assessment (Table 25, page 105), we find that needs assessments in the social sectors (health and education), access to water and sanitation, and access to energy services tend to be strongest, while needs assessments for the environment, large-scale infrastructure, agriculture, and food security are weakest. Investment needs for social protection remain to be estimated, as do commercial business investments needed to achieve economic growth targets. The framework also underscores that many SDG needs assessments do not integrate climate change adaptation and mitigation.

To make available needs assessments comparable and to aggregate them into an estimate of overall SDG investment needs, we perform several types of adjustments (Table 27, page 120). All estimates are rebased to 2013 US\$ at international market rates. We estimate the private share of investments drawing on UNCTAD (2014) and other sources, and differentiate between aggregate investment needs in low- and 
lower-middle-income countries. Overlaps between different needs assessments are identified and removed. Similarly, we identify gaps in resource estimates and seek to fill them using other studies. Remaining gaps are specified in the summary assessments. Finally, where appropriate we adjust resource needs estimates for the cost of climate change mitigation and adaptation, drawing on the findings in Chapter 3 and other sources.

After these adjustments, incremental SDG financing needs are estimated at $\$ 1.4$ trillion (in 2013 US\$) per year in low- and lower-middle-income countries (Table 26, page 114). Based on an extrapolation of recent GDP growth rates, incremental investments for the SDGs might correspond to some $4.0-11.5 \%$ of GDP in these countries (18-45\% in LICs and $3-9 \%$ in LMICs) or $0.8-1.3 \%$ of annual world GDP over the period. Annual investment needs in the SDGs rise to $5-16 \%$ of projected GDP in LICs and LMICs (25$65 \%$ in LICs; $4-12 \%$ in LMICs; $1.0-1.5 \%$ of world GDP) if GDP growth rates are half as high as projected. If growth accelerates beyond the projected rates then the share of GDP that must be invested to achieve the SDGs falls accordingly.

Less than half of these investments needs (39-45\%) can be financed commercially by the private sector. Opportunities for private financing are greatest for infrastructure and lowest for social services. They also rise as countries' per capita incomes increase. Note, that a lower share of private financing does not preclude the private provision of SDG investments based on public financing.

Based on a stylized financing analysis, we project an average annual financing gap of \$152-163 billion between 2015 to 2030 in low-income countries. Lower-middle-income countries are projected to cover their average investment needs drawing on domestic public and private international capital, including non-concessional loans. However, these countries may require international co-financing at the beginning of the SDG period, when investments are likely to rise faster than countries' ability to mobilize private and domestic public resources. If members of the OECD Development Assistance Committee increase their official development assistance to $0.7 \%$ of their GNI by 2030 , as suggested in the Addis Ababa Action Agenda (United Nations, 2015b), and if other high-income countries follow their example additional concessional international financing would far exceed the SDG financing gaps in low- and lower-middle-income countries.

\subsubsection{Lessons from the Global Fund for the SDGs}

In Chapter 5, based on Sachs and Schmidt-Traub (2017), we consider:

Research Question 4: To what extent do the key design principles of the Global Fund explain the institution's success in delivering $\$ 35$ billion in incremental health financing for novel and complex programs including in poorly governed countries and countries emerging from conflict?

Eight key design principles define the Global Fund's operating model and set it apart from other multilateral financing mechanisms: (i) country-led proposals; (ii) multi-stakeholder proposal design and implementation; (iii) independent, transparent, technical review and evaluation; (iv) political independence in grant allocations; (v) needs-based pooled financing; (vi) disease-specific funding implemented in broader health systems; (vii) performance-based funding; and (viii) financing mechanism without a stake in program design or implementation. These design principles helped the Global Fund generate a large volume of high-quality, country-led funding proposals that performed well across a broad range of operating environments and contributed to health-system 
strengthening. In this way, the Global Fund offers a novel and innovative approach to solving the aid allocation problem discussed widely in the development economics literature (World Bank, 1998; Collier and Dollar, 2002; Van de Walle, 2005; Wood, 2008). It appears to have succeeded in rapidly increasing the volume and quality of country funding proposals, an issue we will consider in greater detail below in discussing findings from Chapter 6.

The multi-stakeholder Country Coordination Mechanisms enhanced civil-society participation in program design and implementation. They also promoted innovation and the propagation of best practice across countries. The Global Fund was able to attract large volumes of unrestricted grant funding from all major donors, including ones that criticized the Global Fund's model at its inception. Overall, performance-based funding and the light-touch business model of the Global Fund minimized transaction costs and limited the misuse of funds. During the first 14 years of its existence, the Global Fund experienced operational challenges that were identified through independent evaluation and then addressed through gradual improvements in the organization's business model. Overall, the eight design principles appear to explain the institution's success in delivering $\$ 35$ billion in incremental health financing for novel and complex programs, including in poorly governed countries and countries emerging from conflict.

The successful track record of the Global Fund in a broad range of developing country environments, positions the organization well to serve as the primary funding vehicle to implement strategies to end the three epidemics (90-90-90 Strategy for HIV/ AIDS, the Global Plan to End TB, Global Malaria Action Plan) and to ensure universal health coverage (SDG Target 3.3). This would require broadening the Global Fund's business model to increase investments and implementation research for health system strengthening (Target 3.8), reducing preventable deaths (Targets 3.1 and 3.2), access to sexual and reproductive health services (Target 3.7), responsiveness to disease outbreaks such as Ebola (Target 3.d), and tackling non-communicable diseases (Target 3.4).

Given the success of the Global Fund, it is instructive to consider to what extent its design principles can be applied to other SDG investment areas that require large volumes of grant financing to scale up proven interventions in country-led programs (Chapter 4). The Global Fund mobilized funding for national programs that scaled-up known interventions and successfully addressed implementation challenges in the process. This might suggest that the Global Fund experience is particularly relevant for non-health SDG priorities that present similar investment challenges and experience high donor fragmentation (Acharya et al., 2006; Easterly, 2007). Among them, education stands out as likely most closely related to health: Both sectors rely on publicly funded national systems that provide standardized interventions to the population in a decentralized manner. The education sector also exhibits high donor fragmentation (Steer and Smith, 2015). Indeed, the similarities between education today and health in the early 2000 s are so close that the education sector often references health as a model for its own scaling up (UNESCO, 2013, 2015b). In particular, analysts emphasize the low quality of many national education programs (UNESCO, 2013), which mirrors the situation in the health sector at the turn of the century (Lu et al., 2010).

Other areas where reasonably well-known interventions need to be scaled up in national programs are smallholder farming (SDG 2), improved nutrition (SDGs 2 and 3), access to safe water supply and sanitation (SDG 6), and distributed rural electrification programs (SDG 7). Funding mechanisms targeting these areas may benefit from studying the Global Fund experience and considering the application of its design principles (for more details, see Section 7.5 below). 
On the other hand, the Global Fund model appears less suited for investment areas that require more market- and project-based financing solutions. This includes largescale infrastructure, the decarbonization of energy systems, and integrated urban development programs. Considering extent to which the Global Fund's key design principles can help inform financing mechanisms in these areas would require more analytical work.

Chapter 5 shows that the Global Fund has had a large effect on the health sector in developing countries. Pooled global financing mechanisms are important, but they are not sufficient to accelerate progress towards global goals. Success also requires proven and well-documented interventions, careful implementation research, effective institutions, political leadership, and many other dimensions discussed in the aid literature (Temple, 2010) and reviewed by Schmidt-Traub and Sachs (2015).

\subsubsection{Reconciling country ownership with independent technical ap- praisal}

In Chapter 6 we consider:

Research Question 5: Did the Global Fund's Technical Review Panel (TRP) make funding recommendations that were in line with the objective of allocating funding to countries most in need?

During the rounds-based mechanism (2002-2010) per capita funding requests to the Global Fund were correlated with disease prevalence rates. The volume of funding requests increased over time in the presence of sharp falls in the cost of interventions, demonstrating a significant scaling up of programs over time. However, per capita funding requests from countries with larger populations were financially suppressed: After controlling for other parameters, countries with larger populations requested less funding per capita, which challenges a central argument in support of the Global Fund's New Funding Model (HLIRP, 2011) and suggests significant unmet funding needs, particularly in countries with larger populations.

Throughout the rounds-based mechanism, the TRP fulfilled its role. It recommended proposals without regard to funding volumes, population size, or other country characteristics typically associated with different aid volumes, such as governance, domestic health expenditure, and DAH. Funding was directed towards higher-burden countries with lower incomes, consistent with the guiding principles of the Global Fund (GFATM, 2001), as analyzed in Chapter 5 . We find that, after controlling for other parameters, countries with large populations and/or high per capita funding requests, did not reduce the volume of funding after the TRP had rejected a proposal, but encouraged the country to reapply with a revised proposal (i.e. the proposal was rated 3 by the TRP). This suggests that countries did not believe the TRP exercised financial suppression in its consideration of proposals or else they would have lowered their funding requests to increase the likelihood of a positive recommendation.

The Global Fund, working with its Technical Partners, was effective at overcoming lower capacity to design and implement programs in poorer and/or fragile countries. After controlling for other regressors, fragile countries requested funding volumes that were not significantly different from those requested by other countries; they were as likely to have their funding requests approved by the TRP; and their grants performed equally well except for HIVIAIDS grants. Indeed, the TRP flagged concerns about the low quality of technical assistance for HIVIAIDS grants (TRP, 2009), which might partly explain the differential performance of fragile countries here. 
Higher funding requests and better ratings of proposals from PEPFAR countries suggest that countries that receive greater support in strengthening and scaling-up their response to HIVIAIDS can attract more funding from the Global Fund. This in turn suggests the presence of large unmet funding needs in non-PEPFAR countries, which expanded technical assistance might convert into high-quality proposals.

The Global Fund's success in operating across the full spectrum of country environments, including fragile and poorly governed countries, sets an example for financing health systems and other investment priorities under the SDGs. For example, the education sector currently operates separate pooled funding mechanisms for non-fragile and fragile countries. Given the need to lower transaction costs and the continuous transition from fragile to non-fragile environments, it would appear preferable to operate one integrated pooled financing mechanism for education. Such a mechanism would promote learning and the propagation of operational lessons across all countries.

English-speaking countries obtained higher volumes of funding for malaria and TB. TRP reports frequently referred to quality problems with the translation of proposals and supporting documents into English. The Global Fund and other mechanisms must ensure that language does not become a barrier to accessing financing.

Findings from our regressions support the conclusion that the Global Fund promoted substantial learning and improvements in the quality of country proposals. This finding is notable since the development economics literature often concludes that rates of learning are low in development cooperation (Berg, 2001; Easterly, 2007; van Kerkhoff and Szlezák, 2010; Temple, 2010). It is likely that the TRP's transparent rating of proposals, the release of findings from each funding round, and the systematic review of lessons learnt with Technical Partners (e.g. WHO, UNAIDS, Stop TB, and Roll-Back Malaria) contributed to propagating knowledge on scaling up interventions across countries. This tentative conclusion is supported by the experience of the Technical Partners (e.g. WHO (2015a)). The country-led funding model encouraged scaling-up since countries were not constrained in the volume of per capita funding they could request, and success in one country inspired others to follow (Shakow, 2006; van Kerkhoff and Szlezák, 2010; HLIRP, 2011).

Taken together, the evidence demonstrates that a demand-based funding mechanism relying on independent technical review of proposals without ex-ante country allocations can generate needs-based funding allocations. It can stimulate quality demand even in poor and fragile countries contrary to widespread expectations in 2002 (Chapter 5). In conclusion, the TRP has played the role it was expected to and provides a novel mechanism for addressing the tension between country autonomy or country ownership and conditionality in ensuring sound use of resources (see in particular OECD (2008), Temple (2010), and Chandy and Kharas (2011)). To our knowledge Chapter 6 represents the first systematic analysis of the working of the TRP. It may therefore hold important lessons for financing other SDG investment areas described in Chapter 4.

With the exception of Gavi no other multilateral financing mechanism reviewed in the 2016 Multilateral Development Review undertaken by the UK government (DFID, 2016) has a fully independent technical review panel along the lines of the Global Fund's TRP that makes funding recommendations to the Board, which the latter can only accept or reject. It would therefore appear instructive that other multilateral financing mechanisms, such as the Global Environment Facility, the Green Climate Fund, 
the Global Partnership for Education, or the International Fund for Agricultural Development, study lessons from the Global Fund's TRP to ascertain their potential relevance for improving the performance of these financing mechanisms.

\subsection{Evaluation of methodologies and data}

In this section, we discuss the strengths and weaknesses of the methodologies employed in this thesis to answer the five research questions.

In Chapter 2 we construct a novel SDG Index and Dashboards using methodologies recommended by Booysen (2002) and OECD and JRC (2016). Other investigations into the SDGs have since employed approaches that are very similar to ours (OECD, 2016a). As is common with composite indices (OECD and JRC, 2016), the weighting of indicators and goals is subjective. Our equal weighting of each goal appears most closely aligned with the intention of the framers of the SDGs (United Nations, 2015a), but will be challenged by some researchers who consider some goals as more foundational than others (Lu et al., 2015; Allen et al., 2016; Nilsson et al., 2016; Sridhar, 2016). Some authors propose using variable weightings to account for individual or country priorities within a broad set of goals (OECD Better Life Initiative, 2011; Lind, 2014; OECD and JRC, 2016). However, since our aim is to compare country performance, a common weighting and aggregation technique is necessary. This also has the benefit of avoiding 'cherry picking' by countries or researchers (Dasgupta and Mäler, 2000) and sharpens the focus on an integrated agenda to achieve the SDGs.

The data are not normally distributed, and the distributions of some variables depart far from the normality assumption (Table 10, page 61). For this reason, and to ensure normalization of each variable that does not vary over time as countries progress towards the SDGs, we cannot use z-scores to construct the index. Instead we perform a linear transformation to a scale from 0 to 100. Lower bounds are defined by worst performance measured at 2.5 percentile. They will be time invariant assuming that countries progress towards the goals. Upper bounds are defined by the SDG Targets. Where the targets do not yield quantitative thresholds, upper bounds are set by the average of the five best-performing countries (Table 11, page 63). As a result, some countries already exceed the upper bound for some variables, and more may do so as average performance improves over time. Since the purpose of the SDGs is to spur countries to close major gaps across the three dimensions of sustainable development, exceeding the upper bound by some countries does not significantly limit the utility of the SDG Index.

Sensitivity analyses by scaling method (Table 13, page 66) suggest that the SDG Index scores and rankings are reasonably robust to different methods for setting the upper and lower bounds. Similarly, they are robust to different arithmetic and geometric aggregation techniques (Table 13, page 66).

The indicators constituting the SDG Index were chosen to ensure maximum coherence with the official SDG Indicators (IAEG-SDGs, 2016a), the SDGs, and their targets. As a result, several indicators are highly correlated. Examples include wasting and stunting, or the high correlation between extreme headcount poverty and social outcome indicators. The SDG Index should therefore not be confused with a structural regression model of the SDGs. Such a model would need to consider a reduced set of indicators and be corrected for statistical issues, including collinearity, non-normality of residuals, and heteroscedasticity. For the purposes of monitoring the SDGs and identifying priority implementation challenges, these statistical questions are not a priority consideration. The equal weighting of each SDG within the SDG Index further reduces 
the impact of correlation across indicators, since such correlations are highest within goals (Nilsson et al., 2016).

To test for reduced specifications of the indicator set, we conducted principal component analyses (PCAs) of the indicators included in the SDG Index (not reported in Chapter 2). The PCAs generated a large number of components that did not add any additional insights. It would be interesting to pursue PCAs with future editions of the SDG Index.

A further methodological challenge is that some indicators included in the SDG Index describe outcome variables, such as child mortality, while others consider input or output variables, such as the number of physicians per capita or the carbon intensity of power generation. The inclusion of outcome and input/output metrics for monitoring the SDGs raises methodological issues (Booysen, 2002; OECD and JRC, 2016) that deserve further scrutiny. On the other hand, there are good reasons for using a range of intermediate and outcome measures to help turn the SDGs into scorecards and management tools (SDSN, 2015a) and support their implementation (SDSN, 2013; UN Statistics Division, 2017).

Since we do not model missing data for the SDG Index, the analysis relies on data available for different time periods (see Table 8, column 5, page 57). This may distort data for countries that have experienced significant changes since the data were collected. In view of the sparsity of data and the resulting difficulties involved in modeling missing data discussed in Section 7.2.1, the only reliable way to address this shortcoming is to collect data more regularly.

Data gaps constitute a major limitation for the SDG Index in its current form, including the lack of scientifically robust indicators for several SDGs (Table 5, page 41), the low frequency of data collection, and the lack of integration with other forms of big data. To close these gaps as far as possible we have considered all available data sources including science-based indicators that are not included among the official SDG indicators (IAEG-SDGs, 2016b). Data gaps are largest for the environmental impact of food production systems (SDG 2), internationally comparable data on education outcomes (SDG 4), economic empowerment of and violence against women (SDG 5), decent work (SDG 8), sustainable consumption and production (SDG 12), and the management of terrestrial and marine ecosystems (SDGs 14 and 15). In particular, we lack data on international spill-over effects of one country's action on the ability of other countries to achieve the SDGs. These data gaps make the SDG Index incomplete and may introduce a bias in the results.

Other authors (OECD, 2017b) restrict their assessment of SDG performance to the official SDG indicators for which adequate data are available. This approach is politically justified, but yields results that are difficult to reconcile with the evidence. For example, assessing OECD performance using only data for official SDG indicators suggests that gender equality is the greatest SDG challenge in these countries, while SDG 14 (marine ecosystems) has been largely achieved, and SDG 13 (climate change) represents relatively modest challenges. These findings are at odds with the scientific literature on oceans (Halpern et al., 2012; Inniss et al., 2016) and climate change (IPCC, 2014; Bataille et al., 2016; Sachs et al., 2016c).

The limited data available for some indicators and differences in the periodicity of data collection make it impossible to estimate trend data in the current form of the SDG Index. This contrasts with the approach chosen by the Global Burden of Disease Collaboration (2016) who model missing data for the health SDGs using data and statistical tools reported by Wang et al. (2016). Yet, data quality and availability for many non- 
health SDGs are lower, so this approach cannot be used to estimate trends for the SDG Index.

We conclude that the methodology employed in Chapter 2 generates first answers to Research Question 1. It can be applied to all countries, and be tailored to sub-groups of countries, as illustrated with the augmented SDG Index for OECD countries. Our findings point to substantial weaknesses in the underlying data that have also been highlighted by others (ECOSOC, 2015; Espey et al., 2015; SDSN, 2015a; Selomane et al., 2015).

In Chapter 3 we draw on the most comprehensive assessment of MDG financing needs that has been validated by all multilateral development agencies working in Africa (Ki-Moon et al., 2008). We could not identify any peer-reviewed needs assessments in the scientific literature. We then added incremental resource needs for adaptation measures drawn from a range of different studies.

Our estimates provide the first integrated assessment of investment needs for climateresilient development or 'development in a hostile climate' (Stern, 2009a) that has so far been cited in 249 publications listed on Google Scholar (accessed on 10 July 2017). Yet as discussed in Chapter 3 , the results are partial since they exclude climate change mitigation measures, ecosystem services, sustainable consumption, and other SDG priorities. Moreover, the results draw on existing top-down analyses of adaptation and MDG resource needs that are, by necessity, aggregated, have substantial margins of error, and suffer from analytical shortcomings (Parry et al., 2009; Fankhauser, 2010), which our methodology does not resolve fully. Due to differences in methodologies, the reliance on published cost data also creates some inconsistencies in the time frame and other assumptions that underpin the results.

Moreover, the analysis does not employ a general equilibrium framework. Therefore the evolution of labor and other factor prices as well as the real exchange rate will require separate analyses that combine intervention-based needs assessments with computable general equilibrium models (Bourguignon et al., 2008; Schmidt-Traub, 2015). Despite these shortcomings, the methodology provides an analytical framework for integrating development and adaptation needs into a coherent framework that is consistent with the sectoral organization of governments. It identifies research gaps to be filled by subsequent studies and discussed further below.

Chapter 4 synthesizes the available literature on investment needs to achieve the SDGs in low- and lower-middle-income countries. The analysis presents the first assessment of investment needs for the SDGs. It improves on earlier assessments of incremental investment needs for sustainable development (e.g. UNTT (2013) and UNCTAD (2014)) by (i) distinguishing between low- and lower-middle-income countries and presenting an analytical framework that can be scaled to national, regional, and global levels; (ii) considering opportunities for public and private investments, (iii) assessing the suitability of underlying sector needs assessments and harmonizing results across studies to increase their comparability, remove overlaps, and fill gaps; (iv) including incremental investment needs for climate change adaptation and mitigation, drawing on the findings from Chapter 3 , and (v) outlining a stylized financing analysis for incremental investment needs. Chapter 4 also considers a much wider range of the literature than previous studies.

We find that few sectors have peer-reviewed assessments of investment needs to meet the SDGs. The chapter must therefore rely largely on the policy and 'grey literature', which reduces the robustness of the results. Unfortunately, investigations into some of the SDG investment areas that require the greatest incremental resources, 
such as infrastructure, also tend to have the lowest 'suitability score', so their findings are likely to have a high margin of error.

Another methodological challenge is that few available resource needs assessments provide a comprehensive analysis that includes all interventions under an SDG investment area and incorporates climate change mitigation and adaptation. Few studies also specify the share of expenditure amenable for private financing, and results are not always available for low- and lower-middle-income countries. We therefore make adjustments to the studies (Table 25, page 105), often in consultation with the original authors, so that results can be reported in Table 26 (page 114). The analysis developed in Chapter 4 identifies research and knowledge gaps and provides an analytical framework for future sector needs assessments.

We could not find a general equilibrium model that addresses the full range of SDG investment areas. Models developed for the SDGs (Agénor et al., 2005; Bourguignon et al., 2008; IMF, 2008) cover only a small share of the SDG investment areas. As a result, our analysis in Chapter 4 does not address economy-wide effects. From investigations into the MDGs (e.g. Bourguignon et al. (2008)) we know that the large increases in public and private investments required to achieve the SDGs will likely have significant effects on macroeconomic variables, including demand for skilled and unskilled labor, real exchange rates, consumption, and the composition of investment. General equilibrium assessments of the SDGs and their financing should therefore be pursued as a priority.

The stylized financing analysis for the SDGs is highly sensitive to projections of GDP. Lower GDP growth rates would reduce domestic resource mobilization and thereby increase the external financing gap. A more sophisticated financing analysis is needed to, inter alia, consider domestic resource mobilization in greater detail; include debt financing; distinguish between concessional and non-concessional international public finance; endogenize economic growth assumptions; and consider the microeconomic implications of increased domestic resource mobilization on the achievement of the SDGs.

In Chapter $\mathbf{5}$ we assess the Global Fund's performance against five initial criticisms drawing on eight key design principles identified in this chapter. The strength of the methodology lies in synthesizing for the first time a broad spectrum of the policy and academic research literature describing and assessing the Global Fund. But therein also lies a weakness since the methodology cannot assess ways in which the Global Fund has uncovered and then addressed weaknesses in its business model. In particular, the chapter cannot elucidate to what extent the Global Fund's shift to the New Funding Model reflected a reaction to the growing maturity and sophistication of countries' disease strategies or whether it was principally driven by donors' concerns to limit the scaling up of Global Fund resources. Moreover, detailed econometric assessments of the Technical Review Panel are needed to better understand how the Global Fund generated quality demand from developing countries, a question we addressed in Chapter 6.

Chapter 5 also identifies lessons from the Global Fund's design principles for the financing of other health and non-health SDG priorities. However, we have not conducted an in-depth assessment of the design mechanisms of other major multilateral mechanisms, including the Global Environment Facility, the Global Partnership for Education, and the Green Climate Fund. For this reason, the conclusions relating to the operation of these mechanisms remain preliminary and require further scrutiny. 
Finally, in Chapter 6 we generate a novel dataset combining data on funding proposals submitted to the TRP provided by the Global Fund secretariat; TRP recommendations and Board decisions; grants and their performance; and consistent country characteristics that significantly expand the range of predictors considered by previous investigations into the performance of Global Fund grants (Lu et al., 2006; Katz et al., 2010; Radelet and Siddiqi, 2007; Fan et al., 2013; Bowser et al., 2014). We then adapt these published methods to generate four sets of regression models that are run separately for each of the three major Global Fund disease categories, consistent with earlier studies (Radelet and Siddiqi, 2007; Fan et al., 2013). First, a linear ordinary leastsquares (OLS) model assesses predictors of the logarithm of total per capita funding requested by a country for each disease per Global Fund round. Second, an ordered logistic regression model investigates each proposal's TRP rating (ranging from 1 to 4 ) using the same right-hand-side variables as Regression 1. To combine the effects of requested funding volumes per round (dependent variable in Regression 1) with approval rates (dependent variable in Regression 2) and the frequency with which a country applied for funding. Regression 3 uses OLS and the same functional form as Regression 1 to assess predictors of total per capita Global Fund funding received by country and by disease during the rounds-based mechanism. Finally, Regression 4 employs an ordered logistic model to investigate the performance ratings of signed grants as a function of TRP ratings of the underlying proposals and other program and country characteristics. Standard post-regression tests are conducted for data outliers, homoscedasticity, normality of residuals, and multi-collinearity of predictors.

Four limitations in this assessment relate to the underlying data. First, for the early years of Global Fund operations we lack adequate coverage for data relating to the availability of interventions to combat the three diseases, such as anti-retroviral therapy, long-lasting insecticide-treated bed nets, or comprehensive TB screening of atrisk patients. As a result, it is not possible to test for the presence of interventions that could be expected to be inversely related to funding needs (e.g. high bed nets prevalence would suggest lower residual funding volumes) or those where the association might be expected to be positive (e.g. high anti-retroviral therapy coverage implies high funding needs to sustain this coverage, particularly during the early years of the Global Fund when prices for these drugs were very high). However, it has been widely reported that coverage of these interventions was extremely low around 2002 (Binswanger, 2000; HLIRP, 2011), so one would only expect this latter relationship to become significant in the later years of the rounds-based mechanism, particularly after the large Round 8 in 2008. This lack of data for intervention coverage has a more significant impact on Regression 4 since we are unable to test if grant ratings are positively correlated with changes in intervention coverage.

A second data limitation concerns the quality of grant performance ratings, which has been questioned in the literature (Lu et al., 2006; Radelet and Siddiqi, 2007; Katz et al., 2010; Fan et al., 2013). We are unable to overcome these limitations, so Regression 4 can only establish the plausibility of consistent TRP assessment standards during the rounds-based mechanism.

Third, even though the R-squared for all 12 regressions are relatively high, the reported associations could be due to factors not considered in the models that were not related to intervention coverage.

Fourth, Global Fund practices, such as TRP procedures and evaluation standards, the role of the Technical Partners in supporting proposal design and implementation, and modalities for Principal Recipients and Local Fund Agents, improved over time. These effects may not have been picked up fully in the regression analyses. 
In addition to these data limitations, OLS and ordered logistic regression models have well-known weaknesses (Chatterjee and Hadi, 2015). They should therefore be complemented by case studies and other empirical techniques, which were beyond the scope of this chapter. Likewise, Chapter 6 does not assess the impact of shifting to the New Funding Model. We will consider the resulting suggestions for further research in the next section.

\subsection{Suggestions for further research}

\subsubsection{SDG metrics and country baselines}

The SDG Index and Dashboards introduced in Chapter 2 establish a preliminary and incomplete baseline for the SDGs. Further research may focus on closing gaps in available data, particularly with regards to international spill-over effects and sustainable consumption and production. Work undertaken by the author and collaborators subsequent to the paper on which Chapter 2 is based, introduces additional data into a revised and improved SDG Index, but some important data gaps remain (Sachs et al., 2017).

Another promising avenue for future research is to consider time-series data to assess whether countries are progressing sufficiently fast to achieve the SDGs by 2030 . Given the paucity of data and the infrequent collection of survey-based and other metrics, this would require extensive modeling of data to impute missing data and project trends. The Global Burden for Disease collaboration has conducted such analyses for the health SDGs (GBD, 2016) using complex modeling techniques. Yet it appears unlikely that these approaches can be applied to other SDG priorities where data availability and quality are far more limited than under the health goal. Another approach might be to focus on a smaller number of 'headline indicators' that can be modeled to generate trend data. For example, World Data Lab (2017) have published time-series estimates for extreme headcount poverty that correlate with other measures of extreme deprivation. A smaller set of similar 'headline indicators' may provide an initial assessment of countries' rate of progress towards the SDGs

In this thesis, we have illustrated in a preliminary way the usefulness of the SDG Index in understanding the determinants of subjective well-being. This opens promising research opportunities to deepen the analysis using cross-country regression techniques and to apply the method to other dependent variables measuring objectives of public policy.

To broaden the usefulness of the SDG Index and Dashboards for public policy, one may develop sub-national indices. As one example, Prakash et al. (2017b) propose a city-level SDG Index for the United States. Moreover, building on the concept of the augmented SDG Index for OECD countries introduced in Chapter 2, one may differentiate the SDG Index and Dashboards further by region, income groups or other country classifications to combine a common global core of SDG indicators with metrics that are particularly adapted to a set of countries in question. Such analyses promise to generate additional insights into the determinants of countries' SDG performance, particularly among countries at a similar stage of development or facing a common set of development challenges. 


\subsubsection{Public and private financing needs for the SDGs}

To deepen our understanding of investment needs for climate-resilient development (Chapter 3) and the SDGs more broadly (Chapter 4), four inter-related analytical issues need to be addressed. First, sector needs assessments related to the SDGs must be strengthened and updated, particularly for agriculture and food security, infrastructure, ecosystem services, and social protection. Using the analytical framework developed in this chapter, sector assessments can analyze changes in the composition and volume of total SDG investments needs (as opposed to considering only incremental investments), integrate investment needs related to climate change, remove overlaps and fill gaps between SDG investment areas, and consider how synergies and tradeoffs may be addressed. Needs assessments should also disaggregate between investments in tradables and non-tradables so that their results can be expressed in local currencies or international purchasing power parity. They must also pay greater attention to understanding how quickly private and public investments can be scaled up to achieve the SDGs by 2030. Integrated assessment models offer a promising method for generating consistent sector estimates that take account of interactions across sectors.

Second, to better guide national policymaking and to investigate the macroeconomic implications of achieving the SDGs, country SDG needs assessments and general equilibrium models for the SDGs should be developed. Such national assessments should consider synergies across SDG investment areas, investments in business capital, and economy-wide effects. Integrated assessment tools and expanded computable-general equilibrium models (such as the World Bank's MAMS) that span the full range of SDG investments are needed to support country-level assessments of investment needs. They should also integrate economic growth modeling and sector modeling to understand how countries can meet sector targets as well as macroeconomic objectives consistent with the SDGs (e.g. relating to income or employment). Such integrated analyses will also help compare results of SDG needs assessments with estimates of inclusive wealth (Arrow et al., 2012, 2013) or genuine savings (Hamilton and Clemens, 1999) required to achieve the SDGs.

Third, the timeframe for the analysis of investment needs for climate change adaptation needs to be extended through to the end of the SDG period in 2030 and likely beyond to 2050 , since the impacts of rising greenhouse gas emissions take a long time to feed through into development outcomes. Once extended to such a longer timeframe, analyses could differentiate between the different Representative Concentration Pathways (RCP) employed by the Fifth Assessment Report of the IPCC (IPCC, 2014).

Fourth, the indicative SDG financing framework identified in Chapter 4 needs to be improved by assessing total (as opposed to incremental) investment needs for the SDGs; developing a framework for domestic resource mobilization, including through government bonds under reasonable assumptions of debt sustainability; and determining the potential for non-concessional international public finance and the role of the multi-lateral development banks. The financing framework must be integrated into country-level growth models to obtain more robust estimates of the potential for longterm domestic resource mobilization and to design supportive macroeconomic policies. 


\subsubsection{Institutional mechanisms for delivering international development assistance}

The analysis of Global Fund design principles in Chapter 5 and recommendations made by the TRP (Chapter 6) generate several new research opportunities on appropriate institutional mechanisms for delivering international development assistance. In particular, a detailed comparison of the business models of other multilateral financing mechanisms with the design principles of the Global Fund would generate insights into how the effectiveness of these mechanisms can be improved and how lessons from the Global Fund can be applied in other areas. To our knowledge such an assessment has not been undertaken across SDG investment priorities, though the comparative advantages of health financing mechanisms have been investigated (Shakow, 2006; HLIRP, 2011). Such a comparison appears to be particularly relevant for the education sector, which has been underfunded under the MDGs (Schmidt-Traub and Sachs, 2015) and failed to accelerate progress in improving outcomes (McArthur and Rasmussen, 2017). UNESCO (2013) considers lessons from the Global Fund, but the analysis lacks the depth and rigor provided in Chapters 5 and 6.

With regards to the business model of the Global Fund, additional research is needed to analyze how the Global Fund has uncovered and then addressed weaknesses in its operating model. A richer evidence base in support of these questions, likely drawing on country case studies, might help further improve the design of the Global Fund and other mechanisms.

The panel data regressions developed in Chapter 6 could be complemented by case studies of how countries' grant proposals have evolved in light of the work of the TRP; medical best practice, including WHO treatment recommendations for HIVIAIDS, malaria, and other diseases (WHO, 2015b, 2016c); advice from Technical Partners; and other parameters. Likewise, such case studies could consider how the quality of proposals to the Global Fund has evolved using quantitative and qualitative parameters for each disease category and country.

Moreover, researchers may consider how the shift to the New Funding Model affected the scaling-up of overall funding volumes; country allocation of funding volumes, including financial suppression of grants from countries with large populations; and innovation in service proposal design and grant implementation. Rigorous assessments of these questions will help inform academic and policy debates on the appropriateness of different funding mechanisms for the SDGs.

Another research priority underscored by the findings in Chapter 6 concerns the performance of Global Fund grants. Following criticism by researchers and the TRP (TRP, 2009; Fan et al., 2013; Soeters, 2013), the organization has invested considerable effort in improving the system of performance ratings, so it would be interesting to investigate determinants of grant ratings, particularly for recent years of Global Fund operations.

Finally, more work is needed to determine whether and how lessons from the TRP can be applied to other multilateral financing mechanisms, such as the Global Environment Facility, the Green Climate Fund, the Global Partnership for Education, or the International Fund for Agricultural Development. 


\subsection{Policy implications}

\subsubsection{SDG metrics and country baselines}

Chapter 2 introduces the first analytical tool for tracking country-level progress towards the SDGs in a harmonized and comparable manner. It fills a major gap in available monitoring tools that either report regional aggregates with limited utility for countrylevel policy discussions (Nicolai et al., 2015; United Nations, 2017); focus on subsets of the SDGs (GBD, 2016); or rely on voluntary national reporting that lack comprehensiveness and international comparability (Bizikova and Pinter, 2017). By ranking country performance and by displaying complex data in easy-to-use dashboards, the SDG Index and Dashboards can help raise the prominence of the legally non-binding SDGs, which is a critical factor of success for international goals, as suggested by the MDG experience (McArthur, 2013; McArthur and Rasmussen, 2017). The examples of country rankings of education outcomes (Waldow, 2009; Wiseman, 2013), of human development (Dervis and Klugman, 2011), or of economic competitiveness (Besley, 2015) demonstrate that such rankings can trigger greater policy attention and action on complex development priorities. Another important feature of the SDG Index and Dashboards is that they are scale-invariant and can be applied at regional, national, and sub-national levels to support diagnoses of policy gaps and challenges at all levels of SDG implementation.

Our results show that the SDGs are a 'stretch agenda' for rich and poor countries alike. Every country scores 'red' on at least one SDG and many face challenges across several dimensions of sustainable development. As one example, the United States has the ninth highest per capita GDP in the world, but it ranks only $25^{\text {th }}$ on the SDG Index, notably due to relatively poor performance on environmental goals, inequality, and security. The development model of other high-income countries, such as members of the Gulf Cooperation Council, is similarly unbalanced.

Findings in Chapter 2 suggest that the 'universal agenda' of the SDGs (United Nations, 2015a) can be monitored for at least 149 countries using a common core of indicators. Data availability and development priorities differ substantially across countries, so it will be important to complement the global SDG Indicators with regionally appropriate metrics that reflect local needs and data availability. The augmented SDG Index for OECD countries reported in Chapter 2 illustrates how the global indicators can be combined with regional metrics. In this way, the SDG Index and Dashboards can provide a more granular analysis to complement the official, country-led voluntary reporting under the High-level Political Forum (Bizikova and Pinter, 2017).

We find that major data gaps exist across most SDG dimensions (Table 5, page 41), which in turn call for significant increases in funding for national and global data systems (Espey et al., 2015). But our analysis suggests that further changes are needed beyond increasing resources: Chapter 2 uses many non-official metrics from the scientific literature and other reputable sources to complement the data collected by national statistical offices and international organizations. Additional information on progress towards the SDGs may be obtained through the use of novel data types ('Big Data'), e.g. from social media or mobile phone networks. Yet so far, the SDG indicator process under the UN Statistical Commission has not incorporated indicators drawing on non-official metrics (Flückiger and Seth, 2016; UN Statistics Division, 2017), possibly because it is run by representatives from national statistical offices who tend to focus on traditional data sources. The official SDG indicator process is unlikely to resolve these methodological and data challenges rapidly since the next review is only scheduled for 2020. Therefore, scientists can help by making proposals for how the 
data gaps identified in this thesis can be closed and by launching initiatives to collect the necessary data.

As discussed in Chapter 2, data gaps are particularly prominent among measures for environmental goals, including sustainable consumption and production, where rich countries are expected to perform relatively poorly. For this reason, the current SDG Index likely overstates the SDG performance of richer countries. The 2017 SDG Index (Sachs et al., 2017) released subsequently to the publication on which Chapter 2 is based (Schmidt-Traub et al., 2017), addresses these gaps in part, but greater efforts are needed in closing remaining data gaps.

A focus ought to be placed on reducing the number of countries for which essential SDG indicators are unavailable, which tend to be extremely poor, emerging from conflict, and/or have small populations (Table 12, page 65). In particular, socioeconomic data based on surveys and environmental metrics requiring sophisticated monitoring systems tend to be sparse in these countries. It is encouraging that the second edition of the SDG Index and Dashboards (Sachs et al., 2017) includes an additional 8 countries, but greater efforts are needed to close data gaps for core SDG variables, including through increasing resources for data collection, developing improved survey tools for small populations, and greater use of remote sensing technologies. International organizations may prioritize filling these data gaps in international SDG data sets, including through imputation and estimation techniques, where possible and appropriate.

\subsubsection{Public and private financing needs for the SDGs}

Policy conclusions from Chapters 3 and 4 are tentative given the preliminary and global nature of the analyses as well as their reliance on some studies that have not undergone rigorous peer review. With these caveats in mind, we identify six policy implications from our investigations into financing needs for climate-resilient development in Africa and the SDGs.

First, meeting the SDGs and climate-proofing development strategies will require significant additional resources, but these appear manageable given the size of world GDP and global savings. Countries in sub-Saharan Africa will likely face an additional $40 \%$ in annual incremental development financing needs to address climate change adaptation. To achieve the SDGs, the world will need to mobilize an additional $\$ 2.3$ trillion per year in public and private investments. Accounting for $1.5-2.5 \%$ of average annual world GDP through to 2030 or some 10 percent of global savings, this volume of incremental financing is substantial but appears manageable, particularly since it will partially offset GDP losses under a business-as-usual scenario (e.g. Stern (2007) and IPCC (2014)). Therefore, the world does not face a financing constraint per se in achieving the SDGs. The question instead becomes how the appropriate combination of public and private financing can be mobilized and invested, including through international public and private finance.

Second, it is critical to complement assessments of sectoral investment needs with analyses of total financing needs, but this is currently not done routinely in the design of intergovernmental agreements or the supporting policy analysis. Simply adding up sector investment needs without addressing overlaps and synergies, as done for example by UNTT (2013) and reproduced by ICESDF (2014), yields investment needs that are vastly higher than suggested by the analyses proposed in this thesis. This 
point is further supported by integrated partial equilibrium analyses across several sectors, which consistently suggest significant synergies across sector investment needs (e.g. McCollum et al. $(2013,2012)$ ).

On the other hand, sector-based financing analyses that do not consider overall resource needs for the SDGs tend to overstate poor countries' ability to mobilize domestic public financing. For example, Hagen-Zanker and McCord (2011) show that domestic resource mobilization targets across the major SDG sectors, including education, health, agriculture, and infrastructure, exceed domestic resource mobilization potential under any reasonable macroeconomic assumptions. Our SDG-based investment analysis in Chapter 4 suggests meanwhile that all low- and some lower-middle-income countries will require greater concessional public financing to achieve the SDGs. The IMF and other international organizations supporting the SDGs should therefore develop integrated macroeconomic assessments for the SDGs as pioneered under the MDGs by IMF (2008) and Mongardini and Samake (2009).

The consideration of sector and aggregate investment needs must start in intergovernmental agreements that set the framework for international cooperation on sustainable development. The three major agreements struck in 2015, Agenda 2030 (United Nations, 2015a), the Paris Agreement (UNFCCC, 2015), and Financing for Development (United Nations, 2015b), do not provide integrated resource estimates for sustainable development, and they do not call for the generation of such estimates. This omission is a significant analytical and policy gap in the world's efforts to achieve the SDGs and implement the Paris Climate Agreement (see also SDSN (2015b)). The gap could be filled by upcoming COPs, the High-Level Political Forum overseeing the implementation of the SDGs, and/or the follow-up process under Addis Ababa Action Agenda that could each agree to conduct integrated investment needs assessments for the SDGs at country and regional levels.

Third, governments need to consider, within each investment area, how climate change adaptation measures and development interventions can be integrated to ensure effective implementation. The analysis in Chapter 3 suggests that it is important to implement adaptation measures under the oversight of the same ministries that are responsible for delivering related development outcomes. Chapter 4 proposes how the 17 SDGs can be organized into eight SDG investment areas that may help guide the organization and design of governments' implementation strategies across responsible line ministries. Together the chapters show how sector-based assessments can be aligned with the organizational structure of governments so that financing needs and results can be tied to explicit objectives and delivery mechanisms.

A related issue not analyzed in this thesis is the intertemporal structuring and sequencing of investments to achieve the SDGs and implement the Paris Climate Agreement. The Deep Decarbonization Pathways Project (Bataille et al., 2016; Sachs et al., 2016c) has shown inter alia that achieving the structural transformations needed to decarbonize energy systems will require mid-century development strategies. Pursuing only short-term strategies, such as the Nationally-Determined Contributions under the Paris Climate Agreement, may lead to lock-in of unsustainable technologies and will increase the costs of the transformations even if the short-term strategies are ambitious (Sachs et al., 2016c). Similar challenges of intertemporal sequencing and the need for a long-term investment perspective likely arise in other areas, such as land use and the management of marine resources (Schmidt-Traub, 2017). In response to the findings of the DDPP, the Paris Climate Agreement has included Article 4.19, which calls on all countries to develop and submit by 2020 "low-emission development strategies" consistent with the Convention's Objective to keep the rise in average global temperatures to "well below $2^{\circ} \mathrm{C}$ " (UNFCCC, 2015). The needs assessment methodologies 
and findings proposed in Chapters 3 and 4 may help define and organize the long-term pathways required to operationalize Article 4.19.

A fourth and closely related policy conclusion is that the findings from Chapters 3 and 4 may help refine the definition of 'additional' climate finance. In the absence of an agreed baseline for 'development finance' it becomes difficult to determine how the additionality of climate finance be assessed and how achievement of the commitment by developed countries to provide $\$ 100$ billion in additional climate finance be monitored. As a result, the definition of additionality has become a contentious issue in the climate negotiations with many developed countries refusing to adopt a consistent and time-invariant definition (Buchner et al., 2014; SDSN, 2015b; OECD and CPI, 2015).

The methodology developed in Chapter 3 allows for the integration of development and climate adaptation investments, but it also permits the analytical separation of development and climate adaptation funding needs to determine 'additionality' of climate finance consistent with UNFCCC rules. Under such an approach, resources for adaptation could be deemed additional if they are provided above and beyond the nonclimate financing needs for development, as recommended by SDSN (2015b). This approach to defining additionality has the benefit of being applicable across all developed countries. It is consistent with the intention of the Paris Climate Agreement, but sets a higher bar for additionality than the approach proposed by OECD and CPI (2015).

Fifth, the private sector can make significant contributions to financing the SDGs, but likely not more than $45 \%$ of total investment needs (Chapter 4 ). As also underscored by UNCTAD (2014), opportunities for private financing vary significantly across sectors and increase with rising per capita incomes. The analysis supports the larger focus on private financing in the Addis Ababa Action Agenda (United Nations, 2015b) relative to the 2001 Monterrey Consensus (United Nations, 2002), but it also reaffirms the need for greater public investments suggesting limited substitutability between the two.

The importance of private sector financing for the SDGs reinforces the need to improve the quality and coverage of data on international development finance flows beyond ODA, as measured by the OECD Development Assistance Committee. For example, estimates of foreign direct investments provided by the OECD, IMF, and UNCTAD use different definitions and data. As a result, they are difficult to compare. We also lack agreed definitions and robust data on private finance leveraged through concessional or non-concessional public financing (SDSN, 2015b), though OECD and CPI (2015) propose a first methodology for measuring private climate finance leveraged through public climate finance. More comprehensive estimates of private investment flows by international organizations, such as the IMF, OECD, or UNCTAD, will help generate clarity on shortfalls in financing for the SDGs and in implementing the Paris Climate Agreement. In view of the complexity and political sensitivities surrounding these measurement issues, the methodologies and data should be transparent and subject to rigorous external review (see Schmidt-Traub and Sachs (2015) for a detailed discussion of these measurement issues).

Sixth, our findings show that ODA will need to increase significantly if the SDGs are to be achieved in low-income countries. Lower-middle-income countries present an intermediate case. As a group, they may not require significant volumes of ODA to achieve the SDGs, but it appears likely that some LMICs will require ODA, particularly during the early years of SDG implementation when GDP per capita is still relatively low compared with aggregate investment needs. Kharas (2014) and Kharas et al. (2014) also find that LMICs may require significant incremental ODA. 


\subsubsection{Institutional mechanisms for delivering international development assistance}

The findings in Chapters 5 and 6 suggest that the Global Fund has successfully scaled up grant resources for complex control and treatment programs targeting HIVIAIDS, $\mathrm{TB}$, and malaria. It is notable that the Global Fund has performed well across the spectrum of operating environments, including fragile countries that have recently emerged from conflict. Moreover, the Global Fund has had significant impact on implementation research and improving national practices for disease management as well as global treatment guidelines (WHO, 2015b, 2016c). The Global Fund's unique design principles (GFATM, 2001; Sachs and Schmidt-Traub, 2017) have helped the world move rapidly from a situation where developing countries were not designing and implementing national-scale strategies to control and treat HIVIAIDS, TB, and malaria (Binswanger, 2000; Stop TB Partnership, 2000; Yamey, 2004; HLIRP, 2011) to one where this knowledge has become widespread and is applied in most developing countries, including fragile and poorly governed countries (Roll Back Malaria Partnership, 2008; Jamison et al., 2013; UNAIDS, 2014; Stop TB Partnership, 2015). This experience suggests that rapid progress towards international development goals may be possible when adequate financing is made available through well-designed pooled funding mechanisms.

The Global Fund performance illustrates how the competing needs of country ownership and technical rigor of funding proposals, which we discussed in Chapter 1, can be reconciled using independent technical review processes along the lines of the TRP. The case of China is illustrative: For political and geostrategic reasons, most bilateral donors would have likely found it impossible to reject China's early HIVIAIDS proposals that did not include harm reduction programs, but the TRP did so during the first two rounds despite China's protestations (Minghui et al., 2015). The repeated rejections stimulated a debate in China on how best to tackle the infectious diseases and led to deep changes in the country's strategies and institutions, which brought them in line with international medical best practice and have since been credited with substantial improvements in health outcomes (Wang et al., 2014; Minghui et al., 2015). Similar experiences of 'learning' have unfolded in many other countries under Global Fund-supported programs.

The evidence from Chapter 6 suggests further that independent reviews based on the model of the TRP can generate funding allocations that are in line with countries' needs as measured by disease burden and per capita income. In particular, the Global Fund's strong track record in fragile countries shows that demand-led financing can be needsbased if it is underpinned by adequate technical support, as provided by the Global Fund's Technical Partners and other bilateral mechanisms. This finding may have important policy implications for other sectors that lack effective mechanisms for reconciling the trade-off between needs-based and demand-led funding allocations (Temple, 2010).

In summary, the Global Fund offers a model for managing the two tensions identified in Chapter 1: First, reconciling country ownership and the need to ensure effective use of scarce resources (e.g. consistent with medical best practice in the case of health) and, second, reconciling bottom-up demand-led programming of resources and the imperative to channel resources to the countries most in need that may have low capacity to formulate, implement, and monitor complex disease control programs. This makes the Global Fund well aligned with the aid effectiveness principles of the 2005 Paris Declaration and its successors (OECD, 2008). 
These findings carry important policy implications for the Global Fund and the financing of other SDG priorities. For the Global Fund, the findings reinforce the attractiveness of a demand-led competitive funding process. If the TRP can withhold funding altogether for programs that are not consistent with the latest medical evidence then this exerts a powerful incentive effect on countries' design and implementation of disease control strategies, as illustrated by the case of China discussed above. It is not clear if the Global Fund's New Funding Model, which sharply reduces the TRP's discretion in allocating funding volumes, is fully aligned with these principles.

The policy implications might be even more significant for bilateral and other multilateral funding mechanisms. Bilateral mechanisms tend to base funding allocations and decisions inter alia on geopolitical and bilateral political considerations. In essence, bilateral aid is to a large extent a tool of international diplomacy, as confirmed by a large number of studies into aid allocations (Alesina and Dollar, 2000; Shakow, 2006; Younas, 2008; Bourguignon et al., 2009; Temple, 2010; Dreher et al., 2015). Similarly, multilateral mechanisms reviewed by DFID (2016), with the exception of Gavi, do not perform independent evaluations of grant proposals that determine funding decisions. The Global Fund model investigated in Chapters 5 and 6 suggests that the TRP and the seven other key design principles of the Global Fund may hold useful lessons for these mechanisms.

The two chapters therefore lend support to recommendations made in the policy literature for the multilateral pooling of official development assistance (SDSN, 2015b; Schmidt-Traub and Sachs, 2015). Working closely with Gavi, the Global Fund should serve as the primary funding vehicle to implement strategies to break the three epidemics (90-90-90 Strategy for HIV/ AIDS, the Global Plan to End TB, Global Malaria Action Plan) in order to achieve SDG Target 3.3 and to finance universal health coverage (SDG 3.8). This would require a broadening of the Global Fund's business model to expand investments in health systems as well as significant additional resources beyond the $\$ 13$ billion raised during the 2017-2019 replenishment round hosted by the Government of Canada.

The Global Fund model may also help meet investment needs in non-health SDG areas where proven interventions need to be scaled up with the help of public (co-)financing. This applies in particular to the education sector (UNESCO, 2013, 2015b). The recently announced Global Emergency Education Fund could be a step in the right direction for the education goals (SDG 4). Together with the underfunded Global Partnership for Education, they should apply the design principles of the Global Fund, possibly rebranding themselves as an integrated Global Fund for Education.

Other SDG priorities that may consider the Global Fund model include smallholder farming and improved nutrition (SDGs 2 and 3) where the International Fund for Agricultural Development (IFAD) and the Global Agriculture and Food Security Program (GAFSP) hosted by the World Bank act as the principal multilateral funders; access to safe water and sanitation (SDG 6), possibly drawing on the existing Global Sanitation Fund; distributed rural electrification (SDG 7) building on Sustainable Energy for All (S4All) or mechanisms at the regional development banks and the World Bank. In the area of climate change (SDG 13), the Green Climate Fund has come under significant public criticism for its operations and may benefit particularly from applying Global Fund lessons. Finally, the Global Environment Facility (GEF) is the leading multilateral funding mechanisms for biodiversity and ecosystem services, but it operates at a volume that is far too low given funding needs for the SDGs (Schmidt-Traub, 2015). As the GEF gears up for its next replenishment cycle (GEF-7) considering lessons from the Global Fund may be particularly timely. 
The Global Fund model appears less suited for SDG investment areas that require more market- and project-based financing solutions. This includes infrastructure financing, which tends to involve large numbers of equity and debt providers around project-based financing structures. Here multilateral development banks, such as the World Bank, the Asian Infrastructure Investment Bank, or the regional development banks, may play a more important role in pooling resources to reduce unnecessary transaction costs.

In conclusion, the analyses in this thesis lend support to the notion that the SDGs can serve as useful tools for international cooperation around sustainable development. It is possible to establish reasonably comprehensive SDG baselines for the vast majority of UN member states, though important data gaps remain, and the international system led by the UN Statistics Commission has been slow to consider the full range of data sources available for the SDGs. The SDGs also provide a useful framework for assessing public and private investment needs for sustainable development overall and by sector. Though still relatively poorly understood, these investment needs appear manageable relative to world GDP and global savings. It appears critical that the scientific and policy literature improve our understanding of SDG investment needs and how they can be met. Since money alone cannot achieve global goals and many development programs fail to meet their objectives, it is important to better understand how relatively successful financing mechanisms, such as the Global Fund, operate. This thesis suggests that the unique design principles of the Global Fund, particularly the independent review of country-led proposals, provide a model for scaling up international public financing for other SDG investment areas. However, the design of the institutional mechanisms will need to be tailored to the specific needs in each sector. 
On metrics and financing for the Sustainable Development Goals 
References 
Acharya, A., A.T.F. De Lima, and M. Moore (2006), "Proliferation and fragmentation: Transactions costs and the value of aid," The Journal of Development Studies, 42(1), 1-21.

Adam, C., G. Chambas, P. Guillaumont, S.G. Jeanneney, and J.W. Gunning (2004), "Performance-based conditionality: A European perspective," World Development, 32(6), 1059-1070.

Adams, G., M. Addo, A. Aldovini, M. Altfeld, and D. Anderson (2001), "Consensus statement on antiretroviral treatment for AIDS in poor countries," Topics in HIV Medicine, 9(2), 14-26.

Agénor, P.-R., N. Bayraktar, E. Pinto Moreira, and K. El Aynaoui (2005), "Achieving the Millennium Development Goals in Sub-Saharan Africa: A Macroeconomic Monitoring Framework," Policy Research Working Paper No. 3750, World Bank, Washington D.C.

AGF (2010), "Report of the Secretary General's High Level Advisory Group on Climate Change Financing," Advisory Group on Climate Change, New York.

Agrawala, S., and S. Fankhauser (2008), Putting climate change adaptation in an economic context, OECD Publishing, Paris.

Aidspan (2017), Guides to the Global Fund, http://www.aidspan.org/page/guidesglobal-fund, accessed July 10, 2017.

Alesina, A., and D. Dollar (2000), "Who gives foreign aid to whom and why?," Journal of Economic Growth, 5(1), 33-63.

Allen, C., G. Metternicht, and T. Wiedmann (2016), "National pathways to the Sustainable Development Goals (SDGs): A comparative review of scenario modelling tools," Environmental Science \& Policy, 66, 199-207.

AMCEN (2008), "Climate Change Adaptation in Africa," Scoping Study for the Expert Group Meeting, African Ministerial Conference on Africa, Addis Ababa.

Annan, K., N. Desai, J. Egeland, S. Huq, A. Merkl, R. Pachauri, J. Rockström, J.D. Sachs, H. Schellnhuber, B. Stocking, K. Töpfer, and M. Wahlström (2009), "Human impact report: Climate change. The anatomy of a silent crisis," Global Humanitarian Forum, Geneva.

Arrow, K.J., H.B. Chenery, B.S. Minhas, and R.M. Solow (1961), "Capital-Labor Substitution and Economic Efficiency," The Review of Economics and Statistics, 43(3), 225-250.

Arrow, K.J., P. Dasgupta, L.H. Goulder, K.J. Mumford, and K. Oleson (2012), "Sustainability and the measurement of wealth," Environment and Development Economics, 17(03), 317-353.

Arrow, K.J., P. Dasgupta, L.H. Goulder, K.J. Mumford, and K. Oleson (2013), "Sustainability and the measurement of wealth: further reflections," Environment and Development Economics, 18(4), 504-516.

Atteridge, A., B. Müller, A. Persson, T. Takama, J. Hoffmaister, M. Lazarus, R.J. Klein, and C. Kehler Siebert (2009), "Adaptation finance under a Copenhagen agreed outcome," Stockholm Environment Institute, Stockholm.

Atun, R., and M. Kazatchkine (2009), "Promoting country ownership and stewardship of health programs: The Global Fund experience," Journal of Acquired Immune Deficiency Syndromes (1999), 52 Suppl 1, S67-68.

(2010), "The Global Fund's leadership on harm reduction: 2002-2009," International Journal of Drug Policy, 21(2), 103-106.

Atun, R., S.K. Pothapregada, J. Kwansah, D. Degbotse, and J.V. Lazarus (2011), "Critical interactions between the Global Fund-supported HIV programs and the health system in Ghana," Journal of Acquired Immune Deficiency Syndromes (1999), 57 Suppl 2, S72-76.

Bahadur, C., M. Kruk, and G. Schmidt-Traub (2006), "Preparing National Strategies to Achieve the MDGs: A Handbook," UN Millennium Project, New York. 
Banerjee, A.V. (2008), "Big answers for big questions: the presumption of growth policy," in: Brookings Conference What Works in Development, Brookings Institution, Washington D.C.

Barder, O.M., and N. Birdsall (2006), "Payments for progress: a hands-off approach to foreign aid," CGD Working Paper No. 102, Center for Global Development, Washington D.C.

Barr, R., S. Fankhauser, and K. Hamilton (2010), "Adaptation investments: a resource allocation framework," Mitigation and Adaptation Strategies for Global Change, 15(8), 843-858.

Bass, E. (2005), "Uganda is learning from its Global Fund grant suspension," The Lancet, 366(9500), 1839-1840.

Bataille, C., H. Waisman, M. Colombier, L. Segafredo, J. Williams, and F. Jotzo (2016), "The need for national deep decarbonization pathways for effective climate policy," Climate Policy, 16(sup1), S7-S26.

Behrman, G. (2008), The Invisible People: How the US Has Slept Through the Global AIDS Pandemic, Simon and Schuster, New York.

Benson, C., and E.J. Clay (1998), The impact of drought on sub-Saharan African economies: a preliminary examination, World Bank.

Berg, E. (2001), "Why Are Aid Organizations Such Poor Learners," in: J. Carlsson, L. Wohlgemuth (eds.), Learning in Development Cooperation, Almqvist and Wiksell International, Stockholm.

Besley, T. (2015), "Law, regulation, and the business climate: The nature and influence of the World Bank Doing Business project," The Journal of Economic Perspectives, 29(3), 99-120.

Bhattacharya, A., M. Romani, and N. Stern (2012), Infrastructure for development: meeting the challenge, Centre for Climate Change Economics and Policy, London.

Binswanger, H.P. (2000), "Scaling up HIVIAIDS programs to national coverage," Science, 288(5474), 2173-2176.

BirdLife International, IUCN, and UNEP-WCMC (2017), "Marine Important Bird and Biodiversity Areas," BirdLife International, International Union for Conservation of Nature and United Nations Environment Programme - World Conservation Monitoring Center, Geneva.

Bizikova, L., and L. Pinter (2017), "Indicator Preferences in National Reporting of Progress Toward the Sustainable Development Goals," International Institute for Sustainable Development, Winnipeg.

Blackorby, C., and D. Donaldson (1982), "Ratio-Scale and Translation-Scale Full Interpersonal Comparability without Domain Restrictions: Admissible SocialEvaluation Functions," International Economic Review, 23(2), 249.

Boko, M., I. Niang, A. Nyong, C. Vogel, A. Githeko, M. Medany, B. Osman-Elasha, R. Tabo, and P. Yanda (2007), Africa. Climate Change 2007: Impacts, Adaptation and Vulnerability. Contribution of Working Group II to the Fourth Assessment Report of the Intergovernmental Panel on Climate Change, eds. ML Parry, OF Canziani, JP Palutikof, PJ van der Linden and CE Hanson, 433-467, Cambridge University Press, Cambridge, UK.

Boone, P. (1996), "Politics and the effectiveness of foreign aid," European Economic Review, 40(2), 289-329.

Booysen, F. (2002), "An Overview and Evaluation of Composite Indices of Development," Social Indicators Research, 59(2), 115-151.

Bornemisza, O., J. Bridge, M. Olszak-Olszewski, G. Sakvarelidze, and J.V. Lazarus (2010), "Health Aid Governance in Fragile States: The Global Fund Experience," Global Health Governance, 4(1), 1-18.

Bourguignon, F., C. Diaz-Bonilla, and H. Lofgren (2008), "Aid, Service Delivery, and the Millennium Development Goals in an Economy-wide Framework," Policy 
Research Paper No. 4683, The World Bank Development Economics Prospects Group, World Bank, Washington, D.C.

Bourguignon, F., V. Levin, and D. Rosenblatt (2009), "International redistribution of income," World Development, 37(1), 1-10.

Bowser, D., S.P. Sparkes, A. Mitchell, T.J. Bossert, T. Bärnighausen, G. Gedik, and R. Atun (2014), "Global Fund investments in human resources for health: innovation and missed opportunities for health systems strengthening," Health Policy and Planning, 29(8), 986-997.

Bridge, J., B.M. Hunter, E. Albers, C. Cook, M. Guarinieri, J.V. Lazarus, J. MacAllister, S. McLean, and D. Wolfe (2016), "The Global Fund to Fight AIDS, Tuberculosis and Malaria's investments in harm reduction through the rounds-based funding model (2002-2014)," International Journal of Drug Policy, 27, 132-137.

Broadband Commission (2014), "Means of Transformation harnessing broadband for the post-2015 development agenda," United Nations Educational Scientific and Cultural Organization, International Telecommunication Union, in collaboration with Ericsson, Geneva.

Brown, J.C., and W. Griekspoor (2013), "Fraud at the Global Fund? A viewpoint," The International Journal of Health Planning and Management, 28(1), 138-143.

Brundtland, G., M. Khalid, S. Agnelli, S. Al-Athel, B. Chidzero, L. Fadika, V. Hauff, I. Lang, M. Shijun, M.M. de Botero, and M. Singh (1987), Our Common Future, Oxford University Press, New York.

Buchner, B., D. Abramskiehn, M. Stadelmann, J. Wilkinson, A. Rosenberg, and F. Mazza (2014), "The global landscape of climate finance 2014," Climate Policy Initiative, San Francisco.

Burnside, C., and D. Dollar (2000), "Aid, policies, and growth," American Economic Review, 90(4), 847-868.

Car, J., T. Paljärvi, M. Car, A. Kazeem, A. Majeed, and R. Atun (2012), "Negative health system effects of Global Fund's investments in AIDS, tuberculosis and malaria from 2002 to 2009: systematic review," JRSM Short Reports, 3(10), 70.

Carter, M.R., P.D. Little, T. Mogues, and W. Negatu (2007), "Poverty traps and natural disasters in Ethiopia and Honduras," World Development, 35(5), 835-856.

CBD (2012a), "Full Assessment of the Amount of Funds Needed for the Implementation of the Convention for the Sixth Replenishment Period of the Trust Fund of the Global Environment Facility," Convention on Biological Diversity, Hyderabad.

(2012b), "Resourcing the Aichi Biodiversity Targets: A first assessment of the resources required for implementing the strategic plan for biodiversity 20112020.," Convention on Biological Diversity, Montreal.

Chandy, L., and H. Kharas (2011), "The Practical Limits to International Development Cooperation: Why Can't We All Just Get Along?," Journal of International Development, 23(5), 739-751.

Chatham House (2014), "Shared Responsibilities for Health A Coherent Global Framework for Health Financing," Final Report of the Centre on Global Health Security Working Group on Health Financing, Chatham House, London.

Chatterjee, S., and A.S. Hadi (2015), Regression analysis by example, 5th ed., John Wiley \& Sons, Hoboken, New Jersey.

Chipperfield, M.P., S.S. Dhomse, W. Feng, R.L. McKenzie, G.J.M. Velders, and J.A. Pyle (2015), "Quantifying the ozone and ultraviolet benefits already achieved by the Montreal Protocol," Nature Communications, 6, 7233.

Chomitz, K. (2007), "Overview at loggerheads? Agricultural Expansion, Poverty Reduction and Environment in the Tropical Forests," World Bank, Washington D.C.

CIA (2016), The CIA World Factbook, https://www.cia.gov/library/publications/theworld-factbook/, accessed December 7, 2016. 
Clark, A.E., and A.J. Oswald (1994), "Unhappiness and Unemployment," The Economic Journal, 104(424), 648-659.

Clemens, M.A., C.J. Kenny, and T.J. Moss (2007), "The Trouble with the MDGs: Confronting Expectations of Aid and Development Success," Working Paper No. 40, Center for Global Development, Washington D.C.

CMH (2001), "Macroeconomics and Health: Investing in Health for Economic Development," Report of the Commission on Macroeconomics and Health, World Health Organization, Geneva.

- (2003), Investing in health: A summary of the findings of the Commission on Macroeconomics and Health, Commission on Macroeconomics and Health, World Health Organization, Geneva.

Collier, P. (2008), The bottom billion: Why the poorest countries are failing and what can be done about it, Oxford University Press, New York.

Collier, P., G. Conway, and T. Venables (2008), "Climate change and Africa," Oxford Review of Economic Policy, 24(2), 337-353.

Collier, P., and D. Dollar (2002), "Aid allocation and poverty reduction," European Economic Review, 46(8), 1475-1500.

- (2004), "Development effectiveness: what have we learnt?," The Economic Journal, 114(496), 244-271.

Commission for Africa (2005), Our Common Interest: An Argument, Penguin UK, London, UK.

Dasgupta, P., and K.-G. Mäler (2000), "Net national product, wealth, and social wellbeing," Environment and Development Economics, 5(01), 69-93.

de Jongh, T.E., J.H. Harnmeijer, R. Atun, E.L. Korenromp, J. Zhao, J. Puvimanasinghe, and R. Baltussen (2014), "Health impact of external funding for HIV, tuberculosis and malaria: systematic review," Health Policy and Planning, 29(5), 650-662.

Delamonica, E., S. Mehrotra, and J. Vandemoortele (2001), "Is EFA affordable? Estimating the minimum cost of 'Education for All,'” Innocenti Working Paper No. 87, United Nations Children's Fund, Florence.

Delhey, J., and C. Kroll (2013), "A 'Happiness Test' for the New Measures of National Well-Being: How Much Better than GDP are They?," in: H. Brockmann, J. Delhey (eds.), Human Happiness and the Pursuit of Maximization: Is More Always Better?, Springer Netherlands, Dordrecht, pp. 191-210.

Dell, M., B.F. Jones, and B.A. Olken (2009), "Temperature and income: reconciling new cross-sectional and panel estimates," American Economic Review Papers and Proceedings, 99(2), 198-204.

Dervis, K., and J. Klugman (2011), "Measuring human progress: the contribution of the Human Development Index and related indices," Revue d'Economie Politique, 121(1), 73-92.

Devarajan, S., M.J. Miller, and E.V. Swanson (2002), "Goals for Development: History, Prospects and Costs," World Bank, Washington, D.C.

Development Initiatives (2015), "Getting poverty to zero: financing for social protection in least developed countries," Development Initiatives, Bristol.

DFID (2016), "Raising the Standard: the Multilateral Development Review 2016," Department for International Development, London.

Dizon-Ross, R., P. Dupas, and J. Robinson (2015), "Governance and the Effectiveness of Public Health Subsidies," No. NBER Working Paper No. 21324, National Bureau of Economic Research.

Dolan, P., T. Peasgood, and M. White (2008), "Do we really know what makes us happy? A review of the economic literature on the factors associated with subjective well-being," Journal of Economic Psychology, 29(1), 94-122.

Donner, S.D., M. Kandlikar, and S. Webber (2016), "Measuring and tracking the flow of climate change adaptation aid to the developing world," Environmental Research Letters, 11(5), 054006. 
Dreher, A., P. Nunnenkamp, and M. Schmaljohann (2015), "The Allocation of German Aid: Self-interest and Government Ideology," Economics \& Politics, 27(1), 160184.

Dreher, A., P. Nunnenkamp, and R. Thiele (2008), "Does aid for education educate children? Evidence from panel data," The World Bank Economic Review, 22(2), 291-314.

Easterly, W. (2005), "How to assess the need for aid? The answer: don't ask," Working Paper No.18, NYU Development Research Institute. New York University, New York.

- (2006), The White Man's Burden, Penguin Press, New York.

Easterly, W. (2007), "Are aid agencies improving?," Economic Policy, 22(52), 634678.

ECONADAPT (2015), "The Costs and Benefits of Adaptation. Results from the ECONADAPT Project," ECONADAPT Consortium, Brussels.

ECOSOC (2015), "Report of the Inter-Agency and Expert Group on Sustainable Development Goal Indicators," No. E/CN.3/2016/2, United Nations Economic and Social Council, New York.

Espey, J., E. Swanson, S. Badiee, Z. Christensen, A. Fischer, M. Levy, G. Yetman, A. de Sherbinin, R. Chen, Y. Qu, G. Greenwell, T. Klein, J. Jutting, M. Jerven, G. Cameron, A. Rivera, A.M. Arias, and C.V. Lantei Mills (2015), "Data for Development: A Needs Assessment for SDG Monitoring and Statistical Capacity Development," Sustainable Development Solutions Network, Paris and New York.

Fan, V.Y., D. Duran, R. Silverman, and A. Glassman (2013), "Performance-based financing at the Global Fund to Fight AIDS, Tuberculosis and Malaria: an analysis of grant ratings and funding, 2003-12," The Lancet Global Health, 1(3), e161-e168.

Fan, V.Y., A. Glassman, and R.L. Silverman (2014), "How a new funding model will shift allocations from the Global Fund to Fight AIDS, tuberculosis, and malaria," Health Affairs (Project Hope), 33(12), 2238-2246.

Fankhauser, S. (2010), "The Costs of Adaptation," Wiley Interdisciplinary Reviews: Climate Change, 1(1), 23-30.

Fankhauser, S., and I. Burton (2011), "Spending adaptation money wisely," Climate Policy, 11(3), 1037-1049.

Fankhauser, S., and G. Schmidt-Traub (2011), "From Adaptation to Climate-Resilient Development. The cost of climate-proofing the Millennium Development Goals in Africa," Climate and Development, 3(2).

FAO (2015), "Prevalence of undernourishment (\% of population)," Food and Agriculture Organization.

(2017a), Cereal yield (kg per hectare) | Data, http://data.worldbank.org/indicator/AG.YLD.CREL.KG, accessed January 4, 2017.

(2017b), AQUASTAT database, http://www.fao.org/nr/water/aquastat/data/query/index.html?lang=en, accessed January 4, 2017.

FAO, IFAD, and WFP (2015a), "The State of Food Insecurity in the World 2015. Meeting the 2015 international hunger targets: taking stock of uneven progress.," Food and Agriculture Organization, International Fund for Agricultural Development and World Food Programme.

(2015b), "Achieving Zero Hunger: The Critical Role of Investments in Social Protection and Agriculture," Food and Agriculture Organization, International Fund for Agricultural Development and World Food Programme, Rome.

Farmer, P., F. Léandre, J.S. Mukherjee, M. Claude, P. Nevil, M.C. Smith-Fawzi, S.P. Koenig, A. Castro, M.C. Becerra, J.D. Sachs, A. Attaran, and J.Y. Kim (2001), "Community-based approaches to HIV treatment in resource-poor settings," The Lancet, 358(9279), 404-409.

Fassin, D., and H. Schneider (2003), "The politics of AIDS in South Africa: beyond the controversies," BMJ: British Medical Journal, 326(7387), 495. 
Fay, M., M. Toman, D. Benitez, and S. Csordas (2011), "Infrastructure and sustainable development," in: S. Fardoust, Y. Kim, C. Sepulveda (eds.), Postcrisis Growth and Development: A Development Agenda for the G20, World Bank, Washington, D.C., pp. 329-382.

Ferreira, F., S. Chen, A. Dabalen, Y. Dikhanov, N. Hamadeh, and D. Jolliffe (2015), "A global count of the extreme poor in 2012: data issues, methodology and initial results," World Bank, Washington D.C.

Flückiger, Y., and N. Seth (2016), "Sustainable Development Goals: SDG indicators need crowdsourcing," Nature, 531(7595), 448-448.

Forouzanfar, M.H., A. Afshin, L.T. Alexander, H.R. Anderson, Z.A. Bhutta, S. Biryukov, M. Brauer, R. Burnett, K. Cercy, F.J. Charlson, and others (2016), "Global, regional, and national comparative risk assessment of 79 behavioural, environmental and occupational, and metabolic risks or clusters of risks, 1990-2015: a systematic analysis for the Global Burden of Disease Study 2015," The Lancet, 388(10053), 1659.

Foster, V., and C. Briceño-Garmendia (2010), "Africa's Infrastructure: A Time for Transformation," World Bank, Washington, D.C.

French Ministry of Foreign Affairs (2013), "French Contributions to the Global Fund to Fight AIDS, Tuberculosis and Malaria: Evaluation Report Synthesis," No. 126, French Ministry of Foreign Affairs, Paris.

Fund for Peace (2016), "Fragile States Index 2016," Fund for Peace, Washington D.C.

Gable, S., H. Lofgren, and I. Osorio-Rodarte (2015), "Country Development Diagnostics Post-2015," World Bank, Washington, D.C.

Gallup (2016), "Gallup World Poll," Gallup, New York.

Garau, P., E. Sclar, and G.Y. Carolini (2005), A Home in the City, Earthscan, London.

Gavi (2014), "The 2016-2020 Investment Opportunity," Global Alliance for Vaccines and Immunizations, Geneva.

GBD (2016), "Measuring the health-related Sustainable Development Goals in 188 countries: a baseline analysis from the Global Burden of Disease Study 2015," The Lancet, 388(10053), 1813-1850.

GEA (2012), Global Energy Assessment - Toward a Sustainable Future, Cambridge University Press and the International Institute for Applied Systems Analysis, Cambridge, UK, New York, NY, USA and Laxenburg, Austria.

GFATM (2001), "The Framework Document," Global Fund to Fight AIDS, Tuberculosis and Malaria, Geneva.

(2013), "The Global Fund's New Funding Model," Global Fund to Fight AIDS, Tuberculosis and Malaria, Geneva.

- (2015a), "The Right Side of the Tipping Point For AIDS, Tuberculosis and Malaria. Investment Case for the Global Fund's 2017-2019 Replenishment," Geneva.

(2015b), "Global Fund Results Report 2015," Global Fund to Fight AIDS, Tuberculosis and Malaria, Geneva.

(2016a), "List of Successful Appeals in Previous Rounds," Global Fund to Fight AIDS, Tuberculosis and Malaria, Geneva.

(2016b), Global Fund Board Decisions, http://www.theglobalfund.org/en/board/decisions/, accessed December 7, 2016.

(2016c), Global Fund Implementation Periods Database, http://web-api.theglobalfund.org/odata/grants/GrantAgreementImplementationPeriods, accessed December 7, 2016.

(2016d), Global Fund Grant Agreement Progress Updates Database, http://webapi.theglobalfund.org/odata/grants/GrantAgreementProgressUpdates, accessed December 7, 2016.

(2016e), Global Fund Grant Agreements Database, http://web-api.theglobalfund.org/odata/grants/GrantAgreements), accessed December 7, 2016.

(2016f), Global Fund Contact Organizations Database, http://web-api.theglobalfund.org/odata/contacts/ContactOrganizations, accessed December 7, 2016. 
GFF (2015), "Business Plan for the Global Financing Facility in Support of Every Woman Every Child," Global Financing Facility, Washington D.C.

Glassman, A., V.Y. Fan, and M. Over (2013), "More Health for the Money: Putting Incentives to Work for the Global Fund and Its Partners," Working Group on Value for Money in Global Health, Center for Global Development, Washington, D.C.

Global Humanitarian Assistance (2015), "Global Humanitarian Assistance Report 2015," Global Humanitarian Assistance, Geneva.

Greenhill, R., C. Hoy, P. Carter, and M. Manuel (2015), "Financing the future: How international public finance should fund a global social compact to eradicate poverty," Overseas Development Institute, London.

Gupta, J., A. Persson, L. Olsson, J. Linnerooth-Bayer, N. van der Grijp, A. Jerneck, R.J.T. Klein, M. Thompson, and A. Patt (2010), "Mainstreaming climate change in development co-operation policy: conditions for success," in: Making Climate Change Work for Us: European Perspectives on Adaptation and Mitigation Strategies, M. Hulme and H. Neufeldt (Eds.), Cambridge University Press, Cambridge, UK.

Hafner, T., and J. Shiffman (2013), "The emergence of global attention to health systems strengthening," Health Policy and Planning, 28(1), 41-50.

Hagen-Zanker, J., and A. McCord (2011), "The feasibility of financing sectoral development targets," ODI Project Briefing, No 55, Overseas Development Institute, London.

Halpern, B.S., C. Longo, D. Hardy, K.L. McLeod, J.F. Samhouri, S.K. Katona, K. Kleisner, S.E. Lester, J. O'Leary, M. Ranelletti, A.A. Rosenberg, C. Scarborough, E.R. Selig, B.D. Best, D.R. Brumbaugh, F.S. Chapin, L.B. Crowder, K.L. Daly, S.C. Doney, C. Elfes, M.J. Fogarty, S.D. Gaines, K.I. Jacobsen, L.B. Karrer, H.M. Leslie, E. Neeley, D. Pauly, S. Polasky, B. Ris, K. St Martin, G.S. Stone, U.R. Sumaila, and D. Zeller (2012), "An index to assess the health and benefits of the global ocean," Nature, 488(7413), 615-620.

Hamilton, K., and M. Clemens (1999), "Genuine Savings Rates in Developing Countries," World Bank Economic Review, 13(2), 333-356.

Harmer, A., N. Spicer, J. Aleshkina, D. Bogdan, K. Chkhatarashvili, G. Murzalieva, N. Rukhadze, A. Samiev, and G. Walt (2012), "Has Global Fund support for civil society advocacy in the Former Soviet Union established meaningful engagement or 'a lot of jabber about nothing'?," Health Policy and Planning, 299-308.

Hausmann, R., D. Rodrik, and A. Velasco (2008), "Growth diagnostics," in: The Washington Consensus Reconsidered: Towards a New Global Governance, Oxford University Press, New York, pp. 324-355.

Häyhä, T., P.L. Lucas, D.P. van Vuuren, S.E. Cornell, and H. Hoff (2016), "From Planetary Boundaries to national fair shares of the global safe operating space How can the scales be bridged?," Global Environmental Change, 40, 60-72.

HCSS (2015), "Climate change vulnerability monitor," The Hague Centre for Strategic Studies, The Hague.

Helliwell, J.F., H. Huang, and S. Wang (2016a), "World Happiness Report. Chapter 2: The Distribution of World Happiness," Sustainable Development Solutions Network, New York.

Helliwell, J.F., R. Layard, and J.D. Sachs (2016b), "World Happiness Report 2016 (Update)," Sustainable Development Solutions Network, New York.

HLIRP (2011), "Turning the Page from Emergency to Sustainability: The Final Report of the High-Level Independent Review Panel on Fiduciary Controls and Oversight Mechanisms of the Global Fund to Fight AIDS, Tuberculosis and Malaria," High-Level Independent Review Panel on Fiduciary Controls and Oversight Mechanisms of the Global Fund to Fight AIDS, Tuberculosis and Malaria, Geneva. 
Hsu, A., D.C. Esty, M.A. Levy, and A. de Sherbinin (2016), "2016 Environmental Performance Index (EPI)," Yale Center for Environmental Law \& Policy, New Haven, CT.

Hughes, G., P. Chinowsky, and K. Strzepek (2010), "The Costs of Adapting to Climate Change for Infrastructure," Discussion Paper No. 2, World Bank, Washington, D.C.

Hulme, M., and H. Neufeldt (2010), Making climate change work for us, Cambridge University Press, Cambridge.

Hutton, G. (2015), "Benefits and Costs of the Water Sanitation and Hygiene Targets for the Post-2015 Development Agenda," Copenhagen Consensus Center, Copenhagen.

IAEG-SDGs (2016a), "Update on the work to finalize the proposals for the global indicators for the Sustainable Development Goals," Background document, Interagency Expert Group on SDG Indicators, New York.

- (2016b), "Provisional Proposed Tiers for Global SDG Indicators as of March 24, 2016," Inter-agency Expert Group on SDG Indicators, New York.

ICESDF (2014), "Report of the Intergovernmental Committee of Experts on Sustainable Development Financing," United Nations, New York.

ICPR (2016), "World Prison Population List 11th Edition," Institute for Criminal Policy Research, London.

ICSU, and ISSC (2015), "Review of the Sustainable Development Goals: The Science Perspective," International Council for Science, International Social Science Council, Paris.

IDA (2013), "The Demand for IDA17 Resources and the Strategy for their Effective Use," International Development Association, Washington, D.C.

IEA (2011), "World Energy Outlook 2011," International Energy Agency, Paris.

— (2012), "Energy Technology Perspectives," International Energy Agency, Paris.

— (2016), "CO2 Emissions from Fuel Combustion (2016 Edition)," International Energy Agency, Paris.

IEP (2016), "Global Peace Index," Institute for Economics and Peace, Sydney.

iERG (2014), "Every Woman, Every Child: A Post-2015 Vision," Independent Expert Review Group on Information and Accountability for Women's and Children's Health, Geneva.

IHME (2016), Development Assistance for Health Database 1990-2015, http://ghdx.healthdata.org/record/development-assistance-health-database1990-2015, accessed December 7, 2016.

ILO (2014), "World Social Protection Report," International Labor Organization, Geneva.

(2016a), Labor force participation rate, total (\% of total population ages $15+$ ) (modeled ILO estimate) | Data, http://data.worldbank.org/indicator/SL.TLF.CACT.ZS, accessed December 7, 2016.

(2016b), Unemployment, total (\% of total labor force) (modeled ILO estimate) | Data, http://data.worldbank.org/indicator/SL.UEM.TOTL.ZS, accessed December 7, 2016.

IMF (2008), "The Macroeconomics of Scaling-Up Aid: The cases of Benin, Niger, and Togo," International Monetary Fund, Washington, D.C.

(2015a), "Financial Access Survey," International Monetary Fund, Washington, D.C.

_ (2015b), "The IMF and the Sustainable Development Goals," International Monetary Fund, Washington, D.C.

— (2016), "World Economic Outlook," World Economic Outlook, International Monetary Fund, Washington, DC.

Inniss, L., A. Simcock, A.Y. Ajawin, A.C. Alcala, P. Bernal, H.P. Calumpong, P.E. Araghi, S.O. Green, P. Harris, O.K. Kamara, K. Kohata, E. Marschoff, G. Martin, B.P. Fereira, C. Park, R.A. Payet, J. Rice, A. Rosenberg, R. Ruwa, J.T. 
Tuhumwire, S. Van Gaever, J. Wang, and J.M. Weslawski (2016), "The First Global Integrated Marine Assessment," United Nations, New York.

IPCC (2014), "IPCC Fifth Assessment Report: Climate Change 2014 Synthesis Report," Intergovernmental Panel on Climate Change, Geneva.

IPU (2016), Proportion of seats held by women in national parliaments (\%) | Data, http://data.worldbank.org/indicator/SG.GEN.PARL.ZS, accessed December 7, 2016

ITU (2016), World Telecommunication/ICT Indicators database, http://www.itu.int/en/ITU-D/Statistics/Pages/publications/wtid.aspx, accessed December 7, 2016.

IUCN, and BirdLife International (2017), "IUCN Red List," International Union for Conservation of Nature and Birdlife International, Gland.

Jamison, D.T., L.H. Summers, G. Alleyne, K.J. Arrow, S. Berkley, A. Binagwaho, F. Bustreo, D. Evans, R.G.A. Feachem, J. Frenk, G. Ghosh, S.J. Goldie, Y. Guo, S. Gupta, R. Horton, M.E. Kruk, A. Mahmoud, L.K. Mohohlo, M. Ncube, A. Pablos-Mendez, K.S. Reddy, H. Saxenian, A. Soucat, K.H. Ulltveit-Moe, and G. Yamey (2013), "Global health 2035: a world converging within a generation," The Lancet, 382(9908), 1898-1955.

Johansson, A., Y. Guillemette, F. Murtin, D. Turner, G. Nicoletti, C. de la Maisonneuve, P. Bagnoli, G. Bousquet, and F. Spinelli (2013), "Long-term growth scenarios," OECD, Paris.

Jones, G., R.W. Steketee, R.E. Black, Z.A. Bhutta, S.S. Morris, and Bellagio Child Survival Study Group (2003), "How many child deaths can we prevent this year?," The Lancet, 362(9377), 65-71.

Joshi, D.K., B.B. Hughes, and T.D. Sisk (2015), "Improving Governance for the Post2015 Sustainable Development Goals: Scenario Forecasting the Next 50 years," World Development, 70, 286-302.

Kageni, A., L. Mwangi, C. Mugyenyi, and K. Maclntyre (2015), "Representation and Participation of Key Populations on Country Coordinating Mechanisms (CCMs) in Six Countries in Southern Africa," Aidspan, Nairobi.

Kassebaum, N.J., A. Bertozzi-Villa, M.S. Coggeshall, K.A. Shackelford, C. Steiner, K.R. Heuton, D. Gonzalez-Medina, R. Barber, C. Huynh, D. Dicker, and T. Templin (2014), "Global, regional, and national levels and causes of maternal mortality during 1990-2013: a systematic analysis for the Global Burden of Disease Study 2013," The Lancet, 384(9947), 980-1004.

Katz, I., M.A. Aziz, M. Olszak-Olszewski, R. Komatsu, D. Low-Beer, and R. Atun (2010), "Factors influencing performance of Global Fund-supported tuberculosis grants," The International Journal of Tuberculosis and Lung Disease, 14(9), 1097-1103.

Kerouedan, D. (2010), "Enjeux politiques de l'évaluation à cinq ans du Fonds mondial de lutte contre le sida, la tuberculose et le paludisme," Bulletin de la Société de Pathologie Exotique, 103(2), 119-122.

Kharas, H. (2007), "Trends and issues in development aid," Wolfensohn Center for Development. Brookings Institution, Washington D.C.

— (2014), "Reforming ODA to Increase Development Impact," The Brookings Institution, Washington D.C.

Kharas, H., A. Prizzon, and A. Rogerson (2014), "Financing the post-2015 Sustainable Development Goals," Overseas Development Institute, London.

Kim, J. (2015), Speech by World Bank Group President Jim Yong Kim at the United Nations General Assembly.

Ki-Moon, B., A. Al-Madani, J.M. Barroso, A. Gurria, D. Kaberuka, D. Strauss-Kahn, J. Ping, and R. Zoellick (2008), Achieving the MDGs: Recommendations of the MDG Africa Steering Group, United Nations, New York. 
Klein, R.J., and A. Möhner (2009), "Governance limits to effective global financial support for adaptation," in: Adapting to Climate Change: Thresholds, Values, Governance. W.N. Adger, I. Lorenzoni, K. O'Brien (Eds), Cambridge University Press, Cambridge.

Klein, R.J., and A. Persson (2008), "Financing adaptation to climate change: issues and priorities," ECP Report No. 8, European Climate Platform, Stockholm.

Kroll, C. (2011), Measuring progress and well-being: Achievements and challenges of a new global movement, Friedrich Ebert Foundation, Berlin.

Kroll, C. (2015), "Sustainable Development Goals: Are the rich countries ready?," Bertelsmann Foundation, Gütersloh.

Levin, J. (2015a), "MDG Achievement in Tanzania - Is it possible? MAMS-based analysis," Örebro University School of Business, Örebro, Sweden.

(2015b), "Policy Options Beyond 2015 - Achieving the MDGs in Bangladesh," Örebro University School of Business, Örebro, Sweden.

Lim, S.S., T. Vos, A.D. Flaxman, G. Danaei, K. Shibuya, H. Adair-Rohani, M.A. AlMazroa, M. Amann, H.R. Anderson, K.G. Andrews, M. Aryee, C. Atkinson, L.J. Bacchus, A.N. Bahalim, K. Balakrishnan, J. Balmes, S. Barker-Collo, A. Baxter, M.L. Bell, J.D. Blore, F. Blyth, C. Bonner, G. Borges, R. Bourne, M. Boussinesq, M. Brauer, P. Brooks, N.G. Bruce, B. Brunekreef, C. Bryan-Hancock, C. Bucello, R. Buchbinder, F. Bull, R.T. Burnett, T.E. Byers, B. Calabria, J. Carapetis, E. Carnahan, Z. Chafe, F. Charlson, H. Chen, J.S. Chen, A.T.-A. Cheng, J.C. Child, A. Cohen, K.E. Colson, B.C. Cowie, S. Darby, S. Darling, A. Davis, L. Degenhardt, F. Dentener, D.C. Des Jarlais, K. Devries, M. Dherani, E.L. Ding, E.R. Dorsey, T. Driscoll, K. Edmond, S.E. Ali, R.E. Engell, P.J. Erwin, S. Fahimi, G. Falder, F. Farzadfar, A. Ferrari, M.M. Finucane, S. Flaxman, F.G.R. Fowkes, G. Freedman, M.K. Freeman, E. Gakidou, S. Ghosh, E. Giovannucci, G. Gmel, K. Graham, R. Grainger, B. Grant, D. Gunnell, H.R. Gutierrez, W. Hall, H.W. Hoek, A. Hogan, H.D. Hosgood III, D. Hoy, H. Hu, B.J. Hubbell, S.J. Hutchings, S.E. Ibeanusi, G.L. Jacklyn, R. Jasrasaria, J.B. Jonas, H. Kan, J.A. Kanis, N. Kassebaum, N. Kawakami, Y.-H. Khang, S. Khatibzadeh, J.-P. Khoo, C. Kok, F. Laden, R. Lalloo, Q. Lan, T. Lathlean, J.L. Leasher, J. Leigh, Y. Li, J.K. Lin, S.E. Lipshultz, S. London, R. Lozano, Y. Lu, J. Mak, R. Malekzadeh, L. Mallinger, W. Marcenes, L. March, R. Marks, R. Martin, P. McGale, J. McGrath, S. Mehta, Z.A. Memish, G.A. Mensah, T.R. Merriman, R. Micha, C. Michaud, V. Mishra, K.M. Hanafiah, A.A. Mokdad, L. Morawska, D. Mozaffarian, T. Murphy, M. Naghavi, B. Neal, P.K. Nelson, J.M. Nolla, R. Norman, C. Olives, S.B. Omer, J. Orchard, R. Osborne, B. Ostro, A. Page, K.D. Pandey, C.D. Parry, E. Passmore, J. Patra, N. Pearce, P.M. Pelizzari, M. Petzold, M.R. Phillips, D. Pope, C.A. Pope III, J. Powles, M. Rao, H. Razavi, E.A. Rehfuess, J.T. Rehm, B. Ritz, F.P. Rivara, T. Roberts, C. Robinson, J.A. Rodriguez-Portales, I. Romieu, R. Room, L.C. Rosenfeld, A. Roy, L. Rushton, J.A. Salomon, U. Sampson, L. Sanchez-Riera, E. Sanman, A. Sapkota, S. Seedat, P. Shi, K. Shield, R. Shivakoti, G.M. Singh, D.A. Sleet, E. Smith, K.R. Smith, N.J. Stapelberg, K. Steenland, H. Stöckl, L.J. Stovner, K. Straif, L. Straney, G.D. Thurston, J.H. Tran, R. Van Dingenen, A. van Donkelaar, J.L. Veerman, L. Vijayakumar, R. Weintraub, M.M. Weissman, R.A. White, H. Whiteford, S.T. Wiersma, J.D. Wilkinson, H.C. Williams, W. Williams, N. Wilson, A.D. Woolf, P. Yip, J.M. Zielinski, A.D. Lopez, C.J. Murray, and M. Ezzati (2012), "A comparative risk assessment of burden of disease and injury attributable to 67 risk factors and risk factor clusters in 21 regions, 1990-2010: a systematic analysis for the Global Burden of Disease Study 2010," The Lancet, 380(9859), 2224-2260.

Lind, N. (2014), "Better Life Index," in: Encyclopedia of Quality of Life and Well-Being Research, Springer, New York, pp. 381-382. 
Lofgren, H., M. Cicowiez, and C. Diaz-Bonilla (2013), "MAMS - A computable general equilibrium model for developing country strategy analysis," in: Handbook of Computable General Equilibrium Modeling, vol. 1, Elsevier, The Hague, pp. 159-276.

Lofgren, H., and C. Diaz-Bonilla (2008), "MAMS: An Economy-wide Model for Analysis of MDG Country Strategies: Technical Documentation," World Bank, Washington D.C.

Lu, C., C.M. Michaud, K. Khan, and C.J. Murray (2006), "Absorptive capacity and disbursements by the Global Fund to Fight AIDS, Tuberculosis and Malaria: analysis of grant implementation," The Lancet, 368(9534), 483-488.

Lu, C., M.T. Schneider, P. Gubbins, K. Leach-Kemon, D. Jamison, and C.J. Murray (2010), "Public financing of health in developing countries: a cross-national systematic analysis," The Lancet, 375(9723), 1375-1387.

Lu, Y., N. Nakicenovic, M. Visbeck, and A.-S. Stevance (2015), "Policy: Five priorities for the UN Sustainable Development Goals-Comment," Nature, 520(7548), 432-433.

Macro International (2009), "The Five-Year Evaluation of the Global Fund to Fight AIDS, Tuberculosis, and Malaria Synthesis of Study Areas 1, 2 and 3," Macro International, Geneva.

McArthur, J.W. (2013), "Own the goals: What the Millennium Development Goals have accomplished," Foreign Affairs, 92, 152.

_ (2014), "The origins of the millennium development goals," SAIS Review of International Affairs, 34(2), 5-24.

McArthur, J.W., and K. Rasmussen (2017), "Change of pace: Accelerations and advances during the Millennium Development Goal era," Brookings Global Economy and Development Working Paper 97, Brookings Institution, Washington D.C.

McCollum, D., Y. Nagai, K. Riahi, G. Marangoni, K. Calvin, R. Pietzcker, J. Van Vliet, and B. Van der Zwaan (2013), "Energy investments under climate policy: a comparison of global models," Climate Change Economics, 4(4), 1340010.

McCollum, D.L., V. Krey, and K. Riahi (2012), "Beyond Rio: Sustainable energy scenarios for the 21st century," Natural Resources Forum, 36(4), 215-230.

McGray, H., A. Hammill, R. Bradley, E.L. Schipper, and J.-E. Parry (2007), Weathering the storm: Options for framing adaptation and development, World Resources Institute, Washington D.C.

McKinsey (2005), "Global Health Partnerships: Assessing Country Consequences," McKinsey \& Company, Geneva.

— (2008), "Independent Evaluation of the Stop TB Partnership," McKinsey \& Company, Geneva.

Messner, S., and M. Strubegger (1995), “User's Guide for MESSAGE III," No. WP-9569, International Institute for Applied Systems Analysis, Laxenburg, Austria.

Miller, T. (2015), Index of economic freedom, Wall Street Journal, New York.

Miller, T., A.B. Kim, J. DeMint, J.M. Roberts, B. Riley, T. Whiting, M. Giugale, F. Schneider, A.F. Abbott, and S. Singham (2016), "2016 Index of Economic Freedom: Promoting Economic Opportunity and Prosperity," The Heritage Foundation in partnership with the Wall Street Journal, Washington D.C.

Minghui, R., F. Scano, C. Sozi, and B. Schwartländer (2015), "The Global Fund in China: success beyond the numbers," The Lancet Global Health, 3(2), e75e77.

Mishra, P., and D.L. Newhouse (2007), "Health aid and infant mortality," IMF Working Paper No. 07/100, IMF, Washington D.C.

Mongardini, J., and I. Samake (2009), "The Macroeconomics of Scaling Up Aid: The Gleneagles Initiative for Benin," Working Paper No. WP/09/115, International Monetary Fund, Washington, D.C. 
Moreno-Serra, R., and P. Smith (2012), "Does progress towards universal health coverage improve population health?," The Lancet, 380(9845), 917-923.

Müller, B. (2008), "International adaptation finance: the need for an innovative and strategic approach," Economic Working Paper 42, Oxford Institute for Energy Studies, Oxford, UK.

Müller, C. (2009), "Climate change impact on Sub-Saharan Africa: an overview and analysis of scenarios and models," Discussion Paper 3/2009, Deutsches Institut für Entwicklungspolitik, Bonn.

Muller, M. (2007), "Adapting to climate change: water management for urban resilience," Environment and Urbanization, 19(1), 99-113.

Murray, C.J. (2015), "Global, regional, and national age-sex specific all-cause and cause-specific mortality for 240 causes of death, 1990-2013: a systematic analysis for the Global Burden of Disease Study 2013," The Lancet, 385(9963), $117-171$.

Murray, C.J., and R. Chambers (2015), "Keeping score: fostering accountability for children's lives," The Lancet, 386(9988), 3-5.

Mutabingwa, T.K. (2005), "Artemisinin-based combination therapies (ACTs): Best hope for malaria treatment but inaccessible to the needy!," Acta Tropica, 95(3), 305-315.

Nahlen, B.L., and D. Low-Beer (2007), "Building to Collective Impact: The Global Fund Support for Measuring Reduction in the Burden of Malaria," The American Journal of Tropical Medicine and Hygiene, 77(6 Suppl), 321-327.

NCEC (2014), "Technical Note: Infrastructure investment needs of a low-carbon scenario," New Climate Economy Commission, Washington, D.C.

Nelson, D., M. Hervé-Mignucci, A. Goggins, S.J. Szambelan, and J. Zuckerman (2014), "Moving to a low-carbon economy: the financial impact of the low-carbon transition," Climate Policy Initiative, San Francisco.

Nicolai, S., C. Hoy, T. Berliner, and T. Aedy (2015), "Projecting progress: reaching the SDGs by 2030," Overseas Development Institute, London.

Nilsson, M., D. Griggs, and M. Visbeck (2016), "Policy: Map the interactions between Sustainable Development Goals," Nature, 534(7607), 320-322.

Noor, A.M., J.J. Mutheu, A.J. Tatem, S.I. Hay, and R.W. Snow (2009), "Insecticidetreated net coverage in Africa: mapping progress in 2000-07," The Lancet, 373(9657), 58-67.

Nordhaus, W.D. (2008), A Question of Balance: Economic Modeling of Global Warming, Yale University Press, New Haven, CT.

Norwegian Ministry of Foreign Affairs (2013), "The Global Fund to Fight AIDS, Tuberculosis and Malaria," Norwegian Ministry of Foreign Affairs, Oslo.

Noy, I. (2009), "The macroeconomic consequences of disasters," Journal of Development economics, 88(2), 221-231.

Ocean Health Index (2016), Ocean Health Index Data, http://data.oceanhealthindex.org/data-and-downloads, accessed December 13, 2016.

OCHA (2015), "Global Humanitarian Overview 2015. A consolidated appeal to support people affected by disaster and conflict," Office for the Coordination of Humanitarian Affairs, United Nations, New York.

ODI, ECDPM, GDI, University of Athens, and Southern Voice Network (2015), "European Report on Development 2015. Combining finance and policies to implement a transformative post-2015 development agenda," Overseas Development Institute (ODI), European Centre for Development Policy Management (ECDPM), German Development Institute (GDI), University of Athens, Southern Voice Network, Brussels.

OECD (2006), "Infrastructure to 2030: Telecom, Land Transport, Water and Electricity," Organisation for Economic Co-operation and Development, Paris.

(2008), "The Paris Declaration on Aid Effectiveness and the Accra Agenda for Action," OECD, Paris. 
(2009), "Development Cooperation Report 2009," Organisation for Economic Cooperation and Development, Paris.

(2012), "PISA 2012 Results in Focus. What 15-year-olds know and what they can do with what they know," OECD, Paris.

(2013), OECD Guidelines on Measuring Subjective Well-being, OECD, Paris.

(2014), "Development Co-operation Report 2014," OECD, Paris.

(2015a), How's Life? 2015, OECD, Paris.

(2015b), Statistics on resource flows to developing countries, http://www.oecd.org/dac/stats/statisticsonresourceflowstodevelopingcountries.htm, accessed December 7, 2016.

(2016a), "Measuring Distance to the SDGs Targets: a pilot assessment of where OECD countries stand," OECD, Paris.

(2016b), OECD Statistics, http://stats.oecd.org/, accessed December 7, 2016.

(2016c), Development Co-operation Report 2016, OECD, Paris.

- (2017a), PISA Database, http://www.oecd.org/pisa/, accessed January 4, 2017.

- (2017b), "Measuring distance to the SDG targets. An assessment of where OECD countries stand," OECD, Paris.

OECD Better Life Initiative (2011), "Your Better Life Index," OECD, Paris.

OECD, and CPI (2015), "Climate Finance in 2013-14 and the USD 100 billion goal," OECD and Climate Policy Initiative, Paris.

OECD, IEA, and World Bank (2017), Renewable energy consumption ( $\%$ of total final energy consumption) | Data, http://data.worldbank.org/indicator/EG.FEC.RNEW.ZS, accessed January 4, 2017.

OECD, and JRC (2016), Handbook on Constructing Composite Indicators: Methodology and User Guide, OECD, Joint Research Committee, Paris.

Osman-Elasha, B., and T. Downing (2007), "Lessons learned in preparing national adaptation programmes of action in Eastern and Southern Africa," European Capacity Building Initiative, Oxford.

Pachauri, S., B.J. van Ruijven, Y. Nagai, K. Riahi, D.P. van Vuuren, A. Brew-Hammond, and N. Nakicenovic (2013), "Pathways to achieve universal household access to modern energy by 2030," Environmental Research Letters, 8(2), 024015.

Pandey, K. (2010), "Costs of Adapting to Climate Change for Human Health in Developing Countries," Discussion Paper Number 11, World Bank, Washington D.C.

Parry, M.L., N. Arnell, P. Berry, D. Dodman, S. Frankhauser, C. Hope, S. Kovats, R. Nicholls, R. Satterhwaite, R. Tiffin, and T. Wheeler (2009), Assessing the costs of adaptation to climate change: a review of the UNFCCC and other recent estimates, International Institute for Environment and Development and Grantham Institute on Climate Change, London.

Patel, P., R. Cummings, and B. Roberts (2015), "Exploring the influence of the Global Fund and the GAVI Alliance on health systems in conflict-affected countries," Conflict and Health, 9(7).

PBL (2015), "IMAGE 3.0 Documentation," Netherlands Environmental Assessment Agency (PBL), The Hague.

Pearce, D.W., and G.D. Atkinson (1993), "Capital theory and the measurement of sustainable development: an indicator of 'weak' sustainability," Ecological economics, 8(2), 103-108.

PEPFAR (2016), PEPFAR Framework Countries, http://www.pepfar.gov/countries/frameworks/, accessed December 7, 2016.

Perelman, M.I. (2000), "Tuberculosis in Russia," The International Journal of Tuberculosis and Lung Disease, 4(12), 1097-1103.

Piot, P., M. Kazatchkine, M. Dybul, and J. Lob-Levyt (2009), "AIDS: lessons learnt and myths dispelled," The Lancet, 374(9685), 260-263.

Poate, D., P. Balogun, and K. Attawell (2009), "UNAIDS. Second Independent Evaluation 2002-2008," ITAD and hlsp, Geneva. 
Prakash, M., K. Teksoz, J. Espey, J.D. Sachs, and M. Shank (2017a), "Achieving a Sustainable America. The U.S. Cities Sustainable Development Goals Index. Draft for public consultation," Sustainable Development Solutions Network, New York.

Prakash, M., K. Teksoz, J. Espey, J.D. Sachs, M. Shank, and G. Schmidt-Traub (2017b), "Achieving a Sustainable Urban America. The U.S. Cities Sustainable Development Goals Index 2017," Sustainable Development Solutions Network, New York.

Prati, A., S. Sahay, and T. Tressel (2003), "Is There a Case for Sterilizing Foreign Aid Inflows?," Working Paper, International Monetary Fund, Washington D.C.

Project Catalyst (2009), "Adaptation to Climate Change: Potential Costs and Choices for a Global Agreement. Findings of the Adaptation Working Group of Project Catalyst," Climate Works Foundation and McKinsey \& Co., London.

PublishWhatYouFund (2016), "Aid Transparency Index 2016," PublishWhatYouFund, London.

Radelet, S., and B. Siddiqi (2007), "Global Fund grant programmes: an analysis of evaluation scores," The Lancet, 369(9575), 1807-1813.

Reddy, S., and A. Heuty (2006), "Achieving the Millennium Development Goals: What's wrong with existing analytical models?," DESA working paper No. 30, UN Department of Economic and Social Affairs, New York.

Rickels, W., M. Quaas, and M. Visbeck (2014), "How healthy is the human-ocean system?," Environment Research Letters, 9(4), 044013.

Rockström, J., W. Steffen, K. Noone, WAsa Persson, F.S. Chapin III, E. Lambin, T. Lenton, M. Scheffer, C. Folke, H.J. Schellnhuber, B. Nykvist, C.A. de Wit, T. Hughes, S. van der Leeuw, H. Rodhe, S. Sörlin, P.K. Snyder, R. Costanza, U. Svedin, M. Falkenmark, L. Karlberg, R.W. Corell, V.J. Fabry, J. Hansen, B. Walker, D. Liverman, K. Richardson, P. Crutzen, and J.A. Foley (2009), "Planetary boundaries: exploring the safe operating space for humanity," Ecology and society, 14(2).

Roll Back Malaria Partnership (2008), "The Global Malaria Action Plan. For a malariafree world," Roll Back Malaria Partnership, Geneva.

Roth, G.A., G. Nguyen, M.H. Forouzanfar, A.H. Mokdad, M. Naghavi, and C.J.L. Murray (2015), "Estimates of global and regional premature cardiovascular mortality in 2025," Circulation, 132, 1270-1282.

Sachs, J.D. (2000), HIV non-intervention: A costly option. A new framework for globalization, Durban.

- (2005), The end of poverty: How we can make it happen in our lifetime, Penguin UK, London.

Sachs, J.D. (2015), The age of sustainable development, Columbia University Press, New York.

Sachs, J.D., J.W. McArthur, G. Schmidt-Traub, M. Kruk, C. Bahadur, M. Faye, and G. McCord (2004a), "Ending Africa's Poverty Trap," Brookings Papers on Economic Activity, 2004(1), 117-240.

_ (2004b), "Millennium Development Goals Needs Assessments for Ghana, Tanzania, and Uganda. Background Paper to "Ending Africa's Poverty Trap," Working Paper, UN Millennium Project, New York.

Sachs, J.D., and G. Schmidt-Traub (2017), "Global Fund lessons for Sustainable Development Goals," Science, 355(6333).

Sachs, J.D., G. Schmidt-Traub, and D. Durand-Delacre (2016a), "Preliminary Sustainable Development Goal (SDG) Index and Dashboards," Sustainable Development Solutions Network, New York.

Sachs, J.D., G. Schmidt-Traub, C. Kroll, D. Durand-Delacre, and K. Teksoz (2017), "SDG Index and Dashboards Report 2017. Global Responsibilities - International Spillovers in Achieving the Goals," Bertelsmann Stiftung and Sustainable Development Solutions Network, Gütersloh and New York. 
Sachs, J.D., G. Schmidt-Traub, C. Kroll, K. Teksoz, and D. Durand-Delacre (2016b), "Sustainable Development Goals Index and Dashboards - Global Report," Bertelsmann Stiftung and Sustainable Development Solutions Network, Gütersloh and New York.

Sachs, J.D., G. Schmidt-Traub, and J. Williams (2016c), "Pathways to zero emissions," Nature Geoscience, 9(11), 799-801.

Samb, B., T. Evans, M. Dybul, R. Atun, J.-P. Moatti, S. Nishtar, A. Wright, F. Celletti, J. Hsu, J.Y. Kim, R. Brugha, A. Russell, and C. Etienne (2009), "An assessment of interactions between global health initiatives and country health systems," The Lancet, 373(9681), 2137-2169.

Savedoff, W.D. (2012), "Transitions in Health Financing and Policies for Universal Health Coverage," Results for Development Institute, Washington, D.C.

Schmidt-Traub, G. (2009), "Climate Change: A Call to Action for African Leaders," Discussion Paper, Africa Progress Panel, African Development Bank, United Nations Economic Commission for Africa, United Nations Environment Program, Geneva.

Schmidt-Traub, G. (2015), "Investment Needs to Achieve the Sustainable Development Goals," SDSN Working Paper, Sustainable Development Solutions Network, Paris.

(2017), "A Long-Term Transformation Pathways Initiative (LTTPI) for the G20," G20 Insights, Kiel.

Schmidt-Traub, G., C. Kroll, K. Teksoz, D. Durand-Delacre, and J.D. Sachs (2017), "National baselines for the Sustainable Development Goals assessed in the SDG Index and Dashboards," Nature Geoscience, 10(8), 547-555.

Schmidt-Traub, G., and J.D. Sachs (2015), "Financing for Sustainable Development: Implementing the SDGs through Effective Investment Strategies and Partnerships," SDSN Working Paper, Sustainable Development Solutions Network, Paris and New York.

Schmidt-Traub, G., and B. Wylie (2009), "Kick-starting Africa's Carbon Markets. The Potential for Programmatic CDM," Africa Progress Panel, Geneva.

Schwab, K., and X. Sala-i-Martín (2016), "The Global Competitiveness Report 20162017," World Economic Forum, Geneva.

Schwartländer, B., I. Grubb, and J. Perriëns (2006), "The 10-year struggle to provide antiretroviral treatment to people with HIV in the developing world," The Lancet, 368(9534), 541-546.

SDSN (2013), "An Action Agenda for Sustainable Development," Leadership Council of the Sustainable Development Solutions Network, New York.

— (2015a), "Indicators and a Monitoring Framework for Sustainable Development Goals: Launching a data revolution for the SDGs," Sustainable Development Solutions Network, Paris and New York.

(2015b), "Key Elements of a Successful Addis Ababa Accord on Financing for Sustainable Development," Working paper, Leadership Council of the Sustainable Development Solutions Network, New York.

SDSN, and IDDRI (2015), "Pathways to Deep Decarbonization 2015 Report," Sustainable Development Solutions Network, The Institute for Sustainable Development and International Relations, Paris and New York.

SE4All (2017a), Access to electricity (\% of population) | Data, http://data.worldbank.org/indicator/EG.ELC.ACCS.ZS, accessed January 4, 2017.

(2017b), Access to non-solid fuel (\% of population) | Data, http://data.worldbank.org/indicator/EG.NSF.ACCS.ZS, accessed January 4, 2017.

Sea Around Us (2016), Sea Around Us: Tools and Data, http://www.seaaroundus.org/data/\#/eez, accessed December 7, 2016. 
Selomane, O., B. Reyers, R. Biggs, H. Tallis, and S. Polasky (2015), "Towards integrated social-ecological sustainability indicators: Exploring the contribution and gaps in existing global data," Ecological Economics, 118, 140-146.

Shakow, A. (2006), "Global Fund - World Bank HIVIAIDS Programs Comparative Advantage Study," World Bank and Global Fund to Fight AIDS, Tuberculosis and Malaria, Washington D.C. and Geneva.

Shapiro, S.S., and M.B. Wilk (1965), "An Analysis of Variance Test for Normality (Complete Samples)," Biometrika, 52(3/4), 591-611.

Simon, J., K. Yeboah-Antwi, M. Bryant, M.B. Djara, and M.I. Brooks (2013), "External Evaluation of the Roll Back Malaria (RBM) Partnership 2009-2013," Boston University, Center for Global Health and Development, Boston.

Soeters, R. (2013), "Why is it so difficult for the Global Fund to show value for money?," The Lancet Global Health, 1(3), e123-e124.

Sridhar, D. (2016), "Making the SDGs useful: a Herculean task," The Lancet, 388(10053), 1453-1454.

Steer, L., and K. Smith (2015), "Financing Education. Opportunities for International Action," Brookings Institution, Washington D.C.

Steffen, W., K. Richardson, J. Rockström, S.E. Cornell, I. Fetzer, E.M. Bennett, R. Biggs, S.R. Carpenter, W. de Vries, C.A. de Wit, C. Folke, D. Gerten, J. Heinke, G.M. Mace, L.M. Persson, V. Ramanathan, B. Reyers, and S. Sörlin (2015), "Planetary boundaries: Guiding human development on a changing planet," Science, 347(6223), 1259855.

Stern, N. (2007), "Stern Review: The Economics of Climate Change," HM Treasury, London.

(2009a), "Possibilities for Africa in global action on climate change," Grantham Institute on Climate Change and the Environment, London School of Economics, London.

- (2009b), A Blueprint for a Greener Planet, The Bodley Head, London.

Stiglitz, J., A. Sen, and J.P. Fitoussi (2009), "Report by the Commission on the Measurement of Economic Performance and Social Progress," Commission on the Measurement of Economic Performance and Social Progress, Paris.

Stop TB Partnership (2000), "Global Plan to Stop TB 2001-2005," Stop TB Partnership, Geneva.

(2015), "The Paradigm Shift 2016-2020. Global Plan to End TB," Stop TB Partnership, Geneva.

Stover, J., E.L. Korenromp, M. Blakley, R. Komatsu, K. Viisainen, L. Bollinger, and R. Atun (2011), "Long-Term Costs and Health Impact of Continued Global Fund Support for Antiretroviral Therapy," PLoS One, 6(6), e21048.

Temple, J.R.W. (2010), "Aid and Conditionality," in: Handbook of Development Economics, vol. 5, Elsevier, Amsterdam, pp. 4415-4523.

The Boston Globe (2001), "Interview with Andrew Natsios, Administrator of USAID," The Boston Globe.

Tol, R.S., and G.W. Yohe (2007), "The weakest link hypothesis for adaptive capacity: an empirical test," Global Environmental Change, 17(2), 218-227.

Tollefson, J., and N. Gilbert (2012), "Rio report card," Nature, 486(7401), 20-23.

Transparency International (2015), Corruption Perceptions Index 2015, https://www.transparency.org/cpi2015\#results-table, accessed December 13, 2016.

Triponel, A.F. (2010), "Global Fund to Fight Aids, Tuberculosis and Malaria: A New Legal and Conceptual Framework for Providing International Development Aid," SSRN Scholarly Paper No. ID 1558088, Social Science Research Network, Rochester, NY.

TRP (2009), "Report of the Technical Review Panel and the Secretariat on Round 9 Proposals," Global Fund to Fight AIDS, Tuberculosis and Malaria, Geneva. 
UN Millennium Project (2004), "Millennium Development Goals Needs Assessment: Background Paper to 'Ending Africa's Poverty Trap'," UN Millennium Project, New York.

_ (2005), "Investing in Development: A Practical Plan to Achieve the MDGs," Earthscan, New York.

UN Statistics Division (2017), SDG Indicators and Targets, https://unstats.un.org/sdgs/indicators/indicators-list/, accessed May 20, 2017.

UN Women (2015), "Progress of the World's Women 2015-2016: Transforming Economies, Realizing Rights," United Nations Entity for Gender Equality and the Empowerment of Women, New York.

UNAIDS (2014), "90-90-90. An ambitious treatment target to help end the AIDS epidemic," UNAIDS, Geneva.

UNCCD (2009), "Climate Change in the African Drylands. Options and Opportunities for Adaptation and Mitigation," United Nations Convention to Combat Desertification, Nairobi.

UNCTAD (2014), "World Investment Report 2014. Investing in the SDGs: An Action Plan," United Nations Conference on Trade and Development, Geneva.

UNDESA (2017), Family Planning - Model, http://www.un.org/en/development/desa/population/theme/family-planning/cp_model.shtml, accessed January $4,2017$.

UNDP (2007), "Human Development Report 2007," United Nations Development Programme, New York.

_ (2015), "Human Development Report. Work for human development," United Nations Development Programme, New York.

(2016a), "Human Development Report. Human Development for Everyone," United Nations Development Programme, New York.

(2016b), Expected years of schooling, males (years), http://hdr.undp.org/en/content/expected-years-schooling-males-years, accessed December 7, 2016.

(2017), Adolescent fertility rate (births per 1,000 women ages 15-19) | Data, http://data.worldbank.org/indicator/SP.ADO.TFRT, accessed January 4, 2017.

UNESCO (2013), "Education for All Global Monitoring Report: Teaching and Learning - Achieving Quality for All," United Nations Educational, Scientific and Cultural Organization, Paris.

(2015a), "Education for All Global Monitoring Report: The Cost of Reaching New Targets by 2030," UNESCO Policy Paper No. 18, United Nations Educational, Scientific and Cultural Organization, Paris.

(2015b), "Education for All Global Monitoring Report: Education for All 2000-2015 - Achievements and Challenges," United Nations Educational, Scientific and Cultural Organization, Paris.

(2017), UIS.Stat, http://data.uis.unesco.org/, accessed January 4, 2017.

UNFCCC (2007), "Investment and Financial Flows to Address Climate Change," United Nations Framework Convention on Climate Change, Bonn, Germany.

- (2015), "Paris Climate Agreement," United Nations Framework Convention on Climate Change Conference of the Parties, Paris.

UNICEF (2015), "Child Labour," United Nations Children's Emergency Fund.

- (2016), Birth registration | Data, http://data.unicef.org/topic/child-protection/birthregistration/, accessed December 13, 2016.

UNICEF, WHO, and World Bank (2017a), Joint child malnutrition estimates - Levels and trends (2017 edition), http://www.who.int/nutgrowthdb/estimates2016/en/, accessed January 4, 2017.

UNICEF, WHO, World Bank, and UN DESA Population Division (2017b), Mortality rate, under-5 (per 1,000 live births) | Data, http://data.worldbank.org/indicator/SH.DYN.MORT, accessed January 4, 2017. 
(2017c), Mortality rate, neonatal (per 1,000 live births) | Data, http://data.worldbank.org/indicator/SH.DYN.NMRT, accessed January 4, 2017.

United Nations (ed.) (1994), Agenda 21: Programme of action for sustainable development, 2. print, United Nations Conference on Environment and Development, New York, NY.

(2000), "Millennium Declaration: Resolution adopted by the General Assembly. 55/2," United Nations, New York.

(2001), "Secretary-General proposes Global Fund for Fight Against HIVIAIDS and other Infectious Diseases at African Leaders Summit," United Nations, New York.

(2002), "Financing for Development: Monterrey Consensus of the International Conference on Financing for Development," United Nations, New York.

(2012a), "The Future We Want. Outcome document of the United Nations Conference on Sustainable Development," United Nations, Rio de Janeiro.

(2012b), "GLAAS 2012 Report: UN Water Global Analysis and Assessment of Sanitation and Drinking Water: The Challenge of Extending and Sustaining Services," United Nations, New York.

(2015a), "Transforming Our World: The 2030 Agenda for Sustainable Development," No. A/RES/70/1, United Nations, New York.

(2015b), "The Addis Ababa Action Agenda of the Third International Conference on Financing for Development," United Nations, Addis Ababa.

(2016a), "The Sustainable Development Goals Report 2016," United Nations, New York.

(2016b), "Inputs to the 2016 High-level Political Forum on Sustainable Development," United Nations, New York.

_ (2017), "The Sustainable Development Goals Report 2017," United Nations, New York.

UNODC (2016), "Global Study on Homicides," United Nations Office on Drugs and Crime, Vienna.

UNTT (2013), "Financing for sustainable development: Review of global investment requirement estimates," UN Task Team Working Group on Sustainable Development Finance, Mimeo.

UNU-IHDP, and UNEP (2016), Inclusive Wealth Report 2014. Measuring Progress towards Sustainability, Cambridge University Press, Cambridge.

Van de Walle, N. (2005), "Overcoming stagnation in aid-dependent countries," Center for Global Development, Washington D.C.

van Kerkhoff, L., and N.A. Szlezák (2006), "Linking local knowledge with global action: examining the Global Fund to Fight AIDS, Tuberculosis and Malaria through a knowledge system lens," Bulletin of the World Health Organization, 84(8), 629635.

van Kerkhoff, L., and N.A. Szlezák (2010), "The role of innovative global institutions in linking knowledge and action," Proceedings of the National Academy of Sciences, 200900541.

van Vuuren, D.P., M. Kok, P.L. Lucas, A.G. Prins, R. Alkemade, M. van den Berg, L. Bouwman, S. van der Esch, M. Jeuken, T. Kram, and E. Stehfest (2015), "Pathways to achieve a set of ambitious global sustainability objectives by 2050 : Explorations using the IMAGE integrated assessment model," Technological Forecasting and Social Change, 98, 303-323.

Victor, P.A. (1991), "Indicators of sustainable development: some lessons from capital theory," Ecological Economics, 4(3), 191-213.

Vivid Economics (2010), "Promoting Economic Growth When the Climate is Changing," UK Department for International Development, London.

Waage, J., R. Banerji, O. Campbell, E. Chirwa, G. Collender, V. Dieltiens, A. Dorward, P. Godfrey-Faussett, P. Hanvoravongchai, G. Kingdon, A. Little, A. Mills, K. 
Mulholland, A. Mwinga, A. North, W. Patcharanarumol, C. Poulton, V. Tangcharoensathien, and E. Unterhalter (2010), "The Millennium Development Goals: a cross-sectoral analysis and principles for goal setting after 2015," The Lancet, 376(9745), 991-1023.

Waldow, F. (2009), "What PISA Did and Did Not Do: Germany after the 'PISA-shock," European Educational Research Journal, 8(3), 476-483.

Wang, H., M. Naghavi, C. Allen, S.E. Vollset, A.K. Knudsen, A. Hailu, O.F. Norheim, E. Bjertness, A.S. Htet, K. Søreide, and E. Weiderpass (2016), "Global, regional, and national life expectancy, all-cause mortality, and cause-specific mortality for 249 causes of death, 1980-2015: a systematic analysis for the Global Burden of Disease Study 2015," The Lancet, 388(10053), 1459-1544.

Wang, R.-B., Q.-F. Zhang, B. Zheng, Z.-G. Xia, S.-S. Zhou, L.-H. Tang, Q. Gao, L.-Y. Wang, and R.-R. Wang (2014), "Transition from control to elimination: impact of the 10-year global fund project on malaria control and elimination in China," Advances in Parasitology, 86, 289-318.

Ward, P.J., P. Pauw, L.M. Brander, C.J. Jeroen, H. Aerts, and K.M. Strzepek (2010), "Costs of Adaptation Related to Industrial and Municipal Water Supply and Riverine Flood Protection," Discussion Paper No. 6, World Bank, Washington D.C.

Watts, N., W.N. Adger, P. Agnolucci, J. Blackstock, P. Byass, W. Cai, S. Chaytor, T. Colbourn, M. Collins, A. Cooper, P.M. Cox, J. Depledge, P. Drummond, P. Ekins, V. Galaz, D. Grace, H. Graham, M. Grubb, A. Haines, I. Hamilton, A. Hunter, X. Jiang, M. Li, I. Kelman, L. Liang, M. Lott, R. Lowe, Y. Luo, G. Mace, M. Maslin, M. Nilsson, T. Oreszczyn, S. Pye, T. Quinn, M. Svensdotter, S. Venevsky, K. Warner, B. Xu, J. Yang, Y. Yin, C. Yu, Q. Zhang, P. Gong, H. Montgomery, and A. Costello (2015), "Health and climate change: policy responses to protect public health," The Lancet, 386(10006), 1861-1914.

Webster, M., J. Ginnetti, P. Walker, D. Coppard, and R. Kent (2008), The humanitarian costs of climate change, Feinstein International Center, Tufts University, Medford, VA.

WEF (2016), "The Global Competitiveness Report 2015-2016: Full Data Edition," World Economic Forum, Geneva.

Whitmee, S., A. Haines, C. Beyrer, F. Boltz, A.G. Capon, B.F. de Souza Dias, A. Ezeh, H. Frumkin, P. Gong, P. Head, R. Horton, G.M. Mace, R. Marten, S.S. Myers, S. Nishtar, S.A. Osofsky, S.K. Pattanayak, M.J. Pongsiri, C. Romanelli, A. Soucat, J. Vega, and D. Yach (2015), "Safeguarding human health in the Anthropocene epoch: report of The Rockefeller Foundation-Lancet Commission on planetary health," The Lancet, 386(10007), 1973-2028.

WHO (2007a), "WHO releases new guidance on insecticide-treated mosquito nets," World Health Organization, Geneva.

— (2007b), "Insecticide-treated mosquito nets: a WHO position statement," World Health Organization, Geneva.

(2010), "Constraints to Scaling Up the Health Millennium Development Goals: Costing and Financial Gap Analysis," World Health Organization, Geneva.

(2011), "Scaling up action against noncommunicable diseases: How much will it cost?," World Health Organization, Geneva.

(2015a), "WHO support to countries in accessing and utilizing resources from the Global Fund: A handbook," World Health Organization, Geneva.

(2015b), Guidelines for the Treatment of Malaria, 3rd ed., World Health Organization, Geneva.

(2016a), Density of physicians (total number per 1000 population) | Data, http://www.who.int/gho/health_workforce/physicians_density/en/, accessed December 8, 2016.

(2016b), GHO Road traffic deaths I Data, http://apps.who.int/gho/data/node.main.A997, accessed December 8, 2016. 
(2016c), Consolidated guidelines on the use of antiretroviral drugs for treating and preventing HIV infection: recommendations for a public health approach, World Health Organization.

(2016d), Global Health Observatory, Database, http://www.who.int/gho/en/, accessed December 7, 2016.

(2016e), Global Health Expenditure Database, Database, http://apps.who.int/nha/database, accessed December 7, 2016.

(2017a), GHO Obesity (body mass index $>=30$ ) (age-standardized estimate) | Data, http://apps.who.int/gho/data/view.main.CTRY2450A?lang=en, accessed January 4, 2017.

(2017b), Maternal mortality ratio (modeled estimate, per 100,000 live births) | Data, http://data.worldbank.org/indicator/SH.STA.MMRT, accessed January 4, 2017.

(2017c), Incidence of tuberculosis (per 100,000 people) | Data, http://data.worldbank.org/indicator/SH.TBS.INCD, accessed January 4, 2017.

(2017d), GHO Healthy life expectancy I Data, http://apps.who.int/gho/data/node.main.688, accessed January 4, 2017.

WHO, and UNICEF (2016a), Immunization Coverage I Data, //data.unicef.org/topic/child-health/immunization/, accessed December 8, 2016.

(2016b), WHO / UNICEF Joint Monitoring Programme: Data \& estimates, http://www.wssinfo.org/data-estimates/, accessed December 8, 2016.

Wiedmann, T.O., H. Schandl, M. Lenzen, D. Moran, S. Suh, J. West, and K. Kanemoto (2015), "The material footprint of nations," Proceedings of the National Academy of Sciences, 112(20), 6271-6276.

Williams, J.H., A. DeBenedictis, R. Ghanadan, A. Mahone, J. Moore, W.R. Morrow, and M. Torn (2012), "The technology path to deep greenhouse gas emissions cuts by 2050: the pivotal role of electricity," Science, 335(6064), 53-59.

Williams, J.H., B. Haley, F. Kahrl, A.D. Jones, M.S. Torn, and H. McJeon (2014), "Pathways to Deep Decarbonization in the United States," SDSN and IDDRI: New York and Paris.

Wiseman, A.W. (2013), "Policy responses to PISA in comparative perspective," PISA, Power, and Policy: the emergence of global educational governance, 303-322.

Wood, A. (2008), "Looking ahead optimally in allocating aid," World Development, 36(7), 1135-1151.

World Bank (1998), Assessing Aid: What Works, What Doesn't, and Why, Oxford University Press, Oxford.

_ (2010a), "The Cost to Developing Countries of Adapting to Climate Change. New Methods and Estimates," World Bank, Washington D.C.

(2010b), "World Development Report 2010," World Bank, Washington D.C.

- (2010c), "Economics of Adaptation to Climate Change: Synthesis Report," International Bank for Reconstruction and Development and World Bank, Washington, D.C.

(2011), "The Changing Wealth of Nations: Measuring Sustainable Development in the New Millennium," World Bank, Washington D.C.

(2012), "What a Waste: A Global Review of Solid Waste Management," Urban Development Seris - Knowledge Papers No. 15, World Bank, Washington D.C. (2013), "Global Development Horizons - Capital for the Future: Saving and Investment in an Interdependent World," World Bank, Washington, D.C.

(2016a), World Bank list of economies 2016, http://databank.worldbank.org/data/download/site-content/CLASS.xls, accessed December 7, 2016.

(2016b), World Development Indicators, http://data.worldbank.org/products/wdi, accessed December 7, 2016. 
(2016c), Poverty gap at $\$ 1.90$ a day (2011 PPP) (\%) | Data, http://data.worldbank.org/indicator/SI.POV.GAPS, accessed December 8, 2016.

(2016d), Logistics Performance Index (LPI), http://lpi.worldbank.org/international/global, accessed December 12, 2016.

(2016e), Worldwide Governance Indicators database, http://info.worldbank.org/governance/wgi/index.aspx\#home, accessed December 7, 2016.

(2017a), GINI index (World Bank estimate) | Data, http://data.worldbank.org/indicator/SI.POV.GINI, accessed January 4, 2017.

(2017b), Tax revenue (\% of GDP) | Data, http://data.worldbank.org/indicator/GC.TAX.TOTL.GD.ZS, accessed January 4, 2017.

— (2017c), GDP per capita, PPP (constant 2011 international \$) | Data, http://data.worldbank.org/indicator/NY.GDP.PCAP.PP.KD, accessed January 4, 2017.

World Data Lab (2017), World Poverty Clock, http://worldpoverty.io/, accessed June 10, 2017.

Yamey, G. (2004), "Roll Back Malaria: a failing global health campaign," BMJ : British Medical Journal, 328(7448), 1086-1087.

You, D., L. Hug, S. Ejdemyr, P. Idele, D. Hogan, C. Mathers, P. Gerland, J.R. New, L. Alkema, and United Nations Inter-agency Group for Child Mortality Estimation (2015), "Global, regional, and national levels and trends in under-5 mortality between 1990 and 2015, with scenario-based projections to 2030: a systematic analysis by the UN Inter-agency Group for Child Mortality Estimation," The Lancet, 386(10010), 2275-2286.

Younas, J. (2008), "Motivation for bilateral aid allocation: Altruism or trade benefits," European Journal of Political Economy, 24(3), 661-674.

Zelman, B., A. Kiszewski, C. Cotter, and J. Liu (2014), "Costs of eliminating malaria and the impact of the Global Fund in 34 countries," PloS One, 9(12), e115714.

Zhang, X., and E.A. Davidson (2016), "Sustainable Nitrogen Management Index (SNMI)," University of Maryland Center for Environmental Science, Maryland.

Zhang, X., E.A. Davidson, D.L. Mauzerall, T.D. Searchinger, P. Dumas, and Y. Shen (2015), "Managing nitrogen for sustainable development," Nature, 528(7580), $51-59$.

Zhao, J., M. Lama, E. Korenromp, P. Aylward, E. Shargie, S. Filler, R. Komatsu, and R. Atun (2012), "Adoption of Rapid Diagnostic Tests for the Diagnosis of Malaria, a Preliminary Analysis of the Global Fund Program Data, 2005 to 2010," PLoS One, 7(8), e43549. 
References 
On metrics and financing for the Sustainable Development Goals 
Summary 
The world has experienced unprecedented growth in average per capita incomes over the last 50 years, but many countries continue to face deep economic, social, and/or environmental challenges. These include persistent extreme poverty, poor outcomes in human health and education, widespread malnutrition, high inequality measured by income or other characteristics, poor access to infrastructure, growing water stress, the degradation of terrestrial and marine ecosystems, pollution, and climate change. Under business-as-usual trajectories the environmental challenges in particular are expected to worsen significantly. Enhanced international policy coordination and cooperation around shared goals is required to reverse these trends, and many developing countries require more external financial assistance.

In response governments have adopted international development goals, including the Millennium Development Goals (MDGs) and their successors, the Sustainable Development Goals (SDGs), which are to be achieved by 2030. These goals complement earlier tools for international policy coordination, notably the environmental conventions, such as the United Nations Framework Convention on Climate Change and the Convention on Biological Diversity.

This thesis contributes to the need to understand how progress towards the SDGs can be monitored, how investment needs for climate-resilient development and the SDGs can be estimated, and what lessons can be drawn for international financing mechanisms in support of the SDGs from the experience of the health sector under the MDGs. These issues represent important contemporary questions in the scientific and policy literature, as evidenced by the rapidly growing scientific literature on the SDGs to which this thesis contributes.

Chapter 2 introduces a novel SDG Index and Dashboards that combines official and science-based metrics to establish an SDG baseline for the 149 countries for which sufficient data are currently available. The SDG Index and Dashboards measure countries' distance from achieving the goals, assess overall performance, and identify implementation priorities for each country. We find that many countries' development models are imbalanced in favor of economic development and at the expense of social inclusion and environmental sustainability. We demonstrate the SDG Index' usefulness as an explanatory variable in studying policy objectives, such as subjective wellbeing and in identifying policy priorities. Moreover, the chapter identifies major data gaps for monitoring the SDGs and suggests ways in which these can be closed in coming years.

In Chapter 3 we consider the combined investment needs of development objectives in low-income country settings, as exemplified by the MDGs, and measures to adapt to a changing climate. Drawing on consensus investment needs for the MDGs in Africa, as established by the MDG Africa Steering Group, and the literature on investment needs for climate change adaptation, we propose and apply a methodology for integrating these assessments. The chapter reviews major line items in financing the MDGs and considers the nature and extent of additional measures to adapt to climate change, as well as associated financing needs. We find that climate change adaptation may increase total investment needs by some 40 percent. The analysis shows that development and adaptation measures need to be integrated along sectoral lines in order to facilitate implementation by governments.

Chapter 4 extends this analysis to propose an analytical framework for SDG needs assessments that translates the 17 SDGs into eight investment areas and introduces a preliminary score to assess the quality and suitability of needs assessment studies. Using this framework, published sector needs assessments are analyzed, harmonized, and consolidated to arrive at a first assessment of private and public investment needs 
for the SDGs in low- and lower-middle-income countries. Incremental spending needs in these countries are estimated at \$1.3-1.4 trillion per year. Approximately half of these incremental investments can be privately financed. Domestic resource mobilization can increase significantly, leaving an external financing gap of perhaps \$152-163 billion per year (equivalent to $0.22-0.26 \%$ of high-income countries' GDP) that must be met through international public finance, including Official Development Assistance. Globally, an incremental $1.5-2.5 \%$ of world GDP needs to be invested each year by the public and private sectors to achieve the SDGs in every country.

Turning to the financing of the SDGs, Chapter 5 investigates the experience of the Global Fund to Fight HIVIAIDS, Tuberculosis and Malaria in financing the rapid scaling up of proven health interventions observed since 2002. The chapter identifies 8 key design principles of the Global Fund that set the institution apart from other multilateral financing mechanisms. It then considers to what extent these design principles have enabled rapid progress in combating the three infectious diseases in a broad range of operating environments, including fragile countries and countries with poor governance. The chapter concludes that the Global Fund has performed better than expected at inception, and that the key design principles explain this success. Adopting these principles may help multilateral grant financing mechanisms focusing on other SDG priorities - such as education; access to energy, water, and sanitation; nutrition; and smallholder agriculture - improve the effectiveness of resource use and accelerate progress towards the goals.

In the Chapter 6, we investigate the Global Fund's Technical Review Panel (TRP) to determine whether it had succeeded in reconciling the competing needs of country ownership of development programs and the need to ensure effective use of scarce resources. We also investigate whether the demand-based application process generated funding allocations that were in line with the Global Fund's objective to direct funds towards the countries most in need. To answer these questions, we construct a novel dataset and conduct four sets of regression analysis using ordinary least squares and ordered logistic regression models. The chapter finds that the TRP operated in line with the Global Funds' objectives and allocated funding to countries most in need, though we find evidence that countries with large populations suppressed the volume of financing requested from the Global Fund. The evidence suggests that the TRP promoted learning on how to scale up disease control programs and that the Global Fund operated equally well across different country environments, including fragile and poorly governed countries. The chapter closes by considering the policy implications for financing the SDGs in health and other areas.

The concluding chapter summarizes the research findings and critically discusses the methodologies and data used in this thesis. It outlines suggestions for further research and summarizes policy implications for monitoring, implementing, and financing the SDGs. 
On metrics and financing for the Sustainable Development Goals 


\section{Acknowledgements}


I would like to thank my promoters Prof. Ekko van lerland and Prof. Pavel Kabat who accepted to supervise the thesis without knowing me well and in spite of my demanding day job at the United Nations Sustainable Development Solutions Network (SDSN). Ekko, you taught me how to frame rigorous research questions and how to push the analysis further than I often thought possible. Your comments on draft documents were always incisive and extremely helpful. I am particularly grateful for your guidance and flexibility in helping me adjust the focus of the thesis to new ideas and opportunities.

Pavel, I thank you for your trust in my ability to complete the PhD and for your encouragement to always aim higher. You have guided me in connecting my research to policy implications. You have a remarkable ability to inspire, and your advice on framing the overall research questions and the synthesis was particularly important.

I am deeply grateful and indebted to my co-promoter Prof. Jeffrey Sachs for mentorship, advice, and encouragement since 2002. Jeff, you have taught me that ideas and knowledge ultimately bend the arc of history. Your passion, erudition, humanity, and impatience in seeking faster progress have been an inspiration to me. They have changed my life. Throughout the thesis you have been a constant source of advice, guidance, and encouragement. Thank you for the many 7 am breakfast meetings to discuss ideas and review draft analyses for this thesis.

I would also like to thank my co-promoter Dr Jeroen Klomp for the careful review of the econometric analyses in this thesis and for your guidance on how to embed the research in the development economics literature. Your comments have always been spot on and important in navigating the peer review processes.

This thesis is intricately connected to my work at the SDSN and my earlier roles at the Millennium Project and UNDP. I have been very fortunate to work with many leading minds at the intersection of science and policy across the social and physical sciences. I am very grateful to the members of the SDSN Leadership Council, the national and regional SDSNs around the world, United Nations and government leaders aiming to steer this world into a safer direction, and the scientists making sense of it all. You have all given me the inspiration for the research covered in this thesis. Above all, I thank the wonderful colleagues in the SDSN Secretariat who have contributed in many ways to this research. You are recognized in the individual chapters.

A particular thank you goes out to the tremendous team of the Global Fund to Fight AIDS, Tuberculosis, and Malaria and your generosity in sharing data and patiently answering my questions. You embody the best of multilateral cooperation: Dedicated, sharp, never afraid to hear feedback even when it is negative, and always keen to make the Global Fund more effective.

I am grateful for Wil den Hartog and Anneke Geselschap for your wonderful support in arranging this thesis and for making the defense possible.

I am grateful for all my friends who have kept me firmly grounded during the thesis. Josh, you have been an example and a tremendous help throughout. I wished there had been more time to discuss ideas with Thomas.

Above all, I must thank my family! Shivani, you have been unwavering in your support and encouragement in spite of the many late nights and early mornings that were taken up by the thesis. Your love and support mean everything to me. Annika and Leo, I love discussing this world with you. I thank you for your boundless curiosity and for always knowing the right moment when to shut down my computer. Sigrun and Henner, you have taught me the importance of scholarship, the joy of learning about new fields, and 
Acknowledgements

the importance of modesty. Your support has been invaluable to me, and I am proud to walk in your footsteps. 
On metrics and financing for the Sustainable Development Goals 
Biography 
Guido Schmidt-Traub was born on 16 May 1972 in Berlin, Germany. He grew up in Germany and attended the United World College of the Atlantic in Wales, graduating in 1991.

Guido studied physical chemistry at the Albert-Ludwigs Universität Freiburg (Germany), the Université Paul Sabatier Toulouse (France), and the Freie Universität Berlin (Germany) where he obtained an M.Sc. ('Diplom') in physical chemistry in 1998. His Masters thesis examining mechanisms of tropospheric smog formation. He studied Economics at Université des Sciences Sociales Toulouse and Freie Universität Berlin. From 1998-2000 he attended Oxford University (St Antony's College) as a Rhodes Scholar and graduated with an M.Phil. in Economics. His thesis investigated the impact of climate change policies on industrial competitiveness across the European Union.

Following graduation from Oxford, Guido worked briefly for McKinsey \& Co. in Germany before joining IndexIT Scandinavia, an advisor on technology commercialization to universities and technology companies in Scandinavia and the UK. He also ran Banyan S.A., a private equity investment fund focusing on early-stage technology companies.

In 2002 Guido joined the UN Millennium Project commissioned by UN Secretary-General Kofi Annan and directed by Prof. Jeffrey Sachs. Based at the United Nations in New York, the Millennium Project was tasked with developing an implementation plan for achieving the Millennium Development Goals (MDG). Guido facilitated the task forces on water and sanitation, urban development, ecosystems, and science \& technology. He led the Millennium Project's work on MDG needs assessments and became Associate Director in 2005. In 2006 the team was integrated into UNDP. Under Guido's leadership, the team advised over 70 governments in Africa, Latin America, and Asia on aligning their national development strategies with the SDGs. He also helped establish the MDG Africa Steering Group chaired by UN Secretary-General Ban Ki-moon and led its technical work.

In 2008, Guido became Director at South Pole Carbon Asset Management in Zurich (Switzerland), where he was responsible for developing programmatic approaches to reducing greenhouse gas emissions and advisory services. Since 2009 he served as climate change advisor to the Africa Progress Panel chaired by Kofi Annan. In this capacity, he published on climate change and carbon markets in Africa. In 2010, Guido was appointed founding CEO of CDC Climat Asset Management in Paris. He built up an investment company in low-carbon energy projects that was regulated by the French financial markets regulator.

Since 2012 Guido serves as Executive Director of the UN Sustainable Development Solutions Network (SDSN), which is directed by Jeffrey Sachs with offices in New York and Paris. The SDSN operates under the auspices of the UN Secretary-General to support the design and implementation of the Sustainable Development Goals (SDGs) and the Paris Climate Agreement. With partner organizations, the SDSN has launched the Deep Decarbonization Pathways Project to develop national pathways for decarbonizing energy systems; the World in 2050 to develop integrated global and regional pathways for achieving the SDGs; and the Food, Agriculture, Biodiversity, Land-use, and Energy (FABLE) initiative to develop integrated national and global pathways towards sustainable land-use. The SDSN has played a central role in the design of the SDGs, and its Deep Decarbonization Pathway Project has contributed to a successful COP21 and the inclusion of long-term low-emission development strategies (Article 4.19 ) in the Paris Climate Agreement. At the SDSN Guido has published inter alia on SDG needs assessments and financing, long-term pathways for the SDGs, the SDG Index and Dashboards, and the design of the SDGs. 
Guido has taught graduate courses at Columbia University (New York, United States), Science Politiques and Université Dauphine (Paris, France). He is a member of the Governing Council of Future Earth, an advisor to the Business and Sustainable Development Commission, a member of the Urban Leadership Council, and a member of the Advisory Council of Atlantic College.

Since 2015, Guido combined his position at the SDSN with an external PhD at the Environmental Economics and Natural Resources Group at Wageningen University. In this PhD he explored baselines for the SDGs, integrating climate change adaptation into development needs assessments, investment needs for the SDGs, and lessons from the Global Fund to Fight HIVIAIDS, Tuberculosis and Malaria for financing the SDGs.

He resides in Paris with his wife and two children. 
On metrics and financing for the Sustainable Development Goals 
Selected publications 


\section{Journal articles}

Schmidt-Traub, G. forthcoming. "The role of the Technical Review Panel of the Global Fund to Fight HIVIAIDS, Tuberculosis and Malaria: An analysis of grant recommendations," Health Policy and Planning.

Obersteiner, M., Bednar, J., Wagner, F., Gasser, T., Ciais, P., Forsell, N., Frank, S., Havlík, P., Valin, H., Janssens, I., Penuelas, J., Schmidt-Traub, G. forthcoming. "An options assessment on how to spend a dwindling GHG budget." Nature Climate Change.

Schmidt-Traub, G., Kroll, C., Teksoz, K., Durand-Delacre, D., Sachs, J.D. 2017. "National baselines for the Sustainable Development Goals assessed in the SDG Index and Dashboards." Nature Geoscience. 10: 547-55.

Sachs, J.D., Schmidt-Traub, G. 2017. "Global Fund Lessons for the Sustainable Development Goals." Science. 6333: 32-33.

Sachs, J.D., Schmidt-Traub, G., Williams, J. 2016. "Pathways to Zero Emissions. Nature Geoscience." 9: 799-801.

Schmidt-Traub, G. 2016. "Wege zu Null Emissionen." Weltsichten. November: 40-43.

Schmidt-Traub, G. 2015. "Drei Stellschrauben sollt Ihr sein. Die Realisierung der Nachhaltigkeitsziele." Politische Ökologie. 2015 (143): 76-81.

Fankhauser, S., Schmidt-Traub, G. 2011. "From adaptation to climate-resilient development. The costs of climate-proofing the Millennium Development Goals in Africa." Climate and Development, 3(2): 94-113.

Schmidt-Traub, G. 2009. "The Millennium Development Goals and human rightsbased approaches: moving towards a shared approach." The International Journal of Human Rights, 13(1): 72-8.

Melnick, D.J., McNeely, J., Kakabadse-Navarro, Y., Schmidt-Traub, G., Sears, R. 2005. "The Millennium Project: the positive health implications of improved environmental sustainability." The Lancet. 365: 723-25.

McArthur, J.W., Sachs, J.D., Schmidt-Traub, G. 2005. "Response to Amir Attaran." PLoS Medicine. 2(11): 1190-1191.

McArthur, J.W., Schmidt-Traub, G. 2005. "Bringing 2015 to 2005: Implementing the Millennium Development Goals today." International Aid \& Trade. August: 1-5.

Sachs, J.D., McArthur, J.W., Schmidt-Traub, G. 2005. "A European Development Fund For The MDGs." Revamping Development Efforts: An Assessment of Development Policy in the EU, European Policy Centre Issue Paper 36. July: 21-24.

Sachs, J.D., McArthur, J.W., Schmidt-Traub, G. 2005. "What it will take to meet the Millennium Development Goals." Sustainable Development International. Fifteenth Edition, September: 13-19.

Sachs, J.D., McArthur, J.W., Schmidt-Traub, G., Kruk, M., Bahadur, C., Faye, M., McCord, G. 2004. "Ending Africa's Poverty Trap." Brookings Papers on Economic Activity 2004(1): 117-240.

\section{Working papers}

Schmidt-Traub, G. 2017. "A Long-Term Transformation Pathways Initiative (LTTPI) for the G20." G20 Insights. Institute for the World Economy. Kiel.

Sachs, J.D., Schmidt-Traub, G., Williams, J., Segafredo, L., Colombier, M., Ribera, T., Waisman, H. 2015. "Why Climate Policy needs long-term Deep Decarbonization Pathways." SDSN Working Paper. Sustainable Development Solutions Network. Paris and New York.

Schmidt-Traub, G. 2015. "Investment Needs to Achieve the Sustainable Development Goals: Understanding the Billions and Trillions." SDSN Working Paper. Sustainable Development Solutions Network. Paris and New York.

Schmidt-Traub, G., Sachs, J.D. 2015. "Financing Sustainable Development: Implementing the SDGs through Effective Investment Strategies and Partnerships." 
SDSN Working Paper. Sustainable Development Solutions Network. Paris and New York.

Sustainable Development Solutions Network. 2015. "Key Elements of a Successful Addis Ababa Accord on Financing for Sustainable Development." Sustainable Development Solutions Network. Paris and New York. [Lead Writer]

Sustainable Development Solutions Network. 2015. "Key Elements for Success on Climate Change Mitigation at COP21 in Paris." Sustainable Development Solutions Network. Paris and New York. [Lead Writer]

Sustainable Development Solutions Network Secretariat 2014. "Framing Sustainable Development Goals, Targets, and Indicators." Sustainable Development Solutions Network. Paris and New York. [Lead Writer]

Rockström, J., Sachs, J.D., Öhman, M.C., Schmidt-Traub, G. 2013. "Sustainable Development and Planetary Boundaries." Background paper for the High-Level Panel of Eminent Persons on the Post-2015 Development Agenda. Sustainable Development Solutions Network. Paris and New York.

Sachs, J.D., Schmidt-Traub, G. 2013. "Financing for development and climate change post-2015." Background paper for the High-Level Panel of Eminent Persons on the Post-2015 Development Agenda. Sustainable Development Solutions Network. Paris and New York.

Sachs, J.D., Schmidt-Traub, G. 2013. "The Structural Transformations for Sustainable Development." Background paper for the High-Level Panel of Eminent Persons on the Post-2015 Development Agenda. Sustainable Development Solutions Network. Paris and New York.

Schmidt-Traub, G. 2011. "Transforming Africa's Development Path. What Durban needs to Deliver." Policy Brief Africa Progress Panel. Geneva.

Schmidt-Traub, G. 2010. "Finance for climate-resilient development in Africa. An agenda for action following the Copenhagen Conference." Working Paper Africa Progress Panel.

Schmidt-Traub, G. 2010. Mobilizing carbon finance for development in Africa. Opportunities for action for German development cooperation. KfW. Frankfurt.

Schmidt-Traub, G., Wylie B. 2009. "Kick-starting Africa's carbon markets. The potential for programmatic CDM." Africa Progress Panel. Geneva.

Schmidt-Traub, G. 2009. "Climate Change: A Call to Action for African Leaders." Discussion Paper issued by Africa Progress Panel, African Development Bank, United Nations Economic Commission for Africa, United Nations Environment Programme. Geneva.

Sachs, J.D., Schmidt-Traub, G. 2006. "Hitting the Target. Words into Action." IMF and World Bank Annual Meetings, September 2006. Washington D.C.

Sachs, J.D., Schmidt-Traub, G. 2006. "The Year of Implementation for the MDGs." In Commonwealth Finance Minsters Reference Report 2006. Commonwealth Secretariat. 2006. Henley Media Group Ltd. London. UK, pp. 96-98.

Sachs, J.D., McArthur, J.W., Schmidt-Traub, G., Bahadur, C., Faye, M., Kruk, M. 2004. "Millennium Development Goals Needs Assessments. Country Case Studies of Bangladesh, Cambodia, Ghana, Tanzania and Uganda." UN Millennium Project Working Paper. UN Millennium Project. New York.

Sachs, J.D., McArthur, J.W., Schmidt-Traub, G., Kruk, M., Bahadur, C., Faye, M., McCord, G. 2004. "Millennium Development Goals Needs Assessments for Ghana, Tanzania, and Uganda. Background Paper to "Ending Africa's Poverty Trap." Brookings Papers on Economic Activity 1:2004. UN Millennium Project Working Paper. UN Millennium Project. New York. 


\section{Reports}

Prakash, M., Teksoz, K., Espey, J., Sachs, J.D., Shank, M., Schmidt-Traub, G. 2017. "Achieving a Sustainable Urban America: SDSN's first U.S. Cities SDG Index." Sustainable Development Solutions Network. Paris and New York.

Sachs, J.D., Schmidt-Traub, G., Kroll, C., Teksoz, K., Durand-Delacre, D. 2017. "SDG Index and Dashboards Report 2017. Global Responsibilities." Bertelsmann Stiftung and Sustainable Development Solutions Network. Gütersloh and New York. [Lead Writer]

Sachs, J.D., Schmidt-Traub, G., Kroll, C., Teksoz, K., Durand-Delacre, D. 2016. "SDG Index and Dashboards Report 2016." Bertelsmann Stiftung and Sustainable Development Solutions Network. Gütersloh and New York. [Lead Writer]

Sustainable Development Solutions Network. 2015. "Indicators for Sustainable Development Goals - Draft for Public Consultation." Sustainable Development Solutions Network. Paris and New York. [Lead Writer]

Sustainable Development Solutions Network. 2013. "An Action Agenda for Sustainable Development. A Report to the UN Secretary-General." Sustainable Development Solutions Network. Paris and New York. [Lead Writer]

Africa Progress Panel. 2012. "Africa Progress Report 2012 - Jobs, Justice and Equity: Seizing Opportunities in Times of Global Change." Africa Progress Panel. Geneva. [Contributing Author]

Beaurain, F., Schmidt-Traub, G. 2010. "Developing CDM Programmes of Activities: A Guidebook." South Pole Carbon Asset Management. Zurich.

MDG Africa Steering Group. 2008. "Achieving the Millennium Development Goals in Africa." United Nations. New York. [Lead Writer]

United Nations Environment Program. 2007. "Global Environment Outlook 4." UNEP. Nairobi. [Contributing Author]

UN Millennium Project. 2006. "Preparing National Strategies to Achieve the MDGs: A Handbook." UN Millennium Project. New York. [Lead author with Chandrika Bahadur and Margaret Kruk]

UN Millennium Project. 2005. "Investing in Development: A Practical Plan to Achieve the Millennium Development Goals. Overview." Final Overview Report of UN Millennium Project Submitted to the UN Secretary-General. Earthscan. New York. [Co-author.]

UN Millennium Project. 2005. "Investing in Development: A Practical Plan to Achieve the Millennium Development Goals." Final Full Report of UN Millennium Project. Earthscan. New York. [Co-author]

UN Millennium Project. 2005. "Environment and Human Well-Being. A Practical Strategy." UN Millennium Project. Earthscan. New York. [Lead author with Melnick, D.J., J. McNeely, Y. Kakabadse-Navarro, and R. Sears.]

UNDP. 2003. "Human Development Report 2003: The Millennium Development Goals - A Compact Among Nations to End Human Poverty." Oxford University Press. New York. [Contributing author.]

\section{Book chapters}

Schmidt-Traub, G., Durand-Delacre, D., Teksoz, K. 2016. "The SDG Dashboard and Index: Getting Started with the Sustainable Development Goals." In Better Policies for Sustainable Development 2016. A New Framework for Policy Coherence. OECD. Paris.

Schmidt-Traub, G. 2016. "Funding the Sustainable Development Goals." In SDGs: The People's Agenda. United Nations Association UK. London.

Schmidt-Traub, G. 2012. "Managing the risk of climate change and climate change regulation." In Clean Energy Investing. Private Equity International \& Capital Dynamics. London. 
Schmidt-Traub, G. 2011. "Climate finance - opportunities for project co-financing." In Africa Energy Yearbook 2011. EnergyNet Ltd. London.

Jobarteh, D., Schmidt-Traub, G. 2010. "Improving regional representation: Practical measures to take the carbon markets to Least Developed Countries." In Greenhouse Gas Market 2010. International Emissions Trading Association. Washington D.C.

Schmidt-Traub, G. 2007. "Meeting the Millennium Development Goals and the role for ICT." In ICT for Education and Development. (edited by Carlos Rodriguez). United Nations. Geneva.

Schmidt-Traub, G., Cho, A. 2006 "From Assessment to Action. Operationalizing Environmental Sustainability at the National Level." In Review of Millennium Ecosystem Assessment for World Resources Institute. World Resources Institute. Washington, D.C.

Wall, D., Rabbinge, R., Gallopin, G., Khoday, K., Lewis, N., Lubchenco, J., Melillo, J., Schmidt-Traub, G., Sombilla. M. 2005 "Implications for Achieving the Millennium Development Goals." In Ecosystems and Human Well-Being. Volume 3. Millennium Ecosystem Assessment, Island Press. Washington D.C. pp. 549584.

McArthur, J.W., Sachs, J.D., Schmidt-Traub, G. 2005. "Le projet du Millénaire pour les OMD: objectifs et approche méthodologique au plan national." In L'Afrique et les Objectifs du Millénaire pour le Développement. (edited by A. Rhazaoui, LJ. Grégoire, and S. Mellali). Economica. Paris. pp. 487-514. 
On metrics and financing for the Sustainable Development Goals 
Funding 
On metrics and financing for the Sustainable Development Goals

Part of the research described in this thesis was funded by the Swedish International Development Cooperation Agency (Sida), the Bertelsmann Stiftung, and the Sustainable Development Solutions Network (SDSN). 
Funding 
On metrics and financing for the Sustainable Development Goals 
Photo credits 
On metrics and financing for the Sustainable Development Goals

Cover photo: Sustainable Development Goals logos, United Nations

Chapter 1 (page 11): Fields outside Lilongwe, Malawi. 2005. Guido Schmidt-Traub Chapter 2 (page 27): Adapted with permission from Sachs et al. (2017)

Chapter 3 (page 77): Farmers. Northern Rwanda. 2004. Guido Schmidt-Traub

Chapter 4 (page 97): Sustainable Development Goals logos, United Nations

Chapter 5 (page 125): Malaria patient. Tiby, Mali. 2008. Guido Schmidt-Traub

Chapter 6 (page 137): Health clinic. Segou, Senegal. 2008. Guido Schmidt-Traub

Chapter 7 (page 165): Fez, Morocco. 2008. Guido Schmidt-Traub 


\section{Photo credits}


